\title{
Analysis of two kinetically distinct components of transmitter release at a fast synapse of the mammalian central nervous system
}

\author{
Dissertation \\ zur Erlangung des Doktorgrades \\ der Mathematisch-Naturwissenschaftlichen Fakultäten \\ der Georg-August-Universität zu Göttingen
}

vorgelegt von

Markus Wölfel

aus Göttingen

Göttingen 2004 
D 7

Referent:

Prof. Dr. Erwin Neher

Korreferent:

Prof. Dr. Andreas Stumpner

Tag der mündlichen Prüfung:

27.01.2005 


\section{To my dear parents}





\section{Table of Contents}

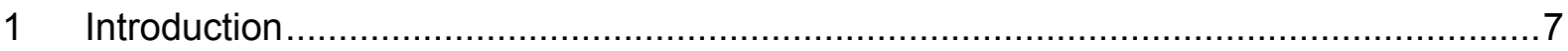

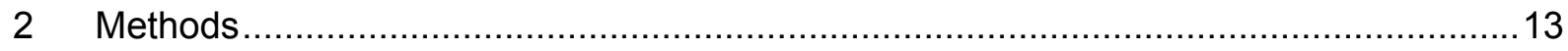

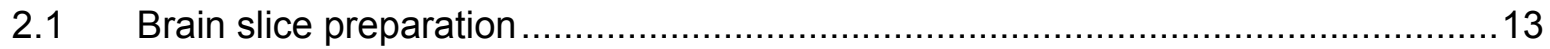

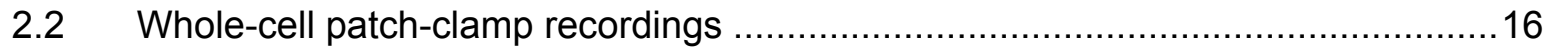

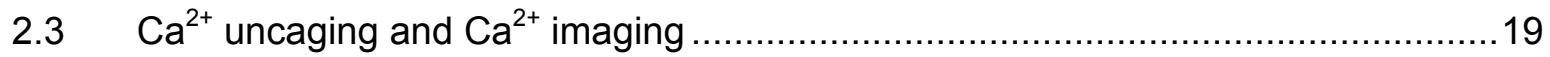

2.3.1 Calibration of the Calcium concentration measurements ............................23

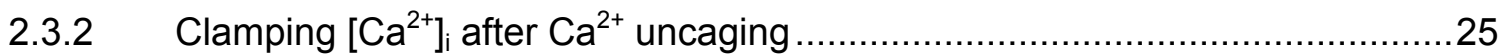

2.3.3 Spatial $\left[\mathrm{Ca}^{2+}\right]_{\mathrm{i}}$ homogeneity after $\mathrm{Ca}^{2+}$ uncaging......................................29

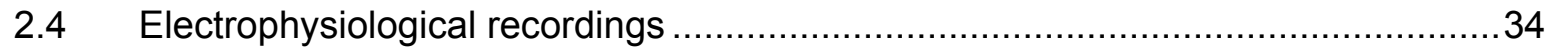

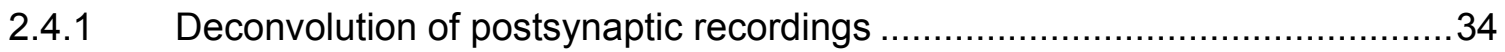

2.4.2 Fitting the cumulative release time courses................................................ 36

2.4.3 Presynaptic membrane capacitance measurements ...................................43

2.4.4 Large postsynaptic EPSCs leading to amplifier non-linearities ......................43

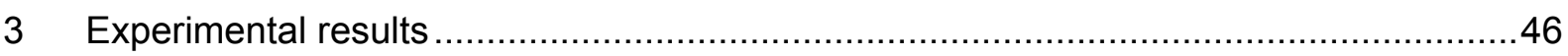

3.1 Depolarization induced release components.................................................46

3.1.1 Two components of release during AP-like stimuli .......................................46

3.1.2 Comparing deconvolution results to $C_{m}$ estimates....................................49

3.2 Two release components during $\mathrm{Ca}^{2+}$ uncaging .............................................53

3.3 Selective depletion of the FRP by short presynaptic depolarizations ....................61

3.3.1 Pre-depletion of the FRP followed by $\mathrm{Ca}^{2+}$ uncaging .................................61

3.3.2 Pre-depletion of the FRP in double-depolarization experiments ....................69

3.4 Submaximal release from the fast release component...................................... 75

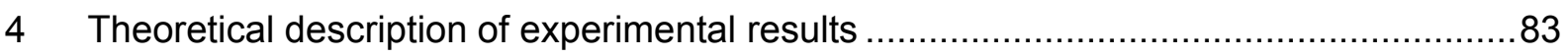

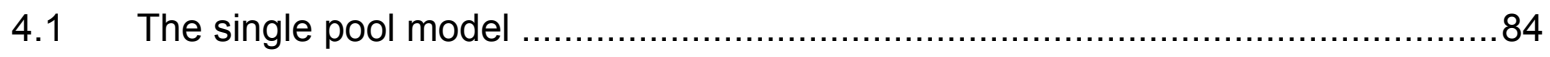

4.2 A fast $\left[\mathrm{Ca}^{2+}\right]_{\mathrm{i}}$ decay cannot account for the appearance of the

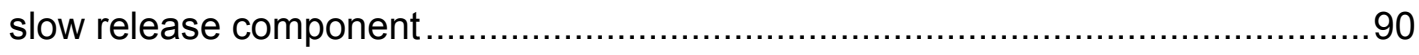

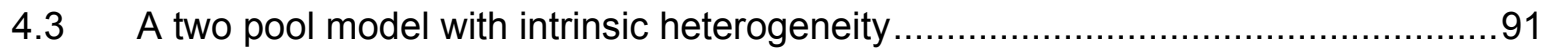

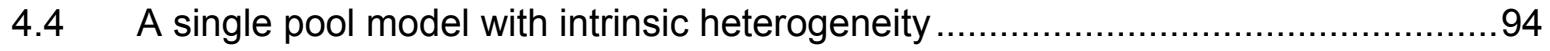

4.5 Analysis methods also prove for simulated release ...........................................98

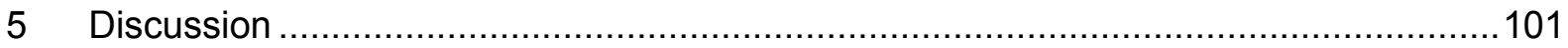

5.1 Two apparent release components based on intrinsic heterogeneity.................102

$5.2 \quad$ Intrinsic heterogeneity within a homogeneous pool of vesicles .........................107

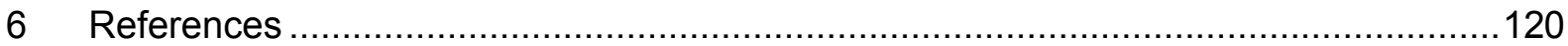




\section{Summary}

Two kinetically distinct components of transmitter release have been observed in $\mathrm{Ca}^{2+}$ uncaging experiments at a CNS synapse in rats, and both release components showed a steep dependence of release kinetics on $\left[\mathrm{Ca}^{2+}\right]_{i}$.

These findings were based on simultaneous pre- and postsynaptic whole-cell voltage-clamp recordings from the calyx of Held synapse, combined with presynaptic $\mathrm{Ca}^{2+}$ uncaging and $\left[\mathrm{Ca}^{2+}\right]_{\mathrm{i}}$ measurements. Presynaptic transmitter release rates were deconvolved from postsynaptic excitatory currents, which allowed for a correlation between obtained release kinetics and stimulus intensities in terms of presynaptic $\left[\mathrm{Ca}^{2+}\right]_{\text {. }}$. Furthermore, results could be confirmed by measurements of presynaptic changes in $\mathrm{C}_{\mathrm{m}}$.

In control experiments, possible artifacts that might have produced apparent biphasic release during $\mathrm{Ca}^{2+}$ uncaging stimuli were examined. $\mathrm{Ca}^{2+}$ uncaging led to a step-like and spatially homogeneous elevation of $\left[\mathrm{Ca}^{2+}\right]_{i}$, and moreover, it was shown that AMPA-receptor saturation, or desensitization were unlikely to have marked effects. Also the impact of rapid refilling of the readily-releasable pool of vesicles was investigated. It was found, that refilling could not account for release to become biphasic, especially regarding the steep $\left[\mathrm{Ca}^{2+}\right]_{\mathrm{i}}$ dependence of the slow release component. Therefore, intrinsic heterogeneity in release probabilities was proposed as the mechanism underlying biphasic release kinetics after $\mathrm{Ca}^{2+}$ uncaging. But still, an additional positional heterogeneity within the fast release component was found to affect fast release during presynaptic depolarizations.

Furthermore, the relative contribution of the fast and the slow release component to total release was found to depend on $\left[\mathrm{Ca}^{2+}\right]_{\mathrm{i}}$. At higher $\left[\mathrm{Ca}^{2+}\right]_{\mathrm{i}}$, more vesicles with fast kinetics were released as compared to milder $\mathrm{Ca}^{2+}$ uncaging stimuli. Thus, submaximal release of the fast release component at low $\left[\mathrm{Ca}^{2+}\right]_{i}$ was observed, for the first time at a CNS synapse, and this behavior cannot be explained by previous release models for the calyx of Held synapse.

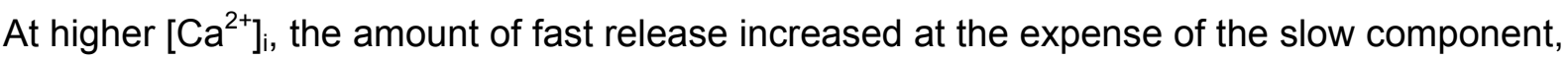
which indicates convertibility between fast and slowly releasing vesicles, and moreover, slowing of the release time constant of the fast component after modest pre-stimulation was observed experimentally.

Based on these findings, a single pool of readily-releasable vesicles with intrinsic heterogeneity was suggested as the mechanism underlying the observed phenomena. Simulations of release confirmed the ability of this novel release model to predict not only biphasic release, but also submaximal fast release at low $\left[\mathrm{Ca}^{2+}\right]_{\mathrm{i}}$, as experimentally observed. 


\section{Introduction}

Animal and human behavior is generated by neurons and neural network function. To understand how a specific behavior is implemented in a corresponding cluster of neurons, the interaction between neurons within this cluster is of basic interest. This interaction is not static, but dynamically regulated, depending on other neurons, neural networks, the state of the animal, and the environment. Processing and modulation of information mainly takes place at connections between two neurons, the synapses, where signals arrive that were propagated electrically within a neuron, often in form of an action potential (AP). An AP is converted into a chemical signal at the presynaptic terminal, when presynaptic membrane depolarizations induce the following sequence of events (Kandel et al, 2000; Zigmond et al., 1999). First, voltage gated Calcium $\left(\mathrm{Ca}^{2+}\right)$ channels open at elevated membrane potentials, and as a consequence, $\mathrm{Ca}^{2+}$ flows into the cell due to a large concentration gradient with a low intracellular $\mathrm{Ca}^{2+}$ concentration $\left(\left[\mathrm{Ca}^{2+}\right]_{\mathrm{i}}\right)$. After entering the cell, $\mathrm{Ca}^{2+}$ binds to $\mathrm{Ca}^{2+}$ sensors attached to synaptic vesicles. This in turn induces a lowering of the energy barrier for vesicles to fuse with the cell membrane, and therefore, the probability of vesicle fusion is increased markedly. When fusion occurs, neurotransmitters stored within the vesicles are released into the extracellular space, and then diffuse across the synaptic cleft to the postsynaptic neuron. Having arrived at the postsynaptic cell, the neurotransmitter molecules bind to specific receptors, and thus exert certain effects on the postsynaptic neuron. In the case of excitatory ionotropic neurotransmitters, receptor molecules will open pores which allow for cation flux into the cell, so that the postsynaptic membrane potential will increase. In this way, the chemical signal is again converted into an electrical signal, and the AP, generated in the postsynaptic cell by temporal and spatial summation, can travel through the neuron to finally arrive at the next synaptic connection. The most abundant excitatory neurotransmitter in the central nervous system (CNS) of mammals is (L-)glutamate, and the major known ionotropic glutamate receptors are of AMPA-, Kainate, and NMDA-type.

\section{The calyx of Held synapse}

Several important and detailed insights into synaptic function could be achieved at large model synapses. At the frog neuromuscular junction, it was shown first that neurotransmitter release occurs in discrete quanta (Katz, 1969), which already indicated neurotransmitter release from packages of specific size such as vesicles. At another large synapse, the giant squid stellate ganglion, a direct relation of presynaptic $\mathrm{Ca}^{2+}$ currents and postsynaptic 
membrane potentials could be identified (Llinas et al., 1981), another groundbreaking discovery in the field of neuroscience.

More recently, a large glutamatergic synapse of the mammalian CNS, the calyx of Held, has become available for electrophysiological studies (Held, 1893; Forsythe, 1994; Borst et al., 1995; Schneggenburger et al., 1999; Fig. 1). This synapse is formed in the auditory pathway in the brainstem (Fig. 1A) of most mammalian species, and is important for sound source localization (Oertel, 1997; Trussel, 1997). After sound perception in the cochlea, the calyx of Held is the third synaptic connection in a pathway for contralateral inhibition, which presumably leads to intensity comparison of sound arriving at either ear (Forsythe et al., 1995; Fig. 1A). The differences in sound intensity from each ear can be used to identify the sound source in aural space. For this task, the calyx of Held has developed to a highly reliable synapse, in the sense that every AP arriving at the presynaptic terminal will lead to a postsynaptic AP with a high probability (Borst et al., 1995; Schneggenburger et al., 1999; Taschenberger and von Gersdorff, 2000), and with fast kinetics (Schneggenburger and Neher, 2000; Bollmann et al., 2000). But not only the reliability of synaptic transmission makes this model synapse interesting for neuroscientific approaches. Each postsynaptic neuron is contacted by only one presynaptic calyx of Held terminal (Forsythe, 1994), so that polysynaptic information integration in the postsynaptic neuron does not play a significant role. Moreover, the presynaptic terminal itself is of exceptionally large size, which allows to perform whole-cell voltage-clamp recordings from the presynaptic site (Forsythe, 1994; Borst et al., 1995). Since both the presynaptic terminal and the contacted postsynaptic soma, are accessible to whole-cell recordings, many technical approaches can be utilized to study synaptic transmission in great detail.

To unveil properties of synaptic transmission, electrophysiological, optical, pharmacological and molecular techniques (Wimmer et al., 2004) were applied at the calyx of Held synapse, including presynaptic $\mathrm{Ca}^{2+}$ current recordings (Borst et al., 1995; Borst and Sakmann, 1996), presynaptic $\left[\mathrm{Ca}^{2+}\right]_{i}$ measurements (Helmchen et al., 1997), and presynaptic $\mathrm{Ca}^{2+}$ uncaging (Schneggenburger et al., 1999; Schneggenburger and Neher, 2000; Bollmann et al., 2000). Especially a fast component of presynaptic transmitter release was elaborately described, and found to determine information transmission at this synapse (Schneggenburger and Neher, 2000; Bollmann et al., 2000; Sakaba and Neher, 2001b). The $\mathrm{Ca}^{2+}$ sensor underlying this fast component of transmitter-release was estimated to bind up to five $\mathrm{Ca}^{2+}$ ions before triggering vesicle fusion (Schneggenburger and Neher, 2000; Bollmann et al., 2000). This agrees well with findings from molecular studies on possible $\mathrm{Ca}^{2+}$ sensors. The most likely molecular $\mathrm{Ca}^{2+}$ sensor triggering fusion is Synaptotagmin 1 (Brose et al., 1992; Chapman 

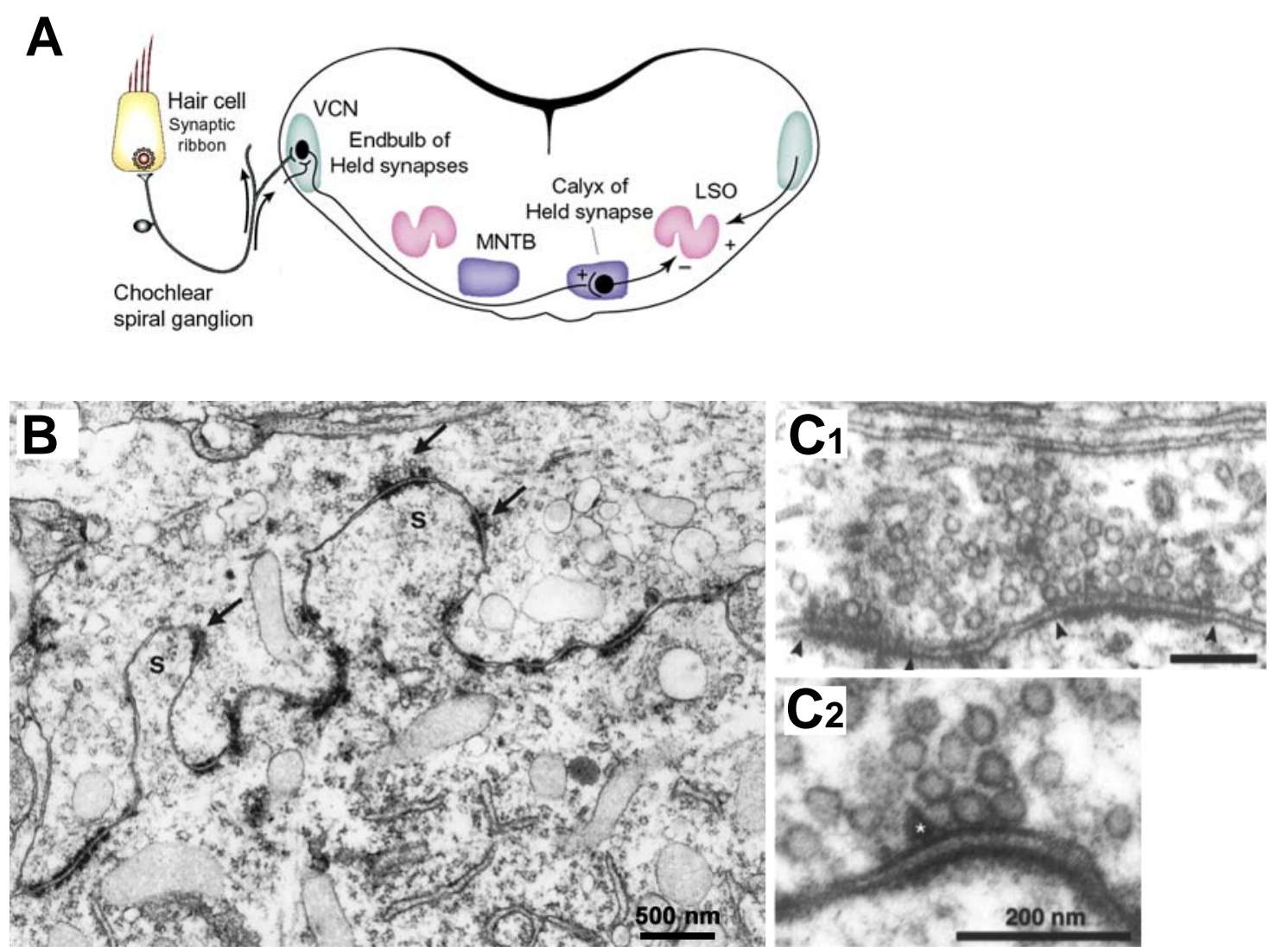

Fig. 1 The calyx of Held: position in the brainstem, and ultrastructure of the synaptic contact

A Illustration of a brainstem slice including the MNTB, where calyces of Held are located. Auditory information from inner hair cells in the cochlea passes through the spiral ganglion to the endbulbs of Held in the VCN. From there, globular bushy cells form calyx of Held synapses on principal neuron somata in the contralateral MNTB, finally leading to contralateral inhibition in the LSO. B - C Electron microscopic images of calyces of Held and the postsynaptic soma. A The opposed pre- and postsynaptic membranes (electron-dense bilayer band) are partially girded by presynaptic active zones together with postsynaptic densities (short stretches of dark, granular material, see arrows). Presynaptic vesicles accumulate at active zones (arrows). Image taken from Sätzler et al. (2002; rat P9; "s": spiny protrusion). C Magnified view of synaptic contacts (taken from Taschenberger et al., 2002; C1: P5, C2: P14; both scales equal $200 \mathrm{~nm}$ ). Postsynaptic densities (limits marked by arrowheads) are opposed by accumulations of vesicles at presynaptic active zones.

and Jahn, 1994; Davis et al., 1999; Fernández-Chacón et al., 2001; Sugita et al., 2002; Sørensen et al., 2003; for reviews, see Koh and Bellen, 2003; Yoshihara et al., 2003), which has $5 \mathrm{Ca}^{2+}$ binding sites at two $\mathrm{C}_{2}$ domains. The $\mathrm{C}_{2} \mathrm{~A}$ domain binds $3 \mathrm{Ca}^{2+}$ ions, and $\mathrm{C}_{2} \mathrm{~B}$ domain binds another $2 \mathrm{Ca}^{2+}$ ions. This consistence of biophysical and biochemical findings further speak for Synaptotagmin 1 being the $\mathrm{Ca}^{2+}$ sensor. However, at the calyx of Held 
synapse, the isoform Synaptotagmin 2, not Synaptotagmin 1 is expressed (Wölfel and Schneggenburger, unpublished), but Synaptotagmin 2 has very similar biochemical properties as compared to Synaptotagmin 1 (Geppert et al., 1991; Sugita et al., 2002). Thus, at the calyx of Held, Synaptotagmin 2 is likely to be the $\mathrm{Ca}^{2+}$ sensor triggering vesicle fusion.

\section{Two components of release with distinct release probabilities}

The events of information transmission at synaptic contacts have already been studied in great detail, but still, they are far from being completely understood. At several CNS synapses, transmitter release was shown to occur with two kinetically distinct components (Goda and Stevens, 1994; Hsu and Jackson, 1996; Mennerick and Matthews, 1996; Murthy et al., 1997; Sakaba et al., 1997; von Gersdorff et al., 1998; Yawo, 1999; Sakaba and Neher, 2001a; Sakaba and Neher 2001b). But also at neuromuscular junctions (Rahamimoff and Yaari, 1973), and in chromaffin cells (Neher and Zucker, 1993; Horrigan and Bookman, 1994; Seward and Nowycky, 1996; Moser and Neher, 1997; Voets et al., 1999; Voets, 2000) biphasic behavior of transmitter release could be observed. While the fast, highly synchronous release component has already been described in detail, origin and properties of the slow release component are still unclear.

The major hypotheses for mechanisms underlying heterogeneous release probabilities (Walmsley et al., 1988; Neher and Zucker, 1993; Hsu and Jackson, 1996 ; Neher, 1998; Xu et al., 1998; Voets et al., 1999; Xu et al, 1999; Voets, 2000; Sakaba and Neher, 2001a) separate into two classes. The first class is based on positional heterogeneity (Fig. 2, left panels), where different distances between the source of the $\mathrm{Ca}^{2+}$ signal, i.e. between voltage activated $\mathrm{Ca}^{2+}$ channels and the $\mathrm{Ca}^{2+}$ sensors located at vesicles, leads to inhomogeneous release probabilities for different vesicles (Neher and Zucker, 1993; Neher, 1998). When voltage activated $\mathrm{Ca}^{2+}$ channels open, a microdomain of increased $\left[\mathrm{Ca}^{2+}\right]_{\mathrm{i}}$ builds up around the channel pore, being spatially restricted by $\mathrm{Ca}^{2+}$ buffering molecules and diffusional delays (Neher, 1998; Fig. 2, left top panel). With time, the $\mathrm{Ca}^{2+}$ signal will spread out and reach those vesicles in close proximity to the $\mathrm{Ca}^{2+}$ channels earlier than those vesicles located further away. The higher $\left[\mathrm{Ca}^{2+}\right]_{i}$ at the $\mathrm{Ca}^{2+}$ sensor is, the faster the fusion reaction will be (Schneggenburger and Neher, 2000; Bollmann et al., 2000). Thus, different $\left[\mathrm{Ca}^{2+}\right]_{i}$ at vesicles, due to different distances to activated $\mathrm{Ca}^{2+}$ channels, will induce different release probabilities, which finally will lead to positional heterogeneity.

The second class of hypotheses for mechanisms underlying heterogeneous release probabilities is based on intrinsic heterogeneities (Fig. 2, right panels). Intrinsic heterogeneity includes mechanisms, in which the release probability can differ, irrespective 


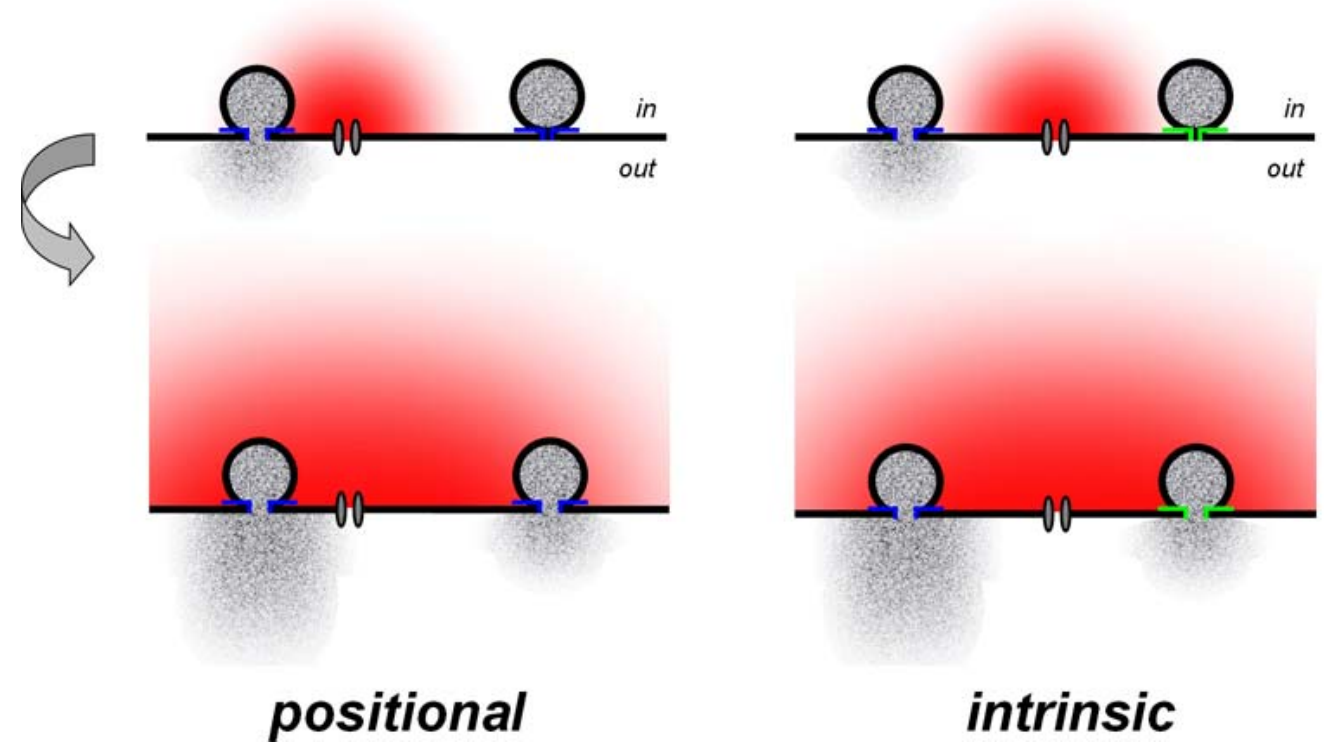

Fig. 2 Positional and intrinsic heterogeneity in release kinetics during presynaptic depolarizations

Cartoon of vesicle fusion triggered by activation of voltage gated $\mathrm{Ca}^{2+}$ channels (vertical rods, $\mathrm{Ca}^{2+}$ microdomain shown in red). In case of positional heterogeneity (left panels), vesicles have the same $\mathrm{Ca}^{2+}$ sensitivity $\left(\mathrm{Ca}^{2+}\right.$ sensors symbolized in blue), being opposite to intrinsic heterogeneity (right panels; $\mathrm{Ca}^{2+}$ sensors with different $\mathrm{Ca}^{2+}$ sensitivities in blue and green). The temporal sequence and concomitant spread out of $\mathrm{Ca}^{2+}$ is indicated (arrow, from top to bottom).

of the intensity of the signal arriving. Such a behavior could have several origins. To become fusion-competent, vesicles need to be docked to the presynaptic membrane, and primed thereafter (for reviews, see Fernández-Chacón and Südhof, 1999; Jahn et al., 2003). Vesicles being primed to different degrees (Jahn et al., 2003) could have different fusion rates (Voets et al., 1999; Xu et al., 1999; Voets, 2000; Sørensen, 2004), which might result either from necessary completion of priming as a prerequisite for fusion, or from an increased energy barrier for fusion from incomplete priming states. Another mechanism to explain intrinsic heterogeneity might be lateral inhibition of vesicle fusion (Triller and Korn, 1985; Stevens and Wang, 1995; Dobrunz et al., 1997; but see also Abanavoli et al., 2002), in the sense that membrane incorporation of fused vesicles leads to cell membrane relaxation, which in turn might increase the energy barrier for subsequent vesicle fusion. Another possibility is that such membrane insertion into the active zone reduces the density of incorporated proteins, thus increasing distances between $\mathrm{Ca}^{2+}$ channels and $\mathrm{Ca}^{2+}$ sensors. But 'intrinsic heterogeneity' might also be explained by differences in the $\mathrm{Ca}^{2+}$ sensitivity of the $\mathrm{Ca}^{2+}$ sensor itself. Probably, different vesicles are equipped with distinct $\mathrm{Ca}^{2+}$ sensors of 
different $\mathrm{Ca}^{2+}$ sensitivities (Sugita et al., 2002). Yet another possibility is that the same $\mathrm{Ca}^{2+}$ sensor can exist in states with different $\mathrm{Ca}^{2+}$ sensitivities.

\section{Aim of this study}

This study aimed at clarifying the mechanism underlying heterogeneous release probabilities, and, in particular, to distinguish between the relevance of positional or intrinsic heterogeneity at the calyx of Held synapse. For this purpose, the major approach used was presynaptic $\mathrm{Ca}^{2+}$ uncaging to elevate $\left[\mathrm{Ca}^{2+}\right]_{\mathrm{i}}$ to a controlled and spatially homogeneous level, combined with simultaneous presynaptic $\left[\mathrm{Ca}^{2+}\right]_{\mathrm{i}}$ measurements and postsynaptic current recordings. Spatially homogeneous elevations in $\left[\mathrm{Ca}^{2+}\right]_{\mathrm{i}}$ by $\mathrm{Ca}^{2+}$ uncaging deliberately avoided $\left[\mathrm{Ca}^{2+}\right]_{\mathrm{i}}$ gradients, which arise during a physiological depolarizing stimulus. Therefore, this approach ruled out influences of inhomogeneous distances between $\mathrm{Ca}^{2+}$ channels and $\mathrm{Ca}^{2+}$ sensors, since $\mathrm{Ca}^{2+}$ channels are not involved in stimulation, here. Thus, if $\mathrm{Ca}^{2+}$ uncaging still leads to heterogeneous release probabilities, and if refilling cannot account for the appearance of a slow release component, the observed biphasic release should not be due positional heterogeneity, but rather have an intrinsic origin.

For $\mathrm{Ca}^{2+}$ uncaging and $\left[\mathrm{Ca}^{2+}\right]_{i}$ measurements, the $\mathrm{Ca}^{2+}$ chelator and the $\mathrm{Ca}^{2+}$ indicator dye were introduced into the presynaptic terminal in whole-cell patch-clamp recordings (Neher and Sakmann, 1976). Simultaneous pre- and postsynaptic voltage-clamp recordings (Forsythe et al., 1995; Borst et al., 1995) allowed for measurement of postsynaptic excitatory currents (EPSCs), which could be elicited either by presynaptic $\mathrm{Ca}^{2+}$ uncaging, or by presynaptic depolarizations. Deconvolution of an EPSC yielded the time course of the presynaptic release rate (Neher and Sakaba, 2001; Sakaba and Neher, 2001a), and therefore this methods allowed for detailed, and quantitative studies of the presynaptic release time course to test for distinct presynaptic release probabilities. 


\section{Methods}

\subsection{Brain slice preparation}

The experimental approach to record from acute brain slices is based on a procedure established by Edwards et al. (1989). 8 to 10 day old Wistar rats were decapitated, and the head was immediately immersed with a carbogen $\left(95 \% \mathrm{O}_{2}, 5 \% \mathrm{CO}_{2}\right)$ saturated preparation solution (see below) of $0^{\circ} \mathrm{C}$. The low temperature and a low $\left[\mathrm{Ca}^{2+}\right]_{\mathrm{i}}(0.1 \mathrm{mM})$ was chosen to down-regulate metabolic processes, and to avoid hypoxic damage of the nerve cells. Having removed the cranial calotte, a transversal cut with the scalpel separated the caudal part of the cerebrum from its rostral part including the optic lobes. Successively severing lateral and ventral nerves from rostral to caudal with forceps detached the caudal part of the brain from the rest of the head. The isolated tissue thus included the caudal cerebrum, the cerebellum, and the brainstem. For smoother cutting of slices, the pia mater and arachnoidea were removed from the ventral surface of the brainstem with forceps without damaging the tissue below.

For an advantageous orientation of the tissue during slicing, the remaining cerebrum was cut at an angle of about $20^{\circ}$ to the midsagittal plane (Fig. 3, thick grey line), and the intersection then was glued (UHU Sekundenkleber, UHU, Germany) onto the Teflon block of a slicing chamber. The chamber was flooded with carbogen saturated preparation solution, and inserted into the slicer (752M Vibroslice, Campden Instruments, Loughborough, UK). On the ventral surface of the brainstem, the brighter area of the trapezoid body served as a landmark for the first transversal cut through the brainstem. The MNTB was included in the next $4-6$ slices of $200 \mu \mathrm{m}$ thickness (Fig. 3, thin grey lines). Each slice was immediately transferred into a carbogen-perfused standard ringer solution (see below) of $37^{\circ} \mathrm{C}$, and incubated there for at least 30 minutes to maximally 5 hours.

For electrophysiological and optical recordings, a slice-chamber with an inner volume of about $0.7 \mathrm{ml}$ was custom-build, and the slice was fixed there with a harp, made of stretched nylon strings glued onto a $\Pi$ shaped platinum wire. The slice was continuously perfused with a flow rate of about $1-1.5 \mathrm{ml} / \mathrm{min}$, and the extracellular ringer solution (see below) could be changed according to the experimental design. A complete exchange of the extracellular solution was accomplished within one to two minutes. 


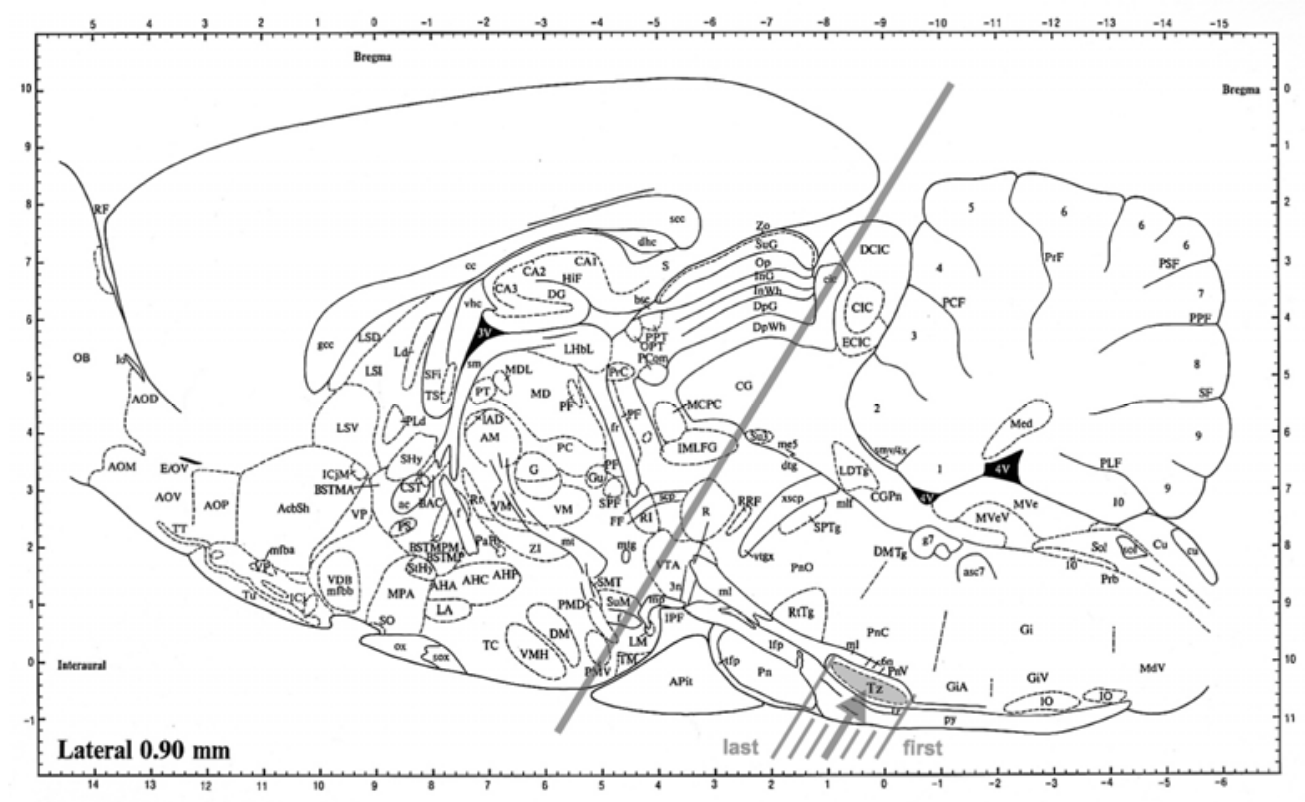

Fig. 3 MNTB slice preparation in the rat brainstem

Lateral view of the adult rat brain (rostral to caudal from left to right), at $0.90 \mathrm{~mm}$ from ipsilateral (taken from Paxinos and Watson, 1986). Positions for transverse sections are indicated: first, a scalpel cut dissects the brainstem from the cerebrum (thick grey line), and then slices are obtained with vibratom cuts (parallel thin grey lines) in the MNTB region ("Tz", marked with arrow).

\section{Solutions}

Extracellular solutions were bubbled with carbogen $\left(95 \% \mathrm{O}_{2}, 5 \% \mathrm{CO}_{2}\right)$ adjusting $\mathrm{pH}$ to 7.4 , and osmolarity was set to about 300 mOsm. Since bicarbonate also buffers free $\mathrm{Ca}^{2+}$ (apparent dissociation constant $\mathrm{K}_{\mathrm{D} \text {, app }} \approx 160 \mathrm{mM}$; Martell and Smith, 1974), the concentration of free $\mathrm{Ca}^{2+}$ was a lower than the nominal concentration (about $1.6 \mathrm{mM}$, Schneggenburger et al., 1993; Marciani et al., 1982).

For brain preparation, a cooled standard ringer solution with low $\left[\mathrm{Ca}^{2+}\right]_{\mathrm{i}}(0.1 \mathrm{mM})$ was used (Table 2-1). Before preparation of the slices, this solution was put into a freezer until a few ice crystals appeared. The low temperature and low $\left[\mathrm{Ca}^{2+}\right]_{i}$ helped to minimize cellular activity and to reduce damage of the neuronal tissue. For incubation of the slices before and during experiments, a standard ringer solution (Table 2-1) was used. The solution was heated to $37^{\circ} \mathrm{C}$ for incubation, and experiments were performed at room temperature $\left(21^{\circ} \mathrm{C}-\right.$ $26^{\circ} \mathrm{C}$ ). 


\begin{tabular}{|c|c|c|}
\hline \multirow[b]{3}{*}{ substance } & \multicolumn{2}{|c|}{ extracellular solutions } \\
\hline & \multirow[t]{2}{*}{ standard ringer } & \multirow[t]{2}{*}{ preparation ringer } \\
\hline & & \\
\hline $\mathrm{NaCl}$ & 125 & 125 \\
\hline $\mathrm{NaHCO}_{3}$ & 25 & 25 \\
\hline $\mathrm{NaH}_{2} \mathrm{PO}_{4}$ & 1.25 & 1.25 \\
\hline $\mathrm{KCl}$ & 2.5 & 2.5 \\
\hline glucose & 25 & 25 \\
\hline myoinositol & 3 & 3 \\
\hline Na-pyruvate & 2 & 2 \\
\hline ascorbic acid & 0.4 & 0.4 \\
\hline $\mathrm{CaCl}_{2}$ & 2 & 0.1 \\
\hline $\mathrm{MgCl}_{2}$ & 1 & 3 \\
\hline $\mathrm{pH}$ (bubbled with carbogen) & \multicolumn{2}{|c|}{7.4} \\
\hline osmolarity (mOsm) & \multicolumn{2}{|c|}{300} \\
\hline
\end{tabular}

Table 2-1 Extracellular solutions (concentrations in $\mathrm{mM}$ )

During experiments, the extracellular solution also contained $10 \mathrm{mM}$ TEA-Cl and $1 \mu \mathrm{M}$ TTX (Alamone labs, Jerusalem, Israel) to block $\mathrm{Na}^{+}$and $\mathrm{K}^{+}$channels, $50 \mu \mathrm{M}$ D-AP5 (D-(-)-2amino-5-phosphonopentanoic acid; Tocris, Bristol, UK) to block NMDA type glutamate receptors, $100 \mu \mathrm{M} \mathrm{CTZ} \mathrm{(cyclothiazide;} \mathrm{Tocris)} \mathrm{to} \mathrm{minimize} \mathrm{postsynaptic} \mathrm{AMPA} \mathrm{receptor}$ desensitization (Trussel et al., 1993; Otis et al., 1996), and, if not stated differently, $2 \mathrm{mM} \gamma$ DGG (Tocris) to competitively block postsynaptic AMPA type glutamate receptors (Watkins and Evans 1981; Jones et al., 1984), and therefore to prevent AMPA-receptor saturation (Neher and Sakaba, 2001; Sun and Wu, 2001).

$\mathrm{NaCl}, \mathrm{NaHCO}_{3}, \mathrm{NaH}_{2} \mathrm{PO}_{4}, \mathrm{KCL}, \mathrm{CaCl}_{2}$, and $\mathrm{MgCl}_{2}$ were purchased from Merck (Darmstadt, Germany), and $\mathrm{D}(+)$-glucose, myoinositol, Na-pyruvate, and L-ascorbic acid from Sigma (Steinheim, Germany). 


\subsection{Whole-cell patch-clamp recordings}

For electrophysiological recordings, an EPC-9/2 patch-clamp amplifier (C-board version, HEKA) was used. Electrical currents were sampled on a Macintosh Quadra 950 computer (256 MB RAM) using the software Pulse (V8.50 with lock-in extension, HEKA) running on a Mac OS D2-9.1 operating system. For further analysis, the data was transferred to a Dell Optiplex GX260 PC (2.4 GHz, 512 MB RAM; Dell), and loaded into the analysis software Igor Pro (Wavemetrics Inc., Lake Oswego OR, USA).

The two headstages containing the preamplifiers were mounted on piezoelectric micromanipulators (Piezosystems Jena, Jena, Germany), and the coupled pipette holders were airtight connected to air pressure sensors, and a mouthpiece for oral pressure adjustment. $\mathrm{Ag} / \mathrm{AgCl}$ electrodes connected the preamplifiers with the intracellular solution in the patch pipette, and via a bath electrode with the extracellular solution.

Patch-pipettes were pulled from borosilicate capillaries $(2 \mathrm{~mm}$ diameter, $0.3 \mathrm{~mm}$ wall thickness) with a vertical two-step pipette puller (HEKA Electronik, Lambrecht/Pfalz, Germany). The heat-polished pipette tips had a diameter of about $1 \mu \mathrm{m}$, giving a pipette resistance of $4.0-5.5 \mathrm{M} \Omega$, and $2.5-3.5 \mathrm{M} \Omega$ for pre- and postsynaptic recordings, respectively. To minimize the pipette capacitance, and to slow down capacitive transients for easier software capacitance compensation, the outer pipette wall was covered with an isolating synthetic resin (Sylgard Silicon RTV, Sinus Electronics $\mathrm{GmbH}$, Untereisesheim, Germany; Edwards et al., 1989).

Calyces of Held were identified under infrared illumination, and also fluorescence images of $\mathrm{Ca}^{2+}$ indicator dye filled presynaptic terminals confirmed correct identifications. For paired pre- and postsynaptic recordings, pipettes were filled with the according intracellular solutions (see below), and since high concentrations of the $\mathrm{Ca}^{2+}$ chelator DM-nitrophen (DMN; chapter 2.3) make sealing difficult, presynaptic pipettes were dipped into a DMN-free intracellular solution, and then filled from the back with the DMN-containing solution. Slight pressure was applied to the pipette when approaching the cell, being smaller for presynaptic recordings, in order to prevent the dipping solution without DMN to be blown out too quickly. Releasing the pressure, or for the presynapse an abrupt change to slightly negative values, led to tight and stable seals reaching a resistance of several $G \Omega$. Having obtained a $G \Omega$ seal, short suction pulses ruptured an opening into the cell membrane underneath the tip of the patch pipette. The achieved access resistance was about two times larger than the mere pipette resistance (Marty and Neher, 1995), being $8-25 \mathrm{M} \Omega$ and $3-9 \mathrm{M} \Omega$ for pre- and postsynaptic recordings, respectively. Diffusion-driven exchange of soluble components led 
to a replacement of the cytoplasm by the pipette solution (Pusch and Neher, 1988). In this way, fluorescent dyes, and photolabile Calcium chelators could be introduced into the cell.

During presynaptic sealing, the extracellular fluid was changed from standard to the wanted toxic composition, and after attaining paired whole-cell configurations, stimulation protocols were started with a delay of at least 3 minutes. This allowed intra- and extracellular toxins fully to take effect, and all components of the artificial intracellular solutions to properly washin.

For cellular recovery from activity, stimuli where separated by at least one minute for short depolarizations up to $16 \mathrm{~ms}$, at least 1.5 minutes for depolarizations up to $50 \mathrm{~ms}$, and at least 2 minutes for all other stimuli including $\mathrm{Ca}^{2+}$ uncaging.

Recordings were taken only as long as the leak current was smaller than $100 \mathrm{pA}$ for presynaptic, or $500 \mathrm{pA}$ for postsynaptic recordings, typically ranging between $50 \mathrm{pA}$ and 80 $\mathrm{pA}$, and between $100 \mathrm{pA}$ and $300 \mathrm{pA}$, respectively. Also, rundown of cellular activity was always checked by comparison of peak EPSC amplitudes and presynaptic pool size estimates from deconvolved postsynaptic traces (chapter 2.4.1). As soon as the amount of vesicles released decreased compared to stimuli of similar intensity, data was discarded.

To compensate for charging transients of the pipette wall, and of the cell membrane, both were corrected for by using the internal, software controlled compensation circuits of the EPC-9/2 amplifier. In the cell-attached configuration, the pipette capacitance and series resistance could nicely be equilibrated, and in the whole-cell mode the cell membrane capacitance and membrane resistance were compensated, too. To correct for membrane voltage errors due to high access resistances to the cell, the automatic EPC-9/2 $R_{\mathrm{s}^{-}}$ compensation has been used (time constant of $10 \mu \mathrm{s}$ ), and was set to $50 \%$ for presynaptic, and to $50 \%$ - $90 \%$ for postsynaptic recordings. The recorded traces were corrected for this off-line. Also, charging transients, arising when the membrane potential was changed, were estimated using a standard $\mathrm{P} / 4$ protocol for each stimulus applied, and presynaptic passive components were subtracted off-line.

Under whole-cell voltage-clamp conditions, membrane currents were sampled at $50 \mathrm{kHz}$, the holding potential was $-80 \mathrm{mV}$ presynaptically, and $-70 \mathrm{mV}$ postsynaptically. For presynaptic sine+DC membrane capacitance measurements, a $2 \mathrm{kHz}, 70 \mathrm{mV}$ peak-to-peak sine wave was applied in the amplifier's lock-in mode (Wölfel et al. 2003). 
Depolarizing presynaptic stimuli always clamped the presynaptic voltage to $0 \mathrm{mV}$ for efficient $\mathrm{Ca}^{2+}$ entry into the cell through voltage-gated $\mathrm{Ca}^{2+}$ channels. In many cases, steps to $0 \mathrm{mV}$ were preceded by a $4 \mathrm{~ms}$ jump to $+80 \mathrm{mV}$ to open the $\mathrm{Ca}^{2+}$ channels at a voltage, where the driving force for $\mathrm{Ca}^{2+}$ to enter the cell is low. Thus, without triggering vesicle release beforehand, $\mathrm{Ca}^{2+}$ entry became more efficient for a subsequent step to $0 \mathrm{mV}$.

\section{Intracellular solutions}

The production of the intracellular solutions was partitioned into two steps. First, a two times concentrated stock solution without $\mathrm{CaCl}_{2}, \mathrm{MgCl}_{2}$, Fura-2FF or DMN was made for storage up to three month at $-20^{\circ} \mathrm{C}$. Using this stock solution, the final intracellular solution was mixed every one to two weeks, adding the other components remaining.

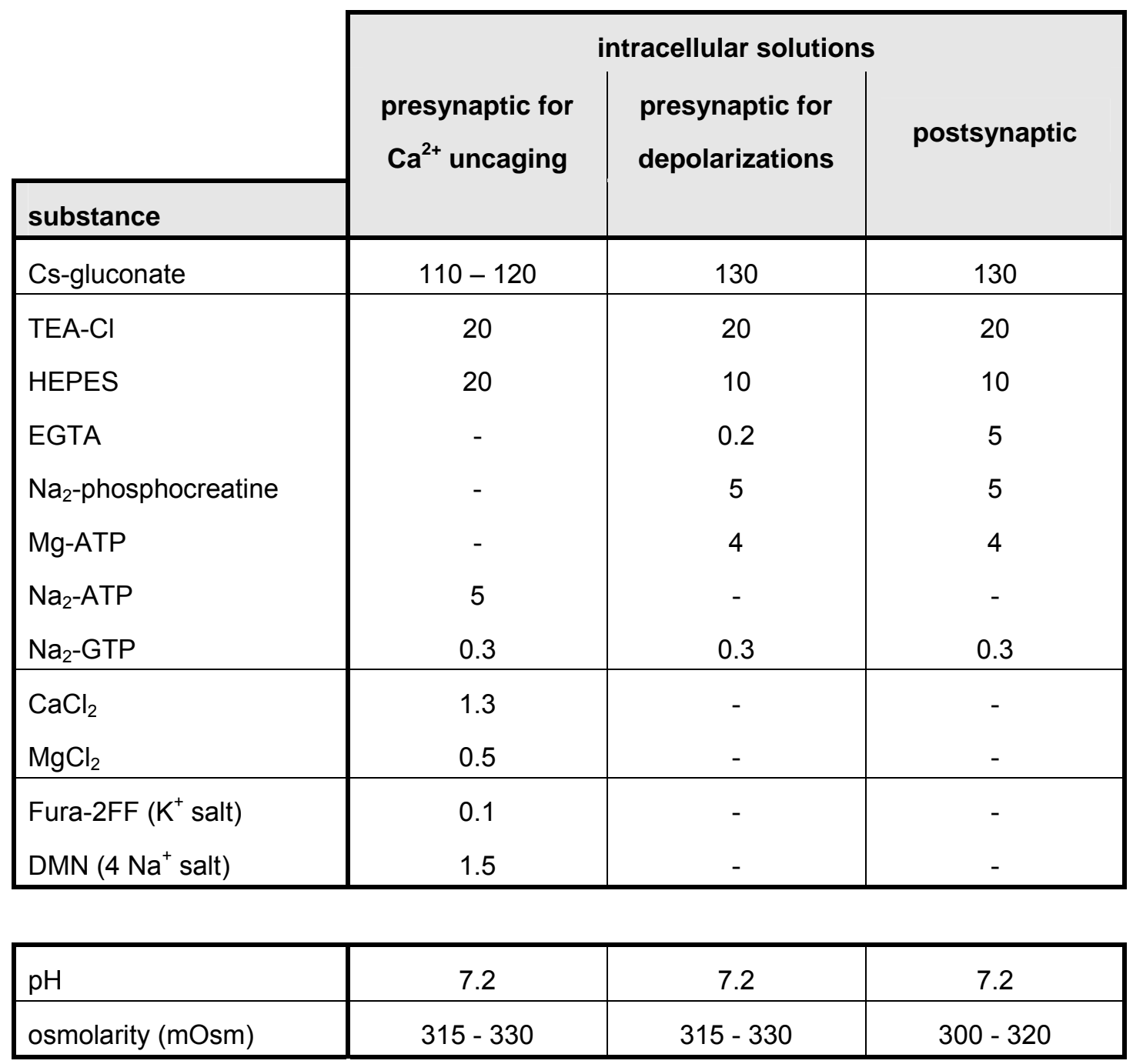

Table 2-2 Intracellular solutions (concentrations in $\mathbf{m M}$ ) 
Cesium-gluconate was not commercially available, and therefore self-made according to Meyer (1999). $\mathrm{CaCl}_{2}$ and $\mathrm{MgCl}_{2}$ were purchased from Merck (Darmstadt, Germany), Fura2FF ( $\mathrm{K}^{+}$salt) from Tef-Labs (Austin, UK), and DMN (4 Na+ salt) from Calbiochem (Darmstadt, Germany). All other chemicals were bought from Sigma (Steinheim, Germany).

\section{$2.3 \mathrm{Ca}^{2+}$ uncaging and $\mathrm{Ca}^{2+}$ imaging}

Visualization of the cells was achieved with an upright microscope (Axioskop FS, Zeiss, Germany), equipped with a Dodt contrast-gradient infrared illumination (Luigs und Neumann, Ratingen, Germany), a 60x water-immersion objective (numerical aperture 0.9, Olympus, Germany), and a TILL Imago CCD camera with associated visualization software (TILLvisION, T.I.L.L. photonics $\mathrm{GmbH}$, Gräfelfing, Germany).

For UV photolysis of the $\mathrm{Ca}^{2+}$ chelator DM-nitrophen (Calbiochem, La Jolla CA, USA), a SP20 flash lamp (Dr. Gert Rapp Optoelektronik, Wedel, Germany) was used. With a capacitance of $740 \mu \mathrm{F}$, and a charging potential of $750 \mathrm{~V}$, the flash pulse energy was set to maximal, and the evoked flashlight was filtered with a UV-filter (low pass below $390 \mathrm{~nm}$ ). Using neutral density filters (Linos AG, Göttingen, Germany) of different optical densities $(50 \%, 32 \%, 20 \%, 10 \%)$ the flash intensity could be diminished, thus raising $\left[\mathrm{Ca}^{2+}\right]_{i}$ to controlled levels. A quartz light-guide connected the flash lamp with the epi-fluorescence entry of the microscope, and the illumination window was set to $30 \mu \mathrm{m} * 30 \mu \mathrm{m}$, large enough to expose a whole presynaptic terminal to the flash light.

The visualization system by T.I.L.L. photonics included a slow-scan CCD camera with an interline-transfer chip (Sony, Japan) and a frame-grabber card, and the imaging software TILLvisION 4.0 installed on a Dell Precision PC (2.8 GHz, 512 MB RAM; Dell, Germany), running on a Microsoft Windows 2000 operating system. The image from the specimen could optionally be projected through the microscope's ocular, or to the camera.

The excitation light for the $\mathrm{Ca}^{2+}$ indicator dye was generated by a Polychrome II monochromator (T.I.L.L. photonics) with a rapid wavelength selection implementation: part of the spectrum of a xenon short arc lamp (Ushio Inc., Japan) was transmitted via a diffractional grating within the monochromator into a quartz light-guide. This light guide was connected to the epi-fluorescence entry of the microscope. For simultaneous $\mathrm{UV} \mathrm{Ca}^{2+}$ uncaging, and $\left[\mathrm{Ca}^{2+}\right]_{i}$ measurements, a dichroic mirror passed both excitation lights to the tissue. The UVflashlight to photolyse the $\mathrm{Ca}^{2+}$ chelator passed the dichroic mirror into the epi-fluorescence entry by $85 \%$ (40\%), while the light from the monochromator to excite the $\mathrm{Ca}^{2+}$ indicator dye 
coming from the side was reflected by the dichroic mirror into the epi-fluorescence entry by $15 \%(60 \%)$.

Under epi-fluorescence conditions, excitation and emission light of the $\mathrm{Ca}^{2+}$ indicator dye passes the objective. Thus, the light had to be split into two spectral bands by another dichroic mirror, to separate excitation before, and emission light after passing through the objective. For measurements with Fura-2FF, the dichroic mirror used reflected excitation light below $420 \mathrm{~nm}$ wavelength into the objective to the tissue, while emission light above $420 \mathrm{~nm}$ could pass the mirror towards the detection camera.

Control and synchronization of flash lamp, monochromator, camera, and frame grabber, and synchronized triggering of electrophysiological equipment was achieved with the visualization software.

\section{Imaging protocol}

For $\mathrm{Ca}^{2+}$ uncaging experiments, a UV flash-photolysis uncaging stimulus was given $100 \mathrm{~ms}$ after onset of the electrophysiological recordings (Fig. 4, at $\mathrm{t}=0$ ). The fluorescent images needed for an estimate of the presynaptic $\left[\mathrm{Ca}^{2+}\right]_{\mathrm{i}}$ (see below) were taken with an $8 * 15$ binning of the 640 * 480 pixel area (width * height), resulting in a image size of 80 * 32 pixels. Together with an illumination time of $5 \mathrm{~ms}$ for each frame taken, this resulted in an acceptable signal-to-noise ratio. A maximal signal was gained at basal $\left[\mathrm{Ca}^{2+}\right]_{\mathrm{i}}$ (about $100 \mathrm{nM}$ ) and $5 \mathrm{~ms}$ exposure of $380 \mathrm{~nm}$ excitation light. With a bit depth of 4096 shades of grey, here the brightest pixels had a relative intensity of $1000-2500$.

Before a $\mathrm{Ca}^{2+}$ uncaging stimulus was given, a single image ratio for an estimate of basal $\left[\mathrm{Ca}^{2+}\right]_{\mathrm{i}}$ has been taken (Fig. 4, $\mathrm{t}<0$ ), and after the UV flash, a series of images was recorded in a fast sequence (Fig. $4, \mathrm{t}>0$ ). To improve temporal resolution of $\left[\mathrm{Ca}^{2+}\right]_{i}$ measurements immediately after a $\mathrm{Ca}^{2+}$ uncaging stimulus, four images only at $380 \mathrm{~nm}$ excitation wavelength were taken (Fig. 4, black traces). This was also possible, because the fluorescence intensity at $350 \mathrm{~nm}$ did not change significantly directly after the uncaging stimulus, so that all four frames at $380 \mathrm{~nm}$ could be correlated to the subsequent one at 350 $\mathrm{nm}$. When switching between two wavelengths, an intermission of $2 \mathrm{~ms}$ was required by the system, so that the effective frame rate summed up to totally $1 / 7 \mathrm{kHz}$. When staying at the same wavelength an inter-frame interval of $1 \mathrm{~ms}$ was needed, giving a frame rate of $1 / 6 \mathrm{kHz}$. Thus, during fast $\left[\mathrm{Ca}^{2+}\right]_{\mathrm{i}}$ measurements, the four initial images taken at $380 \mathrm{~nm}$ wavelength yielded a $\left[\mathrm{Ca}^{2+}\right]_{i}$ measurement rate of $1 / 6 \mathrm{kHz}$. At all other times, pairs of $380 \mathrm{~nm}$ and $350 \mathrm{~nm}$ images were taken, so that the inter-frame interval of $2 \mathrm{~ms}$ plus the exposure time of $5 \mathrm{~ms}$ for each of the two images taken summed to a final rate of $1 / 14 \mathrm{kHz}$. 


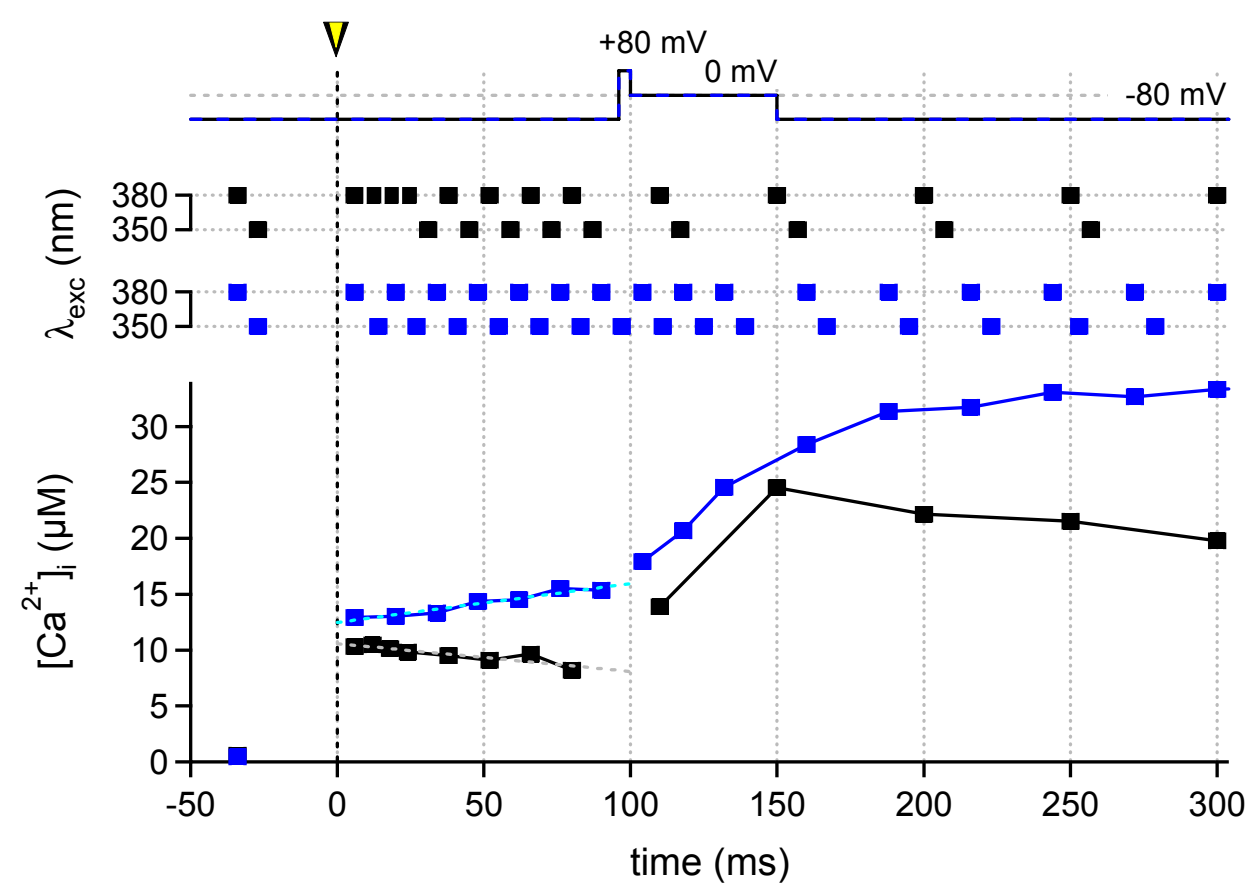

Fig. 4 Imaging protocol for $\mathrm{Ca}^{2+}$ uncaging experiments

Two $\mathrm{Ca}^{2+}$ uncaging stimuli (at $\mathrm{t}=0$; dashed black line at yellow arrow) in two different cells (black and blue traces, respectively) increased $\left[\mathrm{Ca}^{2+}\right]_{i}$ to $10-15 \mu \mathrm{M}$. Fluorescent images $(\lambda=$ $350 \mathrm{~nm}$ and $380 \mathrm{~nm}$ ) were taken before, and after stimulation (stimulus at $t=0$, yellow arrowhead). $100 \mathrm{~ms}$ after $\mathrm{Ca}^{2+}$ uncaging, a $50 \mathrm{~ms}$ depolarization (uppermost traces) further increased $\left[\mathrm{Ca}^{2+}\right]_{\text {i. }}$ Changes in $\left[\mathrm{Ca}^{2+}\right]_{i}$ after $\mathrm{Ca}^{2+}$ uncaging were analyzed by linear regressions (dashed grey and blue lines on $\left[\mathrm{Ca}^{2+}\right]_{\mathrm{i}}$ traces).

Due to internal properties of the imaging system, the high intensity flashlight corrupted all data sampled between $16 \mathrm{~ms}$ before and $4 \mathrm{~ms}$ after the flash. Thus, the earliest midpoint of the first $380 \mathrm{~nm}$ frame was located $6.5 \mathrm{~ms}$ after the flash.

To extract the global presynaptic $\left[\mathrm{Ca}^{2+}\right]_{i}$ from the time series of images recorded, the fluorescence intensities of six bright pixels in the area of the presynaptic terminal were corrected for background fluorescence, which in turn was collected outside the presynaptic terminal, but inside the flash window. The fluorescence intensities were then processed as described below.

For $\left[\mathrm{Ca}^{2+}\right]_{i}$ dose-response curves, $\left[\mathrm{Ca}^{2+}\right]_{i}$ at the $\mathrm{Ca}^{2+}$ uncaging stimulus was read out. This was done by fitting a linear regression to the first four $\left[\mathrm{Ca}^{2+}\right]_{i}$ estimates after the stimulus, and then extrapolating this regression back to onset of stimulation. The back-extrapolated $\left[\mathrm{Ca}^{2+}\right]_{\mathrm{i}}$ at this point of time was defined as the stimulus intensity. 


\section{Controlled liberation of $\mathrm{Ca}^{2+}$ by $\mathrm{Ca}^{2+}$ uncaging}

To raise $\left[\mathrm{Ca}^{2+}\right]_{\mathrm{i}}$ in a controlled manner, the photolabile $\mathrm{Ca}^{2+}$ chelator DMN (Kaplan and EllisDavies, 1988; Ellis-Davies et al., 1996) was introduced into the presynaptic terminal via the patch pipette. Excitation with light of about $350 \mathrm{~nm}$ wavelength splits the chelator molecule with a half-time of about $10-20 \mu$ s (Ellis-Davies et al., 1996), so that the high apparent dissociation constant decreases markedly ( $\mathrm{K}_{\mathrm{D}}$ from ca. $5 \mathrm{nM}$ to ca. $3 \mathrm{mM}$; Kaplan and EllisDavies, 1988; McCray et al., 1992), and the concentration of free $\mathrm{Ca}^{2+}$ increases, correspondingly. Thus by variation of UV light intensity, $\left[\mathrm{Ca}^{2+}\right]_{i}$ could be elevated to a controlled degree. To keep the $\mathrm{Ca}^{2+}$ buffering capacities at rest close to physiological conditions, the $\mathrm{Ca}^{2+}$ chelator molecules were loaded with $\mathrm{Ca}^{2+}$ to a high extend. Because DMN shows a to $\mathrm{Ca}^{2+}$ competitive affinity to Magnesium ions $\left(\mathrm{Mg}^{2+}\right.$; Hyrc et al., 2000), the $\mathrm{Mg}^{2+}$ concentration was kept low, not to reduce the efficiency of $\mathrm{Ca}^{2+}$ liberation from DMN. This technique has already been applied to nerve terminals of the CNS (Heidelberger et al., 1994; Schneggenburger and Neher, 2000; Bollmann et al., 2000).

\section{Measurement of the intracellular Calcium concentration}

To estimate $\left[\mathrm{Ca}^{2+}\right]_{\mathrm{i}}$ in the presynaptic terminal, the ratiometric $\mathrm{Ca}^{2+}$ indicator dye Fura-2FF $\left(\mathrm{K}^{+}\right.$salt; Tef-Labs, Austin, UK) was used. The excitation spectrum of this fluorescent dye shifts in dependence on $\left[\mathrm{Ca}^{2+}\right]_{\mathrm{i}}$, and this shift can be monitored by ratiometric measurements of fluorescence intensity at different wavelengths. The comparison of fluorescence, measured at different wavelength of excitation light, makes the estimate of $\left[\mathrm{Ca}^{2+}\right]_{\mathrm{i}}$ independent of unknown parameters, such as the exact dye concentration, or the layer thickness of the illuminated specimen producing filter effects. Therefore, a quantitative estimate of $\left[\mathrm{Ca}^{2+}\right]_{i}$ can be made (Grynkiewiecz et al., 1985):

$$
\left[\mathrm{Ca}^{2+}\right]_{i}=K_{\text {eff }} \cdot \frac{\left(R-R_{\min }\right)}{\left(R_{\max }-R\right)}
$$

Here, $K_{\text {eff }}$ is the effective dissociation constant, and $R$ is the ratio of fluorescence intensities at two different excitation wavelengths. In this study, for Fura-2FF $\left(K_{D} \approx 6.7 \mu M\right.$; see also chapter 2.3.1) excitation wavelengths of $350 \mathrm{~nm}$ and $380 \mathrm{~nm}$ were used:

$$
R=\frac{F_{350}}{F_{380}}
$$


To calibrate the system (chapter 2.3.1), the lower boundary of this ratio $R_{\min }$ was determined at total absence of $\mathrm{Ca}^{2+}$, and the upper limit $R_{\max }$ was extracted by completely saturating the indicator dye with exceedingly high concentrations of $\mathrm{Ca}^{2+}$.

\subsubsection{Calibration of the Calcium concentration measurements}

To estimate the absolute $\left[\mathrm{Ca}^{2+}\right]_{\mathrm{i}}$ in a cell, three calibration constants have to be known: $R_{\text {min }}$, $R_{\max }$, and $K_{\text {eff }}$ (see above). These are apparatus constants, depending on properties of the monochromator light, components in the optic pathways, the camera, filter effects of the extracellular solution, and the composition of the intracellular solution including the fluorescent indicator dye. Thus, the conditions for the calibration have to be the same as in the corresponding experiment, and changes in the experimental conditions necessitate a new calibration. The only difference in the calibration solution to those in the experiments was the addition of $\mathrm{Ca}^{2+}$ buffers (EGTA with $\mathrm{K}_{\mathrm{D}} \approx 180 \mathrm{nM}$, and DPTA with $\mathrm{K}_{\mathrm{D}} \approx 80 \mu \mathrm{M}$; Naraghi, 1997) to clamp $\left[\mathrm{Ca}^{2+}\right]_{i}$ to the desired level.

A difference between in vivo and in vitro calibrations only showed up in the estimate of $R_{\min }$, but the effects of the in vitro estimate of this parameter on the finally calculated $\left[\mathrm{Ca}^{2+}\right]_{i}$ using Fura-2FF as indicator was negligible for the purposes of this study. Therefore, here the less difficult and time-consuming in vitro calibrations were chosen.

For each experimental setting, a five-point calibration has been made, including one solution with no $\mathrm{Ca}^{2+}$ to calculate $R_{\min }$, one solution with an excessive $\left[\mathrm{Ca}^{2+}\right]_{\mathrm{i}}$ saturating all $\mathrm{Ca}^{2+}$ buffers to calculate $R_{\max }$, one solution with a free $\left[\mathrm{Ca}^{2+}\right]_{\mathrm{i}}$ of about $11 \mu \mathrm{M}$ close to the $\mathrm{K}_{\mathrm{D}}$ of Fura-2FF, and two other solutions with free $\left[\mathrm{Ca}^{2+}\right]_{i}$ close to the intermediate $\left[\mathrm{Ca}^{2+}\right]_{i}$ to check calibration quality. Principally, only a three-point calibration would be necessary, but with five-point calibrations, as made here, erroneous mixing of the calibration solutions becomes more apparent. The detailed compositions of the three solutions mentioned first $\left(R_{\min }, R_{\max }\right.$, and the intermediate $R$ ) are listed in Table 2-1. The free $\left[\mathrm{Ca}^{2+}\right]_{\mathrm{i}}$ has been calculated with a self-written macro (FreCon V3.1beta, written by Tao Xu and Jacob Sørensen) for Igor Pro software (V4.01; Wavemetrics Inc., Lake Oswego OR, USA). This macro took absolute concentrations of all $\mathrm{Ca}^{2+}$ buffers present in the presynaptic pipette solution into account, and moreover, calculated free $\mathrm{Ca}^{2+}$ concentrations in dependence of $\mathrm{Ca}^{2+}$ uncaging efficiency. 


\begin{tabular}{|c|c|c|c|}
\hline \multirow[b]{3}{*}{ substance } & \multicolumn{3}{|c|}{ calibration solutions for } \\
\hline & \multirow[t]{2}{*}{$\mathbf{R}_{\min }$} & \multirow[t]{2}{*}{$\mathbf{R}$} & \multirow[t]{2}{*}{$\mathbf{R}_{\max }$} \\
\hline & & & \\
\hline Cs-gluconate & $125-130$ & $125-130$ & $125-130$ \\
\hline TEA-Cl & 20 & 20 & 20 \\
\hline HEPES & 20 & 20 & 20 \\
\hline $\mathrm{Na}_{2}-\mathrm{ATP}$ & 5 & 5 & 5 \\
\hline $\mathrm{Na}_{2}$-GTP & 0.3 & 0.3 & 0.3 \\
\hline $\mathrm{CaCl}_{2}$ & - & 1.5 & 10 \\
\hline $\mathrm{MgCl}_{2}$ & 0.5 & 0.5 & 0.5 \\
\hline EGTA & 10 & 10 & - \\
\hline DPTA & 30 & 30 & - \\
\hline
\end{tabular}

\begin{tabular}{|l|l|l|l|}
\hline Fura-2FF & 0.1 & 0.1 & 0.1 \\
\hline DMN & 1.5 & 1.5 & 1.5 \\
\hline
\end{tabular}

\begin{tabular}{|l|c|c|c|}
\hline $\mathrm{pH}$ & 7.2 & 7.2 & 7.2 \\
\hline osmolarity (mOsm) & $320-330$ & $320-330$ & $320-330$ \\
\hline
\end{tabular}

\begin{tabular}{|l|c|c|c|}
\hline free $\mathrm{Ca}^{2+}$-concentration & 0 & 0.010 & 5.050 \\
\hline
\end{tabular}
\begin{tabular}{|l|c|c|c|}
\hline \multicolumn{3}{|l|}{} \\
$\mathrm{F}_{350} / \mathrm{F}_{380}$ & 0.46 & 1.26 & 7.70 \\
\hline
\end{tabular}

Table 2-3 Calibration solutions for $\left[\mathrm{Ca}^{2+}\right]_{\mathrm{i}}$ measurements (concentrations in $\mathrm{mM}$ )

While the standard calibration solutions were based on the compositions of the intracellular solutions, for validation of the estimate of $K_{\text {eff }}$ an additional solution of completely different composition, but the same free $\left[\mathrm{Ca}^{2+}\right]_{\mathrm{i}}$ as the intermediate at $10 \mu \mathrm{M}$, was made $(80 \mathrm{mM} \mathrm{Cs}-$ DPTA, $10.3 \mathrm{mM} \mathrm{CaCl}_{2}, 20 \mathrm{mM}$ HEPES, 0.1 mM Fura-2FF, $1.5 \mathrm{mM}$ DMN; pH 7.2 and 320330 mOsm; DPTA: 1,3-Diamino-2-propanol-N,N,N',N'-tetraacetic acid; EGTA: Ethylene glycol-bis(2-aminoethylether)-N,N,N',N'-tetraacetic acid; HEPES: N-(2-Hydroxyethyl) piperazine-N'-(2-ethanesulfonic acid)). 
For the in vitro measurements, glass capillaries with a diameter of $50 \mu \mathrm{m}$ (Vitro-Com, New Jersey NJ, USA) were filled with the calibration solutions, and the objective of the microscope was immersed into the respective extracellular solution (chapter 2.1).

As described above, the fluorescence ratios $R$ can directly be calculated from the fluorescence intensities at the given wavelengths (350 and $380 \mathrm{~nm}$ ):

$$
R=\frac{F_{350}}{F_{380}}
$$

With the estimates for $R_{\min }, R_{\max }$ and an intermediate $R$ at a defined free $\left[\mathrm{Ca}^{2+}\right]_{\mathrm{i}}$, the effective dissociation constant $K_{\text {eff }}$ can be calculated as follows:

$$
K_{\text {eff }}=\left[C a^{2+}\right]_{\text {free }} \cdot \frac{\left(R_{\max }-R\right)}{\left(R-R_{\min }\right)}
$$

DMN has effects on the fluorescent properties of the indicator dye (Zucker, 1992), which had to be taken into account when calibrating. These effects arise from differential absorbance of the excitation light by, and additional fluorescence coming from the $\mathrm{Ca}^{2+}$ chelator. Furthermore, these properties differ for the $\mathrm{Ca}^{2+}$ bound chelator, as compared to its photoproducts after photolysis. Thus, the calibration constants were corrected for as described by Heinemann et al. (1994). Calibration solutions always contained the same DMN and Fura-2FF concentrations as during experiments (example in Table 2-3 only for $1.5 \mathrm{mM}$ DMN, others not shown), and the uncaging stimulus in combination with the corresponding imaging protocol for $\left[\mathrm{Ca}^{2+}\right]_{i}$ measurements was applied during calibrations, as well. By this means, different sets of calibration constants were obtained before, and after flash-photolysis.

\subsubsection{Clamping $\left[\mathrm{Ca}^{2+}\right]_{i}$ after $\mathrm{Ca}^{2+}$ uncaging}

The ideal $\mathrm{Ca}^{2+}$ uncaging stimulus is a temporally and spatially homogeneous rise in the presynaptic $\left[\mathrm{Ca}^{2+}\right]_{\mathrm{i}}$. A temporally constant release probability is needed for the whole range of analysis when fitting regressions to the calculated presynaptic cumulative release (chapters 2.4.1 and 2.4.2; several tens of milliseconds). Spatially uniform stimulation intensities are needed to prevent different components of release artificially showing up due to different release probabilities at different locations in the presynaptic terminal. Thus, temporal and spatial inhomogeneities in the $\mathrm{Ca}^{2+}$ uncaging stimulus strength can lead to inhomogeneous 
release rates, which then might be misinterpreted as different release components with intrinsically distinct release probabilities. Therefore, temporal and spatial uniformity of $\left[\mathrm{Ca}^{2+}\right]_{\mathrm{i}}$ after elevation by $\mathrm{Ca}^{2+}$ uncaging was examined.

A decay in $\left[\mathrm{Ca}^{2+}\right]_{i}$ might be caused by $\mathrm{Ca}^{2+}$ exchangers and transporters (for reviews, see Guerini, 1998; Philipson and Nicoll, 2000). To counterbalance such a decay in $\left[\mathrm{Ca}^{2+}\right]_{i}$ for the time of several tens of milliseconds, a few modifications of the experimental design were made. First, the monochromator light has been used to further photolyse $\mathrm{DMN}-\mathrm{Ca}^{2+}$ complexes after the strong UV-flash stimulus. Second, the efficiency of this ongoing $\mathrm{Ca}^{2+}$ uncaging has been improved by increasing the monochromator light intensity at the expense of UV-flashlight intensity, and by increasing the concentration of the $\mathrm{Ca}^{2+}$ chelator. Third, the driving force for $\mathrm{Ca}^{2+}$ extrusion has been decreased by increasing the extracellular $\mathrm{Ca}^{2+}$ concentration $\left(\left[\mathrm{Ca}^{2+}\right]_{0}, 2 \mathrm{mM}\right.$ to $\left.6 \mathrm{mM}\right)$. This should also have increased the basal, intracellular $\mathrm{Ca}^{2+}$ concentration $\left(\left[\mathrm{Ca}^{2+}\right]_{i}\right)$ at rest slightly, concomitantly increasing the $\mathrm{Ca}^{2+}$ loading efficiency of DMN.

To compare the effect of those different modifications, the change in $\left[\mathrm{Ca}^{2+}\right]_{i}$ within the first $100 \mathrm{~ms}$ after stimulation (Fig. 4, dashed linear regressions on $\left[\mathrm{Ca}^{2+}\right]_{i}$ traces at $\mathrm{t}=0-100 \mathrm{~ms}$ ) was normalized to $\left[\mathrm{Ca}^{2+}\right]_{i}$ at stimulation, and then plotted against the logarithmic stimulation strength (Fig. 5). Under initial conditions (Fig. 5A, grey symbols), $\mathrm{Ca}^{2+}$ uncaging was done with $1.5 \mathrm{mM}$ intracellular $\mathrm{DMN}$ concentration, $2 \mathrm{mM}\left[\mathrm{Ca}^{2+}\right]_{0}$, and a dichroic mirror reflecting $15 \%$ of monochromator light and transmitting $85 \%$ of flash light to the objective.

The relative change in $\left[\mathrm{Ca}^{2+}\right]_{i}$ within $100 \mathrm{~ms}$ after onset of the $\mathrm{Ca}^{2+}$ uncaging stimulus scattered between $-69 \%$ and $+41 \%$, with an average of $-15.3 \% \pm 14.5 \%$ (mean \pm s.d.) in a range of $10 \mu \mathrm{M}$ to $15 \mu \mathrm{M}\left[\mathrm{Ca}^{2+}\right]_{i}$ (Fig $5 \mathrm{~F}$, grey error bars). A linear regression to the relative change was made (Fig. $5 \mathrm{~A}$, dotted grey line; slope of $0.82 \%$ per $\mu \mathrm{M}$ ), and it can be seen that at high $\left[\mathrm{Ca}^{2+}\right]_{i}$ the average decay became smaller. To check if there is a correlation between the relative $\left[\mathrm{Ca}^{2+}\right]_{i}$ change and the estimated kinetic type of increase in cumulative release (chapter 2.4.2), the data points were marked correspondingly (Fig. 5B, same data as in 5A). An apparent tendency of the relative change to be bigger at lower $\left[\mathrm{Ca}^{2+}\right]_{i}$ did not correlate with the appearance of mono-exponential rises in cumulative release time courses, but rather was a coincidence. An artifact by a stronger decay in $\left[\mathrm{Ca}^{2+}\right]_{i}$ would be reflected in temporal heterogeneity in release probabilities, being opposite to the observed, rather coincidental correlation. For the most common kinetic type, 2-exponential + line, examples with decaying, constant, and increasing $\left[\mathrm{Ca}^{2+}\right]_{i}$ could be found. 

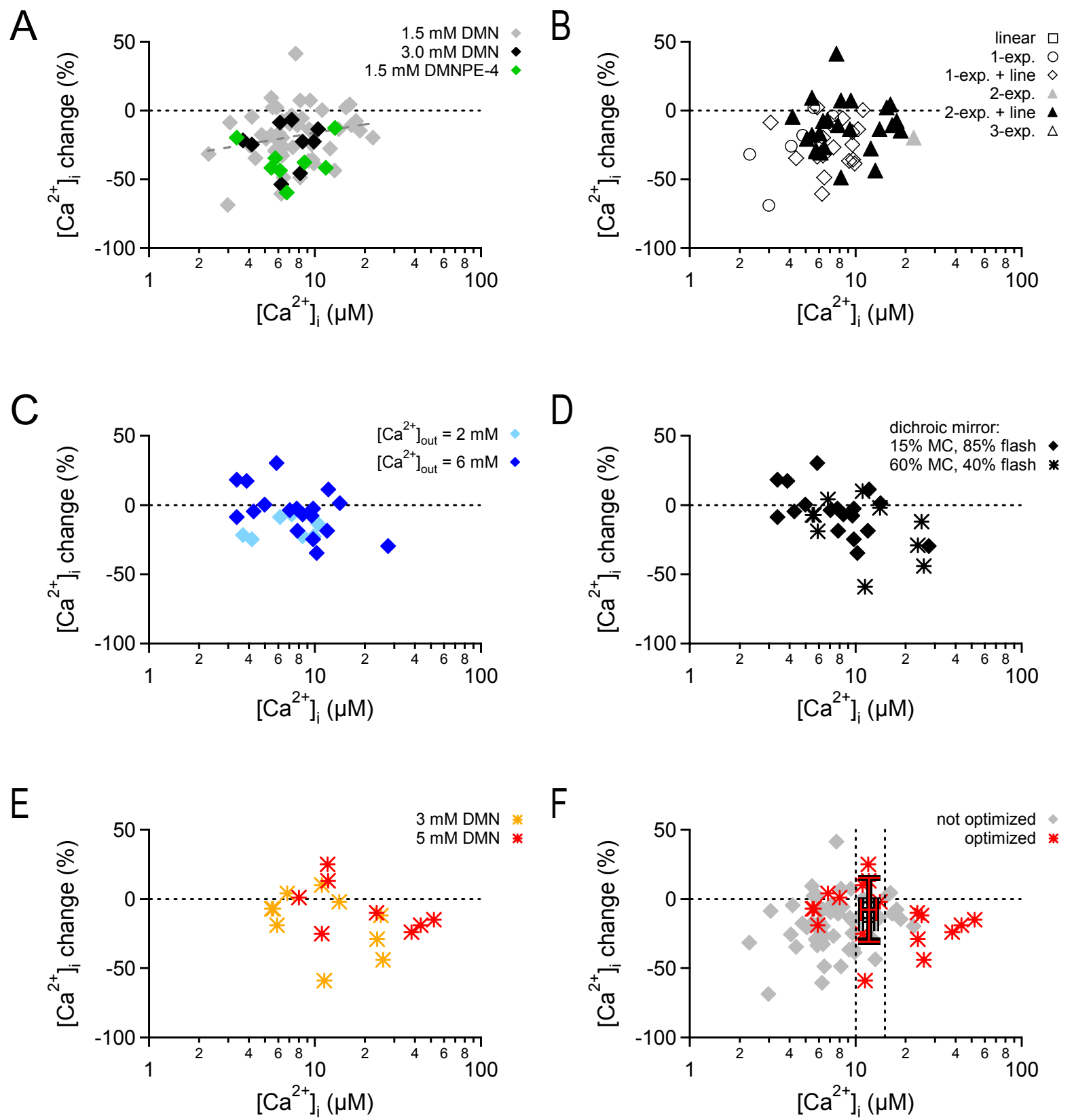

Fig. 5 Temporal $\left[\mathrm{Ca}^{2+}\right]_{i}$ changes in the presynaptic terminal after $\mathrm{Ca}^{2+}$ uncaging stimuli The $\left[\mathrm{Ca}^{2+}\right]_{i}$ change within $100 \mathrm{~ms}$ after onset of stimulation is plotted as a function of the initial stimulus intensity. Experimental conditions started with $1.5 \mathrm{mM}[\mathrm{DMN}], 2 \mathrm{mM}\left[\mathrm{Ca}^{2+}\right]_{0}, 15 \%$ of monochromator light and $85 \%$ of flash light (A, grey diamonds), and the corresponding data is separated into the classified kinetic types of release, as well (B). Successively and cumulatively, conditions were changed, as indicated (A, C - E), and finally, results after all optimization steps ( $\mathbf{F}$, red asterisks) were compared to conditions at the beginning ( $\mathbf{F} \& \mathbf{A}$, grey diamonds). At $10-15 \mu \mathrm{M}\left[\mathrm{Ca}^{2+}\right]$, averages were $-7.8 \% \pm 23.1 \%$ after optimization, and $15.3 \% \pm 14.5 \%$ before ( $F$, red and grey error bars, respectively). 
Since excitation light of $350 \mathrm{~nm}$ and $380 \mathrm{~nm}$ wavelengths has been used for $\left[\mathrm{Ca}^{2+}\right]_{\mathrm{i}}$ measurements, and since this should lead to photolysis of DMN as well, especially at $350 \mathrm{~nm}$ wavelength (Kaplan and Ellis-Davies, 1988), reduction of the $\mathrm{Ca}^{2+}$ decay by increasing the efficiency of ongoing $\mathrm{Ca}^{2+}$ uncaging due to the $350 \mathrm{~nm}$ monochromator light was aimed. Increasing the DMN concentration, and using lower flashlight intensities should result in comparable initial $\left[\mathrm{Ca}^{2+}\right]_{\mathrm{i}}$ increases, but thereafter, more DMN should remain for further uncaging by $350 \mathrm{~nm}$ monochromator light, counteracting the $\left[\mathrm{Ca}^{2+}\right]_{\mathrm{i}}$ decay. However, the data scattered in the same range as for the initial conditions (Fig. 5A, black and grey symbols). Moreover, changing the $\mathrm{Ca}^{2+}$ chelator to DMNPE-4 (kindly provided by GCR Ellis-Davies, Drexel university, Philadelphia PA, USA), which, in contrast to DMN, does not bind $\mathrm{Mg}^{2+}$, rather increased the relative decay (Fig 5A; green symbols).

A different strategy to reduce $\mathrm{Ca}^{2+}$ extrusion from the cytosol was the reduction of the $\mathrm{Ca}^{2+}$ extrusion driving force which plays an important role for $\mathrm{Na}^{+}-\mathrm{Ca}^{2+}$ exchangers (for reviews, see Guerini, 1998; Philipson and Nicoll, 2000). Therefore, the extracellular $\mathrm{Ca}^{2+}$ concentration $\left(\left[\mathrm{Ca}^{2+}\right]_{0}\right)$ has been raised from $2 \mathrm{mM}$ to $6 \mathrm{mM}$ (Fig. $5 \mathrm{C}$, light and dark blue symbols), while the DMN concentration was always $3 \mathrm{mM}$. Additionally, the imaging protocol (see above) after the flash stimulus has been optimized. The light absorbance of DMN is higher at $350 \mathrm{~nm}$ than at $380 \mathrm{~nm}$ (Kaplan and Ellis-Davies, 1988), so the imaging protocol directly after the flash-stimulus with three images at $380 \mathrm{~nm}$ and five subsequent image pairs at $380 \mathrm{~nm}$ and $350 \mathrm{~nm}$ was changed to ten image pairs without an initial $380 \mathrm{~nm}$ sequence (Fig. 4, black and blue traces, respectively). In cases with $6 \mathrm{mM}\left[\mathrm{Ca}^{2+}\right]_{0}$ and the new imaging protocol (Fig. 5C, dark blue symbols), the decay appeared to be smaller compared to the previous settings (light blue symbols), now showing many examples even with rising $\left[\mathrm{Ca}^{2+}\right]_{i}$. Based on this improvement, the monochromator light intensity has been increased additionally. The dichroic mirror joining light from the flash lamp and the monochromator was exchanged, now reflecting $60 \%$ monochromator light to the objective instead of previously $15 \%$, and as a consequence, the transmitted flash light was reduced from $85 \%$ to $40 \%$ (Fig. $5 \mathrm{D}$, diamonds and starts, respectively). However, in the range of $5 \mu \mathrm{M}$ to $12 \mu \mathrm{M}\left[\mathrm{Ca}^{2+}\right]_{\text {, }}$, an improvement did not seem to occur.

To further increase the $\mathrm{Ca}^{2+}$ uncaging efficiency by the monochromator, the DMN concentration has been raised from $3 \mathrm{mM}$ to $5 \mathrm{mM}$, keeping all other settings for optimization as before (Fig. 5E, orange and red symbols, respectively). A slight improvement might have occurred, but for a clearer result, the data with all optimization attempts $\left(6 \mathrm{mM}\left[\mathrm{Ca}^{2+}\right]_{0}, 60 \%\right.$ monochromator light, and $3 \mu \mathrm{M}$ or $5 \mu \mathrm{M}$ DMN concentration) were compared to the initial settings (2 mM $\left[\mathrm{Ca}^{2+}\right]_{0}, 15 \%$ monochromator light, and $1.5 \mu \mathrm{M} \mathrm{DMN}$; Fig. 5F). The averaged 
relative change in $\left[\mathrm{Ca}^{2+}\right]_{i}$ in a range of $10 \mu \mathrm{M}$ to $15 \mu \mathrm{M}\left[\mathrm{Ca}^{2+}\right]_{i}$ was $-15.3 \% \pm 14.5 \%$ for initial, and $-7.8 \% \pm 23.1 \%$ for improved conditions (mean \pm s.d.; Fig. 5F, grey, and red error bars, respectively). Unfortunately, a significant improvement could not be yielded ( $p=0.48$, twotailed, equal variance Student's T-Test). So all data, irrespective of their relative $\left[\mathrm{Ca}^{2+}\right]_{\mathrm{i}}$ change, was pooled and used for further analysis.

If not stated differently, all averages are given in mean \pm standard deviation, and significances were tested with student's T-tests (2 sample, unequal variance).

\subsubsection{Spatial $\left[\mathrm{Ca}^{2+}\right]_{\mathrm{i}}$ homogeneity after $\mathrm{Ca}^{2+}$ uncaging}

Spatial differences in $\left[\mathrm{Ca}^{2+}\right]_{i}$ should lead to different release probabilities for intrinsically identical vesicles, being identical also regarding their $\mathrm{Ca}^{2+}$ sensors. This effect might occur under a spatially inhomogeneous $\mathrm{Ca}^{2+}$ uncaging efficiency. When spatial differences in $\left[\mathrm{Ca}^{2+}\right]_{i}$ lead to spatial differences in release probabilities, and when these release probabilities are erroneously correlated with the measured, globally averaged $\left[\mathrm{Ca}^{2+}\right]_{\mathrm{i}}$, the results could be mistaken as different pools of vesicles with intrinsically different $\mathrm{Ca}^{2+}$ sensitivities.

\section{Spatial $\left[\mathrm{Ca}^{2+}\right]_{i}$ homogeneity in the $x-y$ plane}

To rule out such effects, spatial homogeneity in $\left[\mathrm{Ca}^{2+}\right]_{i}$ has been tested. First, the horizontal plane, i.e. the x-y plane of the fluorescence images, was examined (Fig. 6A). Six pixels in the area of the presynaptic terminal were chosen such that several locations along the terminal, with different distances to the patch pipette, could be compared (Fig. 6A, red pixels in fluorescence images). As can be seen for three different example cells (Fig. 6A1 - A3), the patch pipette was placed to the left of the presynapse, and intracellular fluorescence intensities were maximal close to the tip of the pipette. The pixels in each cell were numbered in increasing order from left to right, and the relative difference in $\left[\mathrm{Ca}^{2+}\right]_{i}$ for each pixel was plotted (Fig. 6A, left panels). These relative differences were calculated by normalizing the individual $\left[\mathrm{Ca}^{2+}\right]_{i}$ to the spatial average at a given stimulus. In each cell, five $\mathrm{Ca}^{2+}$ uncaging stimuli were applied (Fig. 6A, left panels, flashes marked with different symbols). For the first example cell shown (Fig. 6A1), the spatial differences in $\left[\mathrm{Ca}^{2+}\right]_{i}$ ranged between $\pm 11 \%$, and the largest difference with totally $22 \%$ was observed at strongest stimulation to $16 \mu \mathrm{M}\left[\mathrm{Ca}^{2+}\right]_{\mathrm{i}}$ (Fig. $6 \mathrm{~A} 1$, open squares; $13-15 \%$ difference at $5-6 \mu \mathrm{M}$ for other stimuli). In the second cell (Fig. 6A2), differences were slightly bigger, ranging between 
$\pm 14 \%$, and being maximal with $25 \%$ at $9 \mu \mathrm{M}\left[\mathrm{Ca}^{2+}\right]_{\mathrm{i}}$ (closed triangles; $13 \%$ difference at 38 $\mu \mathrm{M}$, open diamonds; $19-21 \%$ at $7-11 \mu \mathrm{M}$ for other stimuli). In the third cell tested (Fig. 6A3), spatial differences for five pixels examined were bigger; in a detected range of - $17 \%$ to $+22 \%$, a maximal difference of $39 \%$ at $10 \mu \mathrm{M}\left[\mathrm{Ca}^{2+}\right]_{\mathrm{i}}$ showed up (open circles; $23 \%$ difference at $31 \mu \mathrm{M}$, asterisks; $22-30 \%$ at $6-12 \mu \mathrm{M}$ for other stimuli).

Thus, spatial inhomogeneity in $\left[\mathrm{Ca}^{2+}\right]_{\mathrm{i}}$ was apparent. However, the spatial imaging resolution of the system with a pixel length of $1.48 \mu \mathrm{m}$ (at 8 * 15 binning) was low, and furthermore, $\left[\mathrm{Ca}^{2+}\right]_{\mathrm{i}}$ estimates from single pixels were expected to scatter due to measurement noise. Thus, here a distinction between real spatial differences in $\left[\mathrm{Ca}^{2+}\right]_{i}$ and scatter due to measurement noise could not be made. Real spatial inhomogeneity in $\left[\mathrm{Ca}^{2+}\right]_{\mathrm{i}}$ might have been due to diffusional barriers such as fingerlike structures with high access resistances in the calyx of Held (Wimmer et al., 2004), or locally concentrated $\mathrm{Ca}^{2+}$ pumps or $\mathrm{Ca}^{2+}$ exchangers (Guerini, 1998; Philipson and Nicoll, 2000). However, thin, fingerlike structures at calyces of Held in rats were rather visible at an age of 14 days, as compared to 8 days old rats (Wimmer et al., 2004), and measurements in this study were performed using P8 - P10 rats. Moreover, $\mathrm{Ca}^{2+}$ uncaging stimuli were separated by at least 2 minutes, so that diffusional exchange of $\mathrm{Ca}^{2+}$ chelator molecules across the whole terminal was likely.

Fig. 6 Spatial $\left[\mathrm{Ca}^{2+}\right]_{i}$ homogeneity in the presynaptic terminal after $\mathrm{Ca}^{2+}$ uncaging A Comparison of $\left[\mathrm{Ca}^{2+}\right]_{i}$ at different spots within the horizontal $x-y$ plane of the presynaptic terminal. Differences in the initial $\left[\mathrm{Ca}^{2+}\right]_{i}$ after $\mathrm{Ca}^{2+}$ uncaging are plotted against different locations in the terminal (A, left panels). Five different stimuli were applied within the same cell (different symbols in left panels), and three different cells were compared (A1 - A3, respectively). In the fluorescent images (A, right panels), spots are specified with the corresponding pixel number from left to right (right panels, pink shaded pixels; left panels, abscissa). For cell A3, the $6^{\text {th }}$ pixel was placed at the axon. B Efficiency of UV light illumination in the vertical direction ( $\mathrm{z}$ axis). B1 Illustration of $\mathrm{Ca}^{2+}$ uncaging in the calyx of Held (thick black line), being inhomogeneous in the z-direction. The focal plane for $\mathrm{Ca}^{2+}$ uncaging appears as a red dashed line, $\left[\mathrm{Ca}^{2+}\right]_{i}$ is symbolized by different shades of red, vesicles appear as circles, and arrow sizes represent different release probabilities. B2 Dextran-bound DMNB-FITC was immobilized in a gel, and FITC was uncaged with a UV flash. An x-z image of FITC fluorescence intensities is shown, and intensities are represented by different shades of grey. Brightness was adjusted to $40-100 \%$ of the total scale. B3 Limits are shown, where the fluorescence intensity in the $x-z$ plane (as shown in B2) dropped by more than $0.1 \%, 5 \%$, and $10 \%$ (blue lines from center to periphery, respectively), as normalized to the brightest spot in the $x-y-z$ center. The red line marks the position of maximal fluorescence in each $x$-axis linescan, and compares them for different z-positions. 
$\mathrm{A}_{1}$
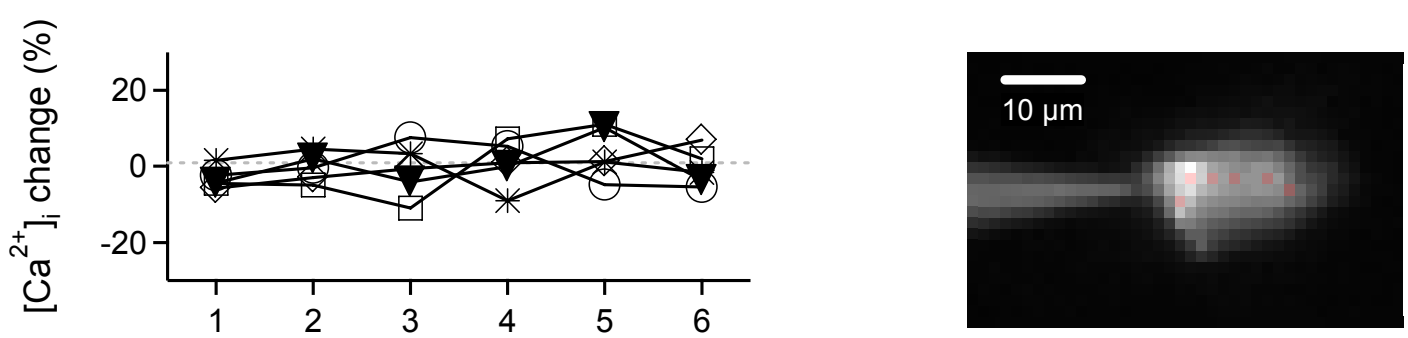

$\mathrm{A} 2$
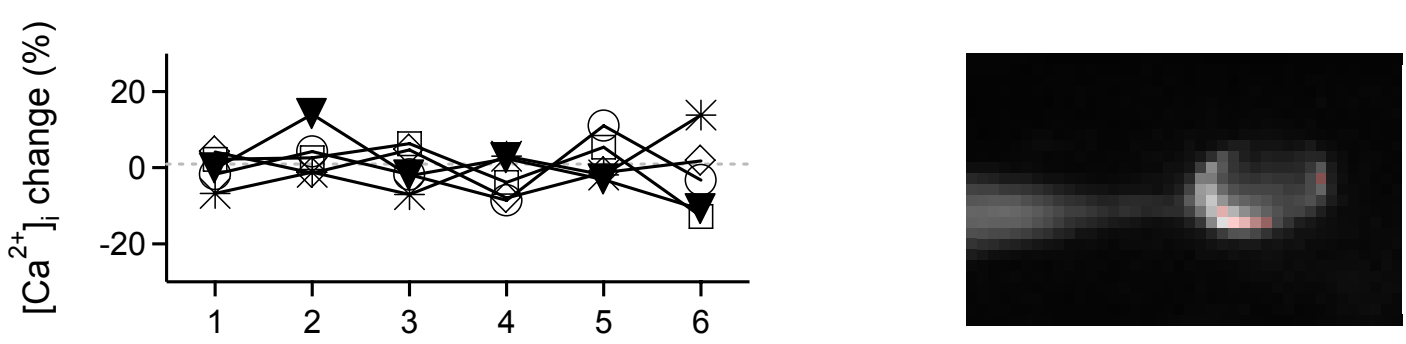

A3
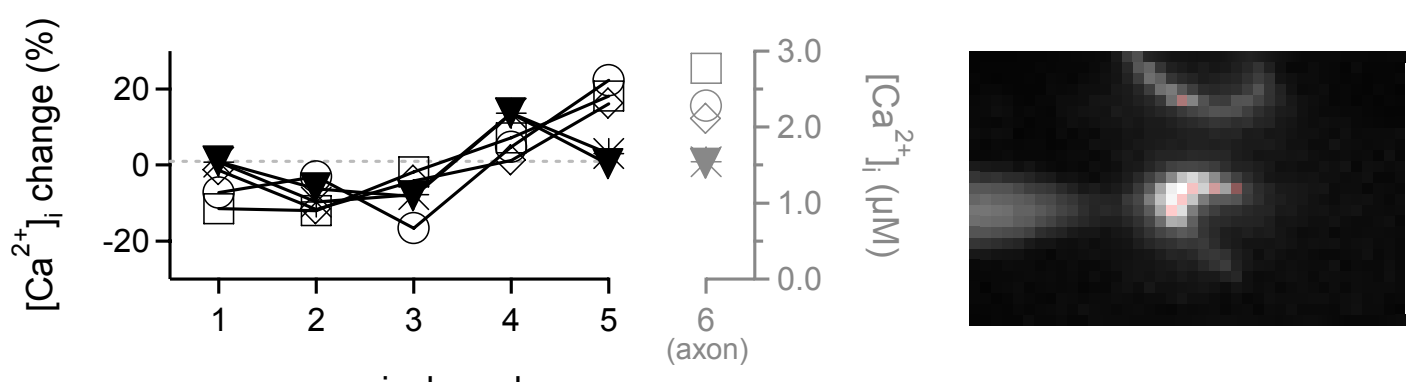

pixel number

in each cell $5 \mathrm{Ca}^{2+}$ uncaging stimuli have been applied:

\begin{tabular}{|c|c|c|c|c|c|}
\hline \multirow[b]{2}{*}{$\square$} & \multirow{2}{*}{$\begin{array}{l}\text { flash } \\
\# 1\end{array}$} & \multicolumn{4}{|c|}{$\left[\mathrm{Ca}^{2+}\right]_{\mathrm{i} \text {, avg }}$ and normalized maximal spatial differences } \\
\hline & & $16 \mu \mathrm{M}, \quad 22 \%$ & $11 \mu \mathrm{M}, \quad 19 \%$ & $6 \mu \mathrm{M}$ & $30 \%$ \\
\hline$\theta$ & \# 2 & $5 \mu \mathrm{M}, \quad 13 \%$ & $7 \mu \mathrm{M}, \quad 20 \%$ & $10 \mu \mathrm{M}$ & $39 \%$ \\
\hline$\rightarrow$ & \#3 & $5 \mu \mathrm{M}, \quad 15 \%$ & $9 \mu \mathrm{M}, \quad 25 \%$ & $12 \mu \mathrm{M}$ & $22 \%$ \\
\hline * & \# 4 & $6 \mu \mathrm{M}, \quad 14 \%$ & $9 \mu \mathrm{M}, \quad 21 \%$ & $31 \mu \mathrm{M}$ & $23 \%$ \\
\hline 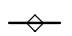 & \# 5 & $6 \mu \mathrm{M}, \quad 13 \%$ & $38 \mu \mathrm{M}, \quad 13 \%$ & $8 \mu \mathrm{M}$ & $28 \%$ \\
\hline
\end{tabular}

$\mathrm{B}_{1}$

$\mathrm{B} 2$
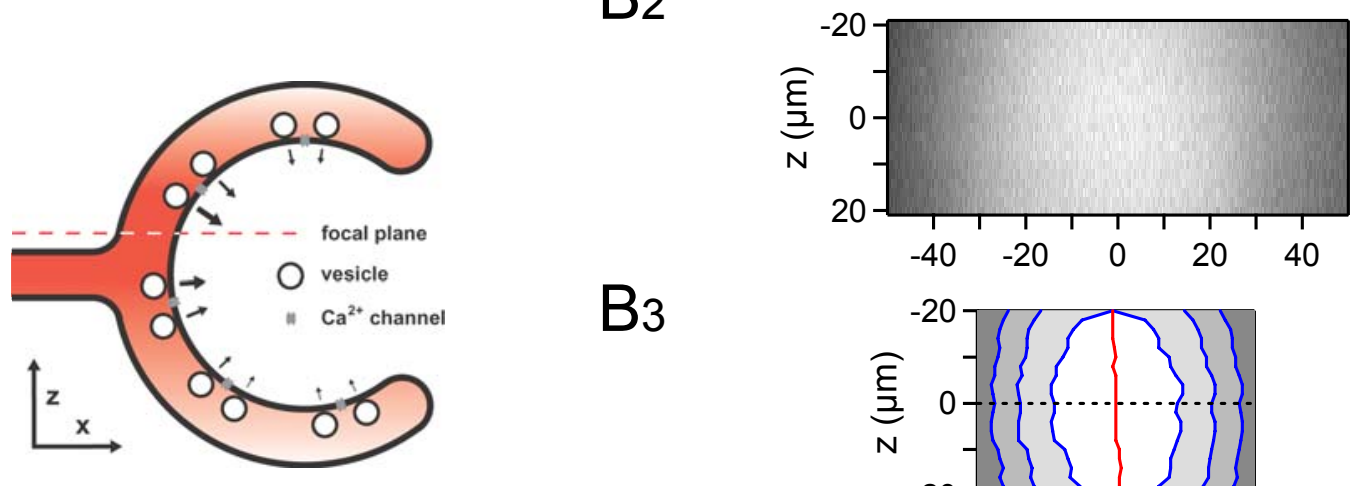

B3

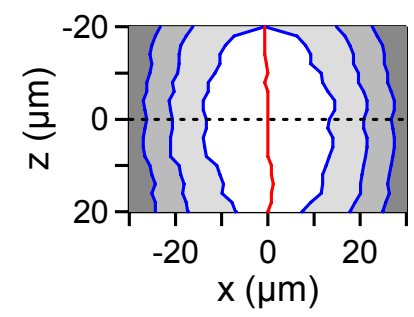


An effect on spatial $\left[\mathrm{Ca}^{2+}\right]_{i}$ by $\mathrm{Ca}^{2+}$ pumps and exchangers was expected to become visible when using the $\mathrm{Ca}^{2+}$ chelator DMNPE-4 (e.g. in Fig. 5A, green symbols), instead of DMN. DMNPE-4 does not bind $\mathrm{Mg}^{2+}$, and therefore inhomogeneous $\mathrm{Ca}^{2+}$ occupancy of the $\mathrm{Ca}^{2+}$ chelator due to increased $\mathrm{Mg}^{2+}$ binding close to $\left[\mathrm{Ca}^{2+}\right]_{\mathrm{i}}$ sinks at $\mathrm{Ca}^{2+}$ pumps and exchangers could be ruled out, using DMNPE-4. These control experiments reproduced results from experiments with DMN convincingly, also eliciting biphasic release kinetics with comparable release kinetics (not shown). But still, spatial $\left[\mathrm{Ca}^{2+}\right]_{i}$ inhomogeneity could not be ruled out, but was assumed not to play a crucial role for the appearance of two kinetically distinct components of release.

Also, the $\mathrm{Ca}^{2+}$ uncaging efficiency very far away from the patch-pipette has been investigated by choosing a pixel sitting on the axon of the same cell (Fig. 6A3, pixel 6). The axon was identified by taking a high resolution image stack with vertical steps of $1 \mu \mathrm{m}$, and tracing the axon back starting at the identified terminal (not shown). Note, that the axon lay outside the area of flashlight illumination (see above), thus not being subject to photostimulation. Here, the different $\mathrm{Ca}^{2+}$ uncaging stimuli could not efficiently increase the intra-axonal $\mathrm{Ca}^{2+}$ concentration, even not when $\left[\mathrm{Ca}^{2+}\right]_{\mathrm{i}}$ in the terminal was increased to more than $30 \mu \mathrm{M}(\mathrm{Fig}$. $6 \mathrm{~A} 3$, asterisks).

\section{Spatial $\left[\mathrm{Ca}^{2+}\right]_{i}$ homogeneity in the vertical direction}

When an epi-fluorescence microscope is used, as done in this study, excitation light passes through the objective to the specimen, and therefore the light concentrates in the focal plane. With increasing distance to the focal plane (illustrated for a calyx of Held in Fig. 6B1, dashed red line represents focal plane), the illumination intensity will decrease, and the $\mathrm{Ca}^{2+}$ uncaging efficiency will be attenuated (Fig. $6 \mathrm{~B},\left[\mathrm{Ca}^{2+}\right]_{i}$ symbolized by shades of red). This effect is of advantage for experimental strategies that aim at locally restricted excitation by focused light (reviewed in Denk et al., 1994; Katz and Dalva, 1994). But in this study, homogeneous $\mathrm{Ca}^{2+}$ uncaging efficiencies are important, not to artificially induce inhomogeneous release probabilities within a single presynaptic terminal (Fig. 6B1, arrows). Thus, out-of-focus attenuation of UV light intensity should be small. A large illumination window, as used here $(30 * 30 \mu \mathrm{m}$, see above), should decrease out-of-focus attenuation, but still, attenuation might be effective due to filtering by tissue and introduced chemicals. It was shown that $\mathrm{Ca}^{2+}$ chelators themselves filter excitation light used for photolysis (EllisDavis, 2003).

Here, an indirect approach to measure $\mathrm{Ca}^{2+}$ uncaging homogeneity in the vertical direction was made. The fluorescent dye FITC in a DMNB-caged and dextran-bound form $(0.2 \mathrm{mM}$; 
MW $10000 \mathrm{~g} / \mathrm{mol}$; Molecular probes, OR, USA) was immobilized in a gelatine block ("Sofort Gelatine, weiß, kalte Zubereitung", $0.2 \mathrm{~g} / \mathrm{ml}$; Bella Back, Germany). Spectral analysis of light transmission through the gelatine confirmed suitability of the gelatine for this approach (Genesys 5 spectrophotometer, Spectronic Instruments, Germany; spectrogram not shown). After FITC uncaging in the gelatine block, fluorescence images in the horizontal plane $(x-y)$ were taken at different vertical positions $(z)$, using a piezoelectric objective-positioner (PI-Foc, Physics Instruments, Germany; controlled by TILLvisION software; $\Delta z=2 \mu \mathrm{m})$. In fluorescence images acquired, line scans were taken in the $\mathrm{x}$-direction through the center of illumination, with y always equaling $0 \mu \mathrm{m}$., and fluorescence intensities were plotted against the z-position (Fig. 6B2). The center of illumination in the focal plane was located at $0 \mu \mathrm{m}$ for $\mathrm{x}, \mathrm{y}$, and z. Relative brightness was adjusted (40\%-100\% of maximal), so that decreases in fluorescence intensity became more apparent in this display. Decreases in fluorescence intensity were analyzed, and regions in which the fluorescence intensity dropped by more than $0.1 \%, 5 \%$, or $10 \%$ as compared to the $x-y-z$ center of illumination are shown in an $x-z$ plot (Fig. 6B3, limits indicated by blue lines and shades of grey, respectively). The maximal fluorescence for each $x$-direction line-scan was read out as well (red line), and was detected at comparable $x$-positions throughout the whole z-range scanned. Thus, for different $z$ positions, the center of illumination did not shift in the x-direction. Furthermore, within a volume of about $40 * 40 * 40 \mu \mathrm{m}^{3}$, uncaging efficiency did not decrease by more than $5 \%$ (Fig. 6B3, second blue lines from center). Calyces of Held have a diameter of typically $10-$ $20 \mu \mathrm{m}$ (for P8 - P10 old rats), and therefore illumination should be homogeneous enough not to artificially elicit inhomogeneous release probabilities. Filter effects by DMNB, as used here, and by DMN, as used for $\mathrm{Ca}^{2+}$ uncaging, should be comparable, and therefore a marked contribution was ruled out. However, the homogeneous UV illumination in the z-direction also implies a relatively low restriction of FITC excitation and FITC fluorescence detection in the z-direction. Thus, out-of-focus fluorescence contributed to fluorescence intensities at each zposition, and the results were blurred accordingly. Moreover, the gelatine was expected to have different spectral properties than tissue slices and chemicals applied in experiments, and therefore, illumination inhomogeneities in experiments could not be fully excluded, although being unlikely.

Control experiments might be improved using confocal microscopy for fluorescence detection in the gel block (Denk et al., 1994; Katz and Dalva, 1994), so that blurring effects due to outof-focus illumination is minimized. However, for a confocal approach, shortcomings of limited scan-ranges in the z-direction, and of necessary cover slips, which usually filter UV light, must be avoided. 


\subsection{Electrophysiological recordings}

\subsubsection{Deconvolution of postsynaptic recordings}

To calculate the time course of presynaptic quantal release, the technique of EPSC deconvolution was used (Igor Pro macro by Erwin Neher; Version April $4^{\text {th }}, 2002$; downloadable at http://www.mpibpc.gwdg.de/abteilungen/140/software/index.html), as described by Neher and Sakaba (Neher and Sakaba, 2001; Sakaba and Neher, 2001a). Fusion of a single presynaptic vesicle leads to a small postsynaptic current (PSC), called the miniature postsynaptic current (mPSC). Under resting conditions with low presynaptic release rates, single mPSCs can easily be detected, but when the release rate increases, e.g. due to stimulation, at many synapses, like the calyx of Held, mPSCs sum up to large PSCs, making a direct count of quanta released impossible. Therefore, the EPSC deconvolution method uses the amplitude and time course of mPSCs to deconvolve the larger PSCs, and to convert them into presynaptic release rates as a function of time (Cohen et al., 1981; van der Kloot, 1988; Aumann and Parnas, 1991). Meanwhile, this deconvolution method has been extended to take glutamate spillover currents into account (Neher and Sakaba, 2001; Sakaba and Neher, 2001a): The delayed clearance of transmitter molecules from the synaptic cleft (Barbour et al., 1994; Mennerick and Zorumski, 1995), resulting in a residual postsynaptic current, has been incorporated into the approach (Neher and Sakaba, 2001), so that this optimized deconvolution method became applicable for excitatory glutamatergic synapses such as the calyx of Held. For better understanding, residual currents, as returned by the deconvolution routine, were displayed additionally to the corresponding EPSCs in some cases.

To gain reasonable deconvolution results, the parameters for the algorithms have to be determined individually for each cell. Thus, a fitting protocol to extract the wanted parameters (Neher and Sakaba, 2001) was given for each pre- and postsynaptic pair of cells patched (e.g. in Fig. 7, same cell as Fig. 21). The fitting protocol was composed of five different depolarization steps from $+80 \mathrm{mV}$ to $0 \mathrm{mV}$ for varying periods $(0.8-10 \mathrm{~ms}$; Fig. 7 , uppermost trace), and voltage steps started at, and went back to $+80 \mathrm{mV}$ to increase the efficiency of $\mathrm{Ca}^{2+}$ influx (Fig. 7, brown trace). The second to fifth step had durations of 2, 4, 5, and $10 \mathrm{~ms}$, respectively. The first step typically lasted $0.8 \mathrm{~ms}$, but was time-adjusted in each cell to get a corresponding EPSC amplitude of about $1 \mathrm{nA}$ (Fig. 7, red trace). At this small amplitude, EPSC decay time courses were assumed to be similar to mEPSC kinetics, because only at few active zones release should have taken place, and in a single active 

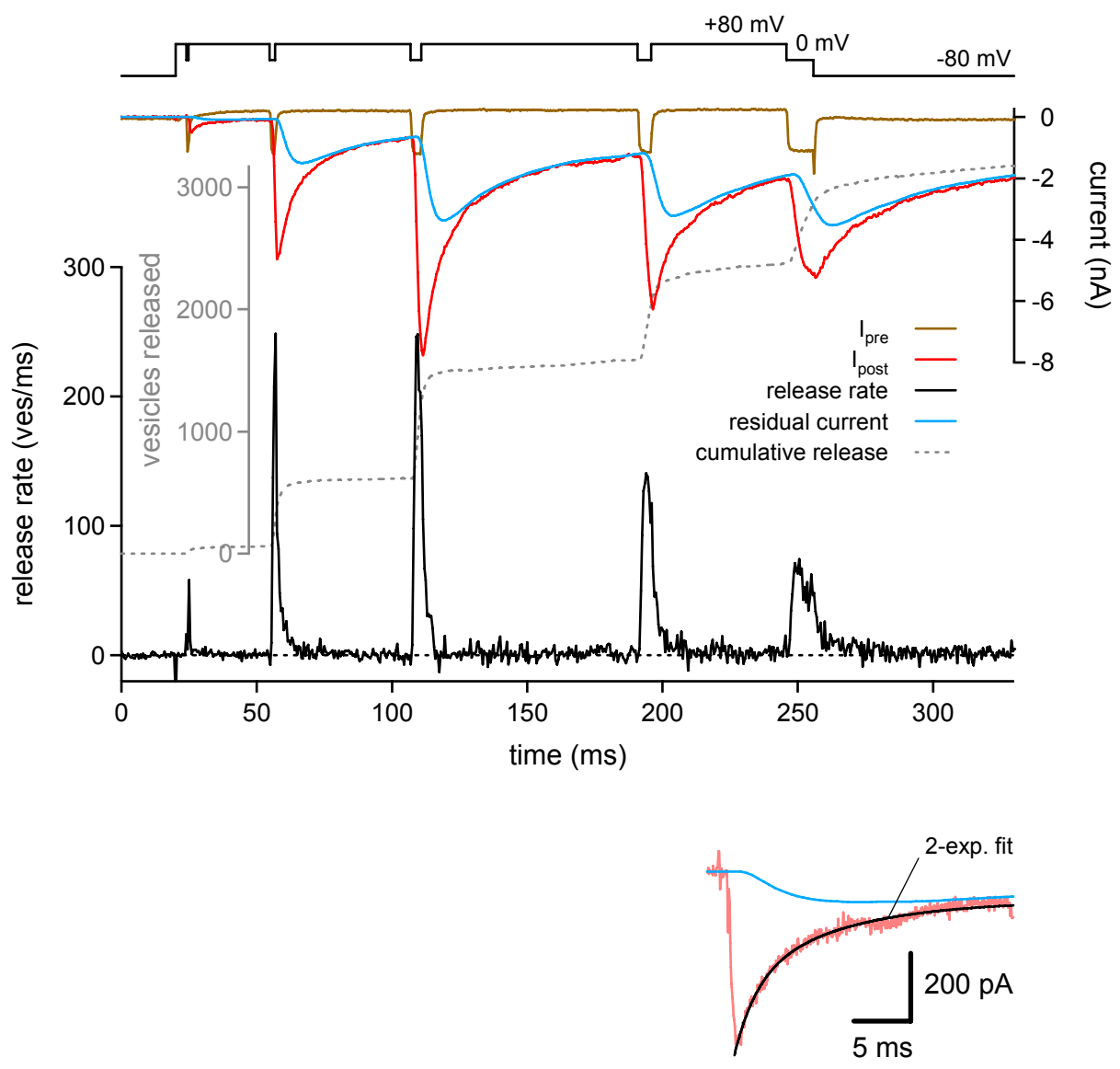

Fig. 7 Stimulus protocol to approximate EPSC deconvolution parameters

Depolarizing steps from a holding potential of $+80 \mathrm{mV}$ to $0 \mathrm{mV}$ were applied, and stimulus lengths were varied $\left(0.8,2,4,5\right.$, and $10 \mathrm{~ms}$; uppermost trace). Presynaptic $\mathrm{Ca}^{2+}$ influx (brown trace) induced EPSCs (red trace), and the response to the first, small stimulus has been fitted with a 2-exponential function (magnified view below, black curve) to estimate mEPSC decay parameters. Deconvolution yielded an estimate for residual current and release rates (blue and black traces, respectively), and the time course of cumulative release (dashed grey trace) was calculated.

zone, only a single vesicle should have fused, so that quantal glutamate spillover at a postsynaptic receptor population was not likely to take place.

The decay of this small EPSC then was fitted with a mono-exponential, and additionally with a double-exponential function to approximate the decay time course of a mEPSC for the cell tested. In most cases, the decay was biphasic (Fig. 7, inset), and the time constants could be used to optimize the deconvolution parameters. Fast and slow decay typically had time constants of about $1-3$ and $10-15 \mathrm{~ms}$, respectively, comparing well to previous findings under CTZ application (Schneggenburger and Neher, 2000). The mini amplitude $q$ was set to -30 pA (Neher and Sakaba, 2001), or to -15 pA for experiments with $2 \mathrm{mM} \gamma$-DGG. Additional free parameters for the deconvolution were the exponent of the power law of glutamate 
channel activation $n$, the exponent of the diffusion law $n_{D}$, the synaptic cleft diffusion distance $r_{d}$, the fraction of the slow component $\alpha$, and the residual current weighting factor $\beta$ (Neher and Sakaba, 2001). By this means, the time courses of the presynaptic release rate (Fig. 7, black trace) and cumulative release (dashed grey trace) could be calculated, taking the estimated residual current (blue trace) into account. Typically, the parameters reached values of: $n=1.18, n_{D}=0.7, r_{d}=0.9, \alpha=0.2, \beta=80$.

Since the fitting protocol should cover the whole range of experimental stimulation strengths, stimuli weaker or stronger than those applied during the fitting protocol, but being deconvolved with the same parameters, were further analyzed under the assumption that the set of parameters can be linearly extrapolated beyond the range of stimuli tested. This was relevant for some strong $\mathrm{Ca}^{2+}$ uncaging stimuli. Here, for a better approximation of deconvolution parameters, it was taken care that late release did not reach negative values, because negative release indicates wrong adjustment of deconvolution parameters (note, that endocytosis does not appear in EPSCs). Typically, $n$ had to be increased by $10-20 \%$, or $\beta$ decreased by $20-30 \%$, and often $n_{D}$ had to be readjusted then as well.

Finally, the presynaptic release rate as a function of time, or the cumulative release by integrating the release rate, resulted from the deconvolution approach.

\subsubsection{Fitting the cumulative release time courses}

Time courses of cumulative release were fitted with several algorithms to extract components hidden in the compound release trace. It can be assumed, that releases from the readilyreleasable pool (RRP) in response to homogeneous and continuous stimulation, or better in response to a uniform and constant release probability, follows an exponential until the pool is depleted. $\mathrm{A} \mathrm{Ca}^{2+}$ uncaging stimulus should meet these stimulation criteria (Wölfel et al., 2003), but also responses to depolarizations could be fitted (Sakaba and Neher, 2001b) to get important information.

The applied fitting routines were based on 6 different algorithms (user-defined functions in Igor Pro), yielding the time constant and amplitude of release for each component that was assumed to exist by the routines. The fits were checked for validity, and a qualitative comparison was made to find the best description of each single cumulative release trace. For the algorithms used, $n_{\text {ves }}$ was the number of vesicles released at a given point of time $t$, $N_{\text {ves }}$ was the total number of vesicles releasable (pool size), and $\tau$ was the time constant of 
release. If more than one component of release with exponential kinetics was assumed, parameters of each component were indexed with increasing, stating with the component with the fastest release kinetics, then the second fastest, and so on. In some routines, an additional linear component was described by its slope $s_{\text {lin }}$.

Linear time course ('line):

$$
n_{\text {ves }}(t)=s_{\text {lin }} \cdot t
$$

Mono-exponential time course ('1-exp. ):

$$
n_{v e s}(t)=N_{v e s}-N_{v e s} \cdot \exp \left(\frac{-t}{\tau}\right)
$$

Mono-exponential time course with an additional linear component ('1-exp. + line):

$$
n_{\text {ves }}(t)=N_{\text {ves }}-N_{\text {ves }} \cdot \exp \left(\frac{-t}{\tau}\right)+s_{\text {lin }} \cdot t
$$

Double-exponential time course ('2-exp. ):

$$
n_{\text {ves }}(t)=N_{\text {ves }}-N_{1} \cdot \exp \left(\frac{-t}{\tau_{1}}\right)-N_{2} \cdot \exp \left(\frac{-t}{\tau_{2}}\right)
$$

Double -exponential time course with an additional linear component ('2-exp. + line):

$$
n_{\text {ves }}(t)=N_{\text {ves }}-N_{1} \cdot \exp \left(\frac{-t}{\tau_{1}}\right)-N_{2} \cdot \exp \left(\frac{-t}{\tau_{2}}\right)+s_{\text {lin }} \cdot t
$$

Triple-exponential time course ('3-exp. ):

$$
n_{\text {ves }}(t)=N_{\text {ves }}-N_{1} \cdot \exp \left(\frac{-t}{\tau_{1}}\right)-N_{2} \cdot \exp \left(\frac{-t}{\tau_{2}}\right)-N_{3} \cdot \exp \left(\frac{-t}{\tau_{3}}\right)
$$

Additionally to the amplitudes and time constants of release, the delay of release could be extracted as the back-extrapolated time point where the fit intercepted the abscissa, i.e. the level of no release before stimulation induced vesicle release.

In a few cases with low $\left[\mathrm{Ca}^{2+}\right]_{\mathrm{i}}$ after $\mathrm{Ca}^{2+}$ uncaging, cumulative release had an apparently linear time course, as would be expected because of very large release time constants. Here, 
the time constant of release $\tau$ was defined by the time, when the linear regression reached (1 $-1 / e) \approx 63 \%$ of the total pool size, taking the slope of the linear regression $s_{\text {lin }}$ into account:

$$
\tau=\frac{N_{v e s}}{S_{\text {lin }}} \cdot\left(1-\frac{1}{e}\right)
$$

The total pool size $N_{\text {ves }}$ could be estimated from release after a 50 ms depolarization usually following the uncaging stimulus.

Since most $\mathrm{Ca}^{2+}$ uncaging stimuli where followed by a $50 \mathrm{~ms}$ depolarization to completely deplete vesicles from the RRP, the fit range after the $\mathrm{Ca}^{2+}$ uncaging stimulus was limited by the onset of the succeeding depolarization. So in most cases, the fit range ended $100 \mathrm{~ms}$ after uncaging, and in some other cases $50 \mathrm{~ms}$ after uncaging. The start point of fitting was set to the time point when about $10 \%$ of total cumulative release was reached.

To prevent wrong estimates of timing and amplitudes, the cumulative release traces were $x-$, and y-shifted, before being fitted: the start point for fitting was time-shifted to meet zero, and the baseline of the cumulative release trace shortly before onset of stimulation was set to zero as well. After fitting, the original settings of the cumulative release trace were restored, and the fitted regressions were redefined extending to the whole time range needed for analysis, especially the delay estimates.

The quality of each fit was tested by several means. Beside release time constants, amplitudes, delays, and slopes, also the residual, i.e. the difference between the original release time course and the corresponding fit trace, was examined within the time range of fitting. The criteria for a valid fit were:

- Delay criterion: the delay of the response had to be a positive value (delay $>0$ ).

- Time constant criterion: for several exponential components, their time constants had to differ by a factor larger or equal three (e.g. $\tau_{1} \leq\left(\tau_{2} / 3\right)$ AND $\tau_{2} \leq\left(\tau_{3} / 3\right)$ ).

- Amplitude criterion: for several exponential components, the amplitude of each component had to contribute by at least $10 \%$ of total (e.g. $\left(N_{i} \geq 0.1 \cdot N_{1}+N_{2}+N_{3}\right)$ with $i=\{1 ; 2 ; 3\})$.

- Linear slope criterion: For regressions with a linear component, the slope of this component had to be positive ( $s_{\text {lin }}>0$ ves/ms).

- Peak difference criterion: the maximum of the residual (absolute values) had to be smaller than 100 vesicles $\left(\Delta n_{\max }<100\right.$ ves). 
- Integrated difference criterion: the integral of the residual (absolute values) had to be smaller than 2 vesicles within $100 \mathrm{~ms}\left(\int \Delta n<20\right.$ ves $\left.\mathrm{s}\right)$.

Among the fits accepted as valid, the rule of precedence was based on two postulates. First, a simple regression with few parameters should be preferred over more complicated approximations. Second, refilling of released vesicles is likely to take place, but within 100 ms fitting range only the observed fast refilling of slow vesicles (Sakaba and Neher, 2001b) might show up with a maximal rate of about $10 \mathrm{ves} / \mathrm{ms}$. Due to the relatively slow refilling rate, as compared to release from the RRP, refilling should show up in the cumulative release trace as a linear component. Thus, regressions including a linear component are preferred, especially to improve estimates for cases where the release rate is slow and close to a possible refilling rate.

The resulting rule of precedence was:

$$
\begin{array}{ll}
\text { 1. } & \text { 1-exponential + line } \\
\text { 2. } & \text { 2-exponential + line } \\
\text { 3. } & \text { 1-exponential } \\
\text { 4. } & \text { 2-exponential } \\
\text { 5. } & \text { 3-exponential }
\end{array}
$$

For a depolarizing $100 \mathrm{~Hz}$ train stimulus (Fig. 10A - C, black traces), cumulative release during the short interstimulus periods of $10 \mathrm{~ms}$ were analyzed in a different way. Here, fast release was estimated by the number of vesicles released during the first $2 \mathrm{~ms}$ after onset of each stimulus. Within this time, the release rate dropped from its fast initial time course to a second, slower component, as visible in the decay phase of the release rate. The amount of release during the remaining $8 \mathrm{~ms}$ yielded the corresponding estimate for the slow release component.

Peak release rates for the fast release component were extracted directly from the release rate traces, but peak release rates for the slow release component were quantified indirectly. After subtraction of the assumed fast release component from the cumulative release trace, the initial slope of remaining cumulative release has been fitted with a linear regression, typically in a range of $10 \mathrm{~ms}$ after the uncaging stimulus (e.g. in Fig. 12B - C, dashed green line). The approximated initial slope gave the indirect estimate of the peak release rate of the slow release component. 
If not stated differently, all averages are given in mean \pm standard deviation, and significances were tested with student's T-tests (2 sample, unequal variance).

\section{Variants in approximating refilling of the RRP}

When fitting to cumulative release traces, refilling of the RRP was taken into account by also using regressions that include an additional linear component ('1-exp. + line', and '2-exp. + line'; see above). To verify this approach, an alternative method was tested and compared, which subtracts a refilling component from the cumulative release trace (the macro for Igor Pro analysis was kindly provided by Prof. Dr. Erwin Neher), before further analysis was done. Here, the assumed speed of refilling depends on the fraction of pool depletion, thus, the fewer vesicles are left, the faster refilling becomes. The user empirically adjusts the refilling time constant and the time point $t_{\text {end }}$ at which the initially available pool of vesicles is assumed to be depleted, such that release ends at the given time point $t_{\text {end }}$ after subtraction of refilling. After subtracting refilling with this approach, further analysis was done by fitting different types of regressions as described before.

A direct comparison was made between the two approaches, i.e. fitting with exponential functions including a linear component on one hand, or initial refilling correction and subsequent fitting with exponential functions without a linear component on the other hand (Fig. 8). Three examples of cumulative release at different $\mathrm{Ca}^{2+}$ uncaging intensities of $7 \mu \mathrm{M}$, $11 \mu \mathrm{M}$, and $52 \mu \mathrm{M}\left[\mathrm{Ca}^{2+}\right]_{i}$ are shown without, and with initial refilling correction (Fig. 8, black and grey traces, respectively). The two lower stimulus responses were recorded in the same cell, and here, refilling correction had a bigger effect at the lowest stimulus strength. Applying the described fitting routines to the cumulative release traces (see above), resulted in time course estimates being '2-exponential + line' for all uncorrected traces (Fig. 8, dashed red lines), and being '2-exponential' for all refilling corrected traces (Fig. 8, dashed orange lines). To illustrate the qualitative differences between the different regression types fitted, the '2exponential' approximation to the uncorrected release trace at the highest stimulation strength of $52 \mu \mathrm{M}\left[\mathrm{Ca}^{2+}\right]_{i}$ is plotted as well (Fig. 8, dashed green line). Without refilling correction, a simple '2-exponential' regression clearly misses the time course of release (Fig. 8 , dashed green and black trace, respectively), and a '2-exponential + line' regression was needed (dashed red trace). But for the refilling corrected time course of cumulative release (Fig. 8, grey trace), a '2-exponential' regressions was sufficient (dashed orange trace). The yielded fitting parameters are listed in Table 2-4, and table entries for the finally determined type of release are highlighted red for uncorrected, '2-exponential + linear' release time 


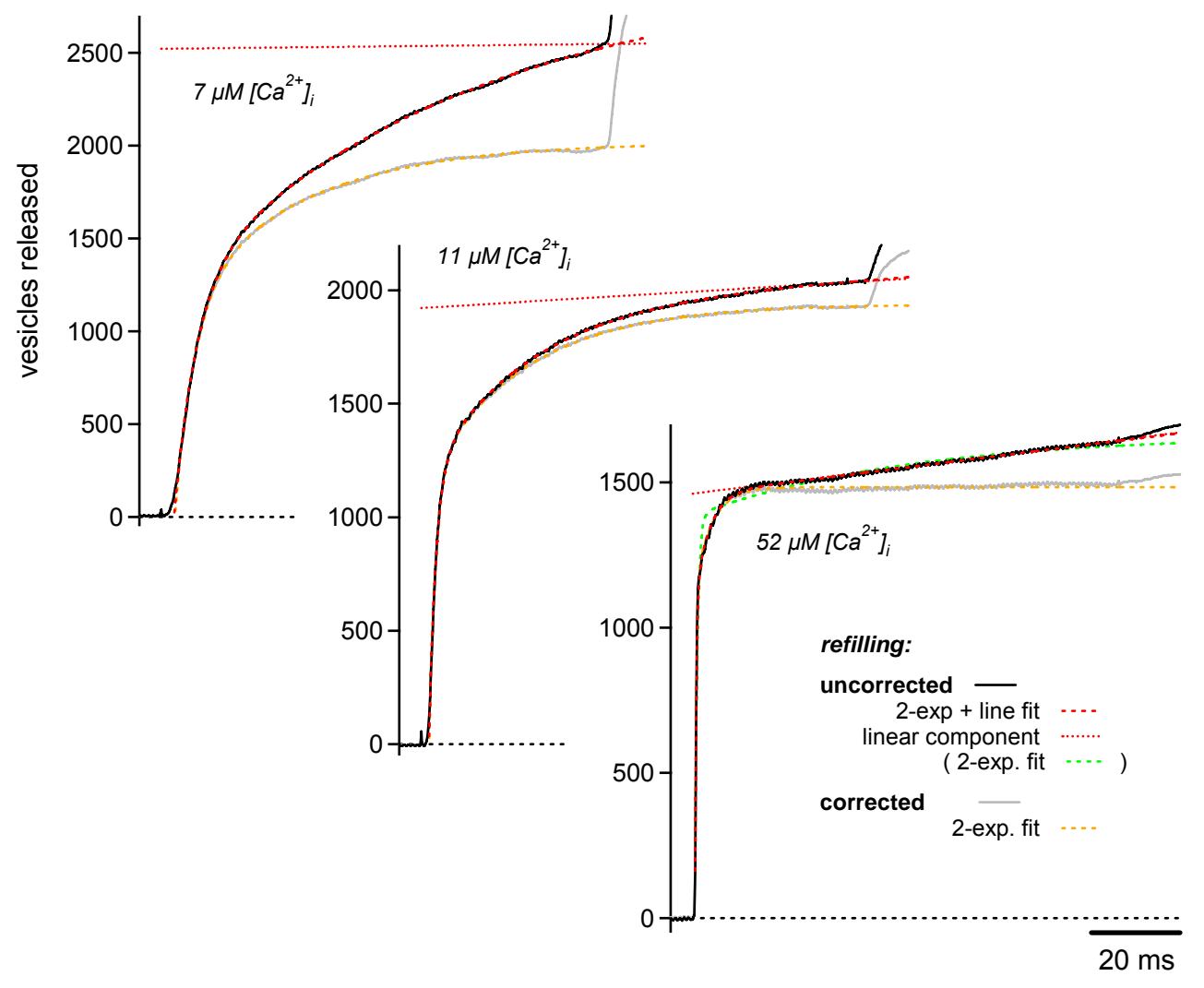

Fig. 8 Approximating refilling with different approaches during analysis Time courses of release at three different stimulus intensities $\left(7 \mu \mathrm{M}, 11 \mu \mathrm{M}, 52 \mu \mathrm{M}\left[\mathrm{Ca}^{2+}\right]_{\mathrm{i}}\right)$ without and with initial refilling correction (black and grey traces, respectively) are shown. Refilling corrected traces were classified as '2-exponential' (dashed orange traces), and uncorrected traces as '2-exponential + line' (dashed red traces). The linear components of the '2-exponential + line' fits are shown as well (dotted red lines), and for the strongest stimulus, the '2-exponential' fit to the uncorrected time course of release is displayed, too (dashed green trace). Responses to the stimuli to $7 \mu \mathrm{M}$ and $11 \mu \mathrm{M}\left[\mathrm{Ca}^{2+}\right]_{\mathrm{i}}$ have been recorded in the same cell.

courses, and orange for refilling corrected, '2-exponential' release time courses (same color code as for fits in Fig. 8).

At the mid and the high stimulus strength of $11 \mu \mathrm{M}$ and $52 \mu \mathrm{M}\left[\mathrm{Ca}^{2+}\right]_{\mathrm{i}}$, the results from both refilling approximation methods yielded very similar results. Estimated time constants and pool sizes of the fast and the slow release component scarcely differed, so in this $\left[\mathrm{Ca}^{2+}\right]_{\mathrm{i}}$ range the validity of fitting a regression with an intrinsic linear component could be confirmed by an alternative analysis approach. 


\begin{tabular}{|c|c|c|c|c|c|c|c|c|c|}
\hline$[\mathrm{Ca} 2+] \mathrm{i}$ & $\begin{array}{c}\text { refilling } \\
\text { corrected }\end{array}$ & fit type & $\begin{array}{r}\tau_{\text {fast }} \\
(\mathrm{ms}) \\
\end{array}$ & $\begin{array}{r}\tau_{\text {slow }} \\
(\mathrm{ms})\end{array}$ & $\begin{array}{c}A_{\text {fast }} \\
\text { (ves.) }\end{array}$ & $\begin{array}{c}A_{\text {slow }} \\
\text { (ves.) }\end{array}$ & $\begin{array}{r}\text { delay } \\
(\mathrm{ms}) \\
\end{array}$ & $\begin{array}{r}\text { line slope } \\
\text { (ves/ms) }\end{array}$ & $\begin{array}{l}\mathrm{A}_{\text {fast }} / \\
\text { total }\end{array}$ \\
\hline \multirow{4}{*}{$7 \mu \mathrm{M}$} & no & 2-exp. & 5.04 & 68.85 & 1245 & 1679 & 2.91 & - & $43 \%$ \\
\hline & no & $\begin{array}{l}\text { 2-exp. + } \\
\text { line }\end{array}$ & 5.04 & 67.23 & 1243 & 1630 & 3.01 & 0.26 & $43 \%$ \\
\hline & yes & 2-exp. & 4.40 & 28.08 & 1157 & 848 & 3.07 & - & $58 \%$ \\
\hline & yes & $\begin{array}{c}\text { 2-exp. + } \\
\text { line }\end{array}$ & 4.45 & 44.67 & 1235 & 1173 & 2.91 & -3.22 & $51 \%$ \\
\hline \multirow{4}{*}{$11 \mu \mathrm{M}$} & no & 2-exp. & 1.62 & 28.24 & 1290 & 827 & 1.61 & - & $61 \%$ \\
\hline & no & $\begin{array}{c}\text { 2-exp. + } \\
\text { line }\end{array}$ & 1.55 & 22.86 & 1261 & 736 & 1.63 & 1.18 & $63 \%$ \\
\hline & yes & 2-exp. & 1.53 & 22.28 & 1197 & 739 & 1.71 & $=$ & $62 \%$ \\
\hline & yes & $\begin{array}{c}\text { 2-exp. }+ \\
\text { line }\end{array}$ & 1.54 & 22.84 & 1200 & 747 & 1.71 & -0.13 & $62 \%$ \\
\hline \multirow{4}{*}{$52 \mu \mathrm{M}$} & no & 2-exp. & 0.54 & 4.86 & 1389 & 277 & 0.43 & - & $83 \%$ \\
\hline & no & $\begin{array}{c}\text { 2-exp. + } \\
\text { line }\end{array}$ & 0.26 & 3.15 & 1104 & 358 & 0.49 & 1.91 & $76 \%$ \\
\hline & yes & 2-exp. & 0.28 & 3.83 & 1133 & 346 & 0.49 & $=$ & $77 \%$ \\
\hline & yes & $\begin{array}{c}2-\exp .+ \\
\text { line }\end{array}$ & 0.26 & 3.15 & 1105 & 358 & 0.49 & 0.31 & $76 \%$ \\
\hline
\end{tabular}

Table 2-4 Parameters from fits to uncorrected and refilling corrected cumulative release time courses at three different $\left[\mathrm{Ca}^{2+}\right]_{\mathrm{i} .}$ Results from fit routines applied to the three traces in Fig. 8 are displayed.

However, for the stimulus to the low $\left[\mathrm{Ca}^{2+}\right]_{\mathrm{i}}$ of $7 \mu \mathrm{M}$, results by the two approaches differed slightly for the fast, and more clearly for the slow component of release. After initial refilling correction, the estimate for the fast component had about the same size, but speeded up by $13 \%$, while the estimate for the slow component was reduced to about half from 1630 vesicles to 850 vesicles, and markedly sped up by $58 \%$ from $67 \mathrm{~ms}$ to $28 \mathrm{~ms}$, as compared to the refilling approach without initial refilling correction. This difference in the two approaches can be explained by the convergence of the release rates of the slow component and the refilling component at low stimulus strengths. Maximal fast refilling of the slow component was estimated to be about $10 \mathrm{ves} / \mathrm{ms}$ (Sakaba and Neher, 2001b), and with an assumed pool size of the slow component of about 1300 vesicles (Fig. 14E), this implied a minimal time constant of refilling of about $70 \mathrm{~ms}$ (for calculation see equation to yield $\tau$, this chapter). Thus, while the fast release component did not reach such low release rates in the range of stimulus intensities applied (see Fig. 14A), the slow component might have kinetically mixed up with its own refilling below about $10 \mu \mathrm{M}\left[\mathrm{Ca}^{2+}\right]$. So, an a priori correction 
for refilling would have lead to better fitting results in the first instance. But since the release kinetics of the slow component and its own refilling were, if at all, hardly distinguishable at low stimulation strengths, an a priori refilling correction might not only have subtracted the refilling, but could at least partially have also quenched release from the initially available slow release component, thus leading to an underestimation of the size of the slow release component, and concomitantly also of the release time constant. On the other hand, comparison of the size of the slow release component estimated at $7 \mu \mathrm{M}\left[\mathrm{Ca}^{2+}\right]_{\mathrm{i}}$ to estimates at $11 \mu \mathrm{M}$ (Table 2-4; both stimulus responses were recorded from the same cell) showed, that the initial refilling correction approach led to more consistent pool size estimates in contrast to the other approach.

Finally, estimates of the slow release component were stable at moderate to high $\left[\mathrm{Ca}^{2+}\right]_{\mathrm{i}}$ above about $10 \mu \mathrm{M}$, while below this stimulus intensity, release kinetics and size of the slow release component could not be extracted adequately by the approaches tested. Thus in

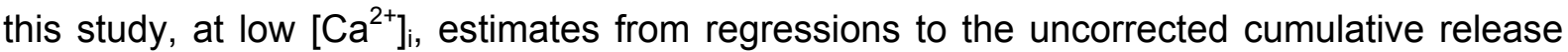
rather underestimated the speed of release from the slow release component, and overestimated the corresponding amounts of slow release.

\subsubsection{Presynaptic membrane capacitance measurements}

Presynaptic membrane capacitance $\left(C_{m}\right)$ measurements were made as described by Wölfel and Schneggenburger (2003; see also chapter 2.2). For an estimate of total release, changes in $\mathrm{C}_{\mathrm{m}}$ were calculated by calculating the difference in $\mathrm{C}_{\mathrm{m}}$ estimates before and after stimulation. For the baseline, $C_{m}$ was averaged in a range from $40 \mathrm{~ms}$ to $20 \mathrm{~ms}$ before stimulation, and for the post-stimulus estimate, $C_{m}$ was averaged between $105 \mathrm{~ms}$ and 125 ms after onset of the stimulus (Fig. 11B). This time range was chosen, because the artifact on $C_{m}$ measurements due to large EPSCs already had ceased (see chapter 3.2 and Fig. 13), and the presynaptic current transients have decreased sufficiently (Wölfel et al., 2003), and endocytotic effects should still be minor (Sun and Wu, 2001; but see also Sun et al., 2002).

\subsubsection{Large postsynaptic EPSCs leading to amplifier non-linearities}

In some cells, peak amplitudes of recorded EPSCs exceeded $-14 \mathrm{nA}$, and beyond this limit amplifier saturation occurred (Fig. 9A, continuous grey trace), being apparent as a sudden decease in the EPSC slope. This happened in the typically used mid gain range (0.5 - 20 
$\mathrm{mV} / \mathrm{pA}$; feedback resistor $R_{f}=500 \mathrm{M} \Omega$ ) when $R_{\mathrm{s}}$-compensation was turned on (chapter 2.2), and was due to the hardware design of the HEKA EPC-9/2 patch-clamp amplifier.

To examine the impact of peak EPSC saturation, the corresponding not saturated response has been approximated by linearly scaling the $R_{\mathrm{s}}$-compensation corrected EPSC (Fig. 9A, black trace) in the region of saturation (dotted lines). The scaling factor was changed until the course of the rising EPSC became as smooth as possible, and no edge was visible anymore as estimated by eye (Fig. 9A, red trace; see also dashed lines in magnified view below). In this example, the factor was set to 2.5. Then, both traces, corrected and uncorrected, were deconvolved and analyzed as usual. The time course of release was determined to be '2exponential + line' in both cases, but the estimated release parameters differed. It turned out that by amplifier saturation, release estimated for the fast component became slower by about $35 \%$, and decreased in its size by $8 \%$. The slow component speeded up by about $25 \%$, and its size increased by $11 \%$, while the amount of total release stayed constant. This was expected, because a decreased peak EPSC amplitude should have led to a shallower raise in the cumulative release for the time range affected by saturation, such that the fast component from fitted regressions slowed down, while the slow component was accelerated during saturation. As a result, release from the artificially slowed fast component will be partially mistaken to belong to the slow component during analysis. The amount of total release should be slightly reduced by amplifier saturation, because the EPSC charge will be reduced, and also the estimated residual current will differ accordingly.

Because of this dramatic effect of amplifier saturation onto estimates of release amplitudes and time courses, data showing this detriment have not been included in any further analysis. However, the use of $\gamma$-DGG (chapter 2.1) reduced EPSC sizes, and therefore avoided amplifier saturation in most recordings. Data with amplifier saturation will be shown only in a few single cell example plots, when they nicely and convincingly demonstrate the matter of interest (Figs. 10, 16, 21). But still, their results were not included in further analysis or any other plots. 

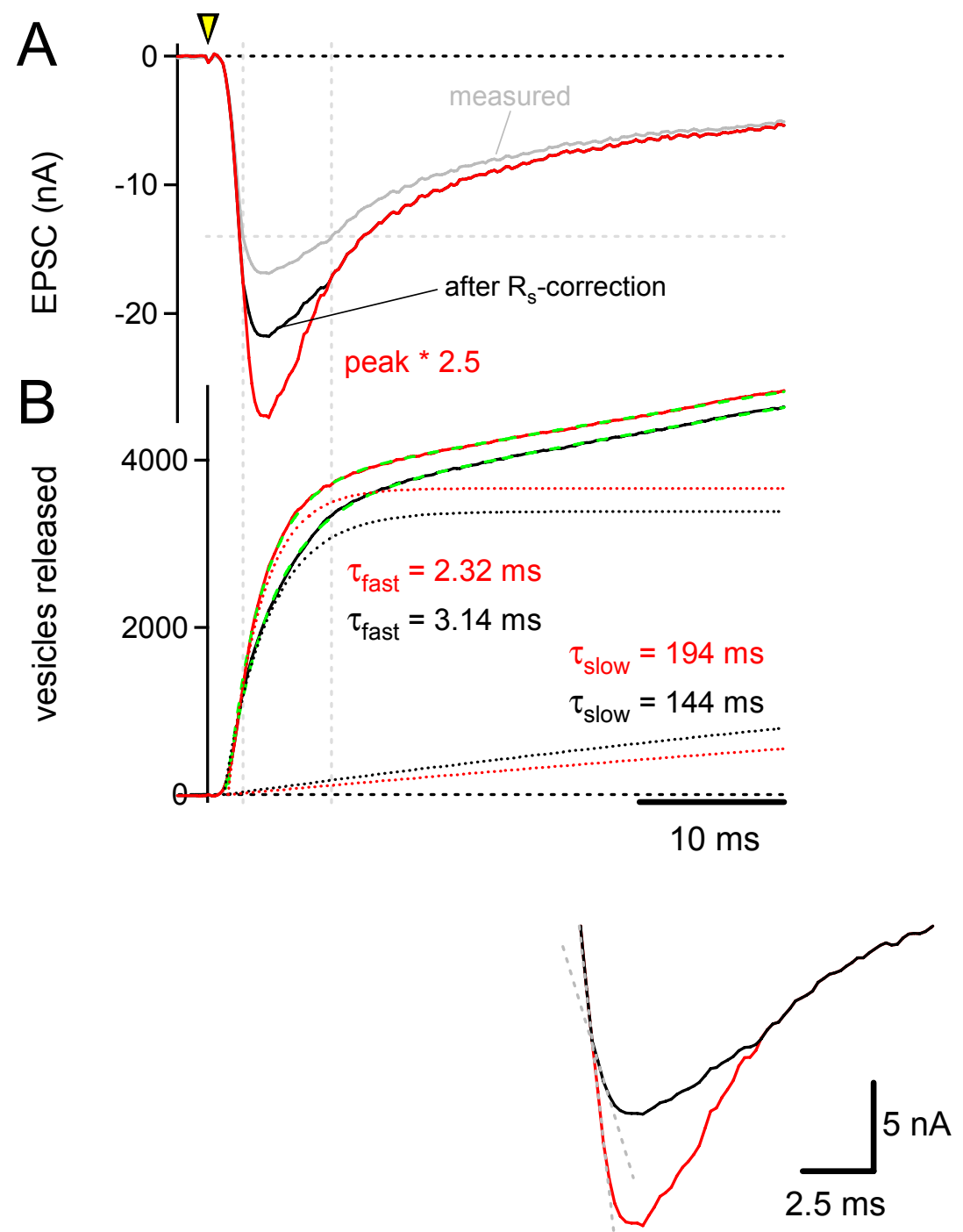

Fig. 9 Amplifier saturation for EPSCs larger than -14 nA

A An EPSC before $R_{\mathrm{s}}$-compensation correction (continuous grey trace) shows a dampened amplitude when exceeding $-14 \mathrm{nA}$ (horizontal dashed grey line). The $\mathrm{R}_{\mathrm{s}}$-compensation corrected EPSC (black trace) has been scaled by a factor of 2.5 (red trace) for the range of saturation (vertical dashed grey lines). The magnified view below shows the EPSC peak amplitudes on an expanded scale, for clarity. B The corresponding time courses of release (black traces) were fitted (green traces), and fast and slow components are displayed separately (dotted traces). 


\section{Experimental results}

\subsection{Depolarization induced release components}

\subsubsection{Two components of release during AP-like stimuli}

A single AP leads to release of about 150 - 200 vesicles (Borst and Sakmann, 1995; Meyer et al., 2001), and during high activity up to an AP frequency of $600 \mathrm{~Hz}$ (Spirou et al., 1990), postsynaptic currents sum to a synchronous, and an asynchronous component of release (Wu and Borst, 1999). This can also be seen during paired pre- and postsynaptic whole-cell voltage clamp recordings, when a train of APs is mimicked by short depolarizations (Borst and Sakmann, 1995; Borst and Sakmann, 1998).

A train of 15 short depolarizations from a holding potential of $-80 \mathrm{mV}$ to $0 \mathrm{mV}$ for $1 \mathrm{~ms}$ at 100 $\mathrm{Hz}$ was applied at the calyx of Held terminal, and simultaneously, EPSCs were recorded (Fig. 10A, black traces). To prevent postsynaptic AMPA receptor desensitization and saturation, CTZ and $\gamma$-DGG were present in this recording, respectively, and for presynaptic $\mathrm{Ca}^{2+}$ buffering conditions similar to physiological conditions, $200 \mu \mathrm{M}$ EGTA was included in the presynaptic pipette (chapter 2.2). Facilitation and depression could be observed in the first increasing, then decreasing sizes of peak EPSCs (Fig. 10A), and after deconvolution (chapter 2.4.1), both clearly showed up in the release rates of the first few stimuli (Fig. 10B, arrows). The decay phase of peak release rates seemed to be biphasic, indicating two

Fig. 10 AP-like stimuli induce two kinetically distinct components of release

A A train of 15 presynaptic depolarizations to $0 \mathrm{mV}$ for $1 \mathrm{~ms}$ at $100 \mathrm{~Hz}$ was followed by a $50 \mathrm{~ms}$ depolarization (ISI = $20 \mathrm{~ms}$; black traces). A single depolarization to $0 \mathrm{mV}$ for $64 \mathrm{~ms}$ (blue traces) was given in the same cell, as well (stimulus protocols shown on top). Deconvolution of elicited EPSCs (A), yielded release rates (B) and cumulative release (C) as functions of time. During the train, facilitation and depression are visible in the EPSCs and release rate traces (arrows), and the time course of cumulative release shows biphasic kinetics (C). Contributions of fast and slow components are indicated (C, D, dotted and dashed grey lines for FRP and SRP, respectively). During long depolarizations, regressions to the time courses of cumulative release (dashed red curves) could separate between release kinetics and amplitudes from the FRP (dotted red curves) and the SRP. E Release time constants $\tau$ (left panel) and amount of vesicles released (right panel) for the FRP (closed black triangles) and SRP (open black triangles). Averages were $931 \pm 388 \mathrm{FRP}$ vesicles with $3.2 \pm 1.3 \mathrm{~ms}$, and $1751 \pm 1114 \mathrm{SRP}$ vesicles with $38.9 \pm 32.6 \mathrm{~ms}\left(\mathrm{~N}=9 ; \mathrm{p}_{\tau}=0.011\right)$. 
A
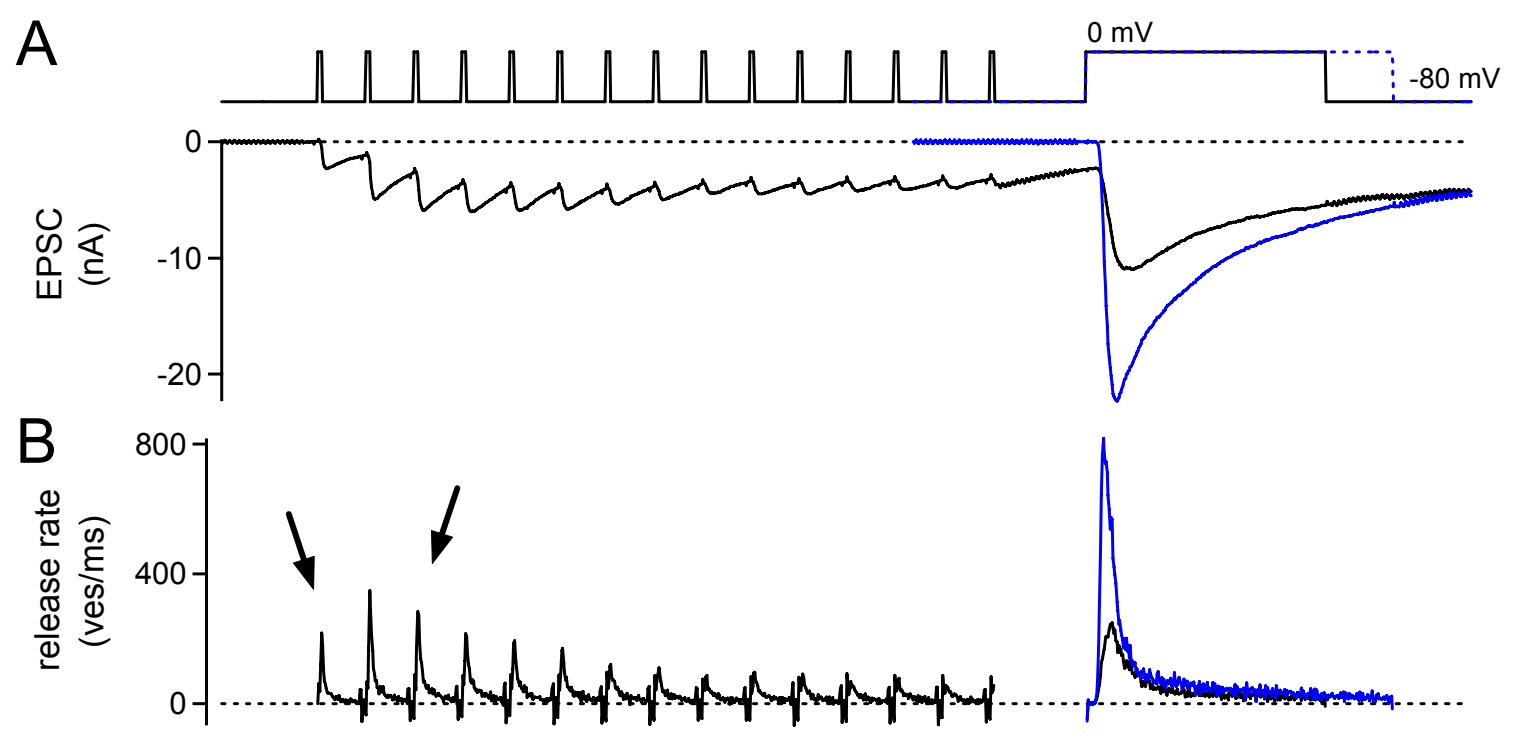

c

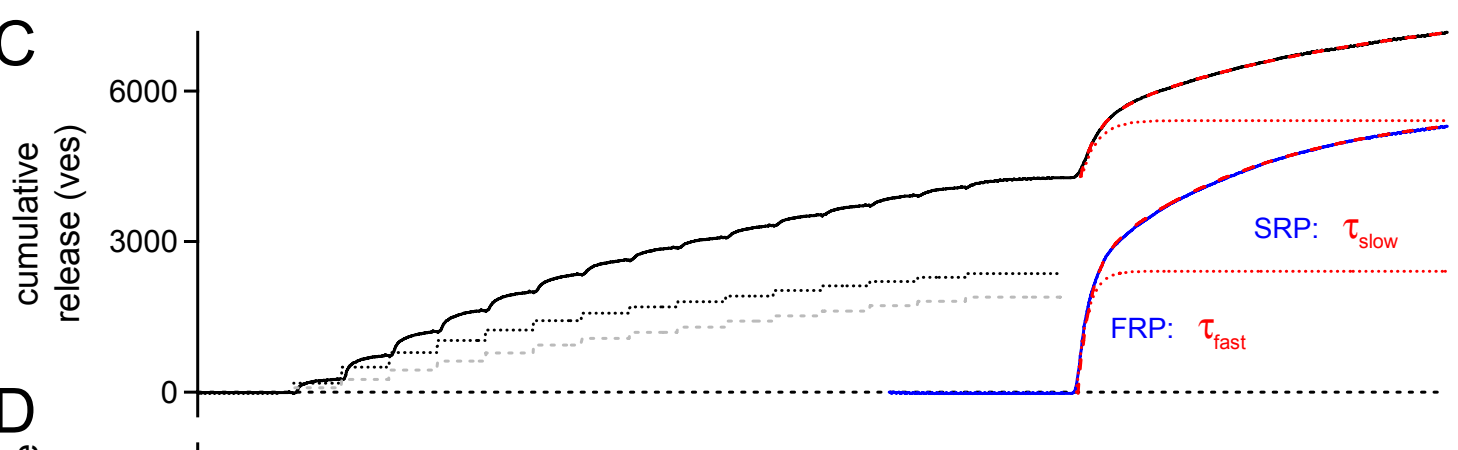

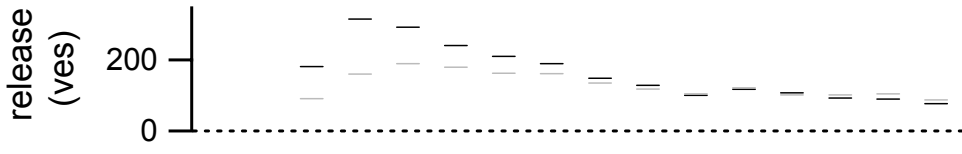

FRP: -
SRP:

$50 \mathrm{~ms}$

E
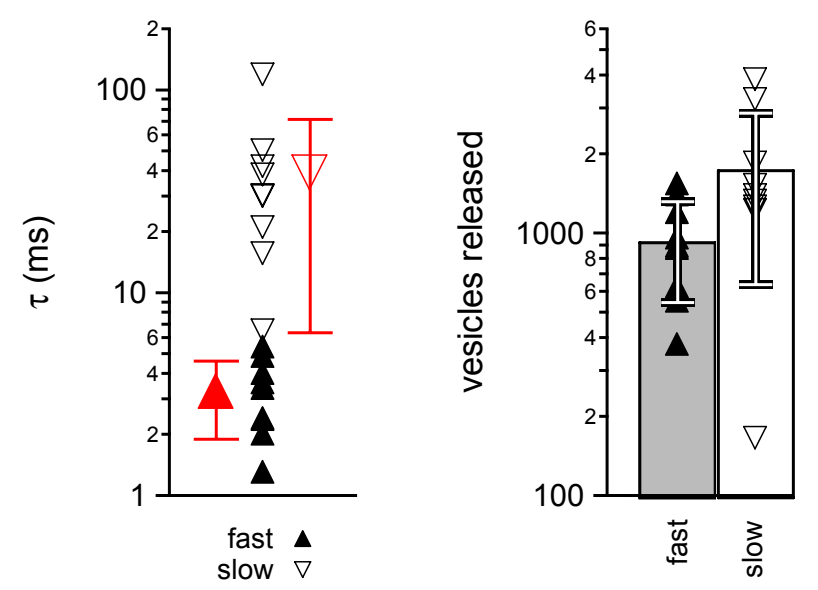
kinetically distinct release components. Two phases of release have also been described before. During high AP frequencies up to $600 \mathrm{~Hz}$ (Spirou et al., 1990), postsynaptic currents sum to a synchronous and an asynchronous component or release (Wu and Borst, 1999).

For further description of biphasic release, as observed here, the two release components were segregated. This was done by defining the initial fast rise in cumulative release as the amount of fast release (Fig. 10C), up to that time point, where the fast decay in the release rate (Fig. 10B) had ended, being 2 ms after onset of each stimulus. The remaining amount of release until onset of the next stimulus was determined to be slow release. Fast and slow release for each single stimulus is shown in Fig. 10D (black and grey symbols, respectively), and also cumulatively in Fig. 10C (dotted black and dashed grey trace, respectively). Again, facilitation and depression became obvious (Fig. 10D), and fast and slow release contributed about equally to total release after the 15 train pulses applied (55\% were fast). The first short pulse induced release of 181 fast releasing vesicles, being in the range of estimates for release during a single AP (150 - 200 vesicles; Borst and Sakmann, 1995; Meyer et al., 2001).

More detailed examination of biphasic release was aimed with a single prolonged depolarization. A depolarization from $-80 \mathrm{mV}$ to $0 \mathrm{mV}$ for $64 \mathrm{~ms}$ was applied at the calyx of Held terminal, and EPSCs were recorded simultaneously (Fig. 10A, blue traces). Such a long stimulus is expected to deplete the readily-releasable pool of vesicles (RRP) completely (Sakaba and Neher, 2001a; Sakaba and Neher, 2001b; Wölfel and Schneggenburger, 2003). As for the train stimulation, during the long depolarization the decay phase of the release rate time course, and the rise of cumulative release showed biphasic kinetics (Fig. 10B, C), comparing well to previous findings (Sakaba and Neher, 2001b). After fitting the time course of cumulative release with exponential regressions (chapter 2.4.2), the rise could be classified as '2-exponential + line' (Fig. 10C, dashed red line on blue trace), with a contribution of $42 \%$ fast vesicles (dotted red line). In this study, henceforth the fast and the slow release component will be termed fast-releasable pool of vesicles (FRP), and slowreleasable pool of vesicles (SRP), respectively. On average, during prolonged depolarizations ( $\geq 50 \mathrm{~ms}$ ), depletion of the FRP occurred with a time constant of $3.2 \pm 1.3 \mathrm{~ms}$, and with $38.9 \pm 32.6 \mathrm{~ms}$ for the SRP (Fig. 10E; $\mathrm{N}=9, \mathrm{p}=0.011$ ). The observed biphasic release time course cannot be described by the simplest depletion model at the calyx of Held synapse (Schneggenburger and Neher, 2000; Bollmann et al., 2000), although being able to describe depression. This model assumes a homogeneous release probability throughout the RRP, but this would also lead to homogeneous release kinetics, being contrasted by the observations made here. 
So, long depolarizations (Fig. 10, blue traces) led to release of two kinetically distinct components with approximately equal contributions to total release. During train stimulation, vesicles were also released fast and slowly. But despite an apparent FRP depletion, a surprisingly large contribution of fast vesicles was left for the subsequent, long depolarization. To explore, why release from the RRP could be partitioned into the FRP and SRP, further experiments were performed.

\subsubsection{Comparing deconvolution results to $C_{m}$ estimates}

To consolidate the finding from EPSC deconvolution that two kinetically distinct release components, the FRP and the SRP, exist, presynaptic membrane capacitance $\left(C_{m}\right)$ measurements were combined with EPSC recordings, and changes in $\mathrm{C}_{\mathrm{m}}$ were compared to the amount of cumulative release calculated by deconvolution. In paired pre- and postsynaptic whole-cell voltage-clamp recordings under CTZ, the presynaptic terminal was depolarized to $0 \mathrm{mV}$ for different stimulation lengths, leading to different amounts of release. In the example shown (Fig. 11A, black traces), depolarizations of 2, 4, 8, 16, and $64 \mathrm{~ms}$ induced biphasic release (continuous black lines on right axis), with amplitudes of release from the FRP indicated (dashed black lines). However, the relative contribution of slow release was smaller for short pulses. For depolarizations longer than the time needed to reach the peak EPSC, the dominance of the fast release component in defining the peak EPSC amplitude becomes obvious. Already with the $4 \mathrm{~ms}$ stimulus, a maximal peak EPSC was reached, and FRP depletion was complete, as compared to longer depolarizations. In contrast, slow release rather took place during the decay of the EPSC, and cessation of the stimulus leads to incomplete depletion of the SRP, thus an accelerated EPSC decay time course. This is illustrated by overlaying the EPSCs in response to short depolarizations (4, $16 \mathrm{~ms}$; Fig. 11A, grey asterisks) over the long pulse of $64 \mathrm{~ms}$ (Fig. 11A, grey traces underneath black line, respectively; see also arrowhead). Also, the missing EPSC response to presynaptic $\mathrm{Ca}^{2+}$ tail currents for stimulations longer than $4 \mathrm{~ms}$ demonstrates the depletion of the FRP, and also indicates the slow kinetics of the SRP that did not change markedly even at this transiently increased stimulus strength.

Since postsynaptic AMPA receptor saturation (Sun and Wu, 2001) could lead to a change in the EPSC time course, possibly being misinterpreted as a second, kinetically distinct release component, this effect was tested by comparing results obtained with and without application of the antagonist $\gamma$-DGG competing with glutamate for AMPA receptors (Fig. 11, red and black data, respectively). Data obtained under $2 \mathrm{mM} \gamma$-DGG yielded comparable results (Fig. 
11C, red and black data, respectively). However, while the blocking efficiency of $\gamma$-DGG (Fig. 11D) for depolarizations of at least $4 \mathrm{~ms}$ was almost constant (52 $\pm 13 \%, N=15$; linear estimate with a slope of $-1.9 \%$ block per ms depolarization), the blocking efficiency during a 2 ms stimulus was somewhat smaller $(29 \pm 20 \%, N=3)$. This is expected, if multivesicular release takes place at relatively strong stimulus intensities (Meyer et al., 2001; Sun and Wu, 2001; Wadiche and Jahr, 2001; Wölfel and Schneggenburger, 2003). $\gamma$-DGG competes with glutamate in a concentration dependent manner, and at low stimulus intensities, low release probabilities will induce fusion of less than 3 - 4 vesicles at an active zone (Meyer et al., 2001; Sun and Wu, 2001; Wölfel and Schneggenburger, 2003), thus the lower glutamate concentration will lead to higher blocking efficiencies of competitive antagonists.

The slow release phase detected by EPSC deconvolution took place during the decaying phase of the EPSC. Essentially, the slower decay of the EPSC evoked by the 64 ms stimulus, as compared to EPSCs induced by shorter pulses (Fig. 11A, compare rightmost black and grey EPSC traces) was interpreted by deconvolution as additional, late release. However, at this time, AMPA-receptors might have undergone desensitization, which might have led to a serious underestimation of the late release. Therefore, we were interested in estimating late release with an independent method, using presynaptic $\mathrm{C}_{m}$ measurements (Sun and $\mathrm{Wu}$, 2001; Wölfel and Schneggenburger, 2003).

Fig. 11 Cumulative release estimated by EPSC deconvolution and $C_{m}$ measurements Presynaptic depolarizations were analyzed for kinetically different release components using EPSC deconvolution (A), and $C_{m}$ measurements (B). Postsynaptic glutamate receptor saturation was tested by comparing results obtained without $\gamma$-DGG (A - C, E, black data), or with $2 \mathrm{mM} \gamma$-DGG (A - C, E, red data). A Depolarizations to $0 \mathrm{mV}$ for durations of 2, 4, 8, 16, or $64 \mathrm{~ms}$ induced presynaptic $\mathrm{Ca}^{2+}$ currents (top), and EPSCs (middle), which then have been deconvolved to get release rates (bottom, dotted traces) and cumulative release as functions of time (bottom, continuous traces). EPSCs due to $4 \mathrm{~ms}$, and $16 \mathrm{~ms}$ stimulation (grey asterisks) are displayed again underneath the EPSC during $64 \mathrm{~ms}$ stimulation (grey traces at black arrowhead). $B C_{m}$ changes are plotted (different time scale than in A), and estimates for release were made in a time window of $20 \mathrm{~ms}$ starting $105 \mathrm{~ms}$ after onset of stimulation. C Amounts of release in dependence of duration of stimulation are compared for deconvolution results (closed symbols), and $\mathrm{C}_{\mathrm{m}}$ estimates (open symbols). Bi-exponential fits to the data are shown (continuous and dashed blue curves for deconvolution and $C_{m}$ results, respectively). D Blocking efficiency of $\gamma$-DGG in dependence of stimulus duration. Peak EPSCs under $2 \mathrm{mM} \gamma$ DGG were normalized to peak EPSCs without $\gamma$-DGG in the same cell ( $N=3$ cells). $E$ Correlation of release estimates from deconvolution and $C_{m}$ measurements at given stimulus lengths. 

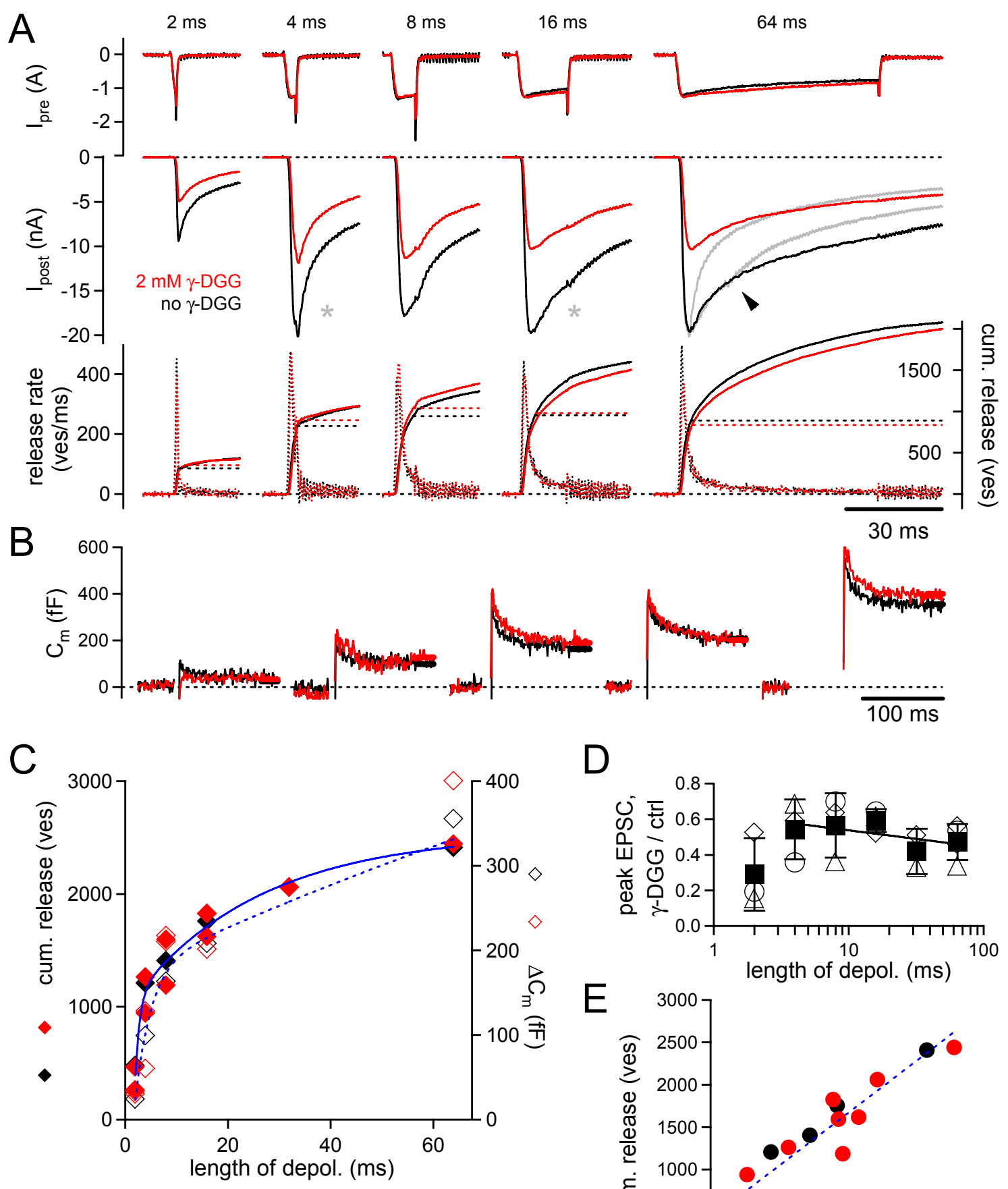

D
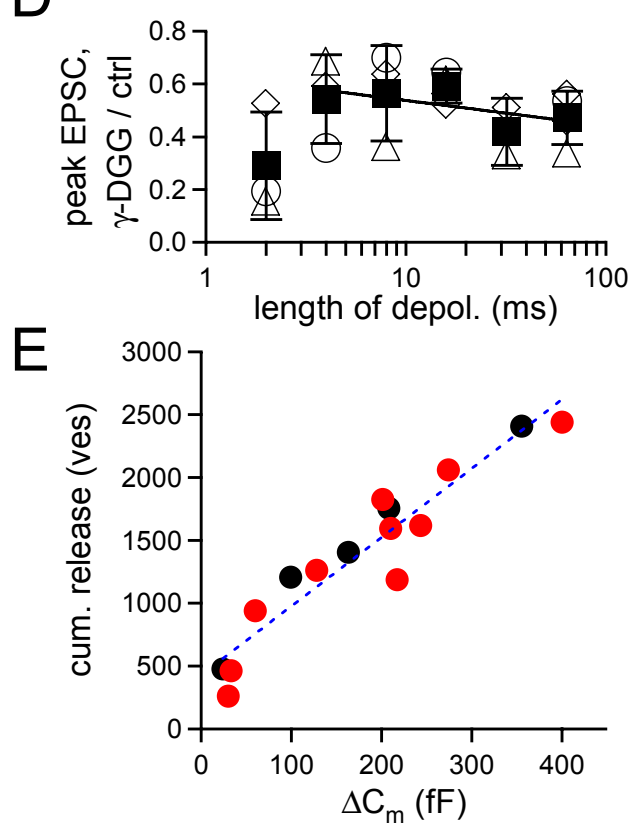
Since $C_{m}$ estimates can be achieved only after, but not during depolarizations (Gillis, 1995; Sun and Wu, 2001; Wölfel and Schneggenburger, 2003) the amount of release was read out from $\mathrm{C}_{\mathrm{m}}$ recordings $100 \mathrm{~ms}$ after onset of stimulation. Corresponding estimates were received form cumulative release traces derived by EPSC deconvolution (Fig. 11A and 11B, respectively; note different time scales). Plotting these estimates against the different durations of stimulation (Fig. 11C) led to a reasonable correlation between estimated amounts of release from deconvolution, and from $\mathrm{C}_{\mathrm{m}}$ analysis (closed and open symbols, respectively). Both methods unveiled the biphasic behavior of release, with a fast component depleted by stimulations of about $8 \mathrm{~ms}$, and a slow component with about 10-fold slower kinetics, becoming prominent for depolarizations longer than $16 \mathrm{~ms}$.

A two-exponential regression to the correlation between cumulative release and stimulation length (Fig. 11C, continuous blue line) resulted in estimates for the FRP of 980 vesicles with a release time constant of $0.88 \mathrm{~ms}$, and for the SRP of 1672 vesicles with $22.9 \mathrm{~ms}$. The same regression, but using estimates from $C_{m}$ measurements assessed an FRP of $140 \mathrm{fF}$ with a release time constant of $2.6 \mathrm{~ms}$, and an SRP of $479 \mathrm{fF}$ with $105 \mathrm{~ms}$. The estimate of the SRP release time constant was larger in $C_{m}$ measurements compared to EPSC deconvolution results. This might be due to the excessive changes in the $C_{m}$ estimate during very long depolarizations (Fig. 11C, $64 \mathrm{~ms}$ depolarization), which were possibly caused by $\mathrm{Ca}^{2+}$ induced conductance changes in the presynaptic membrane (see also Wölfel and Schneggenburger, 2003). As a consequence, this artifact taking place only at high stimulus intensities would change the shape of the correlation between changes in $C_{m}$ and stimulus length to slower kinetics. On average, EPSC deconvolution yielded an FRP size estimate of $1972 \pm 1286$ vesicles with a release time constant of $1.5 \pm 1.2 \mathrm{~ms}$, and an SRP size of 3523 \pm 2510 vesicles with $42.5 \pm 46.9 \mathrm{~ms}(\mathrm{~N}=4) . \mathrm{C}_{\mathrm{m}}$ estimates yielded $114 \pm 56 \mathrm{fF}$ with a release time constant of $2.3 \pm 1.4 \mathrm{~ms}$ for the FRP, and $379 \pm 180 \mathrm{fF}$ with $48.6 \pm 49.5 \mathrm{~ms}$ for the SRP $(\mathrm{N}=3)$.

When plotting cumulative release as a function of $C_{m}$ changes (Fig. 11E), the amounts of release estimated from both methods had a linear correlation with a slope of 6.0 vesicles per fF for the example cell shown, and on average $9.9 \pm 6.9$ ves/fF $(N=3)$. This gave a quantal size estimate of about $100 \mathrm{aF}$, being higher but in the same order of magnitude as previous estimates of about $65 \mathrm{aF}$ (Sun et al., 2002). With an estimate of 65 aF/ves, a pool size of $1754 \pm 862$ FRP vesicles could be derived, very similar as the estimate from EPSC deconvolution. But the size of $5831 \pm 2769$ slow vesicles is markedly bigger, most likely because of the high conductance changes during very long depolarizations as discussed before. 
$\mathrm{C}_{\mathrm{m}}$ measurements clearly showed that a second, slow release component exists, exerting its influence mainly after the peak of the EPSC, as predicted by deconvolution. Thus, EPSC peak amplitudes cannot be used as a faithful measure of release (Neher and Sakaba, 2001; but see also Sun and $\mathrm{Wu}, 2001)$. Moreover, the consistence of estimates from $\mathrm{C}_{\mathrm{m}}$ measurement and EPSC deconvolution made an effect of AMPA-receptor desensitization (Sun and $\mathrm{Wu}, 2001$ ) unlikely. Yet, the origin of this slow release component remained unidentified, and a distinction between refilling of the fast release component, or a different process could not be made. But an artificial appearance of the slow release component due to AMPA-receptor saturation, or desensitization was not likely, since data obtained with and without 2 mM $\gamma$ DGG (Fig. 11, red and black data, respectively) yielded very similar results, and $\mathrm{C}_{\mathrm{m}}$ measurement confirmed estimates by EPSC deconvolution. Therefore, in the remaining part of this study, EPSC deconvolution was used for measuring fast and slow release components.

\subsection{Two release components during $\mathrm{Ca}^{2+}$ uncaging}

The appearance of kinetically heterogeneous release during presynaptic depolarizations (chapter 3.1; Sakaba and Neher, 2001b) raises the question of the underlying mechanism. Two major hypotheses are (see also introduction): 1) differential localization of vesicles relative to voltage activated $\mathrm{Ca}^{2+}$ channels (positional heterogeneity; Neher and Zucker, 1993; Neher, 1998), or 2) a differential state of the release machinery shifting its $\mathrm{Ca}^{2+}$ sensitivity (intrinsic heterogeneity). These two mechanisms can account for two release components during depolarizations. But for a spatially uniform $\mathrm{Ca}^{2+}$ signal, positional heterogeneity should convert into homogeneous release, whereas intrinsic heterogeneity still would persist.

Therefore, $\mathrm{Ca}^{2+}$ uncaging experiments were performed in calyces, and postsynaptic recordings were analyzed for presynaptic release components. In clearly identified calyces of Held (e.g. in Fig. 12A, left image, fura-2FF excitation), UV-flash stimuli of different intensities led to different increases in the presynaptic $\left[\mathrm{Ca}^{2+}\right]_{i}$. In the example shown (Fig. 12), a weak stimulus to $5 \mu \mathrm{M}\left[\mathrm{Ca}^{2+}\right]_{\mathrm{i}}$ (Fig. 12B) induced a slowly increasing EPSC. Deconvolution yielded a peak release rate of 34 ves/ms, which decayed slowly. The cumulative release was fitted with different types of regressions ('linear', '1-exp.', '1-exp. + line', '2-exp.', '2-exp. + line', and '3-exp.'), in a time window of $100 \mathrm{~ms}$ after onset of stimulation, and the best fit was 
determined (Methods, chapter 2.4.2). For both stimuli shown (Fig. 12B and C, magnified in D) here, the '2-exponential + line' fit gave the best approximation (dashed red trace), revealing two components of release. In response to the weaker stimulus to $5 \mu \mathrm{M}\left[\mathrm{Ca}^{2+}\right]_{\mathrm{i}}$ (Fig. 12B), a fast release component with 253 vesicles and a release time constant of $6.6 \mathrm{~ms}$, and a slow one with 289 vesicles with $95 \mathrm{~ms}$ appeared (dotted red traces). A stronger stimulus to $22 \mu \mathrm{M}\left[\mathrm{Ca}^{2+}\right]_{\mathrm{i}}$ in the same cell (Fig. 12C) led to faster release with a peak release rate of 509 ves $/ \mathrm{ms}$. The resulting fast component accelerated to $0.9 \mathrm{~ms}$ with 599 vesicles, and the slow component to $41.6 \mathrm{~ms}$ with 479 vesicles.

To verify the release time constant estimates obtained from EPSC deconvolution, comparisons to the time course of $\mathrm{C}_{\mathrm{m}}$ changes were made. As described by Wölfel and Schneggenburger (2003), exponential regressions to the rise in $C_{m}$ in single presynaptic voltage-clamp recordings can yield release time constants after $\mathrm{Ca}^{2+}$ uncaging stimuli. However, here paired pre- and postsynaptic voltage-clamp recordings were made, and artifacts appeared during the rising phase of $C_{m}$ (Fig. 13). The maximum of these artifacts correlated with the time point of the steepest rise in the corresponding EPSC, around $0.2 \mathrm{~ms}$ to $5 \mathrm{~ms}$ after onset of the EPSC, depending on the presynaptic stimulus strength. Thus, in paired pre- and postsynaptic voltage-clamp recordings, simultaneous estimates of release time constants by EPSC deconvolution and $\mathrm{C}_{\mathrm{m}}$ measurements could not be achieved, in contrast to experiments where postsynaptic glutamate receptors were blocked (Wölfel and Schneggenburger, 2003: $10 \mu \mathrm{M} N B Q X$ and $50 \mu \mathrm{M}$ D-AP5 to block AMPA- and NMDAreceptors, respectively). Since EPSC deconvolution yielded release time courses with a

Fig. $12 \mathrm{Ca}^{2+}$ uncaging elicits two kinetically distinct components of presynaptic release $\mathrm{Ca}^{2+}$ uncaging and $\left[\mathrm{Ca}^{2+}\right]_{i}$ experiments were performed in the calyx of Held (A Fura-2FF fluorescence images at $380 \mathrm{~nm}$; left: $640 * 480$ pixel image, right: $8 * 15$ binned image as during $\left[\mathrm{Ca}^{2+}\right]_{i}$ measurements; scale bar $\left.10 \mu \mathrm{m}\right)$. B, C Two Ca ${ }^{2+}$ uncaging stimuli (arrowheads) of different intensities $\left(5 \mu \mathrm{M}\right.$ and $\left.22 \mu \mathrm{M}\left[\mathrm{Ca}^{2+}\right]_{\mathrm{i}}\right)$ were applied within the same cell, and time courses of cumulative release were classified as '2-exp. + line' in either case (dashed red curves). Release from the FRP and SRP are indicated (dotted red curves). Residual currents estimated by EPSC deconvolution are displayed as well (dashed black traces). Line fits for slow release rate estimates are shown (dashed green lines). For comparison of both responses, EPSC and release rates shown in B are plotted again in C (grey traces). D time course of cumulative release for both stimuli are displayed together with all regressions made. Please note that at low $\left[\mathrm{Ca}^{2+}\right]_{\mathrm{i}}$ (left panel), the '1-exponential + line' fit (dashed, light blue curve) could not follow the late release time course later than $40 \mathrm{~ms}$ after stimulation. 
A
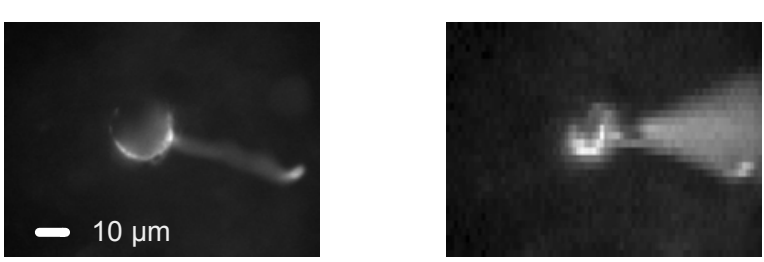

B
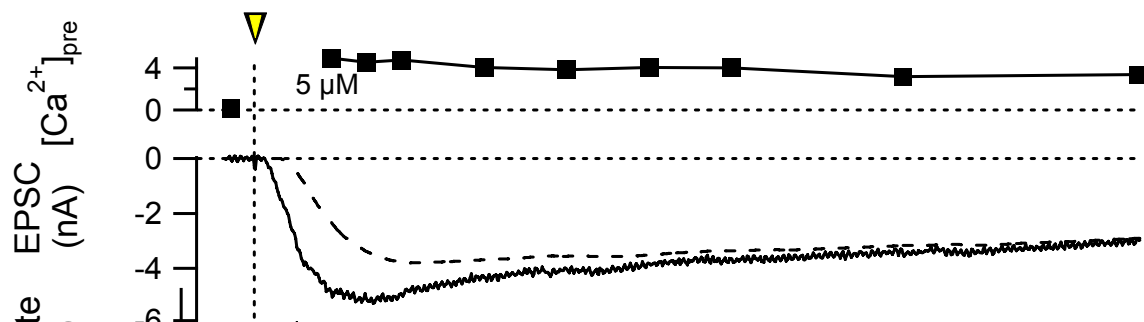

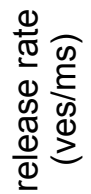

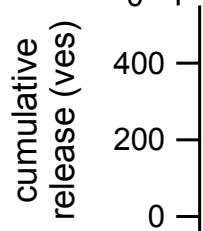
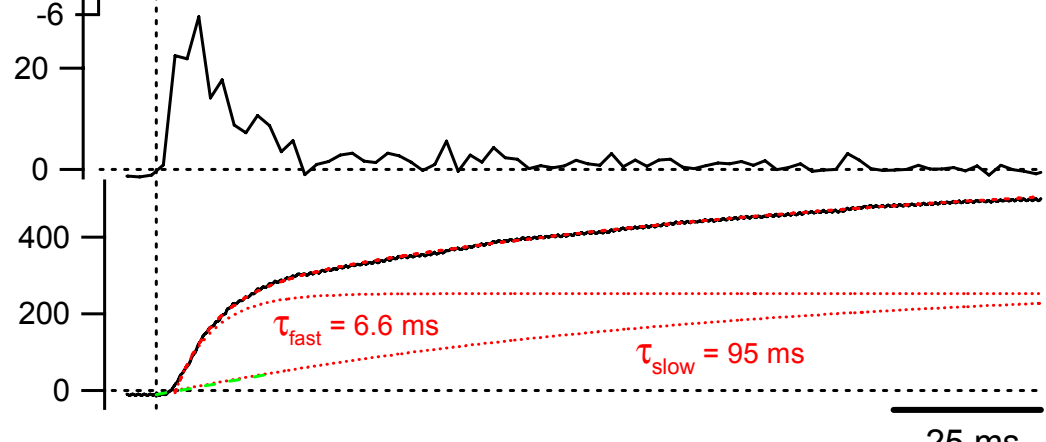

C
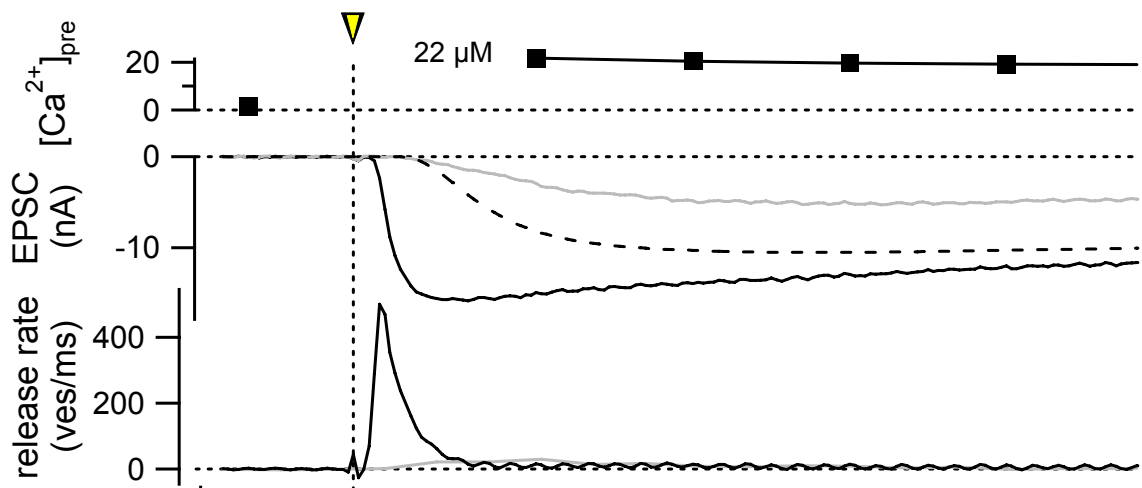

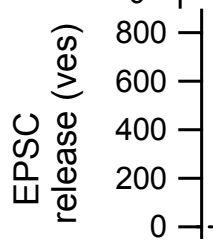

D
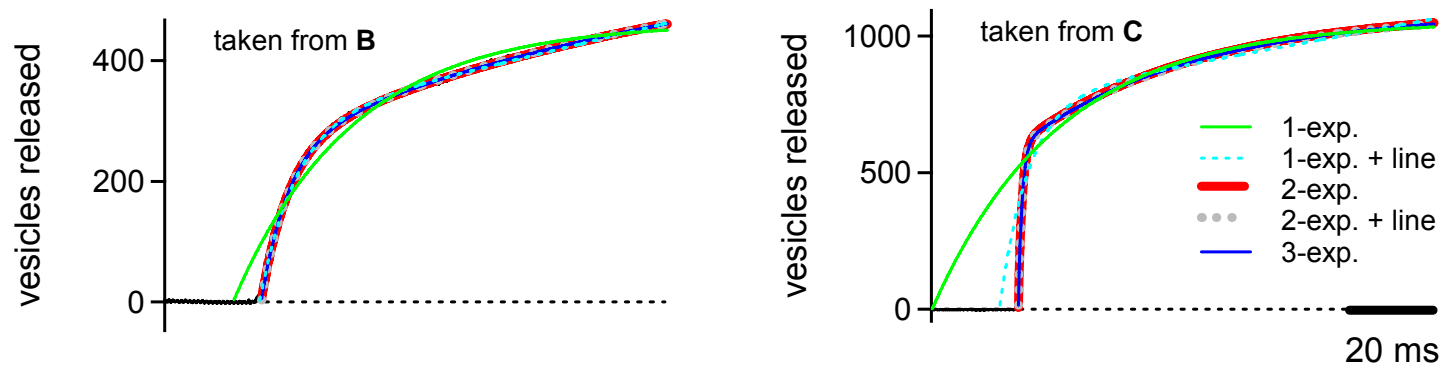
better sampling resolution and less noise, as compared to $C_{m}$ measurements, they were preferred for release kinetic estimates in this study. However, because the described artifact correlated with the change in the EPSC, and therefore was transient, absolute estimates of total vesicles released in dependence of the stimulation strength were still possible (as shown in Fig. 11).
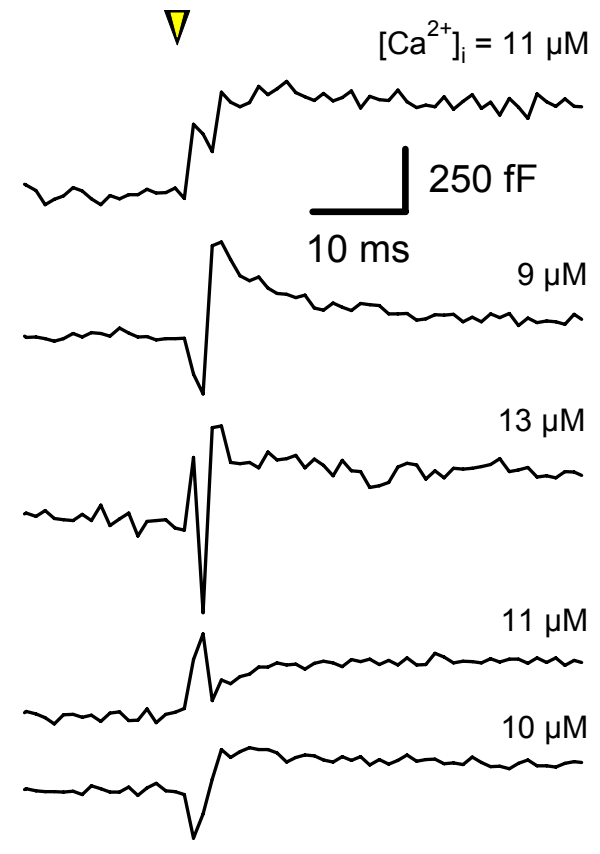

Fig. 13 Transient artifacts on $\mathrm{C}_{\mathrm{m}}$ estimates due to fast changes in the EPSC Five presynaptic $C_{m}$ recordings from different cells, and the corresponding $\left[\mathrm{Ca}^{2+}\right]_{i}$ after $\mathrm{Ca}^{2+}$ uncaging (arrowhead) are shown. Traces were obtained under paired pre- and postsynaptic whole-cell voltage-clamp recordings. An artifact on $\mathrm{C}_{\mathrm{m}}$ estimates frequently occurred, when the slope of the EPSC rise was steepest, probably due to electric field forces of large EPSCs of several nA.

Fig. 14 Two kinetically distinct components of release during $\mathrm{Ca}^{2+}$ uncaging Results from analyzed time courses of cumulative release were summarized ( $N=104)$, and extracted time constants of release (A), peak release rates (B), delays (C), times-to-peak release rates (D), and amounts of vesicles released (E) are displayed. The best type of regression to each response is specified by the respective symbol (see textbox). For the total dataset, the contribution of each fit type is indicated, as well. In the range of $10 \mu \mathrm{M}$ to $15 \mu \mathrm{M}$ $\left[\mathrm{Ca}^{2+}\right]_{\mathrm{i}}$, averaged data for the FRP and SRP (from all responses with two release components) yielded time constants of $\tau=1.9 \pm 1.2$ and $43.3 \pm 50.0 \mathrm{~ms}(\mathrm{~A})$, peak release rate $=806 \pm 577$ and $41 \pm 55 \mathrm{ves} / \mathrm{ms}(\mathrm{B})$, and vesicles released $=1491 \pm 778$ and $1255 \pm 992(\mathrm{E})$, respectively $(\mathrm{N}=18)$. Additionally for the FRP, the averaged delay was $1.4 \pm 0.4 \mathrm{~ms}(\mathrm{C})$, and the time-topeak release rate equaled $2.0 \pm 0.8 \mathrm{~ms}$ (D). In $\mathbf{A}$ and $\mathbf{B}$, linear fits to the whole logarithmic dataset for the FRP and SRP were made (continuous grey lines, slopes indicated). 

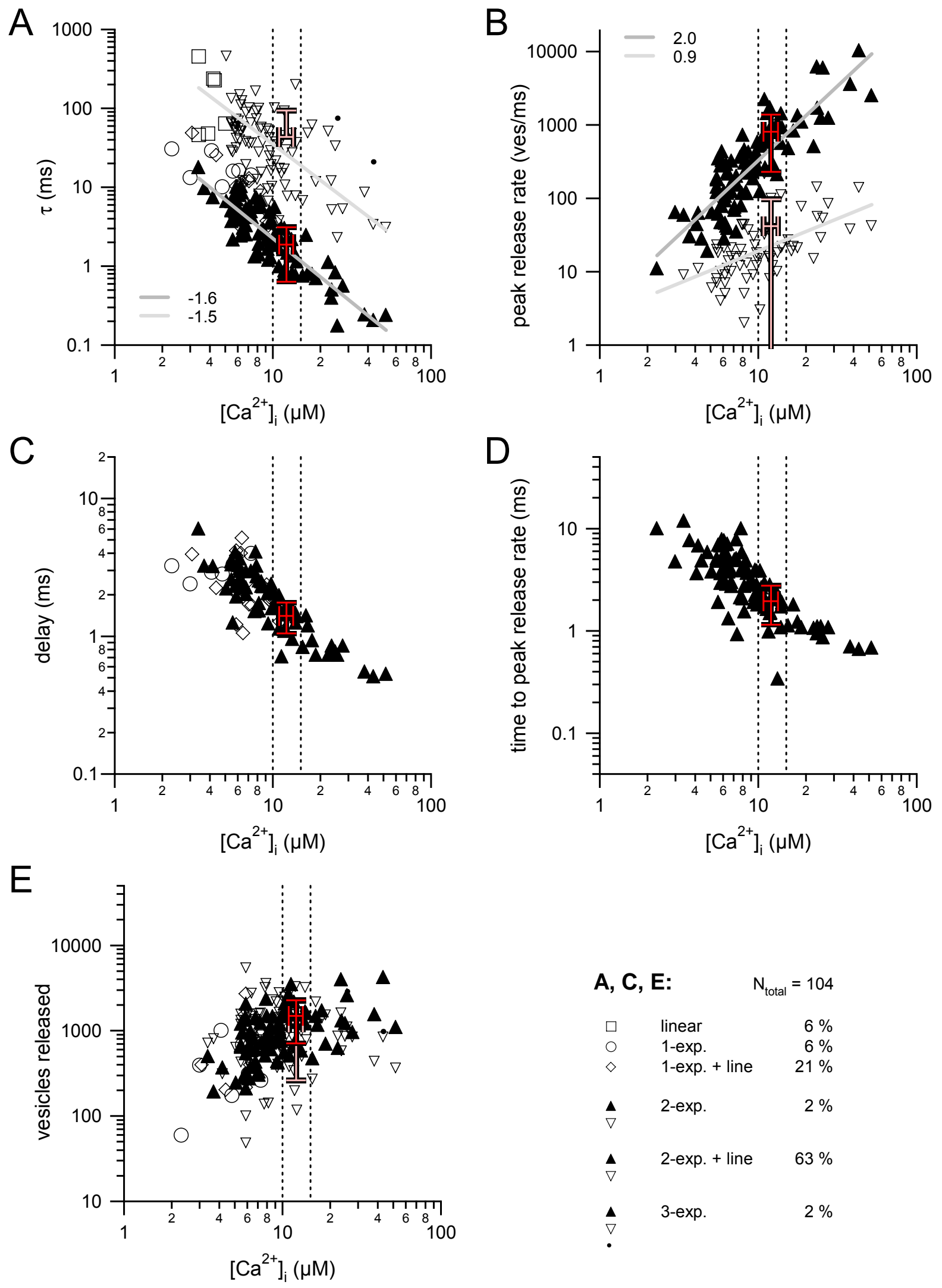
Summarizing data from several cells over a wide range of stimulation intensities (Fig. 14, N= 104) unveiled a clear appearance of a second release component in the time course of cumulative release (Fig. 14A, B, E; Fig. 15B), especially for $\left[\mathrm{Ca}^{2+}\right]_{i}$ above $5 \mu \mathrm{M}$. Between 2 $\mu \mathrm{M}$ and $60 \mu \mathrm{M}\left[\mathrm{Ca}^{2+}\right]_{\mathrm{i}}$, the majority of responses could be classified as '2-exponential + line' $(63 \%)$. '1-exponential + line' time courses were frequent $(21 \%)$, but were observed only in the low $\left[\mathrm{Ca}^{2+}\right]_{\mathrm{i}}$ range of less than $11 \mu \mathrm{M} .6 \%$ of all responses were classified '1-exponential' and appeared only below $8 \mu \mathrm{M}, 6 \%$ were 'linear' only occurring below $5 \mu \mathrm{M}, 2 \%$ were '2exponential', and $2 \%$ '3-exponential'. Thus, from this analysis two kinetically distinct components of release were also evident for a spatially homogeneous stimulus, comparable to those observed after presynaptic depolarizations (Figs. $10-11$ ). A differentiation between an FRP and an SRP was likely to be based on intrinsically different $\mathrm{Ca}^{2+}$ sensitivities, assuming that refilling of a single, fast release component could not account for the appearance of the slow release component (see chapter 4.2).

For a $\left[\mathrm{Ca}^{2+}\right]_{\mathrm{i}}$ range of $10-15 \mu \mathrm{M}$, the estimated release time constants of the FRP and SRP were $1.9 \pm 1.2 \mathrm{~ms}$ and $43.4 \pm 50.0 \mathrm{~ms}$ at $12.2 \pm 1.2 \mu \mathrm{M}\left[\mathrm{Ca}^{2+}\right]_{\text {i }}$, respectively $(\mathrm{N}=18, \mathrm{p}=$ 0.0025; Fig. 14A, red error bars; results from all responses with two release components were averaged). The $\left[\mathrm{Ca}^{2+}\right]_{i}$ range of $10-15 \mu \mathrm{M}$ was chosen, because in a $\mathrm{Ca}^{2+}$ microdomain after a single AP about the same local peak $\left[\mathrm{Ca}^{2+}\right]_{i}$ is reached (Schneggenburger and Neher, 2000; Bollmann et al., 2000). In the same range of stimulation intensities, the estimated pool sizes had comparable amplitudes of $1491 \pm 778$ vesicles for the FRP, and $1255 \pm 992$ vesicles for the SRP (Fig. 14E, red error bars). While the peak release rate was solely determined by the fast release component (e.g. in Fig. 14A, 14B), and therefore can be directly quantified for the FRP, the peak release rate of the SRP was extracted indirectly from the time course of cumulative release (chapter 2.4, and Fig. 12C, dashed green lines). As for the time constants of release, the estimates for peak release rates (Fig. 14B) differed significantly between the FRP and the SRP, ranging on average between $806 \pm 577$ ves/ms and $41 \pm 55$ ves/ms for a $\left[\mathrm{Ca}^{2+}\right]_{i}$ range of $10-15 \mu \mathrm{M}$, respectively ( $p=0.00003$; Fig. 14B, red error bars). Delays, as estimated by the zerocrossing of the regressions fitted to the time course of cumulative release, and time-to-peak release rates could be specified for the FRP only. In the given range of $10 \mu \mathrm{M}-15 \mu \mathrm{M}\left[\mathrm{Ca}^{2+}\right]_{\mathrm{i}}$, fast fusion already started with a delay of $1.4 \pm 0.4 \mathrm{~ms}$, and peak release rates were reached after $2.0 \pm 0.8 \mathrm{~ms}$, comparing well to previous estimates (Schneggenburger and Neher, 2000; Bollmann et al., 2000; Wölfel and Schneggenburger, 2003). 


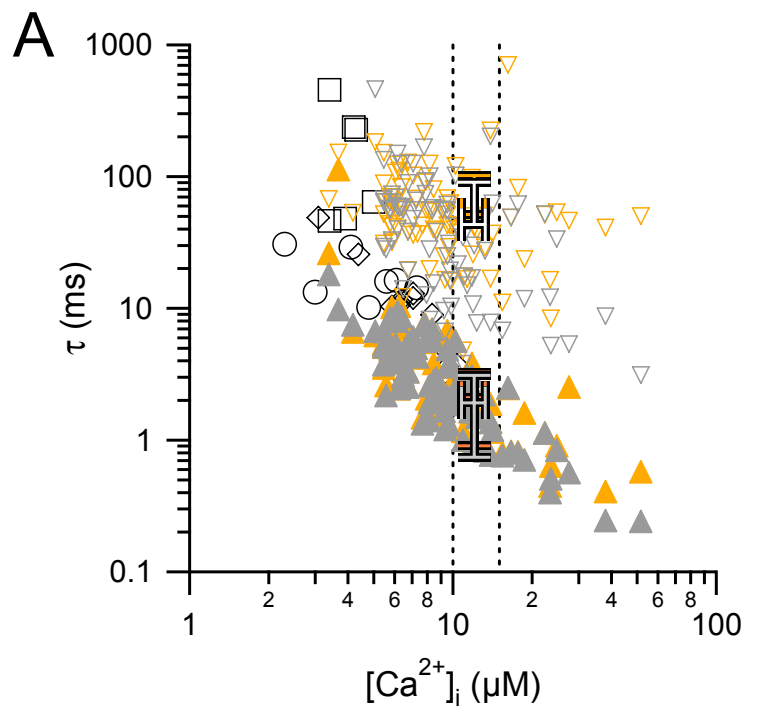

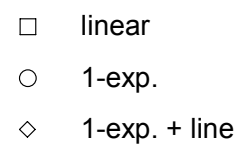

all others plotted twice as:

$\triangle \quad$ 2-exp., fast component 2-exp., slow component

$\Delta \quad$ 2-exp. + line, fast component 2-exp. + line, slow component

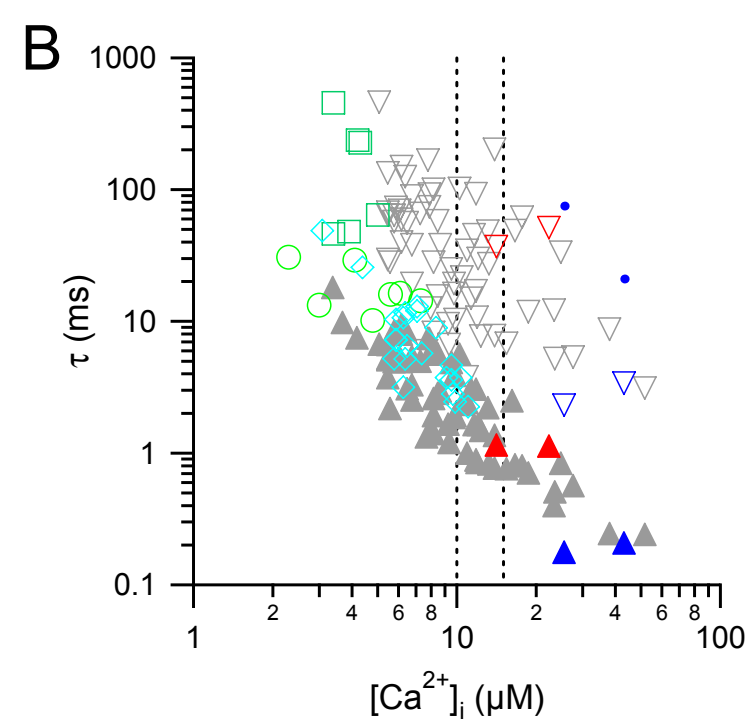

$\square \quad$ linear
$\quad$ 1-exp.
$\Delta \quad$ 1-exp. + line
$\Delta \quad$ 2-exp., fast component
$\nabla \quad$ 2-exp., slow component
$\Delta \quad$ 2-exp. + line, fast component
$\nabla \quad$ 2-exp. + line, slow component
$\Delta \quad$ 3-exp., fast component
$\nabla \quad$ 3-exp., slow component
- $\quad$ 3-exp., slowest component

Fig. 15 Comparison of release time constants approximated by different regressions to the time course of cumulative release

A All results from time courses of cumulative release with at least two exponential components (same as triangles in Fig. 14A) are displayed twice, with time constants of release estimated by either '2-exponential' (yellow symbols), or '2-exponential + line' fits (grey symbols; results from release with only a single component are shown in black). Between $10 \mu \mathrm{M}$ to $15 \mu \mathrm{M}\left[\mathrm{Ca}^{2+}\right]_{\mathrm{i}}$, '2exponential' fits yielded release time constants for the FRP (closed triangles) and SRP (open triangles) of $2.1 \pm 1.1 \mathrm{~ms}$ and $51.5 \pm 50.6 \mathrm{~ms}$ on average, respectively (yellow error bars). '2exponential + line' fits yielded $1.9 \pm 1.2 \mathrm{~ms}$ and $43.2 \pm 47.6$ for the FRP and SRP, respectively (grey error bars). B The final classification of release kinetics is illustrated by different symbols and colors, as indicated below the image. 
Both, the fast and the slow release component, showed a steep $\left[\mathrm{Ca}^{2+}\right]_{i}$ dependence. In the double-logarithmic plot of time constants as a function $\left[\mathrm{Ca}^{2+}\right]_{\mathrm{i}}$, linear regressions yielded a power relationship of -1.6 and -1.5 for the FRP and SRP, respectively (Fig. 14A, continuous grey lines). Fitting peak release rates in the same way resulted in power relationships of 2.0 and 0.9 for FRP and SRP, respectively (Fig. 14B, continuous grey lines), indicating the strong $\mathrm{Ca}^{2+}$ dependence of release for both, FRP and SRP. The observed $\left[\mathrm{Ca}^{2+}\right]_{\mathrm{i}}$ dependence of the FRP was lower than previous estimates obtained with EPSC recordings (4.2 in Schneggenburger and Neher, 2000; 4.4 in Bollmann et al., 2000), or presynaptic $C_{m}$ measurements (4 in Wölfel and Schneggenburger, 2003). However, here the power relationship resulted from fitting in a range of $3 \mu \mathrm{M}$ to $50 \mu \mathrm{M}\left[\mathrm{Ca}^{2+}\right]_{\mathrm{i}}$, whereas in the other studies for a maximal estimate a range below $8 \mu \mathrm{M}$ was chosen, and the $\left[\mathrm{Ca}^{2+}\right]_{i}$ dependence of release rates at $\left[\mathrm{Ca}^{2+}\right]_{\mathrm{i}}$ above $8 \mu \mathrm{M}$ was lower, as compared to estimates below $8 \mu \mathrm{M}$ (Schneggenburger and Neher, 2000; 4.4 in Bollmann et al., 2000; Wölfel and Schneggenburger, 2003).

To demonstrate that the results were not a consequence of the classification which fit was defined to describe the release time course best (see Methods, chapter 2.4.2; Fig. 14), results from '2-exponential + line' regressions were compared to those from '2-exponential' fits (Fig. 15A). All release time constants from $\mathrm{Ca}^{2+}$ uncaging responses with at least two exponential components (Fig. 14A, triangles), were plotted again in Fig. 15A, but now always taking results from the corresponding '2-exponential + line' fits (grey triangles). This data set was then plotted again, this time always taking results from the corresponding '2-exponential' fits (yellow triangles). Essentially, both regression types, '2-exponential + line' and '2exponential', yielded very similar release time constants (Fig. 15A) and amounts of cumulative release (not shown) for the FRP, and below $15 \mu \mathrm{M}\left[\mathrm{Ca}^{2+}\right]_{\mathrm{i}}$, also for the SRP. Between $10 \mu \mathrm{M}$ and $15 \mu \mathrm{M}\left[\mathrm{Ca}^{2+}\right]_{\mathrm{i}}$, the '2-exponential + line' fits yielded averages of $1.9 \pm 1.2$ ms with $1470 \pm 782$ vesicles, and $43.2 \pm 47.6$ ms with $1329 \pm 1021$ vesicles for the FRP and SRP, respectively. The corresponding estimates from the '2-exponential' fits were $2.1 \pm 1.1$ ms with $1567 \pm 866$ vesicles for the FRP, and $51.5 \pm 50.6$ ms with $1396 \pm 1132$ vesicles for the SRP. The differences between the two classes of regressions were not significant, neither regarding time constants (FRP: $p=0.65$; SRP: $p=0.62$ ) nor cumulative release estimates (FRP: $p=0.73$; SRP: $p=0.85$ ).

However, at $\left[\mathrm{Ca}^{2+}\right]_{\mathrm{i}}$ above $15 \mu \mathrm{M}$, the time constant estimates for the SRP seemed to differ, comparing results from '2-exponential + line' fits to those of '2-exponential' fits (Fig. 15A, open grey and yellow triangles, respectively). Those from '2-exponential' fits showed a 
tendency towards slower kinetics, for the FRP as well as for the SRP. This can be explained by a more pronounced segregation of SRP release rates and rates of SRP refilling. As a result, refilling of the SRP should have appeared as an additional release component, forcing a simple '2-exponential' regression to intermediate estimates between release kinetics of the FRP, the SRP, and the SRP being refilled. This effect should be avoidable by defining the '2exponential + line' regression to be the better approximation, as was done for the final dataset and further analysis (see Methods, chapter 2.4.2).

The whole dataset after the final classification of release time courses was plotted again for clarity. Fig. 15B shows the same data as Fig. 14A, but here, release time constants of responses with at least two exponential components (Fig. 14A, triangles) were discriminated by different colors (Fig. 15B, see legend).

\subsection{Selective depletion of the FRP by short presynaptic depolarizations}

The results described before suggest that $\mathrm{Ca}^{2+}$ uncaging leads to two separable phases of release from the RRP: the FRP and the SRP. Thus, intrinsic heterogeneity in $\mathrm{Ca}^{2+}$ sensitivity was likely to be present in this CNS synapse.

However, the ability to estimate SRP release kinetics was limited, because the SRP became prominent in the later phase of release, being masked by already ongoing release from the FRP. To work out the SRP kinetics in more detail, a cross-depletion experiment was designed. A short depolarization aimed at complete depletion of the FRP, while leaving a major part of the SRP unreleased. This short depolarization would have to be long enough to release all FRP vesicles, but short enough to leave a substantial part of the SRP unreleased. Then, for a subsequent second stimulus, these remaining SRP vesicles should release in separation from the FRP, disclosing their kinetics properties without any masking by the FRP.

\subsubsection{Pre-depletion of the FRP followed by $\mathrm{Ca}^{2+}$ uncaging}

For a detailed examination of SRP release kinetics in separation, a short depolarization to pre-deplete the FRP was followed by a $\mathrm{Ca}^{2+}$ uncaging stimulus. To release all FRP vesicles, the depolarization had to last at least three times longer than the FRP depletion time 
constant of about 2 to $3 \mathrm{~ms}$ (Figs. 10E, 14A). On the other hand, to leave a larger fraction of the SRP unreleased (here more than $70 \%$ ), the depolarization had to last at least three times shorter than the SRP depletion time constant of about 40 ms (Figs. 10E, 14A). Thus, a depolarization of 8 ms length should dump out vesicles from the FRP, while only a minor part of the SRP would be released before the end of the depolarizing stimulus. Since refilling of the FRP had a time constant of several seconds (Sakaba and Neher, 2001b), inter-stimulus intervals of less than $50 \mathrm{~ms}$ should lead to less than $5 \%$ refilling of the FRP.

In the two examples shown (Figs. 16, 17), such an experimental approach was tested. Each example includes only data from an individual cell, and several stimulus protocols were applied to each cell.

To the first cell (Fig. 16), three different stimulus protocols were applied: a $\mathrm{Ca}^{2+}$ uncaging stimulus followed by a $50 \mathrm{~ms}$ depolarization to test pool depletion (black trace), a $\mathrm{Ca}^{2+}$ uncaging stimulus preceded by a $8 \mathrm{~ms}$ depolarization (red trace), and a $50 \mathrm{~ms}$ depolarization alone (dashed blue trace). All cumulative release responses after $\mathrm{Ca}^{2+}$ uncaging were classified as '2-exponential + line', and the unconditioned $\mathrm{Ca}^{2+}$ uncaging stimulus to $11 \mu \mathrm{M}$ $\left[\mathrm{Ca}^{2+}\right]_{\mathrm{i}}$ (Fig. 16, black trace) induced release of 3158 and 2342 vesicles from the FRP with a time constants of $3.0 \mathrm{~ms}$ (dotted black trace). During a cross-depletion stimulation protocol in the same cell (Fig. 16, red traces) the conditioning $8 \mathrm{~ms}$ pre-depolarization to $0 \mathrm{mV}$ released 3260 vesicles fast (dotted red line), but there was almost no late release. The effect of stimulus cessation became more obvious when comparing the EPSC decay to that of the prolonged depolarization (Fig. 16, compare red trace to dashed blue trace, see arrowheads). Both EPSCs started at the same relative time, passed through the same peak, and initially showed the same speed of decay. But stimulus cessation led to an acceleration of the EPSC decay, so while depletion of the FRP seemed to be complete after the $8 \mathrm{~ms}$ predepolarization, as compared to control (3260 and 3158 vesicles, respectively), slow vesicles should have mainly remained unreleased. Indeed, after the pre-depolarization, fusion competent vesicles were still left for the subsequent $\mathrm{Ca}^{2+}$ uncaging stimulus, however, here again release could be separated into a fast and a slow component, with a fast fusion time constant of $3.9 \mathrm{~ms}$, similar to that for the FRP. Thus, despite the strong apparent depletion efficiency of the pre-depolarization that led to the same amount of release from the FRP as the control stimulus, there were another 1655 vesicles left being rapidly released by the subsequent $\mathrm{Ca}^{2+}$ uncaging stimulus (Fig. 16, red asterisk). In total, the cross-depletion protocol induced fusion of approximately 4915 FRP vesicles, while the control stimulus released only $64 \%$ of this amount. This was unexpected, because, as mentioned above, such a strong contribution by FRP refilling within 50 ms was rather unlikely. This discrepancy 


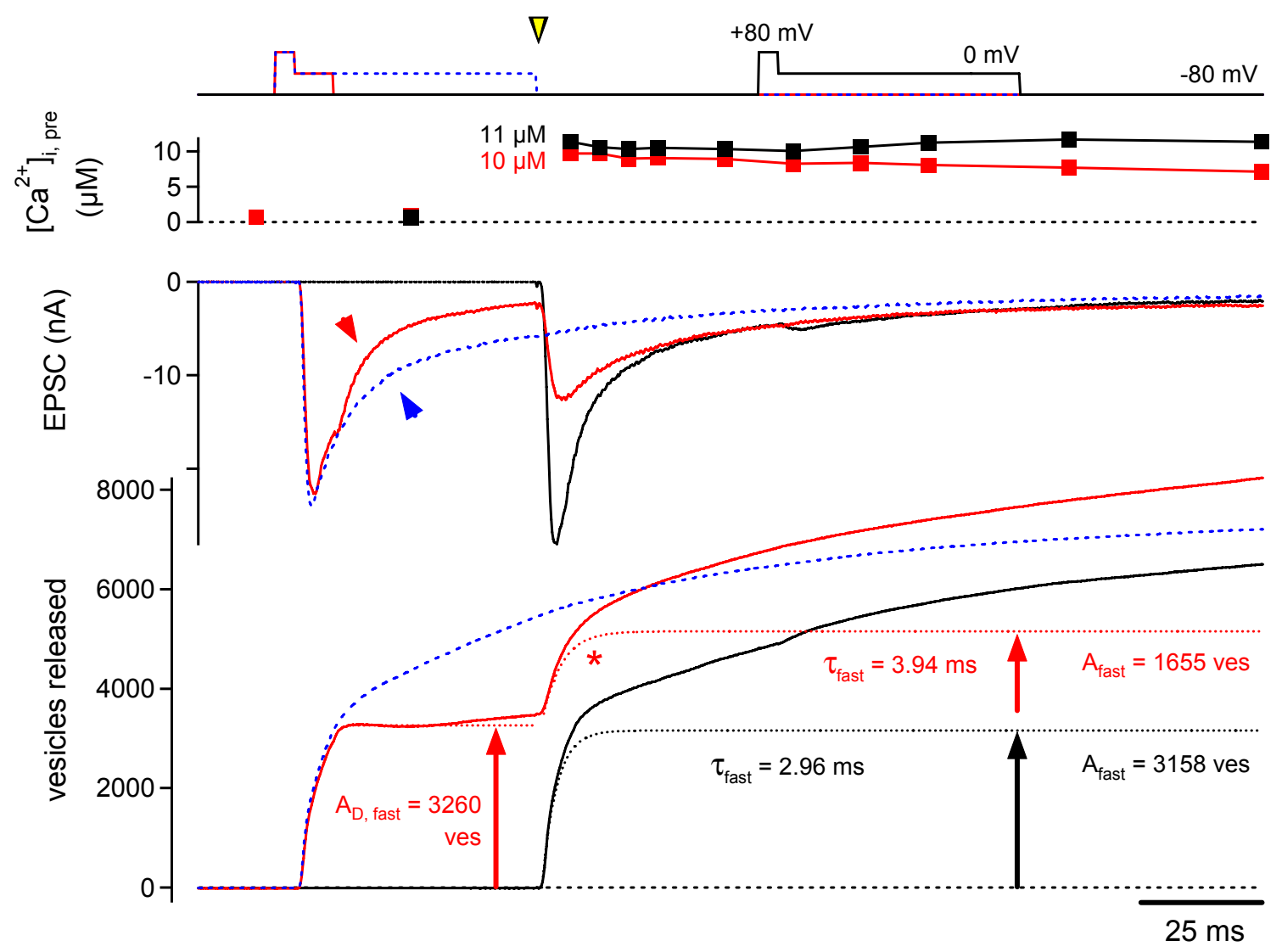

Fig. 16 Cross-depletion experiment to selectively pre-deplete the FRP before subsequent $\mathrm{Ca}^{2+}$ uncaging (example cell 1)

A selective pre-depletion of the FRP to leave only SRP vesicles remaining for subsequent $\mathrm{Ca}^{2+}$ uncaging (yellow arrowhead) was aimed. Three different stimulus protocols (top panel) have been applied in the same cell: a control $\mathrm{Ca}^{2+}$ uncaging stimulus (black traces to $11 \mu \mathrm{M}\left[\mathrm{Ca}^{2+}\right]$, followed by a $50 \mathrm{~ms}$ depolarization, ISI $=50 \mathrm{~ms}$ ), a cross-depletion protocol with a $8 \mathrm{~ms}$ depolarization to $0 \mathrm{mV}$ (after $4 \mathrm{~ms}$ at $+80 \mathrm{mV}$ ) followed by flash photolysis (red traces to $10 \mu \mathrm{M}$; $I S \mid=42 \mathrm{~ms}$ ), and a single $50 \mathrm{~ms}$ depolarization (dashed blue traces). For the $\mathrm{Ca}^{2+}$ uncaging responses and the pre-depolarization, contributions of the FRP are indicated (dotted curves), arrows mark the amounts of release. Please note that the EPSC maxima for the black, red, and blue traces were subject to amplifier saturation. The temporal order of stimulation was: red, blue, black. 
in the size estimates of the FRP after unconditioned $\mathrm{Ca}^{2+}$ uncaging to $8 \mu \mathrm{M}\left[\mathrm{Ca}^{2+}\right]_{\mathrm{i}}$ indicated a dependence of FRP size estimates on stimulus intensities (see below and chapter 3.4).

The incomplete depletion of the FRP by a pre-depolarization (Fig. 16, red trace) can neither be explained by pure intrinsic nor by pure positional heterogeneity. In the case of pure intrinsic heterogeneity, the consistent FRP size estimates from the pre-depolarization (Fig. 16 , red trace) compared to the unconditioned $\mathrm{Ca}^{2+}$ uncaging stimulus (black trace) would be reasonable. However, for intrinsic heterogeneity, the depolarization of $8 \mathrm{~ms}$ should have led to complete depletion of the FRP so that no fast vesicles would have remained for the subsequent $\mathrm{Ca}^{2+}$ uncaging stimulus. In the case of pure positional heterogeneity, FRP predepletion by a depolarization could indeed have been incomplete, because only FRP vesicles close to the voltage activated $\mathrm{Ca}^{2+}$ channels would have been released by the depolarization, but FRP vesicles located further away would have remained fusioncompetent for the subsequent $\mathrm{Ca}^{2+}$ uncaging stimulus. But on the other hand, positional heterogeneity does not account for the smaller FRP size estimated after unconditioned $\mathrm{Ca}^{2+}$ uncaging (Fig. 16, black traces; $A_{\text {fast }}=3158$ vesicles), as compared to the cross-depletion result with a comparable $\mathrm{Ca}^{2+}$ uncaging stimulus intensity (red traces, summed $\mathrm{A}_{\text {fast }}=4915$ vesicles). However, in this example (Fig. 16), amplifier saturation affected peak EPSCs, and therefore, the observations were compared to results from other cells, as follows.

In other cells with no amplifier saturation, the same phenomenon could be observed, as shown in Fig. 17. Here, the cross-depletion protocol with a $8 \mathrm{~ms}$ pre-depolarization led to release of 1405 FRP vesicles followed by another 319 FRP vesicles due to subsequent $\mathrm{Ca}^{2+}$ uncaging, being 1724 FRP vesicles in total (Fig. 17, red traces). The unconditioned $\mathrm{Ca}^{2+}$ uncaging stimulus released only 1380 FRP vesicles (black traces), but due to the large peak EPSC amplitude in this case, postsynaptic AMAP-receptor saturation could not be excluded. Please note, that in this example the pre-depolarizations did not release FRP vesicles selectively, but a substantial contribution to release also came from the SRP (red arrowhead). The phenomenon of enlarged release from the FRP during cross-depletion stimulation could be observed frequently, also when the length of the pre-depolarization was varied between 4 $\mathrm{ms}$ and $32 \mathrm{~ms}$, and the inter-stimulus interval was reduced to $25 \mathrm{~ms}$ (not shown).

Furthermore, pre-depolarizations led to a slowing of the FRP release time constant. As shown in Fig. 17, the control $\mathrm{Ca}^{2+}$ uncaging stimulus to $13 \mu \mathrm{M}\left[\mathrm{Ca}^{2+}\right]_{\mathrm{i}}$ induced release from the FRP with a time constant of $0.8 \mathrm{~ms}$ (black traces). But for the $\mathrm{Ca}^{2+}$ uncaging stimulus 


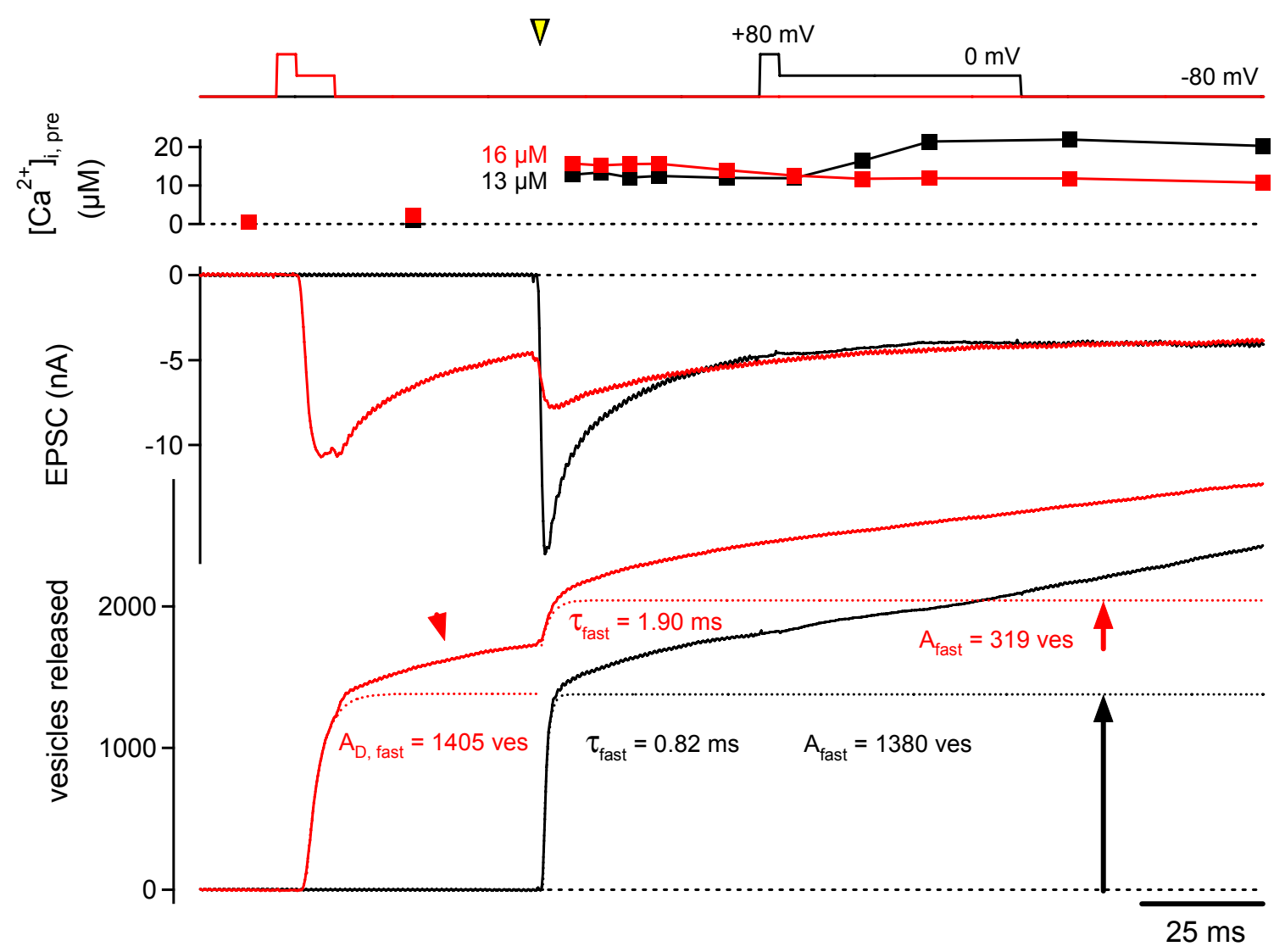

Fig. 17 Cross-depletion experiment to selectively pre-deplete the FRP before subsequent $\mathrm{Ca}^{2+}$ uncaging (example cell 2)

The same experimental approach as in figure 16 was performed in a different cell. Here, only two different stimulus protocols have been applied: a control $\mathrm{Ca}^{2+}$ uncaging stimulus (black traces to $13 \mu \mathrm{M}\left[\mathrm{Ca}^{2+}\right]_{\text {i }}$, followed by a $50 \mathrm{~ms}$ depolarization, ISI = $\left.50 \mathrm{~ms}\right)$, and a cross-depletion stimulus with a $8 \mathrm{~ms}$ depolarization to $0 \mathrm{mV}$ (after $4 \mathrm{~ms}$ at $+80 \mathrm{mV}$ ) followed by $\mathrm{Ca}^{2+}$ uncaging to $16 \mu \mathrm{M}\left[\mathrm{Ca}^{2+}\right]_{\mathrm{i}}$ (red traces; ISI = $42 \mathrm{~ms}$ ). The temporal order of stimulation was: black, red. 
after pre-stimulation, the FRP release time constant increased about twofold to $1.9 \mathrm{~ms}$ (Fig. 17, red traces), even though stimulus intensity was higher $\left(\left[\mathrm{Ca}^{2+}\right]_{i}=16 \mu \mathrm{M}\right)$. This phenomenon was also observed in the other cell shown (Fig. 16), where pre-stimulation led to an increase in the FRP release time constant from $3.0 \mathrm{~ms}$ to $3.9 \mathrm{~ms}$ at $11 \mu \mathrm{M}$ and $10 \mu \mathrm{M}$ $\left[\mathrm{Ca}^{2+}\right]_{\mathrm{i}}$, respectively.

The results from all cells tested were pooled and analyzed, and the time constants and release amount estimates from $\mathrm{Ca}^{2+}$ uncaging stimuli conditioned by pre-depolarizations $(8-$ $16 \mathrm{~ms}$ ) were compared to those of unconditioned control $\mathrm{Ca}^{2+}$ uncaging stimuli (Fig. 18; $\mathrm{N}=$ 50 and $\mathrm{N}=104$, respectively). Again, most of the cumulative release time-courses were classified as '2-exponential + line' (58\%, $63 \%$ in control), but now the pre-depolarization increased the contribution of '1-exponential + line' responses almost two-fold from $21 \%$ in control to $38 \%$. Neither a '3-exponential', nor a 'linear' time course, and only 1 example with a '2-exponential' and another one with a '1-exponential' time course could be observed.

Fig $18 \mathrm{~A}$ shows the release time constants for $\mathrm{Ca}^{2+}$ uncaging-induced release following partial pool depletion (Fig. 18A, red symbols; $\mathrm{N}=50$ ). For comparison, the $\left[\mathrm{Ca}^{2+}\right]_{\mathrm{i}}$ dependence of unconditioned release (black symbols, replotted from Fig. 18A; $N=104$ ) is also displayed. As can be seen, the time constants of $\mathrm{Ca}^{2+}$ uncaging-induced release following partial pool depletion were similar to control (Fig. 18A, red and black symbols, respectively).

In the range of $10 \mu \mathrm{M}$ to $15 \mu \mathrm{M}\left[\mathrm{Ca}^{2+}\right]_{\text {, }}$, on average the FRP released with a time constant of $3.1 \pm 1.4 \mathrm{~ms}$ (Fig. $18 \mathrm{~A}$, closed red triangles; $\mathrm{N}=16$; $\left[\mathrm{Ca}^{2+}\right]_{\mathrm{i}}=11.7 \pm 1.3 \mu \mathrm{M}$ ). Thus, after predepolarizations, fast release became slower as compared to $1.9 \pm 1.2 \mathrm{~ms}$ in control (closed black triangles; $\left.N=18 ;\left[\mathrm{Ca}^{2+}\right]_{i}=12.2 \pm 1.2 \mu \mathrm{M} ; \mathrm{p}=0.016\right)$. Also, a decrease in the peak release rate of the fast release component could be observed (Fig. 18B), declining from 806 $\pm 577 \mathrm{ves} / \mathrm{ms}$ in control to $171 \pm 153 \mathrm{ves} / \mathrm{ms}$ after pre-stimulation $(p=0.00022$; closed red and black triangles, respectively). This was expected, because of a reduction in available FRP vesicles due to pre-stimulation. If this decrease in peak release rates either originated only from a reduction in the number of fusion-competent vesicles, or resulted also from a deceleration of release kinetics, is not directly conclusive, but the observed slowing of the FRP release time constant (Fig. 18A) indicates that deceleration of release contributed to the reduction in the FRP peak release rate. 

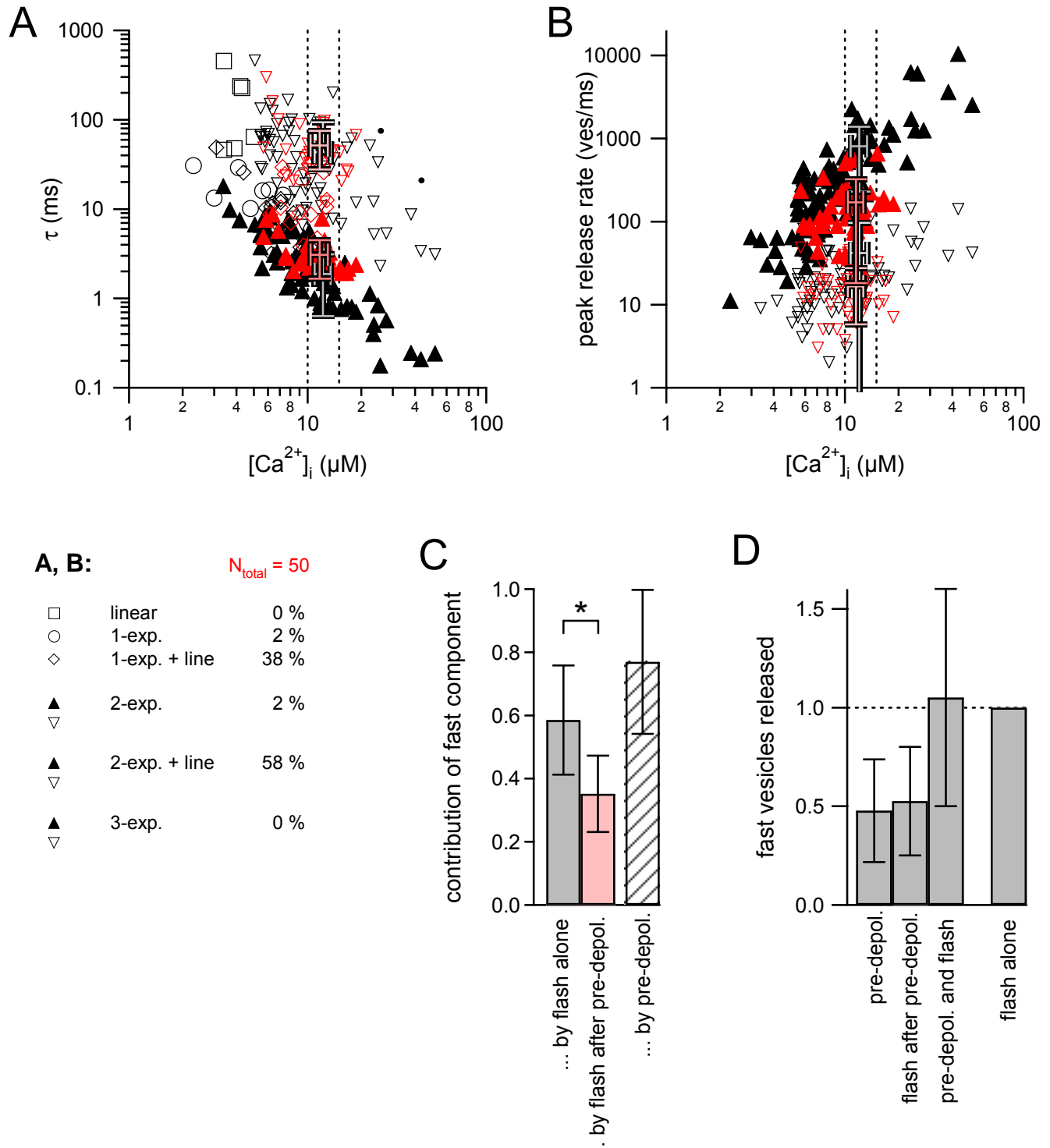

Fig. 18 Cross-depletion experiments to separate the SRP from the RRP before subsequent $\mathrm{Ca}^{2+}$ uncaging

The effects of pre-depolarizations on kinetics of release during a subsequent $\mathrm{Ca}^{2+}$ uncaging stimulus are shown. Release time constants $(A)$, and peak release rates $(B ; N=50)$ are displayed. In the range of $10 \mu \mathrm{M}$ to $15 \mu \mathrm{M}\left[\mathrm{Ca}^{2+}\right]_{\mathrm{i}}, \mathrm{Ca}^{2+}$ uncaging with $8 \mathrm{~ms}$ pre-depolarizations induced time constants of $3.1 \pm 1.4 \mathrm{~ms}(51.5 \pm 23.7 \mathrm{~ms})$ and peak release rates of $171 \pm 153$ ves/ms (17 $\pm 11 \mathrm{ves} / \mathrm{ms})$ for the FRP (SRP) at $11.7 \pm 1.3 \mu \mathrm{M}\left[\mathrm{Ca}^{2+}\right]_{i}$ (red symbols; $\mathrm{N}=16$ ), whereas control data yielded time constants of $1.9 \pm 1.2 \mathrm{~ms}(43.4 \pm 50.0 \mathrm{~ms})$ and peak release rates of $806 \pm 577 \mathrm{ves} / \mathrm{ms}(42 \pm 55 \mathrm{ves} / \mathrm{ms})$ for the FRP (SRP) at $12.2 \pm 1.2 \mu \mathrm{M}\left[\mathrm{Ca}^{2+}\right]_{\mathrm{i}}$ (black symbols as in Fig. 14A, B; $N=18)$. C The contribution of fast release during each stimulus was reduced from $59 \pm 17 \%$ in control (left column) to $35 \pm 12 \%$ due to pre-depolarizations (middle column). During the pre-depolarizations, $80 \pm 23 \%$ of release showed fast kinetics (right column). D Amounts of cumulative release from the FRP during the whole cross-depletion stimulation were normalized to the amount during a control $\mathrm{Ca}^{2+}$ uncaging stimulus within the same cell. Pre-depolarizations led to release of $48 \pm 26 \%$ of the FRP, and subsequent $\mathrm{Ca}^{2+}$ uncaging released another $53 \pm 28 \%$ of the FRP, so that totally $105 \pm 55 \%$ of the FRP were released, as compared to control (rightmost column; $N=4$ ). 
However, release from the SRP with an averaged time constant of $51.5 \pm 23.7 \mathrm{~ms}$ did not differ significantly from $43.4 \pm 50.0 \mathrm{~ms}$ in control ( $p=0.57$; Fig. $18 \mathrm{~A})$, but the data scattered widely (open red and black triangles, respectively). The peak release rate of the SRP changed from $42 \pm 55 \mathrm{ves} / \mathrm{ms}$ in control to $17 \pm 11 \mathrm{ves} / \mathrm{ms}$ ( $p=0.088$; Fig. 18B).

The amount of remaining fusion-competent vesicles was reduced for both, FRP and SRP, due to the pre-depolarization as compared to control (data not shown). In the $\left[\mathrm{Ca}^{2+}\right]_{\mathrm{i}}$ range of $10-15 \mu \mathrm{M}$, the amount of available FRP vesicles decreased from $1491 \pm 778$ vesicles in control to $554 \pm 500$ vesicles after pre-depolarization, for the SRP from $1255 \pm 992$ vesicles to $941 \pm 632$ vesicles. The finding of incomplete FRP depletion during pre-depolarizations, as seen in the example traces before (Figs. 16, 17), becomes visible when normalizing the data in the range of $10 \mu \mathrm{M}-15 \mu \mathrm{M}\left[\mathrm{Ca}^{2+}\right]_{\mathrm{i}}$. This is shown in Fig. $18 \mathrm{C}$. In a control stimulus, i.e. $\mathrm{Ca}^{2+}$ uncaging without pre-depolarization, $59 \pm 17 \%$ of all vesicles released had fast kinetics, while the rest was slow (left column). But after a conditioning pre-depolarization, the FRP contributed only by $35 \pm 12 \%$ when giving a subsequent $\mathrm{Ca}^{2+}$ uncaging stimulus (mid column). Thus, the contribution of the FRP decreased significantly ( $p=0.0012$ ), but not nearly as much as was expected. A complete pre-depletion of the FRP by pre-depolarization was not achieved, even though release due to pre-depolarization consisted to $80 \pm 23 \%$ of fast vesicles (Fig. 18C, right column).

This incomplete pre-depletion of the FRP can be explained by an insufficient potency of depolarizations under the present experimental conditions. Fig. 18D shows the averaged amount of release from the FRP during cross-depletion stimulation (three leftmost columns), normalized to control as obtained by a single, unconditioned $\mathrm{Ca}^{2+}$ uncaging stimulus within the same cell (rightmost column). On average, during cross-depletion stimulation a predepolarization released $48 \pm 26 \%$ of the FRP (Fig. 18D, leftmost column), thus, the predepolarization could not efficiently release all vesicles from the FRP. Therefore, for a subsequent $\mathrm{Ca}^{2+}$ uncaging stimulus a substantial amount of fast vesicles was still available (Fig. 18C, mid column).

The efficiency in inducing FRP release during the whole cross-depletion cycle was also compared to control (Fig. 18D). A pre-depolarization released on average $48 \pm 26 \%$ of the FRP (Fig. 18D, leftmost column), as compared to a single $\mathrm{Ca}^{2+}$ uncaging to $10-15 \mu \mathrm{M}$ $\left[\mathrm{Ca}^{2+}\right]_{i}$ within the same cell (rightmost column). The subsequent $\mathrm{Ca}^{2+}$ uncaging stimulus, elevating $\left[\mathrm{Ca}^{2+}\right]_{\mathrm{i}}$ to $10-15 \mu \mathrm{M}$, triggered release of another $53 \pm 28 \%$ ( ${ }^{\text {nd }}$ column from left), so that the whole cross-depletion protocol led to fusion of $105 \pm 55 \%$ of FRP vesicles ( $3^{\text {rd }}$ column from left), as compared to control (rightmost column). This averaged result is opposed to the findings from the example traces shown before (Figs. 16, 17), which 
suggested that the sum of FRP vesicles released in a cross-depletion approach is bigger than the number of FRP vesicles released in control. However, the standard deviation for averaged estimates of total release from the FRP during the whole cross-depletion stimulation is large, and furthermore, averaging data might have masked effects apparent only in a subset of the data. Thus, here a quantitative conclusion can hardly be made.

It seems that a short pulse did not fully deplete the FRP, opposite to what was expected when designing the cross-depletion approach. Instead, if $\mathrm{Ca}^{2+}$ influx through $\mathrm{Ca}^{2+}$ channels was stopped after a few milliseconds, a time at which the FRP should have been depleted, a remaining fast component showed up at the subsequent $\mathrm{Ca}^{2+}$ uncaging stimulus (Fig. 16 17, red traces). Since the time interval between the end of the depolarization, and subsequent $\mathrm{Ca}^{2+}$ uncaging (42 - $34 \mathrm{~ms}$ ) was too short to allow for substantial recovery of FRP vesicles, assumingly FRP vesicles were left unreleased after a short (8 - $16 \mathrm{~ms})$ depolarization. This indicates a contribution of positional heterogeneity, at least, for the fast release component.

\subsubsection{Pre-depletion of the FRP in double-depolarization experiments}

The weak efficiency of pre-depolarizations in depleting the FRP was possibly due to strong $\mathrm{Ca}^{2+}$ buffering in the presynaptic terminal. High concentrations of the $\mathrm{Ca}^{2+}$ chelator might have led to a potent buffering of $\mathrm{Ca}^{2+}$ influx through the depolarization activated $\mathrm{Ca}^{2+}$ channels, although $\mathrm{Ca}^{2+}$ chelator preloading was high when mixing the intracellular presynaptic solution (about $90 \%$; see also methods, chapter 2.3). More intense local restriction of $\mathrm{Ca}^{2+}$ microdomains by stronger $\mathrm{Ca}^{2+}$ buffering was possibly counterbalanced by prolonged depolarizations ( $8-16 \mathrm{~ms}$, see chapter 3.3.1). But still, slower diffusion of $\mathrm{Ca}^{2+}$ to more distant vesicles due to stronger $\mathrm{Ca}^{2+}$ buffering might have prevented complete depletion of the fast release component during pre-depolarizations. Thus, a decreased release efficiency of depolarizations might have enhanced effects of positional heterogeneity on release kinetics. As a consequence, an additional effect by positional heterogeneity mixing with intrinsic heterogeneity could explain the still large contribution of fast vesicles to release at $\mathrm{Ca}^{2+}$ uncaging stimuli after pre-depolarization (35 $\pm 12 \%$; Fig. 18C, red column). For a more conclusive description, the approach was repeated in a slightly different manner, as follows.

Similar to the cross-depletion experiments using depolarizations and subsequent $\mathrm{Ca}^{2+}$ uncaging stimuli (chapter 3.3.1), double-pulse depolarizations aimed to pre-deplete the FRP 
with the first stimulus and then to look at release from the SRP in separation during the second pulse. Additionally to changing the second pulse, now being a depolarization in place of a $\mathrm{Ca}^{2+}$ uncaging stimulus, also the $\mathrm{Ca}^{2+}$ buffering conditions were changed. No $\mathrm{Ca}^{2+}$ chelator, nor $\mathrm{Ca}^{2+}$ indicator dye was present, but $0.2 \mathrm{mM} \mathrm{EGTA}$ as a slow $\mathrm{Ca}^{2+}$ buffer (Harafuji and Ogawa, 1980; Naraghi M, 1997), instead.

In the example shown (Fig. 19), pre-depolarizations of 4, 8, and $16 \mathrm{~ms}$ were followed by a long depolarization (50 ms; ISI = $20 \mathrm{~ms}$; red, green and blue traces, respectively). In addition, a single long depolarization (64 ms; black traces) was given. The time courses of cumulative release during the long depolarizations were quantified in the same way as described before for the other cross-depletion experiments (chapter 3.3.1).

As expected, pre-depolarization efficiently released vesicles from the FRP, and stimulation lengths of $8 \mathrm{~ms}$ (Fig. 19, green traces) and $16 \mathrm{~ms}$ (blue traces) released 2327 and 2476 FRP vesicles, respectively, being the same amount as for the $50 \mathrm{~ms}$ control pulse (2405 FRP vesicles; black traces). The $4 \mathrm{~ms}$ pre-depolarization (Fig. 19, red traces) was slightly too short to yield the same amount of fusion (2069 fast vesicles). The number of FRP vesicles left for the subsequent stimulus decreased with increasing length of pre-depolarization; however, again the sum of FRP vesicles released in total during the cross-depletion approach appeared to be higher compared to the single control pulse. While the two stimulus protocols with a first pulse of $4 \mathrm{~ms}, 8 \mathrm{~ms}$, or $16 \mathrm{~ms}$ length induced release of totally 3065 , 3367 , or 3068 FRP vesicles, respectively, the control pulse only triggered 2405 FRP vesicles for fusion. Moreover, the time constants of fast release during the second pulse correlated with the intensity of the first stimulus, becoming slower after longer pre-depolarization.

Please note, that for the example shown, peak EPSC sizes varied a lot, a phenomenon that is not typical for this kind of recordings (compare Fig. 11A). The voltage-clamp efficiency in the postsynaptic cell might have been not constant, and was possibly reduced during those recordings with smaller peak EPSC amplitudes.

As was already observed before (Fig. 16, 17), pre-stimulation led to a slowing of the FRP release time constant. In control, the long pulse induces release with a time constant of 2.7 ms (Fig. 19, black trace), but pre-depolarizations of 4, 8, and $16 \mathrm{~ms}$ length slowed release from the FRP, evoking time constants of $3.8,11.5$, and $15.0 \mathrm{~ms}$, respectively. 

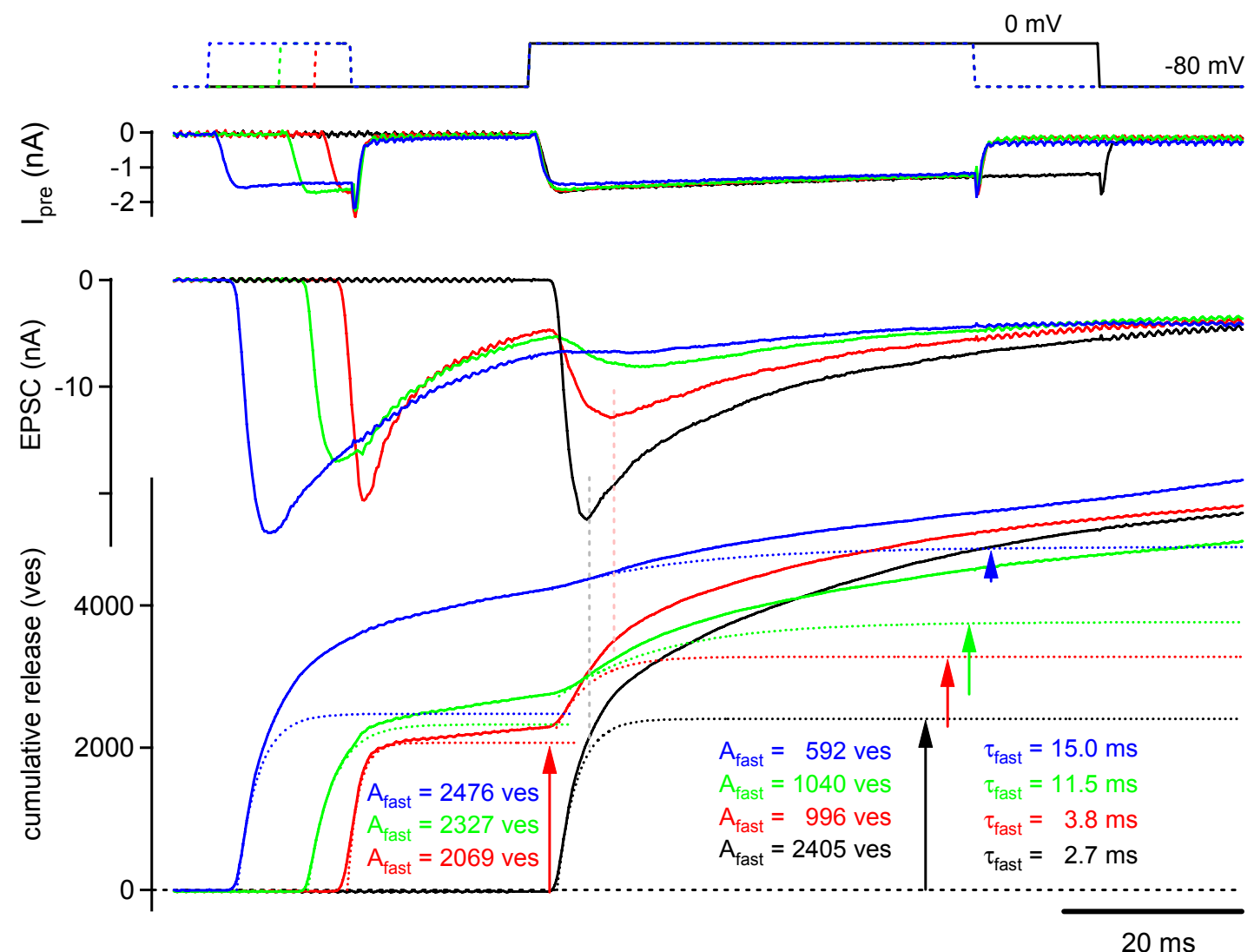

Fig. 19 Double-depolarization cross-depletion experiment to selectively pre-deplete the FRP

Long $50 \mathrm{~ms}$ depolarizations were preceded by short depolarizations of 4 , 8 , or 16 ms length (red, green and blue traces, respectively; $I S I=20 \mathrm{~ms}$ ), and pre-depletion effects on subsequent release have been compared to the unconditioned situation without precedent stimulation (black traces). The cumulative release traces have been analyzed for kinetics and amplitudes of release from the FRP (dotted traces; amplitude estimates indicated by arrows). 
Similar as done before (Fig. 18; chapter 3.3.1), estimates for amounts of release induced by long depolarizations after short pre-depolarizations were normalized to results from unconditioned control pulses within the same cell (Fig. 20A; $N=19$ ). In the cross-depletion approach, pre-depolarizations (4 - $16 \mathrm{~ms}$; ISI = $20 \mathrm{~ms}$ ) efficiently addressed the FRP (Fig. $20 \mathrm{~A} 1$ ), releasing on average $89 \pm 37 \%$ as compared to control, and the subsequent long pulse released another $26 \pm 28 \%$ of the FRP vesicles, thus the whole cross-depletion stimulation induced release of $113 \pm 33 \%$ of FRP vesicles, as compared to control. Thus, under $200 \mathrm{~nm}$ EGTA, the FRP was efficiently addressed by short pre-depolarization (26 \pm $28 \%$ ), and release from the FRP during the whole cross-depletion experiment was only marginally larger than during the long control pulse $(113 \pm 33 \% ; p=0.22)$. This slightly increased amount of release from the FRP during a cross-depletion experiment might result from fast refilling at high $\left[\mathrm{Ca}^{2+}\right]_{i}$ (Sakaba and Neher, 2001b). Comparing release efficiencies from the SRP showed that short pre-depolarizations and subsequent long pulses released 22 $\pm 23 \%$ and $55 \pm 28 \%$ SRP vesicles as compared to control, respectively (Fig. 20A2). This summed to a total amount of $77 \pm 21 \%$ SRP vesicles during the whole cross-depletion stimulation, being significantly less, as compared to control $(p=0.0003)$. Total release from the RRP, i.e. the sum of release from the FRP and the SRP, was similar during the crossdepletion stimulation as compared to control (Fig. 20A3; $94 \pm 18 \%, p=0.18$ ), while the pre-

Fig. 20 Cross-depletion to separate the FRP from the SRP during double-depolarization stimulation

A Effects of pre-stimulation (4 - $16 \mathrm{~ms}$ depolarizations) on release during subsequent depolarizations (50 ms) are illustrated. Amounts of cumulative release during the whole crossdepletion stimulation were normalized to the amount during a control stimulus (single $64 \mathrm{~ms}$ depolarization) within the same cell $(\mathrm{N}=19)$. During the cross-depletion sequence, the first pulse led to depletion of $89 \pm 37 \%, 22 \pm 23 \%$, and $44 \pm 21 \%$ of FRP, SRP, and total release, respectively (A1 $-\mathbf{A} 3,2^{\text {nd }}$ columns from left). The subsequent second pulse led to fusion of another $26 \pm 28 \%, 55 \pm 28$, and $48 \pm 26 \%$, respectively (A1 - A3, $3^{\text {rd }}$ columns), so that the whole cross-depletion sequence released $113 \pm 33 \%, 77 \pm 21 \%$, and $94 \pm 18 \%$ of the FRP, the SRP, and of total (A1 $-\mathbf{A} 3,4^{\text {th }}$ columns) as compared to control (A1 $-\mathbf{A} 3,1^{\text {st }}$ columns). B For the conditioned, second pulse, release time constants (B1) are displayed as function of pre-stimulation, and in the range of $2-16 \mathrm{~ms}$, biphasic release results were fitted with linear regressions in this double-logarithmic plot (dotted and dashed black lines). Slow release after 8 - $64 \mathrm{~ms}$ pre-depolarization was also fitted with a line (dashed red line). Without pre-stimulation, time constants were $3.6 \pm 2.1 \mathrm{~ms}$ and $66.4 \pm 22.3 \mathrm{~ms}$ with $1754 \pm 573$ and $3971 \pm 1906$ vesicles for the FRP and SRP on average, respectively (red symbols; $N=8$ ). C Amounts of release during whole cross-depletion sequences as functions of control depolarizations (64 ms) within the same cell are shwon. FRP release (closed triangles) and total release (crosses) are displayed for thee different cells (indicated by different colors). 

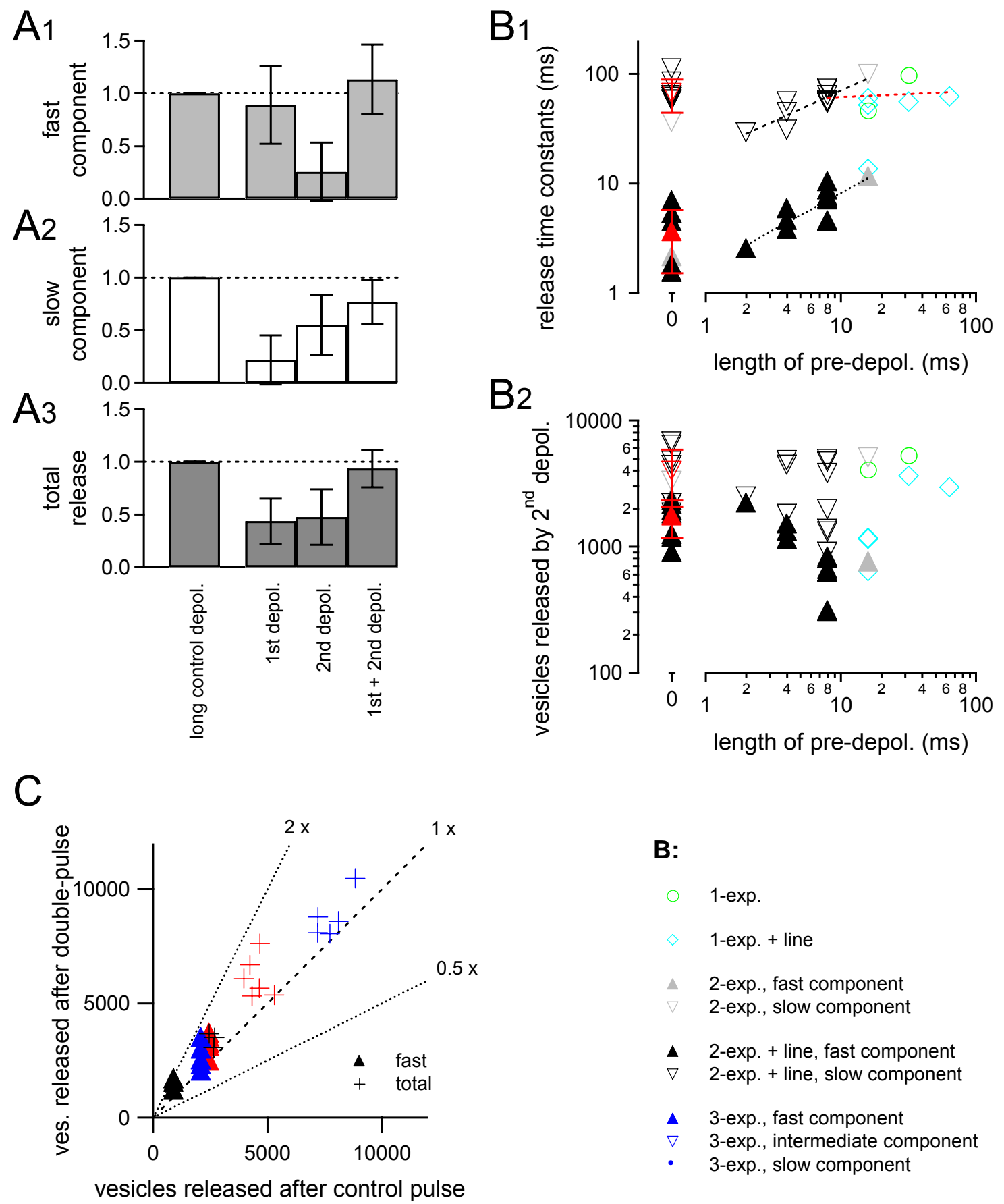

$\mathrm{B} 2$

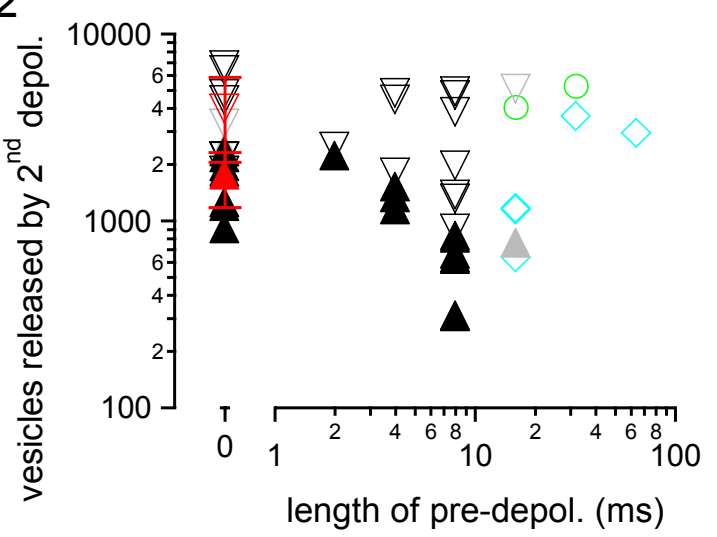

B:

1-exp

1-exp. + line

2-exp., fast component 2-exp., slow component

$\Delta \quad$ 2-exp. + line, fast component

$\nabla \quad$ 2-exp. + line, slow component

$\Delta \quad$ 3-exp., fast component

$\nabla \quad 3$ 3-exp., intermediate component

- 3-exp., slow component 
pulse and the subsequent long pulse each released about half of control (44 $\pm 21 \%$ and $48 \pm$ $26 \%$, respectively). So here, while total release from the RRP was the same for both, crossdepletion and control stimulation (Fig. 20A3, rightmost column), the tendency of more release from the FRP (Fig. 20A1, rightmost column) at the expense of release from the SRP (Fig. A2, rightmost column) due to double-pulses could be observed.

The already described slowing of the time constant of the fast release component after prestimulation (Figs. 16, 17, 18A, 19) could be observed in this set of experiments, as well. As shown in Fig. 20B1, without pre-stimulation, release occurred relatively fast with $3.6 \pm 2.1 \mathrm{~ms}$ (red closed triangles; $\mathrm{N}=8$ ), but with pre-pulses of $2 \mathrm{~ms}$ to $16 \mathrm{~ms}$ duration, the time constant of subsequent release changed from about $3 \mathrm{~ms}$ to $10 \mathrm{~ms}$ with a linear relationship on the double-logarithmic scale (Fig. 20B1, dotted line). Pre-depolarizations longer than $16 \mathrm{~ms}$ did not leave fast vesicles unreleased, as apparent from the '1-exponential' or '1-exponential + line' time courses observed during the second pulse (Fig. 20B1, see respective symbols). The slow component seemed to decelerate as well, with dependence on the conditioning pulse length (Fig. 20B1, dashed black line), but since its time constant of release was always bigger than $30 \mathrm{~ms}$, the range for fitting was relatively short (stimulus length of $50 \mathrm{~ms}$ ). This trend is rather ambiguous for convincing quantification of such a slow component. For predepolarizations longer than $4 \mathrm{~ms}$, the deceleration of SRP kinetics vanished (Fig. 20B1, dashed red line). In this range, time constants of slow release were similar to those in the unconditioned situation (66.4 $\pm 22.3 \mathrm{~ms}$; N= 8; Fig. 20B1, red open triangles).

Averaging time constants obtained in conditioned stimuli, subsequently applied after predepolarization of 2 to $16 \mathrm{~ms}$ length, yielded $6.6 \pm 2.7 \mathrm{~ms}$ for the FRP ( $\mathrm{N}=11$; not shown), being markedly slower than in unconditioned control with $3.6 \pm 2.1 \mathrm{~ms}(\mathrm{~N}=8$; Fig. 20B1, closed red triangles; $p=0.030$ ). The averaged release time constant for the SRP did not change after pre-depolarization ( $57.3 \pm 18.8 \mathrm{~ms} ; \mathrm{N}=11$; not shown), as compared to control $(66.4 \pm 22.3 \mathrm{~ms} ; \mathrm{N}=8$; Fig. 20B1, red open triangles; $p=0.24)$.

As mentioned before, longer conditioning depolarizations reduced the number of FRP vesicles remaining for the second pulse (Fig. 20B2; compare to Fig. 20A1). The maximal number of vesicles releasable was $1754 \pm 573$ and $3971 \pm 1906(\mathrm{~N}=8)$, for the FRP and SRP, respectively. The amount of FRP vesicles released during the second pulse decreased gradually due to increasing length of pre-depolarization, until no fast release was observed anymore. Inconsistently, the slow component was reduced only in some cases. Possibly, fast refilling of the SRP (Sakaba and Neher, 2001b; see also Methods, chapter 2.4.2) mixes with 
release from the SRP, and thus leads to overestimations of the real SRP size. In Fig. 20C, the amount of release during the whole cross-depletion stimulation is plotted against the corresponding amount of release during a single control stimulus within the same cell. In all three cells tested (Fig. 20C, different colors, respectively), amounts of fast release (closed triangles) and total release (crosses) were larger due to cross-depletion as compared to control. However, this might be explained by refilling of the fast release component, because this refilling component can be accelerated at high $\left[\mathrm{Ca}^{2+}\right]_{i}$ (Sakaba and Neher, 2001b), possibly becoming fast enough to account for about $20 \%$ refilling (Fig. 20C) within $100 \mathrm{~ms}$ (i.e. the time from onset of pre-depolarization to the end of the second pulse, as in these experiments) at transiently elevated, basal $\left[\mathrm{Ca}^{2+}\right]_{i}$ after the first stimulus.

\subsection{Submaximal release from the fast release component}

In $\mathrm{Ca}^{2+}$ uncaging experiments, during analysis of release properties depending on the stimulus strength in terms of $\left[\mathrm{Ca}^{2+}\right]_{i}$ (Fig. 14), an unexpected phenomenon occurred: the amount of release from the FRP at low $\left[\mathrm{Ca}^{2+}\right]_{\mathrm{i}}$ appeared to be smaller than at $\left[\mathrm{Ca}^{2+}\right]_{i}$ above about $10 \mu \mathrm{M}$ (Fig. 14E). Likewise, in cross-depletion experiments using paired depolarizations, a single pulse seemed to lead to incomplete depletion of the FRP (Fig. 20A1 and $\mathrm{C}$ ). At least in $\mathrm{Ca}^{2+}$ uncaging experiments, this submaximal amount of release was not due to stimulus cessation, because the increased $\left[\mathrm{Ca}^{2+}\right]_{i}$ stayed sufficiently high (chapters 2.3.2, 4.2). Moreover, submaximal release did not seem to be an artifact due to fit ranges being too small relative to the time constant of release, at least for release from the FRP. When two release components could be distinguished, FRP release time constants were always smaller than 20 ms (Fig. 14A), while the fit range of 100 ms exceeded a lower limit for reasonable estimates well enough. Thus, the phenomenon of incomplete FRP depletion might have had a physiological correlate, and it was analyzed and described in more detail. Moreover, the effect of submaximal release has already been observed previously at the squid giant synapse (Hsu et al., 1996), and in sea urchin eggs (Blank et al., 1998a).

As shown in Fig. 21, by continuous $\mathrm{Ca}^{2+}$ uncaging after flash-photolysis the $\left[\mathrm{Ca}^{2+}\right]_{\mathrm{i}}$ decay was aimed to be kept minimal, using the monochromator light and an increased concentration of the $\mathrm{Ca}^{2+}$ chelator for continuous $\mathrm{Ca}^{2+}$ uncaging after flash-photolysis (60\% monochromator light and $40 \%$ flash-light, $3 \mathrm{mM} \mathrm{DMN}$; see Methods, chapter 2.3.2). $\mathrm{Ca}^{2+}$ uncaging flash- 
stimuli of different intensities were given, to comprise a large range of $\left[\mathrm{Ca}^{2+}\right]_{i}$ and corresponding release rates. Subsequently after $\mathrm{Ca}^{2+}$ uncaging, a $50 \mathrm{~ms}$ depolarization was given to release the rest of the readily-releasable vesicles pool, with an inter-stimulus interval of $100 \mathrm{~ms}$, or on some cases $50 \mathrm{~ms}$ (Fig. 21 and Fig. 22, respectively).

As can be seen (Fig. 21), EPSC rise times accelerated with increasing $\mathrm{Ca}^{2+}$ uncaging efficiency, and peak EPSC amplitudes became larger. Analysis of the time courses of cumulative release in response to $\mathrm{Ca}^{2+}$ uncaging unveiled, that release kinetics became faster, and showed a biphasic release time course for $\left[\mathrm{Ca}^{2+}\right]_{i}$ above $5 \mu \mathrm{M}$, always being classified as '2-exponential + line'. The response to $4 \mu \mathrm{M}\left[\mathrm{Ca}^{2+}\right]_{i}$ (Fig. 21, green traces) showed a linear rise in its cumulative release, as was often observed at very low stimulus intensities (Fig. 14A). Here, a fast and a slow component could not be separated, probably because their release rates converged. In contrast, with stronger stimulation, separation of the FRP and SRP became more pronounced (Fig. 21, yellow traces), and finally very clear (red, blue and black traces). The regressions to the time courses of cumulative release yielded release time constant estimates of about $9 \mathrm{~ms}$ at $6 \mu \mathrm{M}\left[\mathrm{Ca}^{2+}\right]_{\mathrm{i}}$ (Fig. 21 , yellow traces) that steadily speeded up to less than a millisecond at $27 \mu \mathrm{M}$ (black traces). Furthermore, submaximal release from the FRP appeared. Stimulus intensities of $6 \mu \mathrm{M}, 7 \mu \mathrm{M}$, and $11 \mu \mathrm{M}$ $\left[\mathrm{Ca}^{2+}\right]_{\mathrm{i}}$ did not deplete the FRP completely, but released it only fractional with 663,1241 , and 1682 vesicles, respectively. This is unexpected from conventional models of synaptic release (Schneggenburger and Neher, 2000; Bollmann et al., 2000; chapter 4.1), since persistent stimulation, such as applied here, should finally lead to pool depletion, and different stimulus intensities would only affect release kinetics, not the amount of release. In the example shown (Fig. 21), $\left[\mathrm{Ca}^{2+}\right]_{i}$ stayed quite constant, and should not be the cause for cessation of fast release (chapters 2.3.2, 4.2). Also, the range for gaining parameters of release from regressions should have been sufficiently long, because 100 milliseconds are much more than needed for convincing approximations to kinetics faster than $10 \mathrm{~ms}$. Moreover, EPSC amplitudes and time courses indicate submaximal release already, in the sense that lower stimulus intensities led to smaller peak EPSC amplitudes, but the EPSCs did not cross over in their decay phases. Thus, EPSC charges differed, being much lower at weaker stimulation. These differences in EPSC charge even remained, when taking residual currents in the synaptic cleft due to delayed clearance of glutamate into account (Fig. 21, dashed traces; Methods, chapter 2.4.1). Only the strongest stimulation with $27 \mu \mathrm{M}\left[\mathrm{Ca}^{2+}\right]_{\mathrm{i}}$ depleted the FRP of 2312 vesicles completely, obvious from the lack of further release during the subsequent depolarization. In the other cases, weaker $\mathrm{Ca}^{2+}$ uncaging efficiencies led to correspondingly 

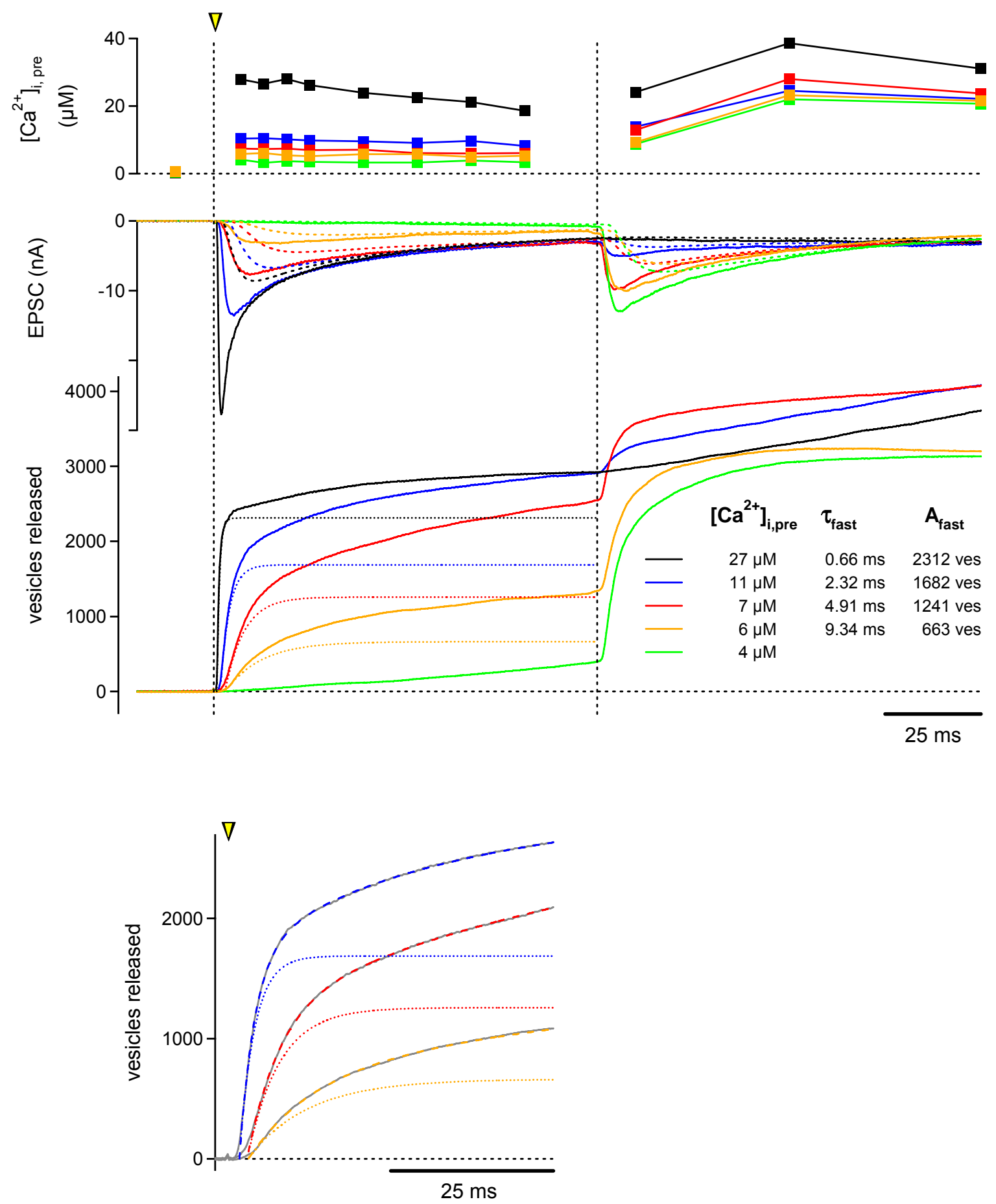

Fig. 21 Submaximal release from the FRP (example cell 1)

Five $\mathrm{Ca}^{2+}$ uncaging stimuli (yellow arrow) with different intensities have been applied within the same cell, and subsequently the cell was depolarized to $0 \mathrm{mM}$ for $50 \mathrm{~ms}(\mathrm{ISI}=100 \mathrm{~ms}$ ). Due to $\mathrm{Ca}^{2+}$ uncaging, $\left[\mathrm{Ca}^{2+}\right]_{i}$ increased to $4,6,7,11$, and $27 \mu \mathrm{M}$ (green, yellow, red, blue, and black traces, respectively). Deconvolved residual EPSCs (dashed traces) and time courses of cumulative release are shown. Time constants and amounts of FRP release are indicated (dotted lines; see also textbox). For clarity, responses to 6,7 , and $11 \mu \mathrm{M}\left[\mathrm{Ca}^{2+}\right]_{i}$ are shown again at expanded scales (inset). Note, that the peak EPSC for the strongest stimulus (black trace) was subject to amplifier saturation, and therefore was not included in the final dataset. 
more release from the FRP during the pool-depleting pulse, thereafter. Finally, both stimuli together released comparable amounts of FRP vesicles. Total release from the FRP by paired stimulation summed to $2313,1997,2153$, and 2346 vesicles at $27 \mu \mathrm{M}, 11 \mu \mathrm{M}, 7 \mu \mathrm{M}$, and $6 \mu \mathrm{M}\left[\mathrm{Ca}^{2+}\right]_{\mathrm{i}}$ after $\mathrm{Ca}^{2+}$ uncaging, respectively. Only in the case of $4 \mu \mathrm{M}\left[\mathrm{Ca}^{2+}\right]_{\mathrm{i}}$ both stimuli led to fusion of only 1349 FRP vesicles in total.

The same tendency of submaximal release was observed in the other example shown (Fig. 22). Here, the strongest $\mathrm{Ca}^{2+}$ uncaging stimulus to $18 \mu \mathrm{M}\left[\mathrm{Ca}^{2+}\right]_{i}$ (Fig. 22, black traces) was also potent enough to deplete the FRP completely, as can be seen by the ineffective subsequent depolarization. In this example, release in response to a $\mathrm{Ca}^{2+}$ uncaging stimulus to $3 \mu \mathrm{M}\left[\mathrm{Ca}^{2+}\right]_{\mathrm{i}}$ was classified as '1-exponential + line' (Fig. 22, red trace), whereas in the other example cell, a linear increase in cumulative release was observed at $4 \mu \mathrm{M}\left[\mathrm{Ca}^{2+}\right]_{i}(\mathrm{Fig}$. 21, green traces). Please note, that in contrast to the other example shown (Fig. 21), here $\left[\mathrm{Ca}^{2+}\right]_{\mathrm{i}}$ decayed slightly after flash-photolysis (Fig. 22). However, the overall conclusion regarding submaximal release was the same, only high stimulus intensities could overcome a cessation of fast release. $\mathrm{Ca}^{2+}$ uncaging to $3 \mu \mathrm{M}, 7 \mu \mathrm{M}$ and $18 \mu \mathrm{M}\left[\mathrm{Ca}^{2+}\right]_{\mathrm{i}}$ induced release of 266,801 , or $1720 \mathrm{FRP}$ vesicles, and together with the subsequent depolarizations of totally 1179,1122 , or 1720 FRP vesicles, respectively.

The dose-response relationship of cumulative release as a function of $\left[\mathrm{Ca}^{2+}\right]_{\mathrm{i}}$ has been summarized for several cells tested (Fig. 23). To take cell-to-cell variability in pool sizes into account, amounts of release at $\left[\mathrm{Ca}^{2+}\right]_{i}$ different from $10 \mu \mathrm{M}$ to $15 \mu \mathrm{M}$ were normalized to those at $10 \mu \mathrm{M}$ to $15 \mu \mathrm{M}$ (Fig. 23A). Each cell was marked with a different color code, and normalized cumulative release estimates from a single cell were connected by lines. The tendency of submaximal release became clearly visible for the FRP (Fig. 23A1), and even for stimulation strengths above $20 \mu \mathrm{M}\left[\mathrm{Ca}^{2+}\right]_{\mathrm{i}}$, amounts of release increased further on for the two cells obtained (red and orange data). On the contrary, SRP estimates showed the opposite behavior and progressively became smaller (Fig. 23A2). But here, scatter was high, and one of only two cells stimulated beyond $20 \mu \mathrm{M}\left[\mathrm{Ca}^{2+}\right]_{i}$ exhibited further reduction in release amounts (red data points), while in the other case the amount of release stayed rather constant (orange data points). Averaging data within three bins, one at $3 \mu \mathrm{M}$ to $10 \mu \mathrm{M}$ $\left[\mathrm{Ca}^{2+}\right]_{\mathrm{i}}$, one at $10 \mu \mathrm{M}$ to $15 \mu \mathrm{M}$, and one at $20 \mu \mathrm{M}$ to $45 \mu \mathrm{M}$ (Fig. 23B), yielded an estimate for the amount of release from the FRP of $65 \pm 30 \%$ at low $\left[\mathrm{Ca}^{2+}\right]_{i}$ (Fig. 23B1, left column), being significantly different from estimates at the mid $\left[\mathrm{Ca}^{2+}\right]_{i}$ range $(p=0.00048 ; N=15)$. At high $\left[\mathrm{Ca}^{2+}\right]_{i}$ (Fig. 23B1, right column), $143 \pm 20 \%$ could be released, but significance was low due to the few data points obtained $(p=0.062 ; N=3)$. The estimates of amounts of 


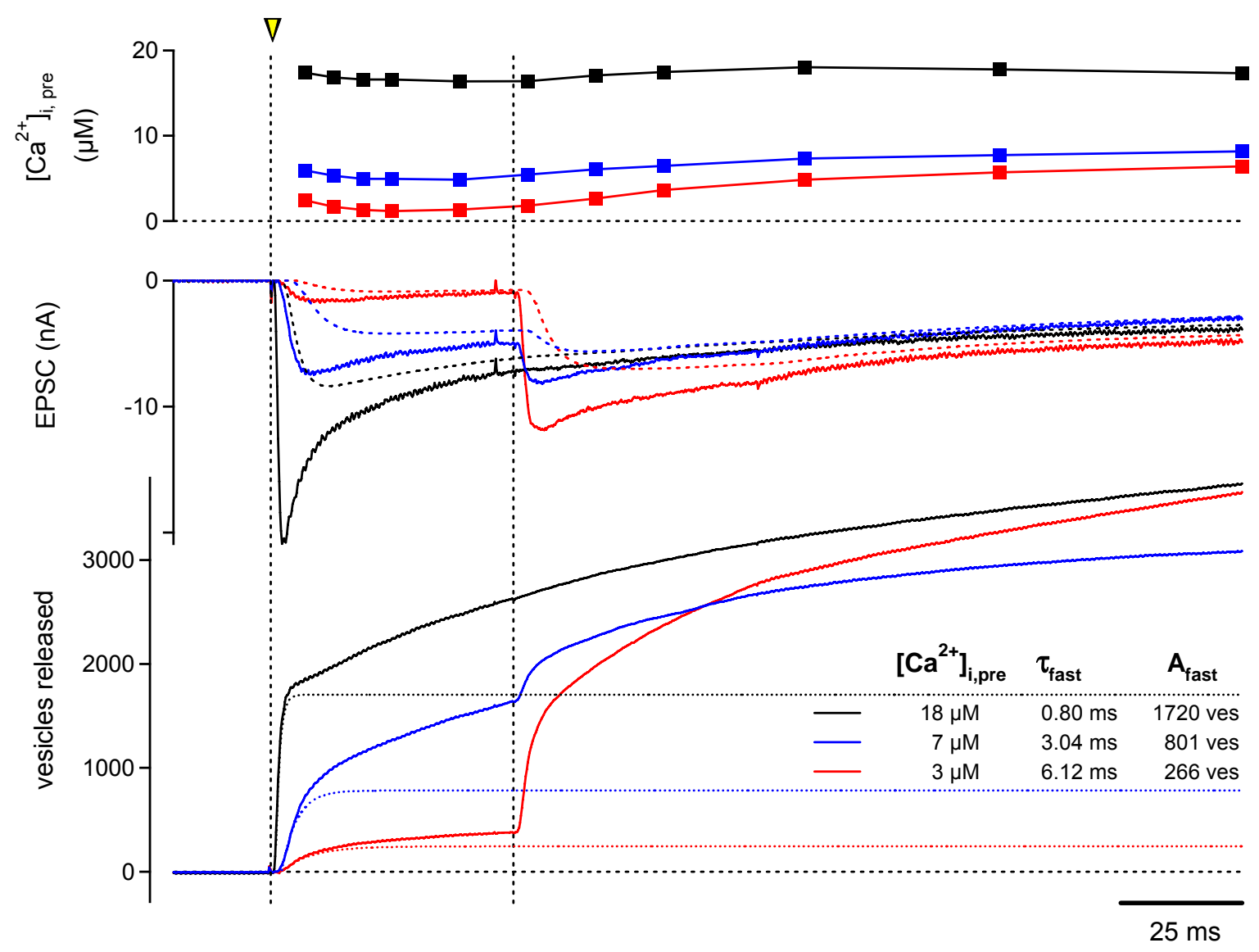

Fig. 22 Submaximal release from the FRP (example cell 2)

The same experimental approach as in Fig. 21 was performed in a different cell. $\left[\mathrm{Ca}^{2+}\right]_{\mathrm{i}}$ increased to 3,7 , and $18 \mu \mathrm{M}$ (red, blue, and black traces, respectively). Subsequent to $\mathrm{Ca}^{2+}$ uncaging, the cell was depolarized to $0 \mathrm{mV}$ for $50 \mathrm{~ms}$ (ISI = $50 \mathrm{~ms}$ ). 
cumulative release from the SRP decreased significantly from $152 \pm 66 \%$ at low stimulus intensities (Fig. 23B2, left column; $p=0.0089$ ) to a level of $68 \pm 39 \%$ at $\left[\mathrm{Ca}^{2+}\right]_{i}$ above $20 \mu \mathrm{M}$ (right column; $p=0.29$ ). Thus, the FRP and the SRP reacted in an opposite way: it seemed as if the amount of releasable vesicles from the FRP increased at the expense of the SRP.

Fig. 23C confirms that release efficiency from the FRP by $\mathrm{Ca}^{2+}$ uncaging strongly depended on the stimulus intensity. The amount of cumulative release after a $\mathrm{Ca}^{2+}$ uncaging stimulus was normalized to the summed amount of release after the same $\mathrm{Ca}^{2+}$ uncaging stimulus together with the subsequent depolarization. These pool size independent estimates for release (Fig. 23C) showed a strong dependence of amounts of fast release on $\left[\mathrm{Ca}^{2+}\right]_{\mathrm{i}}(\mathrm{Fig}$. 23C, closed triangles), elucidated by a linear regression to the semi-logarithmic data in the range of $5 \mu \mathrm{M}$ to $15 \mu \mathrm{M}\left[\mathrm{Ca}^{2+}\right]_{\mathrm{i}}$ (dotted red line). In the same range, a linear regression to the normalized SRP estimates (Fig. 23C, open triangles) did not show a marked slope (dashed red line), however it stayed rather constant at about two thirds of the total SRP size. But since the scatter was quite high, a significance of the independence of the amount of SRP release from $\left[\mathrm{Ca}^{2+}\right]_{i}$ was doubtful. The fractions of cumulative release induced by $\mathrm{Ca}^{2+}$ uncaging (Fig. 23C) were averaged in two groups of $\left[\mathrm{Ca}^{2+}\right]_{i}$. Similar as done before (Fig. 23B), a low $\left[\mathrm{Ca}^{2+}\right]_{i}$ range from $3 \mu \mathrm{M}$ to $10 \mu \mathrm{M}$ (Fig. 23D, three leftmost columns), and a mid $\left[\mathrm{Ca}^{2+}\right]_{\mathrm{i}}$ range of $10 \mu \mathrm{M}$ to $15 \mu \mathrm{M}$ (three rightmost columns) was defined for yielding averages within these bins. In the low $\left[\mathrm{Ca}^{2+}\right]_{\mathrm{i}}$ range, a Ca ${ }^{2+}$ uncaging stimulus released about $49 \pm 22 \%$ of

Fig. 23 Submaximal release from the FRP

A Amounts of release from the FRP (A1) and SRP (A2) are plotted as a function of $\left[\mathrm{Ca}^{2+}\right]_{\text {i. }}$. Results were normalized to estimates in a range of $10-15 \mu \mathrm{M}\left[\mathrm{Ca}^{2+}\right]_{\mathrm{i}}$, as obtained within the same cell (data from a single cell is indicated by a specific color and symbols are connected in order of stimulation). B Binned results yielded averages of $65 \pm 30 \%$ and $143 \pm 20 \%$ at $3-10$ $\mu \mathrm{M}\left[\mathrm{Ca}^{2+}\right]_{\mathrm{i}}$ (left column), and $152 \pm 62 \%$ and $68 \pm 39 \%$ at 20 to $45 \mu \mathrm{M}$ (right column) for the FRP (B1) and SRP (B2), respectively. C Fractional release after $\mathrm{Ca}^{2+}$ uncaging as normalized to summed release after $\mathrm{Ca}^{2+}$ uncaging and subsequent depolarization $(50 \mathrm{~ms})$ is shown as a function of $\left[\mathrm{Ca}^{2+}\right]_{\mathrm{i}}$ after $\mathrm{Ca}^{2+}$ uncaging. Release from the FRP, SRP, and from total (RRP) is indicated (closed triangle, open triangles, and crosses, respectively). In the range of $5-15 \mu \mathrm{M}$ linear regressions to estimates from FRP and SRP were made in this semi-logarithmic plot (dotted and dashed red lines, respectively). D Results shown in $\mathbf{C}$ were binned for the low, and the mid $\left[\mathrm{Ca}^{2+}\right]_{i}$ range $(3-10 \mu \mathrm{M}$ and $10-15 \mu \mathrm{M}$, as in $\mathrm{B})$. Responses to $\mathrm{Ca}^{2+}$ uncaging had averaged fractions of $49 \pm 22 \%$ for the FRP, $59 \pm 30 \%$ for the SRP, and $44 \pm 24 \%$ for the RRP at low $\left[\mathrm{Ca}^{2+}\right]_{i}(\mathrm{~N}=49)$, and of $79 \pm 8 \%$ for the FRP, of $53 \pm 20 \%$ for the SRP, and of $66 \pm$ $9 \%$ for the RRP at mid $\left[\mathrm{Ca}^{2+}\right]_{i}(\mathrm{~N}=7)$, as compared to summed release after $\mathrm{Ca}^{2+}$ uncaging and subsequent depolarization. 
$\mathrm{A}_{1}$
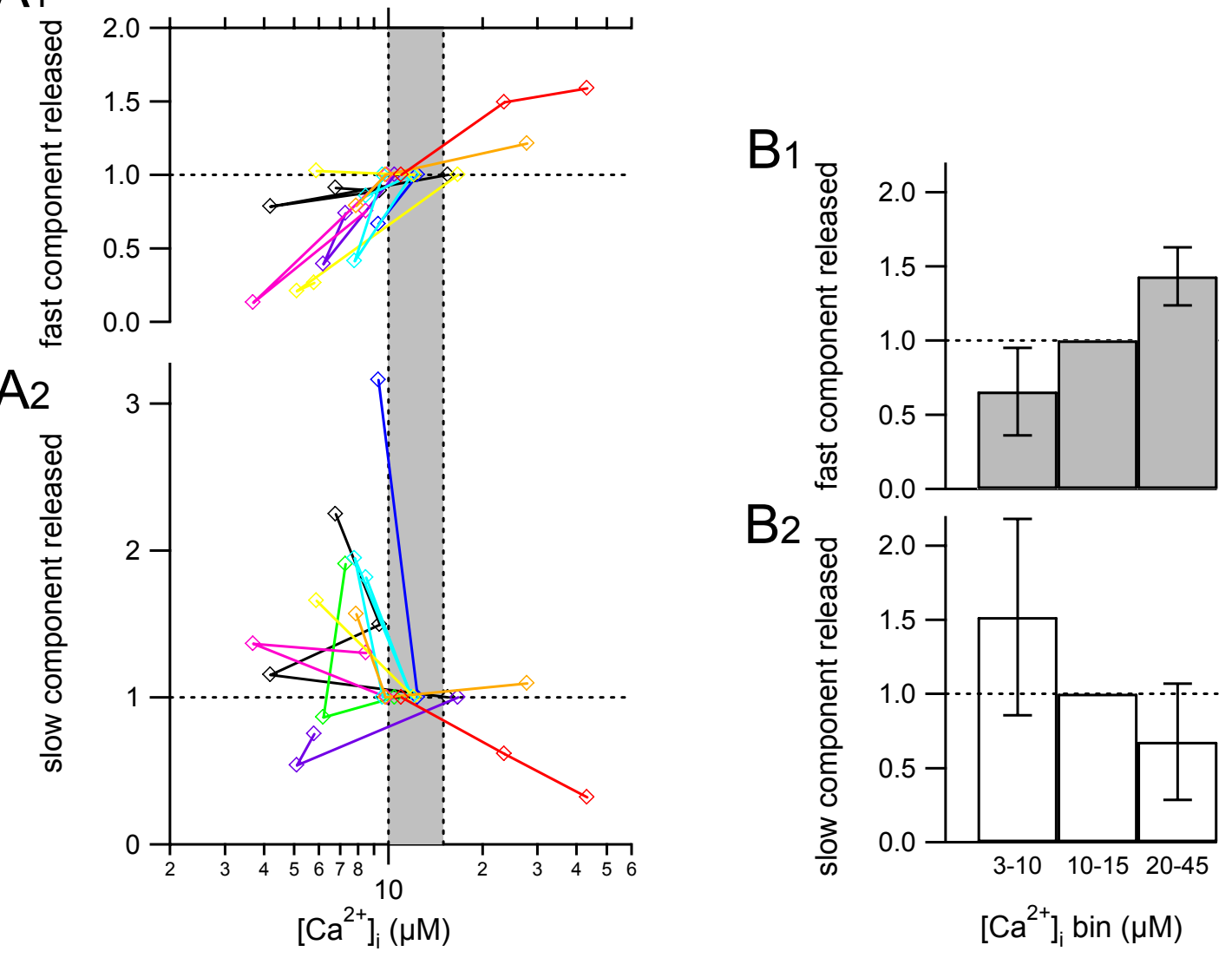

C

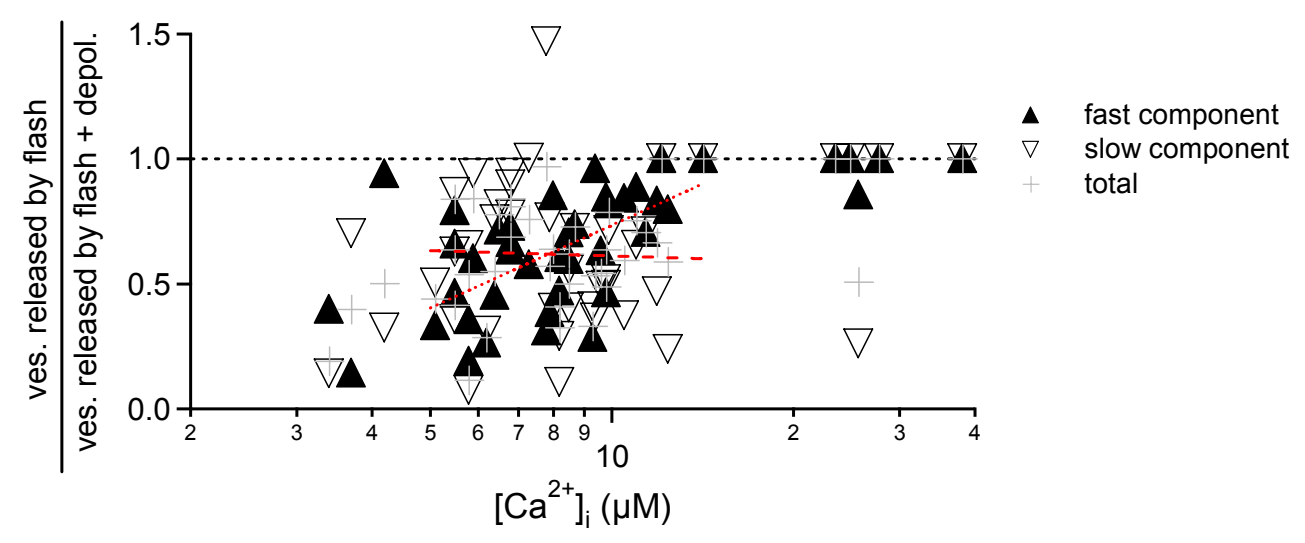

D

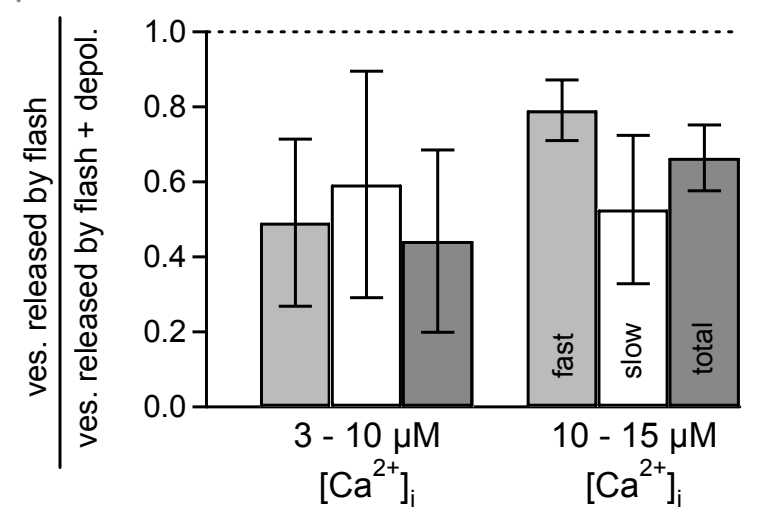


the FRP, as compared to summed release after both stimuli, $\mathrm{Ca}^{2+}$ uncaging and subsequent depolarization (Fig. 23D, $1^{\text {st }}$ column from left; $N=49 ; \mathrm{p}<0.0001$ ), and in the mid $\left[\mathrm{Ca}^{2+}\right]_{i}$ range, $79 \pm 8 \%$ of the FRP were released $\left(4^{\text {th }}\right.$ column from left; $\left.N=7 ; p=0.016\right)$. Thus, the $\mathrm{Ca}^{2+}$ uncaging stimulus was not able to deplete the FRP, as would be expected from the simplest depletion model at the calyx of Held synapse (Schneggenburger and Neher, 2000; Bollmann et al., 2000). Such a model would expect complete pool depletion for all $\left[\mathrm{Ca}^{2+}\right]_{\mathrm{i}}$, given that the stimulus persists.

For the SRP, the situation was similar. At $3 \mu \mathrm{M}$ to $10 \mu \mathrm{M}\left[\mathrm{Ca}^{2+}\right]_{\mathrm{i}}$, the $\mathrm{Ca}^{2+}$ uncaging stimulus release only $59 \pm 30 \%$ of the SRP (Fig. 23D, $2^{\text {nd }}$ column; $N=49 ; p<0.0001$ ), and at $10 \mu \mathrm{M}$ to $15 \mu \mathrm{M}\left[\mathrm{Ca}^{2+}\right]_{\mathrm{i}}, 53 \pm 20 \%$ of the SRP were released $(\mathrm{N}=7 ; \mathrm{p}=0.017)$.

When comparing the normalized amount of cumulative release from the FRP in response to $\mathrm{Ca}^{2+}$ uncaging (Fig. 23D) at $3 \mu \mathrm{M}$ to $10 \mu \mathrm{M}\left[\mathrm{Ca}^{2+}\right]_{i}$ with that at $10 \mu \mathrm{M}$ to $15 \mu \mathrm{M}$ (light grey columns), submaximal release became apparent, again. The fraction of $49 \pm 22 \%$ of the FRP released at relatively low $\left[\mathrm{Ca}^{2+}\right]_{\mathrm{i}}$ (Fig. $23 \mathrm{D}, 1^{\text {st }}$ column) was significantly smaller than the fraction of $79 \pm 8 \%$ of the FRP at mid $\left[\mathrm{Ca}^{2+}\right]_{i}\left(4^{\text {th }}\right.$ column; $\left.p<0.0001\right)$. Thus, increasing stimulus intensities progressively overcame the phenomenon of submaximal release from the FRP. A significant decrease in release efficiencies regarding the SRP could not be observed (Fig. 23D, comparing white columns; $p=0.74$ ). So an enlargement of the FRP size at expense of the SRP size was not undisputed, but the tendency could be observed (Fig. 23B). 


\section{Theoretical description of experimental results}

Models for the kinetics of release from the FRP at the calyx of Held synapse have already been described (Schneggenburger and Neher, 2000; Bollmann et al. 2000), using a $\mathrm{Ca}^{2+}$ sensor model in which successive binding of five $\mathrm{Ca}^{2+}$ ions leads to an irreversible final fusion step. $\mathrm{Ca}^{2+}$ binding was proposed to be either cooperative (Schneggenburger and Neher, 2000), or non-cooperative (Bollmann et al., 2000). Because the five-site $\mathrm{Ca}^{2+}$ binding model with intrinsic cooperativity also could describe the $\mathrm{Ca}^{2+}$ sensitivity of delays in release convincingly, being a critical parameter for the approximation of release kinetics, this model will be used as the basis for further biophysical considerations in this study (Fig. 24C).

The occurrence of two release components with distinct release kinetics could have been a result of different possible mechanisms, and several mechanisms for heterogeneous release were tested with descriptive models of $\left[\mathrm{Ca}^{2+}\right]_{i}$ dependent release. These models simulated cumulative release time courses according to their kinetic schemes, and according to the stimulus intensity in terms of the $\mathrm{Ca}^{2+}$ concentration. Then, the simulated time courses of cumulative release were analyzed in the same way as the experimental data (see Methods, chapter 2.4.2), for a convenient comparison.

First, the simplest model incorporated a kinetically homogeneous pool of vesicles, and assumed fast refilling, the latter probably appearing as a slow release component (Fig. 24A). Here, no distinction between an FRP and an SRP was made, only a homogeneous RRP and refilling of the RRP contributed to release.

Second, two kinetically distinct components of release were implemented as two parallel pools, being independent of each other, and both intrinsically differing in their $\mathrm{Ca}^{2+}$ sensitivities (Fig. 24B). Here, the RRP could be divided into an FRP and an SRP, but only refilling of the SRP was taken into account due to the extremely slow refilling of the fast release component (Sakaba and Neher, 2001b).

Third, a kinetically homogeneous pool of vesicles was assumed to be the source of both release components, fast and slow. After binding of $\mathrm{Ca}^{2+}$ to the $\mathrm{Ca}^{2+}$ sensor, fast vesicles could intrinsically convert into slow ones, decreasing the rate fusion for the converted vesicle. This intrinsic heterogeneity of release in a homogeneous pool of vesicles should account for the appearance of the fast and the slow release component. Furthermore, the ability of this model to predict the experimentally observed submaximal release was examined. 


\subsection{The single pool model}

Possibly, only a single, homogeneous pool of vesicles exists, and the slow release component observed in $\mathrm{Ca}^{2+}$ uncaging experiments (Fig. 12 and 14A) arose from refilling of this single component. In this case, release kinetics of slow release would not only be determined by the intrinsic $\mathrm{Ca}^{2+}$ sensitivity of the RRP, but rather by the relatively slow refilling rates, becoming the kinetic bottleneck.

At the calyx of Held synapse, the RRP refills in two phases (Sakaba and Neher, 2001b). During paired pre- and postsynaptic voltage clamp recordings, release induced by presynaptic depolarization occurred with a fast and a slow component (Sakaba and Neher, 2001 b; Figs. 10 and 19), the fast release component recovering slowly with a time constant of several seconds, the slow release component being refilled with a fast rate of maximally $10 \mathrm{ves} / \mathrm{ms}$. Assuming pool sizes of 1500 vesicles for either component (chapter 3.2; Fig. $14 \mathrm{E}$ ), this converts the fast rate of refilling into a time constant of about $100 \mathrm{~ms}$ (see Methods, chapter 2.4.2). Since in $\mathrm{Ca}^{2+}$ uncaging experiments the estimated time constants of release for the SRP approximated $100 \mathrm{~ms}$ at stimulus intensities below $10 \mu \mathrm{M}\left[\mathrm{Ca}^{2+}\right]_{\mathrm{i}}$ (Fig. 14A), the possibility of refilling being the origin of the slow release component was examined in more detail.

To simulate release from a kinetically homogeneous RRP (Fig. 25A), a cooperative five-site $\mathrm{Ca}^{2+}$ binding model with release dependent refilling (see scheme in Fig. 24C; Schneggenburger and Neher, 2000) was implemented using an Euler approach. Refilled vesicles were assumed initially to be in the $\mathrm{Ca}^{2+}$ free state, and the speed of refilling $r_{\text {ref }}$ was proportional to the fraction RRP depletion. Thus, a maximal rate of refilling $r_{\text {ref, max }}$ was

Fig. 24 Models of presynaptic release

Schemes of three different release models are shown: the single pool model $(\mathbf{A})$, the two pool model with intrinsic heterogeneity (B), and the single pool model with intrinsic heterogeneity (D). Kinetic differences in release are indicated by labeling the RRP with "FRP" and "SRP", and refilling is symbolized with " $R$ ". Active zones are displayed as thick membrane regions, and $\mathrm{Ca}^{2+}$ channels appear as two vertical cones. Black and brown arrows in $\mathbf{D}$ represent conversion into the fast releasing state $X$, or the slow releasing state $X^{\prime}$, respectively, and grey arrows stand for temporal progression. $\mathbf{C}$ Basic kinetic scheme for the $\mathrm{Ca}^{2+}$ sensor model triggering release in the models shown in $\mathbf{A}$ and $\mathbf{B}$. " $X_{\mathrm{NCa}}$ " represents the state of successive $\mathrm{Ca}^{2+}$ binding, with "N" as the number of $\mathrm{Ca}^{2+}$ ions bound, and "Res" stands for the reserve pool for refilling (see also Schneggenburger and Neher, 2000). E Kinetic scheme for the model illustrated in D. Symbols are used as in $\mathbf{C}$, and states marked with an apostrophe represent the 'desensitized' state (brown), while "F" stands for fusion. 

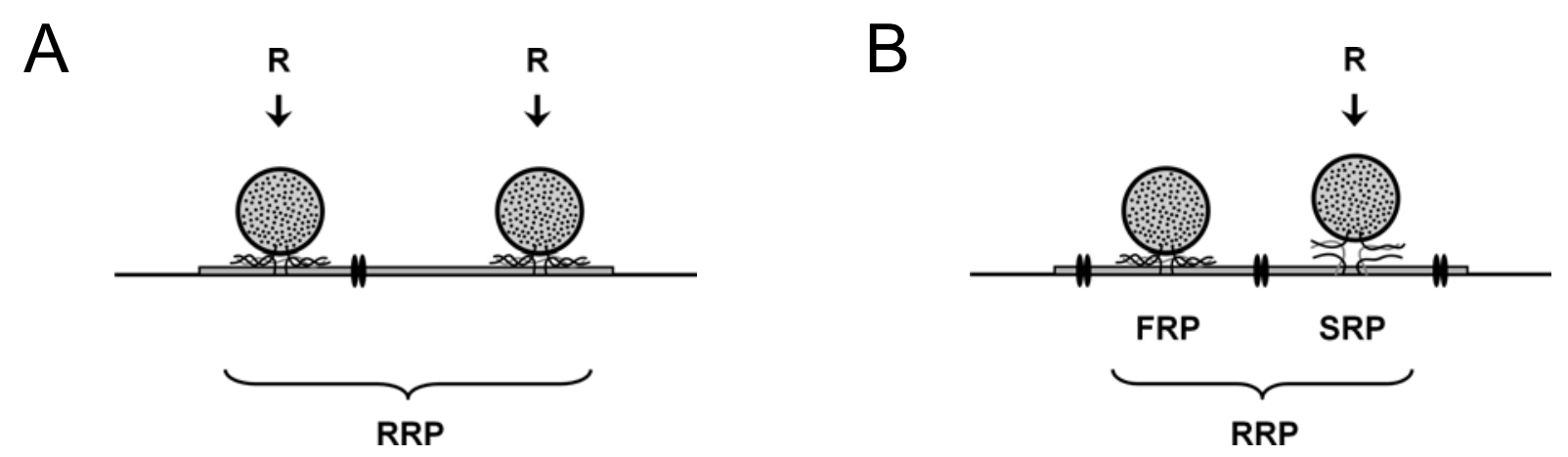

C

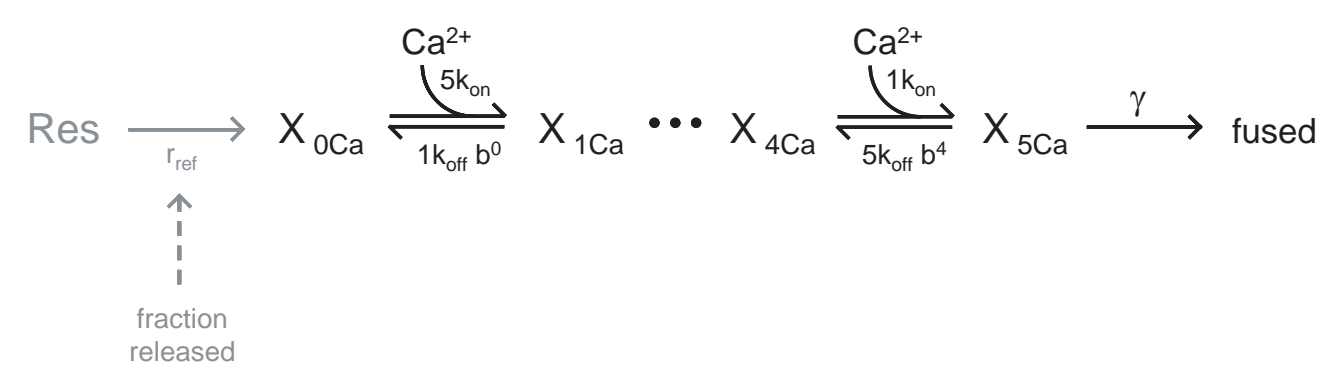

D
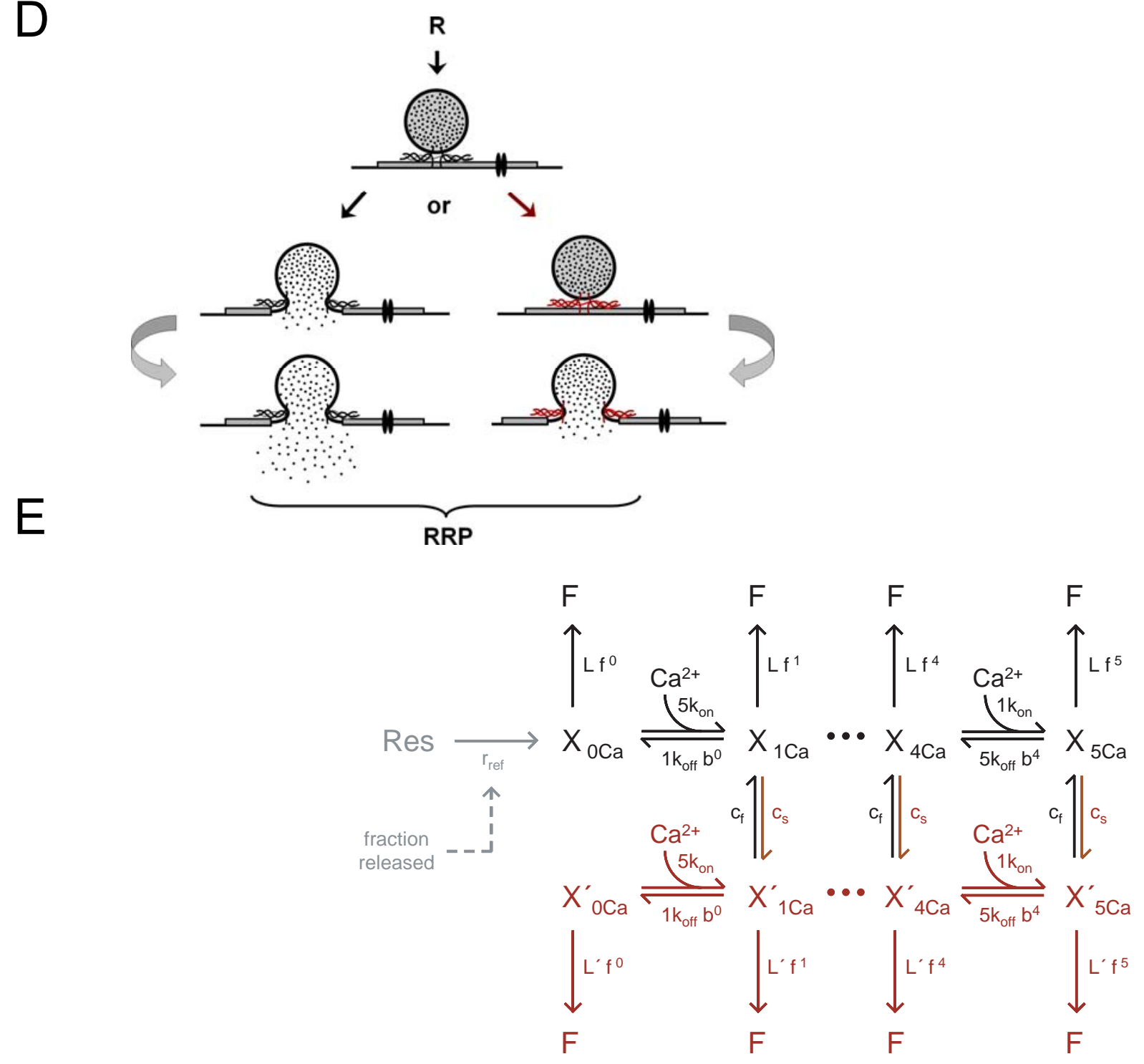
reached at a time point when no vesicle was left in the RRP, whereas for a completely replenished RRP, no refilling took place. The free model parameters included the on-rate $k_{\text {on }}$, the off-rate $k_{\text {off, }}$ and the cooperativity factor $b$ for the five $\mathrm{Ca}^{2+}$ binding steps, and furthermore, the final fusion rate $\gamma$, and the maximal refilling rate $r_{\text {ref, } \max }$ (Fig. 24C). These parameters were adjusted empirically to fit the experimentally obtained time constants of release, release rates, and times-to-peak release rate.

This is shown in Fig. 25. Time constants of release, peak release rates, amounts of vesicles released, and time-to-peak release rates obtained from simulations (Fig. 25C - F, colored symbols) were plotted on top of the experimental results (grey symbols, same as in Fig. 14) to adjust the model parameters for congruence with experimental results. Delay estimates of release were taken into account as well (not shown).

For illustration (Fig. 25B), simulated cumulative release time courses for different $\mathrm{Ca}^{2+}$ concentrations are shown (continuous black curves), together with the corresponding best regression obtained (dashed colored curves).

When taking refilling not into account, approximating the experimental results for the FRP yielded an on-rate $k_{\text {on }}$ of $1.20 \cdot 10^{8} \mathrm{M}^{-1} \mathrm{~s}^{-1}$, an off-rate $k_{\text {off }}$ of $8500 \mathrm{~s}^{-1}$, a cooperativity factor $b$ of 0.25 , and a final fusion rate $\gamma$ of $6000 \mathrm{~s}^{-1}$ (Fig. 25B1 and C - F, red symbols), comparing well to previous estimates (Schneggenburger and Neher, 2000; Felmy et al., 2003). Moreover, the RRP pool size was set to 2000 vesicles. Based on this set of parameters, an additional refilling into the $\mathrm{Ca}^{2+}$ unbound state $\mathrm{X}_{\text {OCa }}$ (Fig. 24C) with a rate of either 10 ves/ms (Fig. 25B2 and C - F, blue symbols) as a maximal experimental estimate (Sakaba and Neher, 2001b), or $100 \mathrm{ves} / \mathrm{ms}$ for a simulated extreme contribution of refilling (Fig. 25B2 and C - F, green symbols) was implemented.

As can be seen from the time courses of cumulative release, refilling with a rate of $10 \mathrm{ves} / \mathrm{ms}$ already led to marked contribution of release, obvious as a second, linearly rising release

Fig. 25 Simulated release: The single pool model

A A single pool model including refilling was implemented to simulate time courses of cumulative release in dependence of stimulation intensity (B, black traces for $\left[\mathrm{Ca}^{2+}\right]$ i of $1.5,2.4$, 3.7, 5.7, 8.8, 14, 21, 32, and $50 \mu \mathrm{M})$. Parameters were: $k_{\text {on }}=1.2 \cdot 10^{-8} \mathrm{M}^{-1} \mathrm{~s}^{-1}, k_{\text {off }}=8500 \mathrm{~s}^{-1}, \mathrm{a}$ $=1, \mathrm{~b}=0.25, \gamma=6000 \mathrm{~s}^{-1}$, pool size $=2000$ ves. Simulated cumulative release was fitted with regressions (B, dotted colored curves), and resulting release time constants (C), peak release rates $(D)$, amounts of release $(E)$, and times-to-peak release rate $(F)$ are plotted on top of experimentally obtained data (grey symbols). The model was implemented without refilling (B1; B - F red data), or with refilling rates of $10 \mathrm{ves} / \mathrm{ms}$ (B2; B - F blue data), or $100 \mathrm{ves} / \mathrm{ms}$ (B3; B $\mathbf{F}$ green data), and kinetic types of release are specified by different symbols for estimates derived from exponential fits (C, E, as indicated in the textbox). 
A
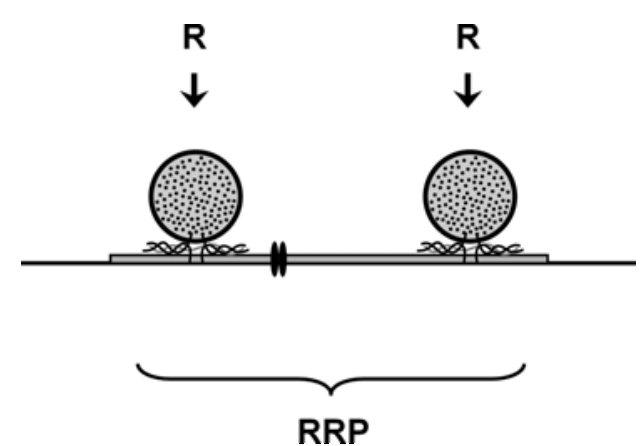

C - F, best fit types:

- 1-exp.

- 1-exp. + line

\ 2-exp., fast

$\nabla$ 2-exp., slow

- 2-exp. + line, fast

$\nabla$ 2-exp. + line, slow

\ 3-exp., fast

$\nabla$ 3-exp., mid

- 3-exp., slow

C

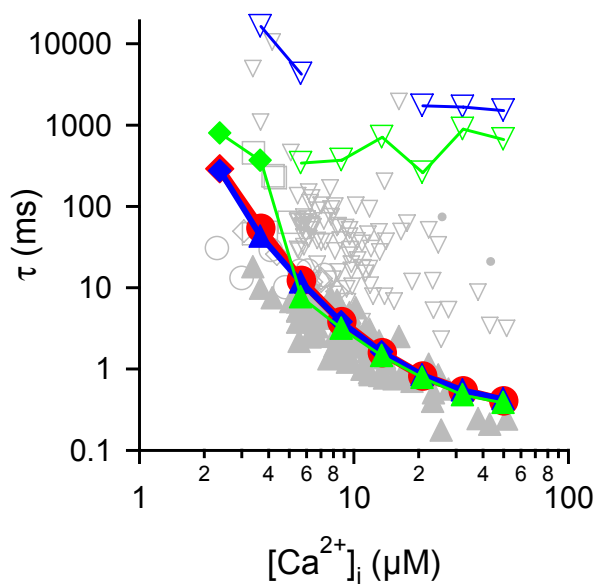

$E$

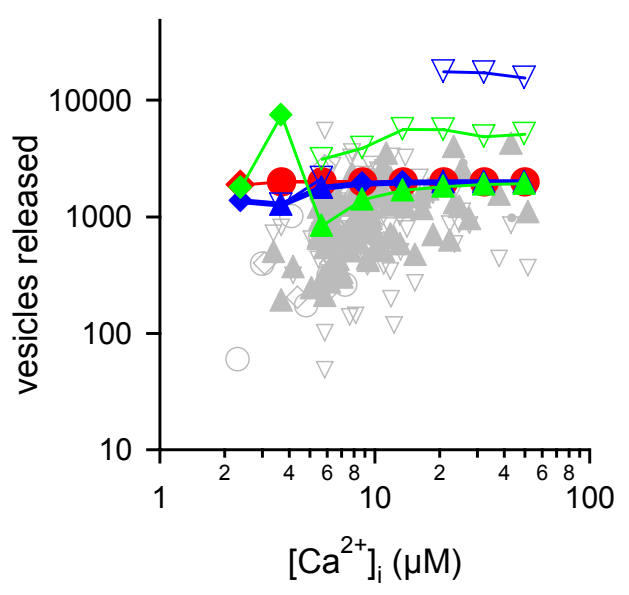

B1

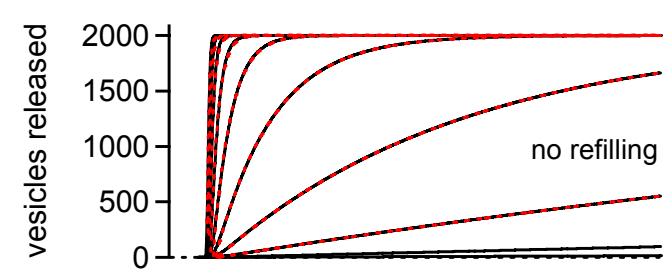

B2

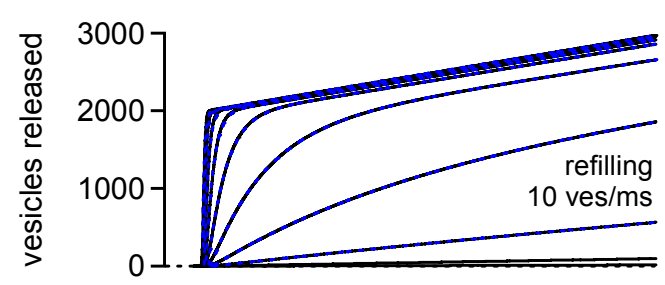

B3

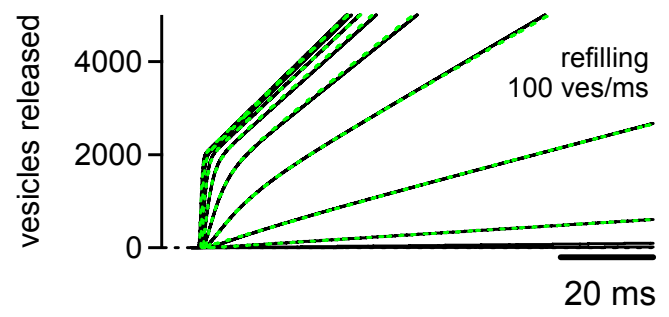

D

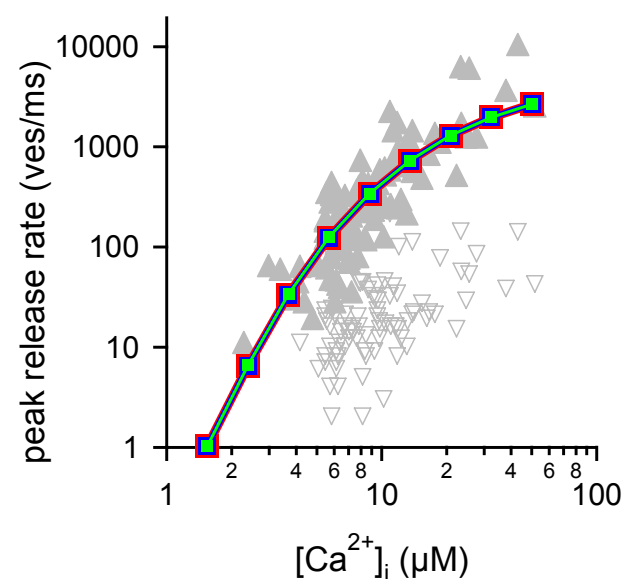

F

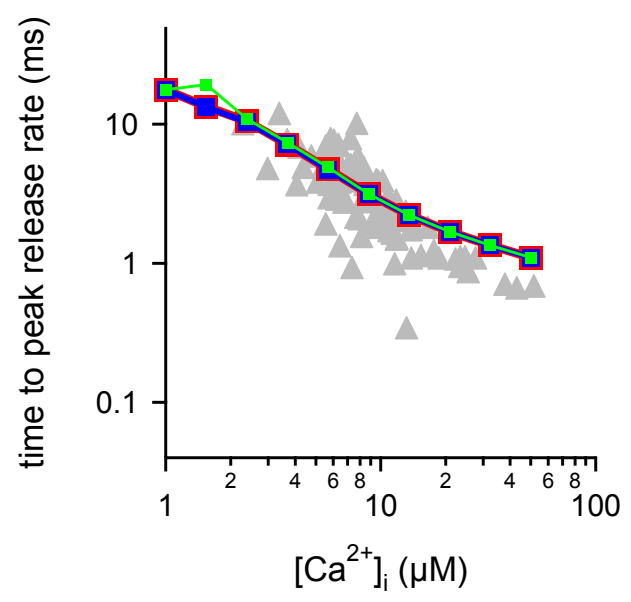


component (Fig. 25B2, compared to B1). Driving simulated refilling to a rather nonphysiological extreme of $100 \mathrm{ves} / \mathrm{ms}$ increased the contribution of this second, ongoing release component, being even bigger than that observed in experiments (compare with Figs. 12B - C, 16, 17). Thus, with refilling rates of 0 ves $/ \mathrm{ms}, 10 \mathrm{ves} / \mathrm{ms}$, or $100 \mathrm{ves} / \mathrm{ms}$, the range of possible contributions of refilling under physiological conditions should have been covered. The standard analysis approach (see Methods, chapter 2.4.2) of the simulated data always led to comparable estimates for release kinetics of the fast component (Fig. 25C - F, closed symbols). For peak release rates and times-to-peak release rate, results from the three conditions were indistinguishable, and at stimulus intensities above $5 \mu \mathrm{M}\left[\mathrm{Ca}^{2+}\right]_{\mathrm{i}}$, time constants of fast release and amounts of fast release compared well. Only with a refilling rate of $100 \mathrm{ves} / \mathrm{ms}$ and $\left[\mathrm{Ca}^{2+}\right]_{\mathrm{i}}$ steps to less than $5 \mu \mathrm{M}$, refilling could induce an apparent wrong estimate of fast release. Here, the relatively slow release kinetics approached that of the assumed fast refilling, so that both components became indistinguishable from each other by the analysis methods applied. As a consequence, below $5 \mu \mathrm{M}\left[\mathrm{Ca}^{2+}\right]_{\mathrm{i}}$ pool size estimates varied around the given size of 2000 ves (Fig. 25E, green symbols), and estimates for RRP release time constants were artificially increased, being intermingled estimates of the kinetics of release and refilling.

While a second release component was intrinsically and virtually absent in the implementation without refilling (Fig. 25B1), a refilling rate of $10 \mathrm{ves} / \mathrm{ms}$ resulted in a classification of cumulative release that was comprised of two exponentials at five out of eight $\mathrm{Ca}^{2+}$ concentrations (Fig. 25C - E, blue symbols). Refilling with a rate of $100 \mathrm{ves} / \mathrm{ms}$ led to the analytical finding that release included two release components in all cases above 4 $\mu \mathrm{M}\left[\mathrm{Ca}^{2+}\right]_{i}$ (Fig. 25C - E, green symbols). However, in none of these cases the resulting time constant of slow release matched the experimental results (Fig. 25C - E, open blue and green symbols), being five to a hundred times slower than the experimentally observed slow release component (Fig. 25C, open grey triangles). Furthermore, no dependence of the slow release time constants on $\left[\mathrm{Ca}^{2+}\right]_{i}$ could be observed, strongly contrasting the experimental results.

Thus, neither the experimentally approximated refilling rate of $10 \mathrm{ves} / \mathrm{ms}$ (Sakaba and Neher, $2001 \mathrm{~b}$ ), nor an even more potent rate of 100 ves/ms could mimic the appearance of the slow release component observed in $\mathrm{Ca}^{2+}$ uncaging experiments. This indicates that rapid refilling from a unlimited reserve pool ( $R$ in Fig. 25A) cannot result in the appearance of a slow release component (Figs. 10C, 11A, 12), when the synapse is stimulated with prolonged $\left[\mathrm{Ca}^{2+}\right]_{\mathrm{i}}$ elevations. 
A
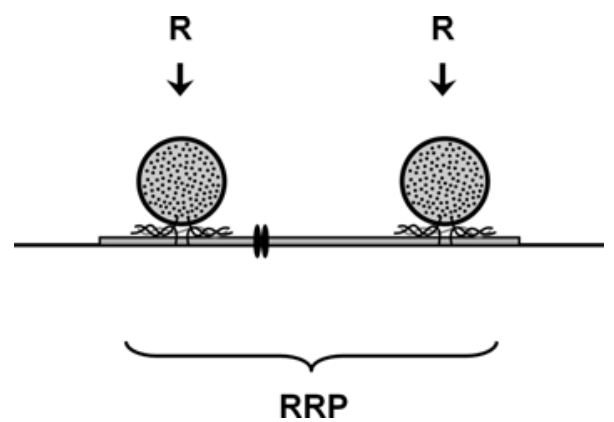

C

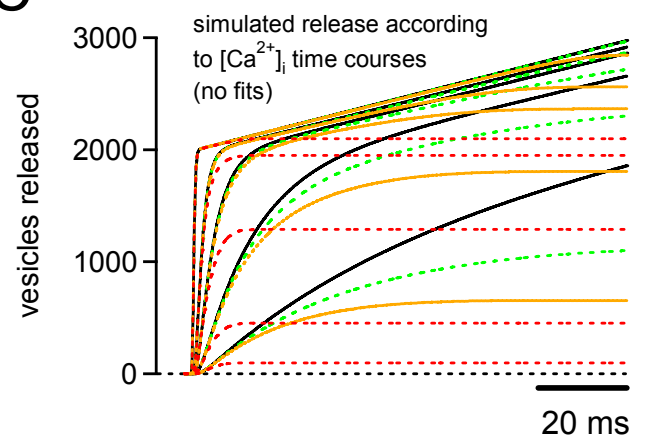

D

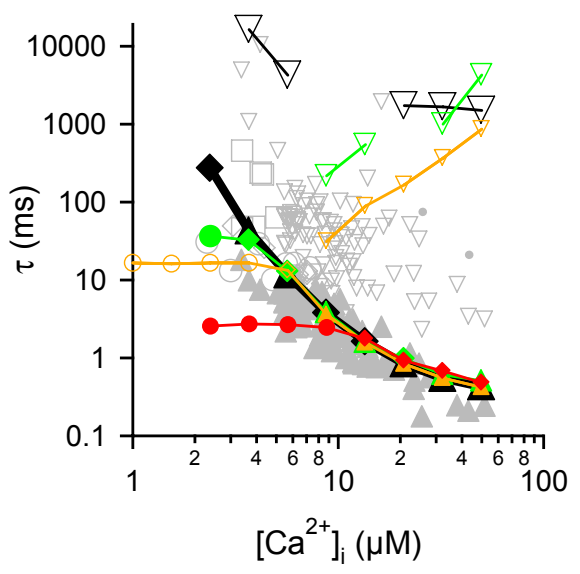

$\mathrm{F}$

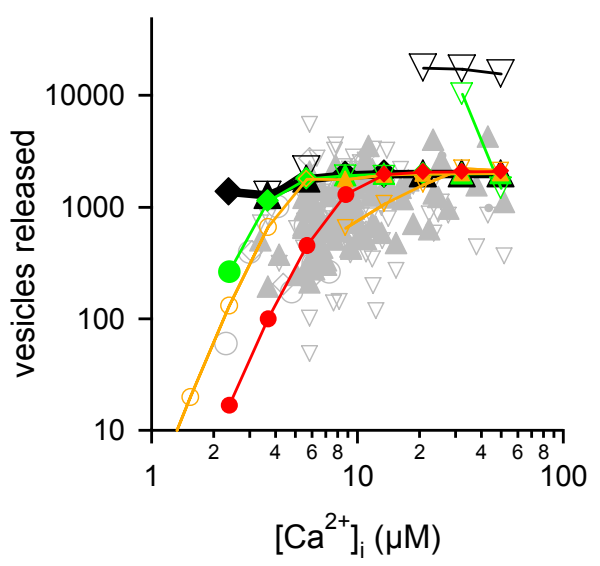

B

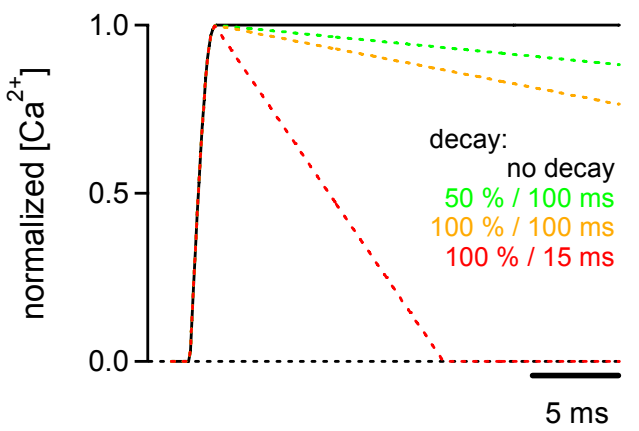

D - G, best fit types:

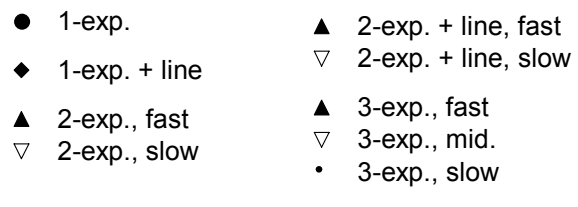

$E$

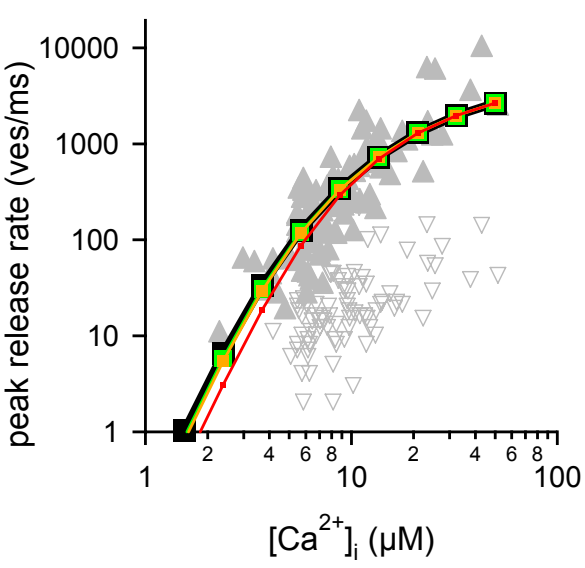

G

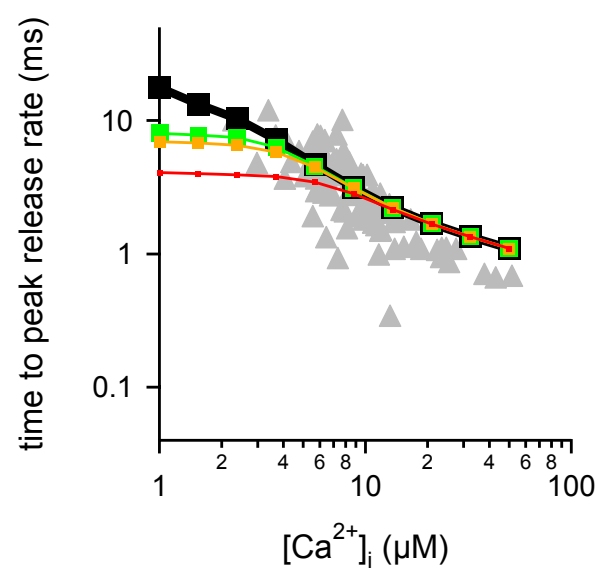

Fig. 26 Simulated release: The single pool model driven by decaying $\left[\mathrm{Ca}^{2+}\right]_{\mathrm{i}}$ Same as in Fig. 25, but the single pool model $(\mathbf{A})$ was driven by $\left[\mathrm{Ca}^{2+}\right]_{\mathrm{i}}$ curves that decayed with time after reaching its peak (B). Refilling was always set to $10 \mathrm{ves} / \mathrm{ms}$, and resulting cumulative release is shown without the regressions made (C, color code as in $\mathbf{B})$. 


\subsection{A fast $\left[\mathrm{Ca}^{2+}\right]_{i}$ decay cannot account for the appearance of the slow release component}

Clamping $\left[\mathrm{Ca}^{2+}\right]_{i}$ to a constant level after $\mathrm{Ca}^{2+}$ uncaging was aimed in the experiments performed, but could not be achieved perfectly (chapter 2.3.2; Figs. 16, 17, 21, 22). $\left[\mathrm{Ca}^{2+}\right]_{\mathrm{i}}$ rather decreased slightly, or even increased in some cases. A decrease in $\left[\mathrm{Ca}^{2+}\right]_{\mathrm{i}}$ might have led to a slowing of release, and this might have resulted in the appearance of an artificial second, slower release component.

To test for the effects of such a reduction in stimulus intensity with time, and to examine, if these effects could have resulted in the artificial appearance of a slow release component, the single pool model (chapter 4.1) was driven with different $\left[\mathrm{Ca}^{2+}\right]_{i}$ time courses. The typical time course, as used before (chapter 4.1), increased sigmoidally to its peak within $1.5 \mathrm{~ms}$ and then stayed constant (Fig. 26B, black trace). This was compared to three other simulations, in which the stimulus intensity decreased from its peak value at $1.5 \mathrm{~ms}$ by $50 \%$ within $100 \mathrm{~ms}$ (Fig. 26B, green trace), by $100 \%$ within $100 \mathrm{~ms}$ (yellow trace), or by $100 \%$ within $15 \mathrm{~ms}$ (red trace).

The rate of refilling of the intrinsically homogeneous RRP was set to $10 \mathrm{ves} / \mathrm{ms}$ in all cases, and the resulting time courses of cumulative release (some examples shown in Fig. 26C) was analyzed as stated before (chapters $2.4 .2,4.1$ ). As can be seen in comparison to control (Fig. 26C, black traces), release slowed down with time when driven by a decaying $\left[\mathrm{Ca}^{2+}\right]_{\mathrm{i}}$ signal (colored traces). The faster the $\left[\mathrm{Ca}^{2+}\right]_{i}$ decay was, the earlier release stopped.

Comparing the extracted kinetic parameters of simulated release to the experimentally observed results (Fig. 26D - G), no condition of simulation could account for the slow release component observed (open symbols). On the contrary, an artificially appearing slow release component showed an inverse $\left[\mathrm{Ca}^{2+}\right]_{i}$ dependence as compared to experimental results (Fig. $26 \mathrm{D}$, open orange and green triangles). Interestingly, a fast $\left[\mathrm{Ca}^{2+}\right]_{i}$ decay could emulate the tendency of submaximal release observed at low stimulus intensities (Fig. 26F, red symbols). But the marked deviation of the corresponding release time constants and times to peak release rate (Fig. 26D and G, closed red symbols) towards faster kinetics as compared to control (closed grey symbols) stood in clear opposition to the experimentally observed mechanism of submaximal release. Thus, submaximal release could be induced by cessation of stimulation, as expected, but at the same time, other kinetic parameters changed as well, and therefore disagreed with experimental findings.

Moreover, fast attenuation in stimulus intensities had a slight effect on release properties at very low stimulus intensities below $5 \mu \mathrm{M}\left[\mathrm{Ca}^{2+}\right]_{\mathrm{i}}$, but could not account for the appearance of two kinetically distinct release components as observed experimentally. 


\subsection{A two pool model with intrinsic heterogeneity}

Since the single pool model including refilling could not predict the appearance of the two kinetically distinct release components as observed in $\mathrm{Ca}^{2+}$ uncaging experiments, a model simulating intrinsic heterogeneity in $\mathrm{Ca}^{2+}$ sensitivities was tested (Fig. 27A). This model assumed independence between the FRP and SRP, both being based on the same five-site $\mathrm{Ca}^{2+}$ binding mechanism (Fig. 24C). Refilling of the SRP was taken into account only for the $\mathrm{SRP}$, because of the negligibly slow refilling rate of the fast release component (Sakaba and Neher, 2001b; chapter 2.4.2). The free parameters of the fast component were the same as before (chapter 4.1), and those of the slow component were adjusted to reproduce the experimental results for the SRP, as was done in a similar way when a slow release component in mouse chromaffin cells was described (Voets T, 2000). In that study, using a three-site $\mathrm{Ca}^{2+}$ binding model, the slow release component was well simulated with an about tenfold reduced on-rate $k_{\text {on }}$, off-rate $k_{\text {off }}$, and final fusion rate $\gamma$, as compared to the fast release component. Here, at the rat calyx of Held synapse, only $k_{\text {on }}$ and $k_{\text {off }}$ were reduced, but for these two parameters also a tenfold reduction was found to describe the experimental data for the SRP reasonably well (Fig. 27C - E, open red and blue symbols).

The time courses of cumulative release were simulated assuming either no refilling (Fig. 27B1), or a refilling rate of $10 \mathrm{ves} / \mathrm{ms}$ (Fig. 27B2). For either case, the biphasic behavior became apparent at stimulus intensities above $10 \mu \mathrm{M}\left[\mathrm{Ca}^{2+}\right]_{i}$, as for the fastest four cumulative release traces shown (Fig. 27B1, B2). Here, the differences in release kinetics produced a hump in the time course of cumulative release at a point where the FRP was almost depleted, but release from the SRP had just started. This effect was more obvious looking at the release rate traces (Fig. 27B3, corresponding to traces in 21B1 that were marked with asterisks in the according color), because at $\left[\mathrm{Ca}^{2+}\right]_{\mathrm{i}}$ above $10 \mu \mathrm{M}$, the release rates peaked in two temporally separated maxima (Fig. 27B3, black and yellow traces), representing the FRP and SRP, respectively. Importantly, neither two peaks in release rates, nor the described hump in the time course of cumulative release were observed in the electrophysiological recordings obtained for this study (Figs. 12, 16, 17, 21, 22; but compare also Fig. 22, black trace). But maybe this was due to filter effects of the EPSC deconvolution applied (chapter 2.4.1), because the deconvolution parameters resulted from empirical adjustments by eye. Even though being not likely, small humps in the cumulative release time course, such as those in the simulations (Fig. 27B), could have been leveled out in this way. However, the simulated release time courses could still be approximated with the standard fitting routine, and the results from classified regressions described the experimentally obtained parameters of the FRP and SRP well (Fig. 27C - F). Including a 
refilling with a rate of $10 \mathrm{ves} / \mathrm{ms}$ (blue symbols) did not lead to different results for the FRP, or the SRP, as compared to simulations without any refilling (red symbols).

Note, that differences between regressions and the corresponding cumulative release traces (Fig. 23B1, B2, dashed traces compared to continuous traces) most likely occurred due to the missing delay of the slow release component in the regression algorithms applied (see Methods, chapter 2.4.2). In these fitting routines, all exponential components started with the same delay as the fastest component, which obviously was not true for the simulated traces (Fig. 27B).

Not only estimates for release parameters of the FRP agreed well with experimental results (Fig. 27C $-\mathrm{F}$ ), but also the emerging slow release component described the $\left[\mathrm{Ca}^{2+}\right]_{\mathrm{i}}$ dependence of release time constants, and peak release rates well (Fig. 27C, open symbols, and 27D, respectively). For the estimated amount of release (Fig. 27E), a slight effect similar to submaximal release appeared. This was unexpected, because both simulated components were mutually independent, such that they both should have led to constant pool size estimates, irrespective of the stimulation strength. Thus, the pool size misinterpretation here was a consequence of the analysis applied. However, this effect clearly differed from the experimentally observed submaximal release (compare Fig. 27E, grey symbols, and Fig. 23A). The amount of fast release rather decreased with increasing $\left[\mathrm{Ca}^{2+}\right]_{\mathrm{i}}$, while the amount of slow release increased, which was clearly opposite to experimental findings.

In summary, this model was able to predict the experimentally derived kinetics of release, independent of fast refilling of the less $\mathrm{Ca}^{2+}$ sensitive component (SRP; Fig. 27C - F, compare red and blue symbols). But the simulated time courses of release showed a much clearer distinction between fast and slow release as compared to results obtained in experiments, and furthermore, the effect of submaximal release (chapter 3.4 ) could not be reproduced by this model.

Fig. 27 Simulated release: The two pool model with intrinsic heterogeneity

Same as in Fig. 25, but release was simulated using a model of two independent, parallel pools of vesicles with intrinsically different $\mathrm{Ca}^{2+}$ sensitivities (FRP and SRP; A). Model parameters were the same as Fig. 25, but $k_{\text {on }}$ and $k_{\text {off }}$ for the SRP were 10-times slower as compared to the FRP. Refilling was either omitted (B1), or accounted for the SRP only with a rate of $10 \mathrm{ves} / \mathrm{ms}$ (B2). B3 Release rates for three different stimulus intensities are shown (corresponding traces of cumulative release are marked with asterisks in B1). 
A

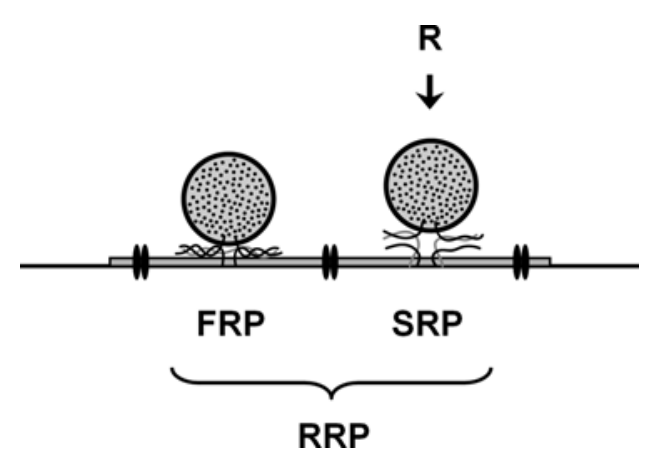

$\mathrm{B} 2$

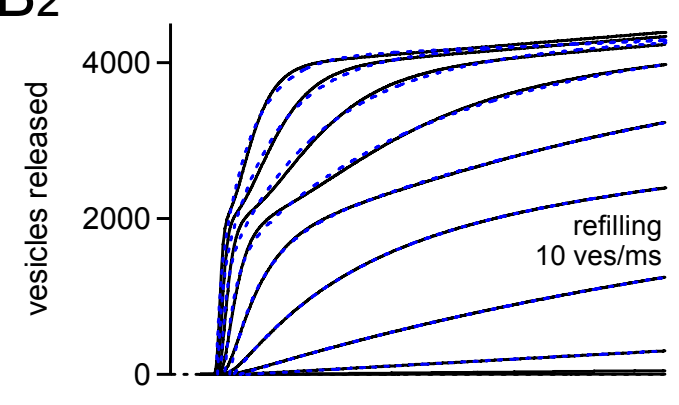

C

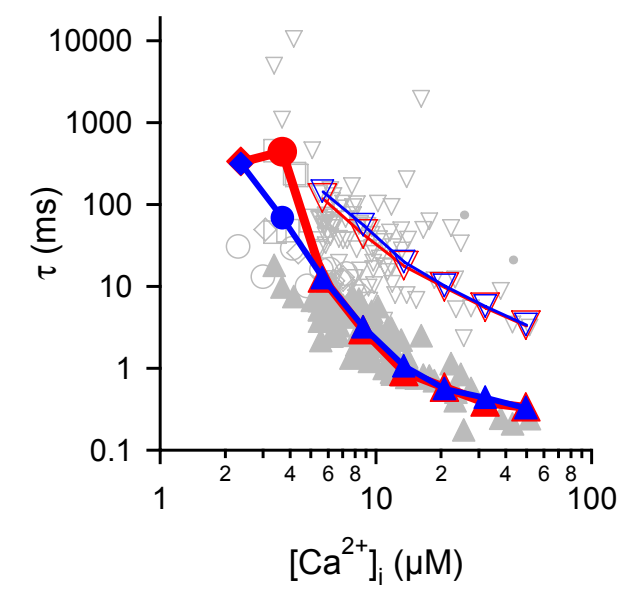

$E$

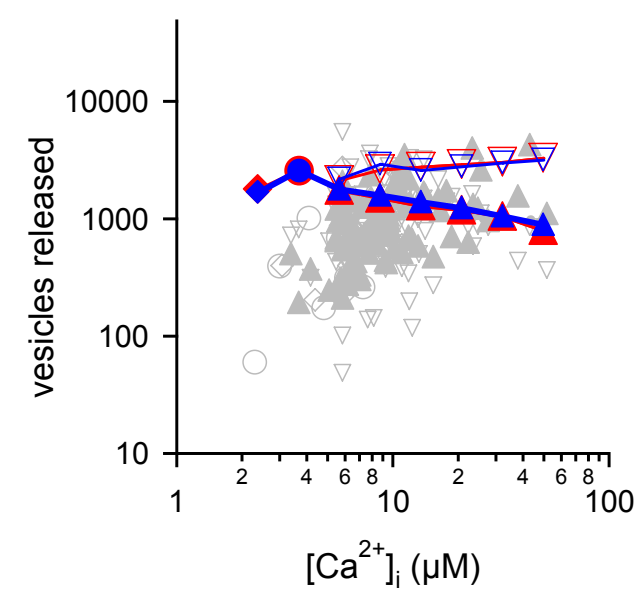

$\mathrm{B}_{1}$

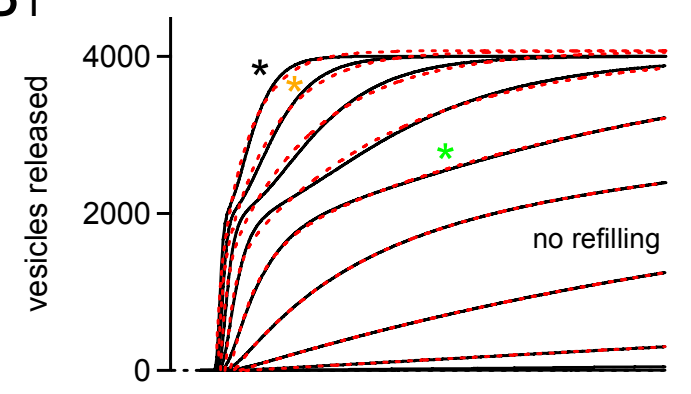

B3

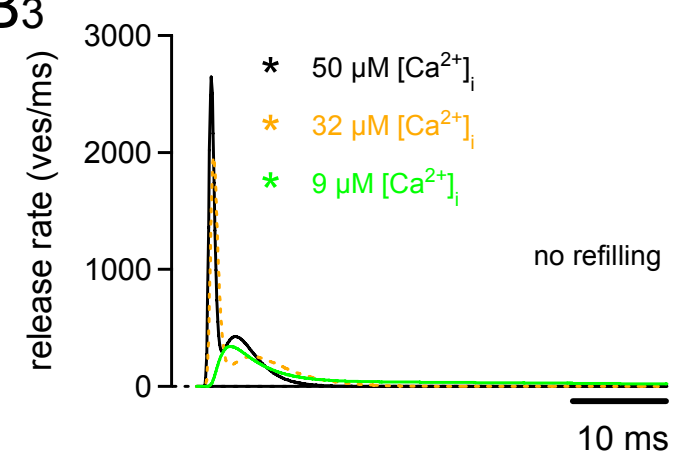

D

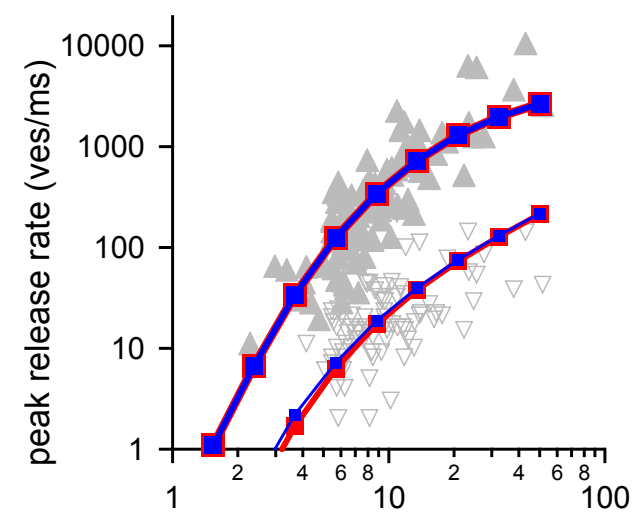

$\left[\mathrm{Ca}^{2+}\right]_{\mathrm{i}}(\mu \mathrm{M})$

$\mathrm{F}$

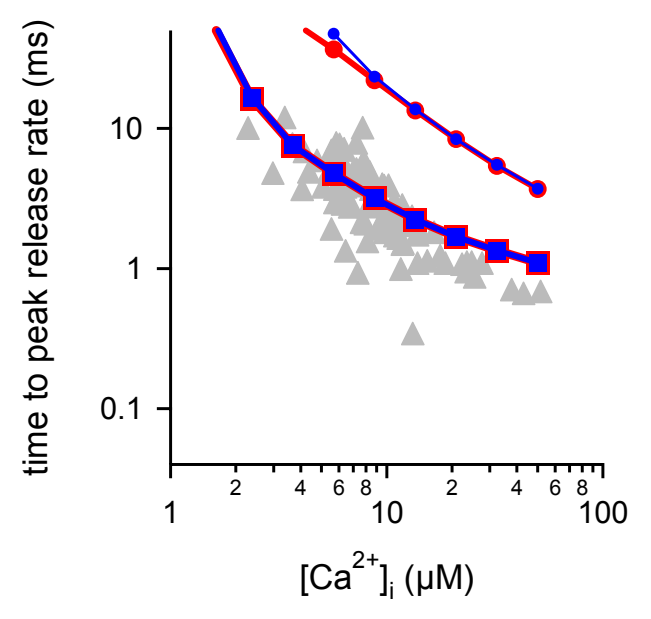




\subsection{A single pool model with intrinsic heterogeneity}

With increasing stimulus strength in terms of $\left[\mathrm{Ca}^{2+}\right]_{\text {, }}$, increasing amounts of fast release at the expense of the slow component were observed in experiments (submaximal release in Fig. 23; chapter 3.4). This indicates that the fast and the slow release component are not mutually independent. Vesicles with an initially high release probability, and thus fast release rates, might convert into slow ones, and this process might directly or indirectly depend on $\left[\mathrm{Ca}^{2+}\right]_{\mathrm{i}}$. This was implemented in a novel single pool model with intrinsic heterogeneity in release kinetics (Fig. 24D), having tested several distinct models to describe both the experimentally observed appearance of two release components and the effect of submaximal release.

The basic concept of this model (Fig. 24D, E) is as follows. There are two states of the vesicle fusion machinery, which includes the $\mathrm{Ca}^{2+}$ sensor (denoted $X$, or $X^{\prime}$ in Fig. 24E). Release can occur from both states, $X$ and $X^{\prime}$, but with a lower rate constant from $X^{\prime}$. With appropriate kinetic parameters, at resting $\left[\mathrm{Ca}^{2+}\right]_{\mathrm{i}}($ about $100 \mathrm{nM})$ almost all vesicles will be in state $X$ with no $\mathrm{Ca}^{2+}$ bound ( $\mathrm{X}_{\mathrm{OCa}}$ in Fig. $24 \mathrm{E}$ ), before a stimulus is applied. When $\left[\mathrm{Ca}^{2+}\right]_{i}$ rises, the $\mathrm{Ca}^{2+}$ sensor will be more occupied with $\mathrm{Ca}^{2+}$ (states $\mathrm{X}_{1 \mathrm{Ca}}, \mathrm{X}_{2 \mathrm{Ca}}, \ldots$ ). At the same time, if the rate of $\left[\mathrm{Ca}^{2+}\right]_{\mathrm{i}}$ rise is slow enough, a sizeable fraction of vesicles might convert into the parallel states $X^{\prime}{ }_{1 \mathrm{Ca}}, X^{\prime}{ }_{2 \mathrm{Ca}}$, etc. (Fig. 24E, brown part), from which the vesicles will be released more 'reluctantly'. Thus the conformational transition from $X$ to $X^{\prime}$ ' (Fig. 24E, from black to brown, respectively) might be seen analogous to the entry into a 'desensitized' state for ligand-activated ion channels. Moreover, this model assumes that release can occur also from the non-fully $\mathrm{Ca}^{2+}$ bound states $\left(X_{0 \mathrm{Ca}}-X_{4 \mathrm{Ca}}\right.$ and $\left.\mathrm{X}^{\prime}{ }_{0 \mathrm{Ca}}-\mathrm{X}^{\prime}{ }_{4 \mathrm{Ca}}\right)$, similar as described recently (Lou et al., 2005), but differing from the 5 -site $\mathrm{Ca}^{2+}$ binding model described above (chapter 4.1; Fig. 24C; Schneggenburger and Neher, 2000). Conversion between the states $X$ and $X^{\prime}$ could occur from every state of $\mathrm{Ca}^{2+}$ binding (Fig. 24E, conversion between $X_{n C a}$ and $X_{n C a}^{\prime}$ with $\left.n=\{1 ; 2 ; 3 ; 4 ; 5\}\right)$, but not from the $\mathrm{Ca}^{2+}$ unbound states $\left(\mathrm{X}_{0 \mathrm{Ca}}\right.$ and $\left.\mathrm{X}^{\prime}{ }_{\mathrm{OCa}}\right)$.

The reaction rates of the model (Fig. 24E) underlie certain theoretical constraints. The range of reasonable transition rates from the fast releasing state $X$ to the slower releasing state $X^{\prime}$ ( $c_{s}$ in Fig. 24E) is limited towards the low end by the extent of submaximal release at relatively low stimulus intensities. Only if the transition rate $c_{s}$ from fast to slow $\left(X\right.$ to $\left.X^{\prime}\right)$ is faster than the release rate from state $X$ at low stimulus intensities, vesicles will be able to 'desensitize' to state $X$ ' before they fuse from state $X$. This behavior is necessary to create a slow release component. On the other hand, the high end of possible transition rates from $X$ to $X^{\prime}$ is limited by release rates from state $X$ at high stimulus intensities, because submaximal release showed the tendency to be overcome by very fast release kinetics at 
high $\left[\mathrm{Ca}^{2+}\right]_{\mathrm{i}}$ (Figs. 21 and 22, black traces). Thus, at high stimulus intensities release must be faster than the assumed transition from the fast releasing state $X$ to the 'desensitized' state $X^{\prime}$, giving an upper limit for 'desensitization' rates. Also the impact of transition rates at basal $\left[\mathrm{Ca}^{2+}\right]_{i}$ is of importance. Without stimulation, the majority of the vesicles should be in the fast releasing state $X$, because if vesicles were initially in the state $X^{\prime}$, fast release rates would be influenced by a necessary transition from $X^{\prime}$ to $X$. Simulations of this scenario, with vesicles accumulated in the 'desensitized' state $X$ ', did not predict fast release rates (not shown) like those which could be observed experimentally. Thus, to keep vesicles in the fast releasing state $X$ at rest, convertibility between the states $X$ and $X^{\prime}$ was omitted for $\mathrm{Ca}^{2+}$ sensors not bound to $\mathrm{Ca}^{2+}\left(\mathrm{X}_{\mathrm{OCa}}\right.$ and $\left.\mathrm{X}^{\prime}{ }_{0 \mathrm{Ca}}\right)$. With this restriction, the fraction of vesicles being in the fast releasing state at rest depends mainly on the ratio between forward-reaction rates and backward-reaction rates from state $X_{0 \mathrm{Ca}}$ to state $\mathrm{X}^{\prime}{ }_{1 \mathrm{Ca}}$. Instead of omitting convertibility between $\mathrm{Ca}^{2+}$ unbound states, also an alternative attempt to keep vesicles in state $\mathrm{X}$ at low $\left[\mathrm{Ca}^{2+}\right]_{i}$ was tried. The ratio of the conversion rates $c_{s}$ and $c_{f}$ for transitions between $\mathrm{X}_{0 \mathrm{Ca}}$ and $\mathrm{X}^{\prime}{ }_{0 \mathrm{Ca}}$ was changed, while leaving the conversion rates between the $\mathrm{Ca}^{2+}$ bound states $\left(\mathrm{X}_{\mathrm{nCa}}\right.$ and $X^{\prime}{ }_{n C a}$ with $\left.n=\{1 ; 2 ; 3 ; 4 ; 5\}\right)$ unchanged. This approach did not succeed, because microscopic reversibility necessitated concomitant changes in the rates of $\mathrm{Ca}^{2+}$ binding or unbinding, so that simulated release could not predict experimental findings anymore (not shown).

Indeed, the empirically obtained kinetic parameters of the model met the criteria mentioned above. The desensitization rate $c_{s}$ was 20 -times faster than the resensitization rate $c_{f}$. Moreover, the omission of convertibility between $\mathrm{Ca}^{2+}$ unbound states ( $\mathrm{X}_{0 \mathrm{Ca}}$ and $\mathrm{X}^{\prime}{ }_{\mathrm{OCa}}$ ) prevented massive 'desensitization' at steady-state conditions with basal $\left[\mathrm{Ca}^{2+}\right]_{i}$ of less than $200 \mathrm{nM}$ (not shown). Fusion rates from the 'desensitized' states ( $\left.X^{\prime}\right)$ were 100-times smaller as compared to the fast releasing states $(X)$. To test for the impact of vesicle recruitment, simulations were run either without refilling, or with a refilling rate of $10 \mathrm{ves} / \mathrm{ms}$ (Fig. 28B1 and B2, respectively). Parameters were: $k_{\text {on }}=1 \cdot 10^{-8} \mathrm{M}^{-1} \mathrm{~s}^{-1}, k_{\text {off }}=4000 \mathrm{~s}^{-1}, a=1, b=0.5, L$ $=2 \cdot 10^{-4} \mathrm{~s}^{-1}, L^{\prime}=2 \cdot 10^{-6} \mathrm{~s}^{-1}, f=40, c_{f}=10 \mathrm{~s}^{-1}, c_{s}=200 \mathrm{~s}^{-1}$, pool size $=4000$ ves.

The predicted time course of release (Fig. 28B) showed a biphasic behavior very similar to that observed in experiments (Figs. 12, 16, 17, 21, 22). The separation between the fast and the slow release component was not as pronounced as compared to predictions from the two pool model with intrinsic heterogeneity (Fig. 27B; chapter 4.3), but predicted time courses of release more similar to experimental findings (compare Fig. 28B and 27B with Figs. 12, 16, $17,21,22)$. 
The predicted $\left[\mathrm{Ca}^{2+}\right]_{i}$ dependence of release time constants, peak release rates, and amounts of vesicles released agreed well with experimental results (Fig. 28C - E), even the shallower dependence of peak release rates of the slow component at $\left[\mathrm{Ca}^{2+}\right]_{i}$ above $15 \mu \mathrm{M}$ could be reproduced (Fig. 28D). Furthermore, the effect of submaximal release (Fig. 28E) was as pronounced as for the experiments (chapter 3.4; Fig. 23), the contribution of the fast component strongly increased at the expense of slow release (Fig. 28B3, compare to Fig. 21 - 22; Fig. 28E, compare open and closed triangles). However, times-to-peak release rate predicted by this model differed more from experimental results as compared to those predicted by the two pool model with intrinsic heterogeneity (Figs. $28 \mathrm{~F}$ and $27 \mathrm{~F}$, respectively). The single pool model with intrinsic heterogeneity showed times-to-peak release rate being less dependent on $\left[\mathrm{Ca}^{2+}\right]_{i}$ than expected.

Simulations without or with refilling (Fig. 28, red and blue data respectively) did not show any significant differences in the kinetic parameters estimated (Fig. 28C - D), thus the analysis method applied could filter refilling from simulated traces satisfyingly.

The same concept was also implemented using the 5 -site $\mathrm{Ca}^{2+}$ binding model with fusion only from the fully $\mathrm{Ca}^{2+}$ bound state $\left(\mathrm{X}_{5 \mathrm{Ca}}\right.$ in Fig. $\left.24 \mathrm{C}\right)$, instead of the model with fusion from every state of $\mathrm{Ca}^{2+}$ binding $\left(\mathrm{X}_{0 \mathrm{Ca}}-\mathrm{X}_{5 \mathrm{Ca}}\right.$ and $\mathrm{X}^{\prime}{ }_{0 \mathrm{Ca}}-\mathrm{X}_{5 \mathrm{Ca}}$ in Fig. 24E). Results from both concepts were similar (not shown), but the latter (Fig. 24E) predicted peak release rates of the slow component, and submaximal release of the fast component more convincingly (not shown). Furthermore, the kinetic scheme shown in Fig. 24E was able to reproduce experimentally obtained low release rates at $\left[\mathrm{Ca}^{2+}\right]_{i}$ below $1 \mu \mathrm{M}$ (not shown), whereas the other model (Fig. 24C) could not (Lou et al., 2005).

Fig. 28 Simulated release: The single pool model with intrinsic heterogeneity

Same display as in Fig. 25, but release was simulated using a single pool model with states of intrinsically different rates of vesicle fusion (A, also in Fig. 24D), as shown in the kinetic scheme in Fig. 24E. Parameters were: $k_{\text {on }}=1 \cdot 10^{-8} \mathrm{M}^{-1} \mathrm{~s}^{-1}, k_{\text {off }}=4000 \mathrm{~s}^{-1}, a=1, b=0.5, L=2$. $10^{-4} \mathrm{~s}^{-1}, L^{\prime}=2 \cdot 10^{-6} \mathrm{~s}^{-1}, f=40, c_{f}=10 \mathrm{~s}^{-1}, c_{s}=200 \mathrm{~s}^{-1}$, pool size $=4000$ ves. Refilling was either omitted (B1, and red data), or accounted for with a rate of $10 \mathrm{ves} / \mathrm{ms}$ (B2, and blue data). B3 Selected release time courses (continuous traces) as in B1 are plotted together with the corresponding fast release components (dotted traces), as obtained from fits (shown in B1, red traces). 

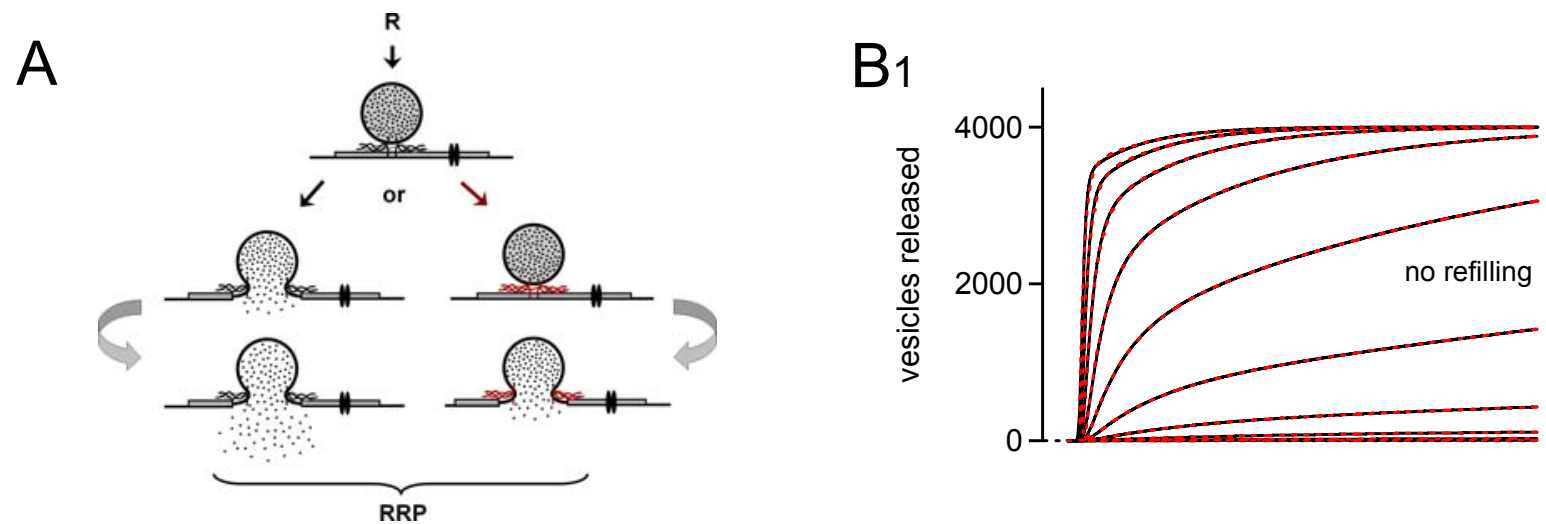

$\mathrm{B} 2$

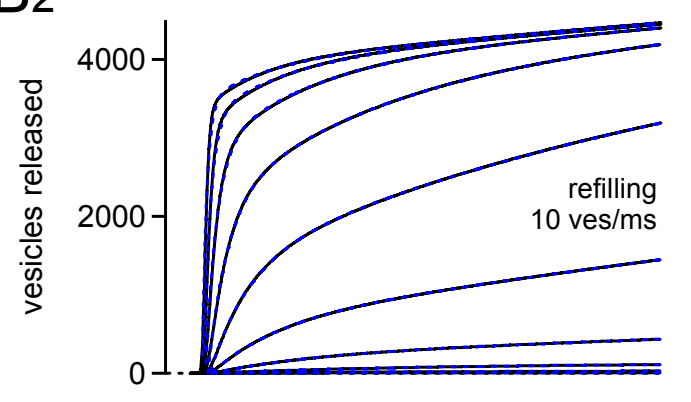

C

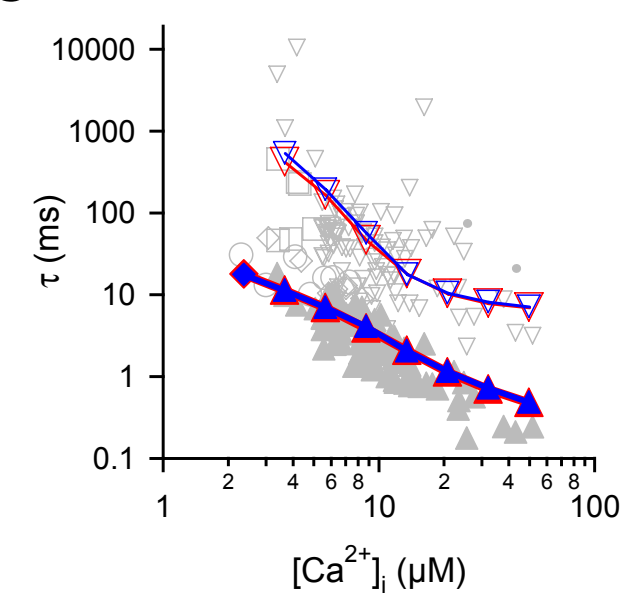

$E$
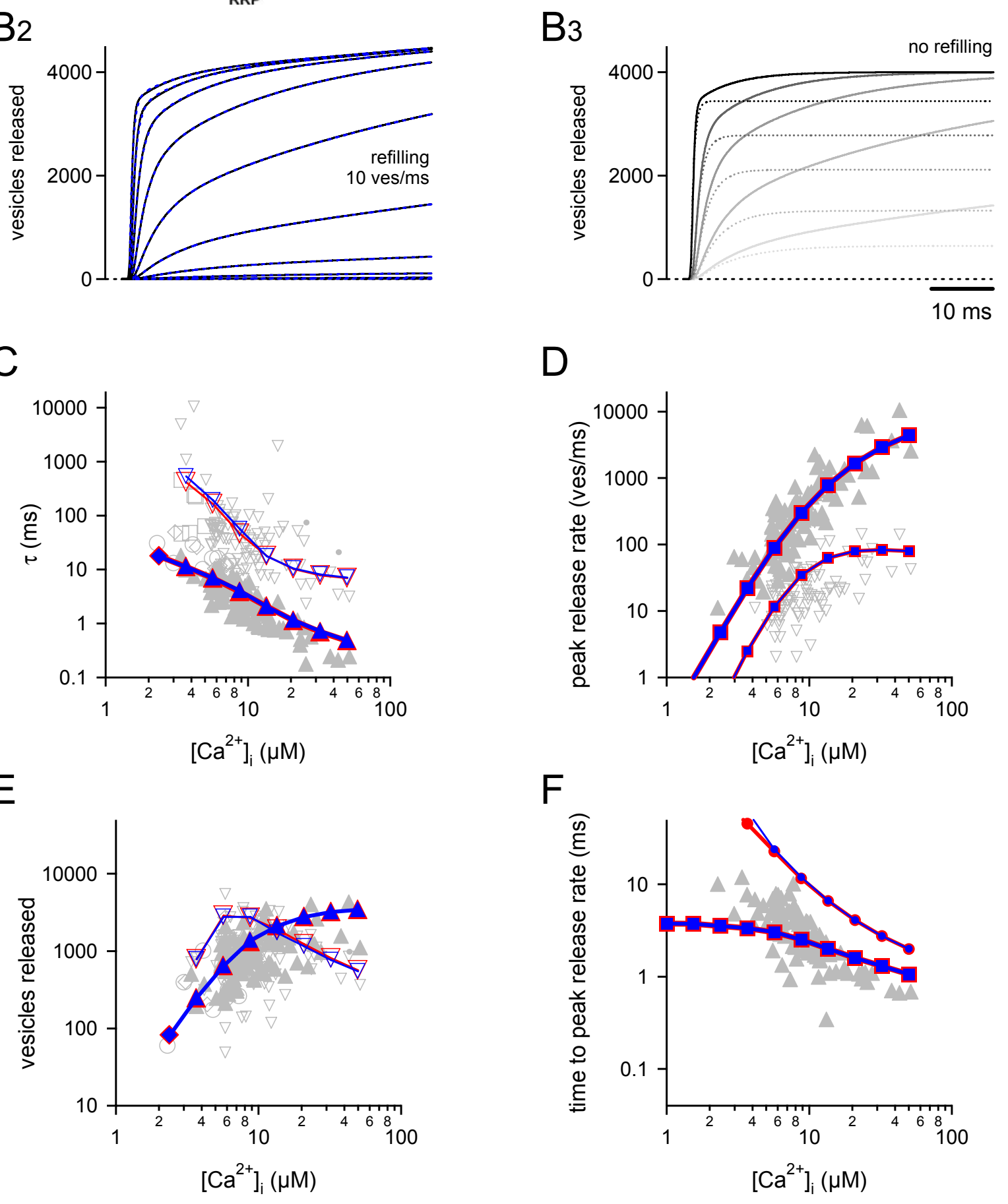

D

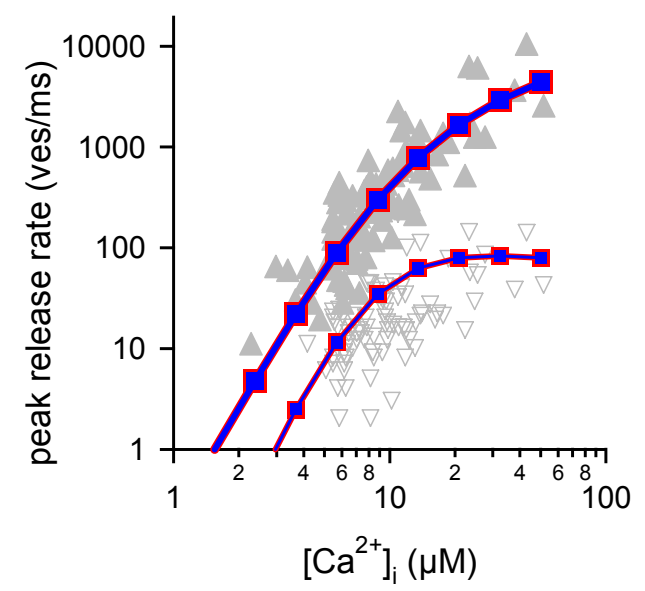

$\mathrm{F}$

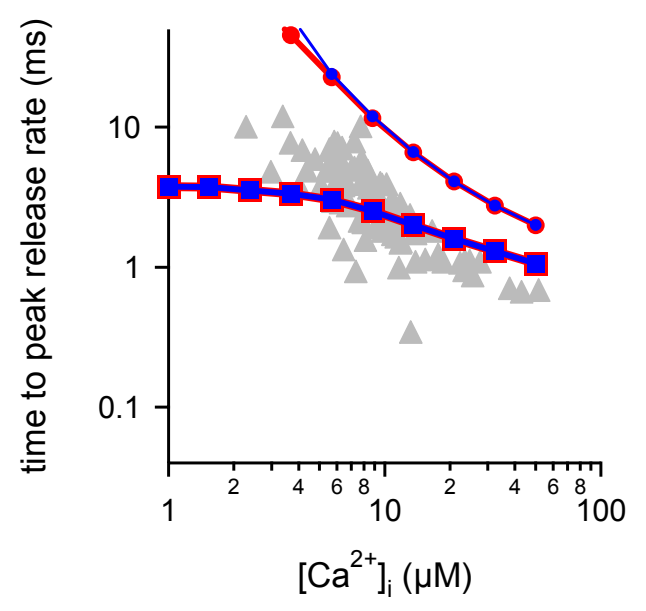




\subsection{Analysis methods also prove for simulated release}

To verify the quality of the analysis methods applied to experimentally obtained time courses of cumulative release, the standard method of fitting several regressions types with a linear refilling component included (see Methods, chapter 2.4.2) was compared to a method of initial refilling correction and subsequent fitting of regressions without a refilling component included (see Methods, chapter 2.4.2). This was done by applying both methods to simulated time courses of cumulative release predicted by the single pool model (chapter 4.1; Fig. 29A1), and other ones resulting from the two pool model with intrinsic heterogeneity (chapter 4.3; Fig. 29A2).

Uncorrected cumulative release traces (Fig. 29; blue traces) together with those after initial refilling correction (red traces) are shown for different stimulus intensities. After classifying each release trace according to its time course, it can be seen that for the single pool model RRP estimates are almost indistinguishable, no matter if an initial refilling correction was done or not (Fig. 29A3 - 4, red and blue symbols, respectively). Fitting to the uncorrected release traces led to a second, slow release component additionally to the RRP estimate at four of seven stimulus intensities (Fig. 29A3, open blue triangles); however, their time constants were too slow to be mistaken for a meaningful release component of the RRP (compare chapter 4.1).

The same conclusion held for simulated traces coming from the two pool model with intrinsic heterogeneity (Fig. 29B). FRP estimates were stable, and independent of the refilling approximation made (Fig. 29B3 - 4). Furthermore, at $\left[\mathrm{Ca}^{2+}\right]_{i}$ above $10 \mu \mathrm{M}$, the resulting parameters for the SRP did not differ either. At $8 \mu \mathrm{M}\left[\mathrm{Ca}^{2+}\right]_{\mathrm{i}}$, the initial refilling correction led to slower SRP release kinetics, and below $8 \mu \mathrm{M}$ even to disappearance of the SRP, that was originally existent in the simulations. This was expected, since at low stimulus intensities SRP kinetics approached those of its own refilling, such that both intermingled. Thus, the initial refilling correction could not distinguish between both anymore, and erased the SRP

Fig. 29 Methods of refilling correction tested on simulated release Same as in Figs. 25 and 27, but analysis results after initial refilling correction (red data; see Methods) are compared to results using the standard method (blue data). Comparisons are made for the single pool model (A; see also Fig. 25), and the two pool model with intrinsic heterogeneity (B; see also Fig. 27). The refilling rate was always set to $10 \mathrm{ves} / \mathrm{ms}$, and resulting cumulative release is shown without the regressions made. 

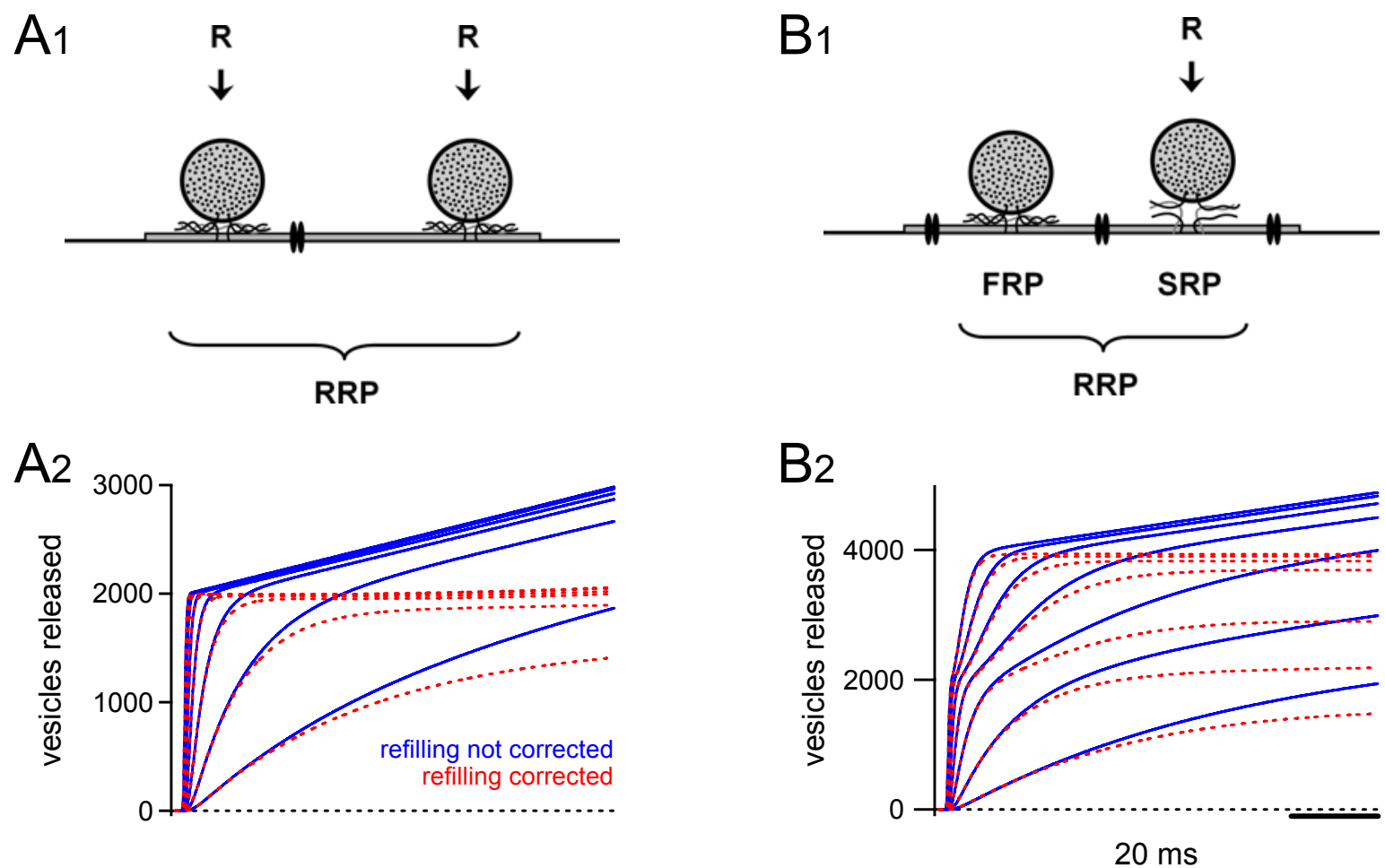
A3-4, B3-4, best fit types:
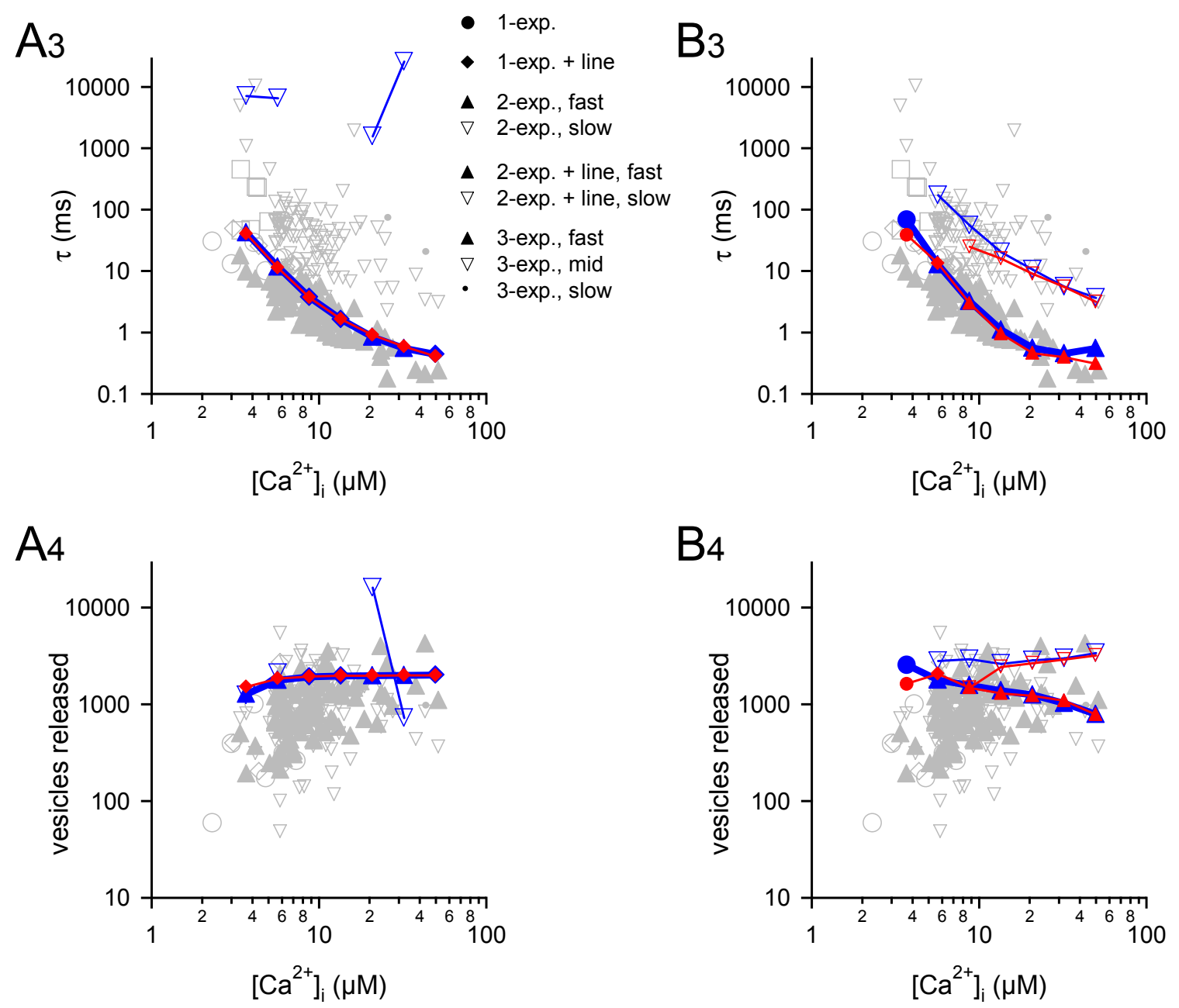
completely. On the other hand, using the standard regression method without initial refilling correction might have led to estimates of SRP release kinetics biased by the dominant kinetics of SRP refilling rather than pure SRP release, at low $\left[\mathrm{Ca}^{2+}\right]_{\mathrm{i}}$. 


\section{Discussion}

This study aimed to clarify the mechanism underlying kinetically heterogeneous release at the calyx of Held synapse in the CNS of rodents. The occurrence of two kinetically distinct release components during presynaptic depolarizations has been reported before at the calyx of Held (Sakaba and Neher, 2001a; Sakaba and Neher 2001b), but also in other CNS synapses (Goda and Stevens, 1994; Hsu and Jackson, 1996; Mennerick and Matthews, 1996; Murthy et al., 1997; Sakaba et al., 1997; von Gersdorff et al., 1998; Yawo, 1999), at neuromuscular junctions (Rahamimoff and Yaari, 1973), and in chromaffin cells (Neher and Zucker, 1993; Horrigan and Bookman, 1994; Seward and Nowycky, 1996; Moser and Neher, 1997; Voets et al., 1999; Voets, 2000). To unravel the mechanism leading to heterogeneous release probabilities, in this study presynaptic $\mathrm{Ca}^{2+}$ uncaging and $\left[\mathrm{Ca}^{2+}\right]_{\mathrm{i}}$ imaging at the calyx of Held was combined with postsynaptic voltage-clamp recordings, and deconvolution of EPSCs (Neher and Sakaba, 2001; Sakaba and Neher, 2001a). Also, $\mathrm{Ca}^{2+}$ uncaging was partially extended by presynaptic depolarizations, and the finding of heterogeneous release probabilities was confirmed with presynaptic $\mathrm{C}_{m}$ measurements as an independent method. Several mechanisms underlying heterogeneous release probabilities at an active zone have been proposed (Neher and Zucker, 1993; Hsu and Jackson, 1996 ; Neher, 1998; Xu et al., 1998; Voets et al., 1999; Xu et al, 1999; Voets, 2000; Sakaba and Neher, 2001a; Sørensen, 2004). The major hypotheses can be separated into two classes: (1) positional heterogeneity, and (2) intrinsic heterogeneity. Positional heterogeneity is based on different distances between voltage activated $\mathrm{Ca}^{2+}$ channels and vesicles, inducing different local $\left[\mathrm{Ca}^{2+}\right]_{\mathrm{i}}$ at release sites, and thus also different release probabilities. Intrinsic heterogeneity includes mechanisms such as (2a) different states of the release apparatus in terms of different priming steps, $(2 b)$ inhibitory effects by previous release on an initially homogeneous release probability, or $(2 c)$ different $\mathrm{Ca}^{2+}$ sensors, or different molecular states of the same $\mathrm{Ca}^{2+}$ sensor, with distinct $\mathrm{Ca}^{2+}$ sensitivities.

Several of the above mentioned models were explored in this study by kinetic simulations of release. Many of the observed release properties, especially submaximal release, could be accounted for by a novel single pool model with intrinsic heterogeneity. The major feature of this mechanism is that a vesicle can fuse with fast kinetics or, after conversion into a reluctant state, with slow kinetics. This effect is comparable to 'desensitization' of the $\mathrm{Ca}^{2+}$ sensor, or an increased energy barrier for the fusion complex to initiate release, leading to a slowed release time course. 


\section{$\mathrm{Ca}^{2+}$ uncaging induces release of the same vesicles as observed by AP-like stimuli}

In this study, the major technique to elicit presynaptic release was $\mathrm{Ca}^{2+}$ uncaging. Increasing the global $\left[\mathrm{Ca}^{2+}\right]_{i}$ in a spatially homogeneous manner for periods of several hundreds of milliseconds is an unphysiological stimulus, that might have induced fusion of vesicle not being triggered for release by APs, e.g. because they were located outside active zones. On the other hand, a global change in $\left[\mathrm{Ca}^{2+}\right]_{\mathrm{i}}$ was aimed to be able to determine the exact $\left[\mathrm{Ca}^{2+}\right]_{i}$ at the $\mathrm{Ca}^{2+}$ sensor for a given stimulus, and furthermore, a step-like $\left[\mathrm{Ca}^{2+}\right]_{\mathrm{i}}$ elevation for 100 ms was needed for a convincing approximation of release kinetics. In control experiments (Fig. 10), release kinetics and release amplitudes yielded by trains of AP-like presynaptic depolarizations under voltage-clamp were in consensus with results from prolonged depolarizations. Trains of AP-like stimuli also released vesicles with a fast and a slow component, and also diminished the response of a subsequent prolonged depolarization (50 ms; Fig. 10A - B). Moreover, these findings were in agreement with results from $\mathrm{Ca}^{2+}$ uncaging experiments (Fig. 14), in the sense that release time constants and release amplitudes of the fast and the slow component had comparable values. Thus, trains of APlike stimuli released vesicles from the same $\mathrm{RRP}$ as $\mathrm{Ca}^{2+}$ uncaging (see also Schneggenburger et al., 1999). Given that the same pool of vesicles was released by $\mathrm{Ca}^{2+}$ uncaging and depolarizations, the similarity of relative release time constants indicates, that $\left[\mathrm{Ca}^{2+}\right]_{\mathrm{i}}$ rises to $10-15 \mu \mathrm{M}$ during a prolonged depolarization to $0 \mathrm{mV}$. Also in other studies, the peak $\left[\mathrm{Ca}^{2+}\right]_{\mathrm{i}}$ in a $\mathrm{Ca}^{2+}$ microdomain around voltage activated $\mathrm{Ca}^{2+}$ channel pores was estimated to be in the range of $10-15 \mu \mathrm{M}$ (Schneggenburger and Neher, 2000; Bollmann et al., 2000; Felmy et al., 2003). Moreover, release time constants and amplitudes obtained from $\mathrm{C}_{\mathrm{m}}$ measurements after presynaptic depolarizations of varying lengths (Fig. 11) matched the estimates from $\mathrm{Ca}^{2+}$ uncaging experiments (Fig. 14). Thus, an identity of vesicles released by APs and by $\mathrm{Ca}^{2+}$ uncaging could be assumed.

\subsection{Two apparent release components based on intrinsic heterogeneity}

\section{The slow release component was not a consequence of changes in $\left[\mathrm{Ca}^{2+}\right]_{i}$}

Elevating $\left[\mathrm{Ca}^{2+}\right]_{i}$ in the presynaptic terminal in a spatially homogeneous manner by $\mathrm{Ca}^{2+}$ uncaging (Fig. 6B) led to two kinetically distinct components of release (Figs. 12, 16, 17, 21, 22). To assure that the appearance of these two release components was not due to experimental or analytical shortcoming, several possible sources of error had to be ruled out. 
First, changes in the $\left[\mathrm{Ca}^{2+}\right]_{i}$ signal might have led to changes in release probabilities. The observed difference in release kinetics for the two components was unlikely to be caused by rapid decreases in $\left[\mathrm{Ca}^{2+}\right]_{\mathrm{i}}$, such as brief $\left[\mathrm{Ca}^{2+}\right]_{\mathrm{i}}$ spikes (Zucker, 1993), which possibly would not have been detected due to a delay of the onset of $\left[\mathrm{Ca}^{2+}\right]_{i}$ measurements after $\mathrm{Ca}^{2+}$ uncaging (4 ms; chapter 2.3). $\left[\mathrm{Ca}^{2+}\right]_{i}$ spikes occur, when uncaged $\mathrm{Ca}^{2+}$ is buffered rapidly by binding to remaining $\mathrm{Ca}^{2+}$ chelator molecules, that were neither occupied with $\mathrm{Ca}^{2+}$ before stimulation, nor were photolysed by the UV stimulus (Zucker, 1993). Such a rebinding effect was not to be expect in this study, since the $\mathrm{Ca}^{2+}$ chelator DMN was bound to $\mathrm{Ca}^{2+}$ to a high degree (chapter 2.3; see also Schneggenburger and Neher, 2000: Fig. 3d). However, if $\left[\mathrm{Ca}^{2+}\right]_{\mathrm{i}}$ spikes still occurred in this study, the initial $\left[\mathrm{Ca}^{2+}\right]_{\mathrm{i}}$ must have dropped at least threefold within $4 \mathrm{~ms}$, to account for a ten-fold difference in release time constants of the fast and the slow component, as observed experimentally (Fig. 14A). Moreover, this drastic change in $\left[\mathrm{Ca}^{2+}\right]_{\mathrm{i}}$ must have ceased already, when $\left[\mathrm{Ca}^{2+}\right]_{\mathrm{i}}$ imaging started, because it could not be detected. In some cases, fast release still continued after onset of $\left[\mathrm{Ca}^{2+}\right]_{\mathrm{i}}$ measurements (e.g. in Fig. 21, yellow and red traces), while a drop in $\left[\mathrm{Ca}^{2+}\right]_{i}$ was not observed. This was most obvious at low $\left[\mathrm{Ca}^{2+}\right]_{\text {, }}$, when the release time constant of the fast component is relatively slow, so that the fast component of release still continued as soon as $\left[\mathrm{Ca}^{2+}\right]_{i}$ could be determined. Furthermore, the estimated dependence of the release probability of the fast component on $\left[\mathrm{Ca}^{2+}\right]_{\mathrm{i}}$ agreed well with results from a previous study, estimating $\left[\mathrm{Ca}^{2+}\right]_{\mathrm{i}}$ already $200 \mu \mathrm{s}$ after $\mathrm{Ca}^{2+}$ uncaging induced by a UV laser pulse (Bollmann et al., 2000). Thus, initial $\left[\mathrm{Ca}^{2+}\right]_{i}$ spikes (Zucker, 1993) could not result in an artificially emerging fast release component.

Second it is possible that a relatively slow decay in $\left[\mathrm{Ca}^{2+}\right]_{i}$ could lead to a reduced release probability, and thus, to the appearance of an artificially distinct, slow release component. But the experimental approach aimed at constant $\left[\mathrm{Ca}^{2+}\right]_{\mathrm{i}}$ for the time range of analysis (Fig. 5 ), and in many recordings, $\left[\mathrm{Ca}^{2+}\right]_{i}$ stayed constant, or even increased slightly (Figs. 5B, 17, 21). Under these conditions, both release components were still observed. Furthermore, simulations based on a decaying $\left[\mathrm{Ca}^{2+}\right]_{\mathrm{i}}$ (Fig. 26) confirmed, that strong $\left[\mathrm{Ca}^{2+}\right]_{\mathrm{i}}$ decays would not result in a second, slower release component with a steep $\left[\mathrm{Ca}^{2+}\right]_{i}$ dependence, as observed experimentally. Therefore, a slow $\left[\mathrm{Ca}^{2+}\right]_{i}$ decay was not the source of the appearance of the slow release component.

\section{Postsynaptic AMPA-receptor saturation and desensitization}

Saturation (Watkins and Evans 1981; Jones et al., 1984) or desensitization (Trussel et al., 1993; Otis et al., 1996; Sun and Wu, 2001) of postsynaptic AMPA-receptors can lead to changes in EPSC time courses. Since presynaptic release time constants were calculated 
from postsynaptic currents, AMPA-receptor saturation or desensitization might have produced an apparent, artificial slow component of release. In the case of AMPA-receptor saturation, the peak release rate of the fast component of release should not have been affected, because it was reached in the rising phase of the EPSC at about half of its maximum. Saturation would affect the peak EPSC, and result in EPSC attenuation, flattening the estimated release time course, and thus lead to an apparent slow release component. However, in all $\mathrm{Ca}^{2+}$ uncaging experiments the competitive AMPA-receptor antagonist $\gamma$-DGG (2 $\mathrm{mM}$ ) was present, and control experiments without and with $\gamma$-DGG led to similar results (Fig. 11), so that an effect by AMPA-receptor saturation could be excluded. In the case of AMPA-receptor desensitization, a similar impairment would have been expected. High concentrations of glutamate in the synaptic cleft can lead to a reduction in the EPSC size, thus artificially decreasing the apparent release probability. But in this study, CTZ was present $(100 \mu \mathrm{M})$ as an blocker of AMPA-receptor desensitization, and furthermore, in control experiments, results from EPSC deconvolution and from independently estimated changes in presynaptic $C_{m}$ both showed two components of release with similar release time constants (Fig. 11). Therefore, an effect of AMPA-receptor desensitization should not have corrupted the data.

In this study, $\mathrm{Ca}^{2+}$ uncaging induced biphasic release (Figs, 12, 1416, 17, 21, 22). All possible artifacts, which could have produced apparent biphasic release, could be excluded, in the sense that $\mathrm{Ca}^{2+}$ uncaging increased $\left[\mathrm{Ca}^{2+}\right]_{i}$ in a spatially homogeneous manner, and temporal changes in $\left[\mathrm{Ca}^{2+}\right]_{\mathrm{i}}$, and AMPA-receptor saturation and desensitization were ruled out to have a marked effect. Furthermore, independent presynaptic $C_{m}$ measurements confirmed the existence of biphasic release. Since $\mathrm{Ca}^{2+}$ channels were not involved during $\mathrm{Ca}^{2+}$ uncaging, positional heterogeneity could be ruled out to be the only mechanism underlying heterogeneous release probabilities, if fast refilling of the RRP did not account for the appearance of the slow component (see below).

Thus, the appearance of two kinetically distinct release components in $\mathrm{Ca}^{2+}$ uncaging experiments, as shown in this study, indicates an intrinsic mechanism underlying biphasic release.

\section{Fast refilling of the RRP does not account for the slow release component}

The slow release component might arise from refilling of the fast component. Fast refilling of the RRP contributes to continuous release during prolonged presynaptic depolarizations at the calyx of Held (Sakaba and Neher, 2001b). In $\mathrm{Ca}^{2+}$ uncaging experiments performed here, 
this refilling component was approximated in the time course of cumulative release either by fitting regressions including a linear component, or by subtracting refilling in a release dependent manner, before regressions for estimates of release kinetics were applied (chapter 2.4.2, and Fig. 8). Neither approximation could erase the slow release component, but slow release showed an exponentially increasing time course of cumulative release (Figs. $8,12,16,17,21,22)$. Also, both analysis methods led to comparable results when tested at simulated release time courses, reproducing predefined parameters convincingly (Fig. 29). Moreover, not only the release time constant of the fast, but also of the slow release component showed a steep dependence on $\left[\mathrm{Ca}^{2+}\right]_{\mathrm{i}}(\mathrm{Fig} .14 \mathrm{~A})$, with release time constants being 10 -fold to 20 -fold slower in a range of $3 \mu \mathrm{M}$ to $50 \mu \mathrm{M}\left[\mathrm{Ca}^{2+}\right]_{\mathrm{i}}$, as compared to fast release. At high $\left[\mathrm{Ca}^{2+}\right]_{i}$ above $20 \mu \mathrm{M}$, time constants of slow release were always below 80 $\mathrm{ms}$, reaching values even below $10 \mathrm{~ms}$. But with a maximal refilling rate of $10 \mathrm{ves} / \mathrm{ms}$ (Sakaba and Neher, 2001b), and a size of the fast release component of about 1500 vesicles (Fig. 14E), a release time constant of refilling slower than $90 \mathrm{~ms}$ can be assumed. Thus, refilling of the fast component and its subsequent release could not account for the slow release component observed, here. Furthermore, simulated release with a fast refilling rate of $10 \mathrm{ves} / \mathrm{ms}$, or an exceedingly fast rate of $100 \mathrm{ves} / \mathrm{ms}$ could not describe the biphasic release time course as observed experimentally, because assuming such high refilling rates essentially adds a linearly rising release component as long as $\left[\mathrm{Ca}^{2+}\right]_{i}$ remains high (Fig. 25). Only the fast release component could be predicted convincingly, whereas slow release due to refilling did not follow the experimentally observed release kinetics, also regarding their $\left[\mathrm{Ca}^{2+}\right]_{i}$ dependence.

These findings indicate that intrinsic heterogeneity is the mechanism underlying heterogeneous release probabilities during $\mathrm{Ca}^{2+}$ uncaging. However, a combination of those factors, which were ruled out to lead to the appearance of the observed slow release component, might still exert an effect different from those caused by a single factor. Such an unlucky combination of negative effects could not be ruled out, but were unlikely.

Release rate estimates independent from EPSC deconvolution also indicated the existence of intrinsic heterogeneity. Analysis of the release time course based on $C_{m}$ measurements unveiled two components of release, as well (Wölfel and Schneggenburger, 2003). However, the temporal resolution was limited, and the signal-to-noise ratio was relatively low in this approach, as compared to EPSC analysis, such that the distinction of two release components became difficult at high $\left[\mathrm{Ca}^{2+}\right]_{i}$ (Wölfel and Schneggenburger, 2003). To further prove the existence of two release components, variance analysis of EPSC noise (Clamann 
et al., 1989; Silver et al., 1998; Reid and Clements 1999; Oleskevich et al., 2000) can be used in $\mathrm{Ca}^{2+}$ uncaging experiments to estimate parameters determining synaptic release, especially because this method was extended to non-stationary release probabilities such as during stimulation (Scheuss and Neher, 2001). Of special interest would be the constancy in quantal size (Scheuss and Neher, 2001) during massive stimulation, such as $\mathrm{Ca}^{2+}$ uncaging or prolonged depolarization. If quantal size changes during stimulation, this might induce a slow release component, although control experiments from this study (Fig. 11) point against changes in quantal size. Unfortunately, variance of EPSC noise analysis could not be done using results from this study, because additional noise due to simultaneous presynaptic $C_{m}$ measurements showed up in the EPSCs, making noise analysis inapplicable for gaining parameters of synaptic transmission.

\section{Characteristics of the fast and the slow release component}

Taken together, these results suggest an intrinsic heterogeneity of release probabilities as the basis of biphasic release kinetics in $\mathrm{Ca}^{2+}$ uncaging experiments. $\mathrm{A}$ fast, and a slow release component exist, differing in their $\left[\mathrm{Ca}^{2+}\right]_{i}$ dependence of release kinetics. In the range of $10 \mu \mathrm{M}$ to $15 \mu \mathrm{M}\left[\mathrm{Ca}^{2+}\right]_{\mathrm{i}}$, being the expected peak $\left[\mathrm{Ca}^{2+}\right]_{\mathrm{i}}$ after a single $\mathrm{AP}$ in the $\mathrm{Ca}^{2+}$ microdomain (Schneggenburger and Neher, 2000; Bollmann et al., 2000), both components were of similar size (about 1500 vesicles), releasing with time constants of about 2 and 40 ms, respectively (Fig. 14). Over the whole $\left[\mathrm{Ca}^{2+}\right]_{i}$ range examined $(3-60 \mu \mathrm{M})$, release time constants of both components showed a power relationship to $\left[\mathrm{Ca}^{2+}\right]_{i}$ of about -1.5 in the double-logarithmic plot (Fig. 14A). A comparable value of 2 was obtained for the power relationship of peak release rates of the fast component relative to $\left[\mathrm{Ca}^{2+}\right]_{i}$ (Fig. 14B). An increase in the power relationship to a maximum of about 4 between 2 and $8 \mu \mathrm{M}\left[\mathrm{Ca}^{2+}\right]_{\mathrm{i}}$ (Schneggenburger and Neher, 2000; Bollmann et al., 2000) was not observed here, since such low stimulus intensities were not tested in detail. However, it was shown that peak release rates had a power relationship to $\left[\mathrm{Ca}^{2+}\right]_{i}$ that decreased at $\left[\mathrm{Ca}^{2+}\right]_{i}$ above $8 \mu \mathrm{M}$, as compared to estimates below $8 \mu \mathrm{M}$ (Schneggenburger and Neher, 2000; Bollmann et al., 2000). For a $\left[\mathrm{Ca}^{2+}\right]_{i}$ range of 5 to $50 \mu \mathrm{M}$, a power relationship of 2 as found in this study (Fig. 14A, B) agreed well with data from previous studies (Schneggenburger and Neher, 2000; Bollmann et al., 2000). In contrast to the fast release component, the power relationship of the peak release rates of the slow component (Fig. 14B) showed a behavior different from that of the corresponding release time constants (Fig. 14A). The apparent $\left[\mathrm{Ca}^{2+}\right]_{\mathrm{i}}$ dependence of peak release rates of the slow component seemed to saturate at $\left[\mathrm{Ca}^{2+}\right]_{\mathrm{i}}$ above $15 \mu \mathrm{M}$. Also, there was a decrease in the number of slowly released vesicles for 
$\left[\mathrm{Ca}^{2+}\right]_{\mathrm{i}}$ above $15 \mu \mathrm{M}$ (Fig. 14E). This phenomenon will be discussed later in the context of submaximal release.

A simple explanation for the appearance of two release components with intrinsically different $\mathrm{Ca}^{2+}$ sensitivities could be the existence of two independent pools of vesicles. This would divide the RRP into an FRP and an SRP, and model calculations showed that the resulting simulated release had comparable kinetics as observed in experiments (Fig. 27). The model was based on a cooperative 5-site $\mathrm{Ca}^{2+}$ binding model (Fig. 24C; Schneggenburger and Neher, 2000), and total release resulted from two such models in parallel. The SRP differed from the FRP by 10 -fold slower on- and off-rates of $\mathrm{Ca}^{2+}$ binding. This approximation was similar to a release model based on results from chromaffin cells (Voets et al, 1999; Voets, 2000), but for the calyx of Held data here, the final fusion rate was unchanged. However, even though analysis of both, real and simulated release time courses, resulted in matching kinetic estimates (Fig. 27), the simulated release time course showed a clear segregation of fast and slow release at high $\left[\mathrm{Ca}^{2+}\right]_{i}$ (Fig. 27B), more pronounced as compared to experimental findings. Also, the increasing amplitude of fast release with increasing $\left[\mathrm{Ca}^{2+}\right]_{\mathrm{i}}$ in the range of $3-10 \mu \mathrm{M}$ was not reproduced by the model (Fig. 27E). Rather, the model predicted that the fast release component should reach maximal amounts of release already at low $\left[\mathrm{Ca}^{2+}\right]_{\mathrm{i}}$ (see closed symbols in Fig. $27 \mathrm{E}$ at $2-4 \mu \mathrm{M}$ ).

\subsection{Intrinsic heterogeneity within a homogeneous pool of vesicles}

\section{Cross-depletion experiments to pre-deplete the fast release component}

To describe the slow release component in more detail, cross-depletion experiments were made, aiming at isolation of the slow release component by pre-depleting the fast releasing vesicles. To do so, short presynaptic depolarizations were given to pre-deplete all fast vesicles, such that only the slow release component would remain for subsequent $\mathrm{Ca}^{2+}$ uncaging. Against the expectations, during the subsequent $\mathrm{Ca}^{2+}$ uncaging still a significant amount of fast vesicles could be released (Figs. 16 -18). Refilling of the fast component was unlikely to account for the appearance of new readily-releasable vesicles, because the time constant of refilling was estimates to be in the range of a few seconds (Sakaba and Neher, 2001b), and here, inter-stimulus intervals were shorter than $50 \mathrm{~ms}$. Rather, incomplete depletion of the fast component by pre-depolarizations took place (Fig. 18D). These observations could be explained by positional heterogeneity of release probabilities for the 
intrinsically fast releasing component. In this case, short depolarizations were too weak to elicit fusion of intrinsically fast vesicles relatively far away from opened $\mathrm{Ca}^{2+}$ channels, and only long depolarizations could lead to complete depletion of the fast release component. In $\mathrm{Ca}^{2+}$ uncaging experiments, high concentrations of the $\mathrm{Ca}^{2+}$ chelator DMN might have increased the effect of local $\left[\mathrm{Ca}^{2+}\right]_{i}$ gradients around activated $\mathrm{Ca}^{2+}$ channels during depolarization, due to strong $\mathrm{Ca}^{2+}$ buffering (Neher, 1998). Indeed, when the situation of strong $\mathrm{Ca}^{2+}$ buffering by DMN (Fig. $16-18$ ) was changed to more moderate $\mathrm{Ca}^{2+}$ buffering, with low concentrations of EGTA as the only $\mathrm{Ca}^{2+}$ buffer present in the presynaptic pipette, higher release efficiencies for the fast component during depolarizations were yielded (Fig. 20). Here, after $16 \mathrm{~ms}$ pre-depolarizations, the fast component was depleted in four out of five cases, and potently reduced in the fifth example. Thus, positional heterogeneity in addition to intrinsic heterogeneity might exist at the calyx of Held synapse. In cross-depletion experiments combined with $\mathrm{Ca}^{2+}$ uncaging, about half of the fast component could not be depleted by short pre-depolarizations, being an upper estimate of the contribution of positional heterogeneity, because of the discussed high buffering conditions by DMN, that enhance the effect of local $\mathrm{Ca}^{2+}$ microdomains. The estimate of maximally $50 \%$ contribution by positional heterogeneity to fast release is comparable to estimates of about $25 \%$ in chromaffin cells (Voets et al, 1999).

Therefore, possibly both, positional and intrinsic heterogeneity contribute to heterogeneity in release probabilities. If intrinsic heterogeneity, based on two independent vesicle pools with different $\mathrm{Ca}^{2+}$ sensitivities, is combined with positional heterogeneity, the total amount of fast vesicles released should have been the same for a single $\mathrm{Ca}^{2+}$ uncaging stimulus as compared to a combination of pre-depolarization and subsequent $\mathrm{Ca}^{2+}$ uncaging. The average data did not show a difference in total fast release, indeed (Fig. 18D), but in some cases, an increased amount of fast release was observed for the cross-depletion approach (Figs. 16 - 17). This was unexpected, because rapid recruitment of new fast releasable vesicles was unlikely, as stated above. Neither different $\mathrm{Ca}^{2+}$ sensitivities of two independent vesicle pools, nor positional heterogeneity, nor a combination of both could explain this phenomenon. Rundown of release was not the origin of less amounts of fast release for the $\mathrm{Ca}^{2+}$ uncaging stimulus (Fig. 17, see temporal order of stimuli applied). The mismatching amounts of fast release will be discussed and clarified below in the context of submaximal release. 


\section{Submaximal release of the fast component at low, constant $\left[\mathrm{Ca}^{2+}\right]_{i}$}

Several observations mentioned before indicated submaximal release of the fast release component, in the sense that depletion of fast releasing vesicles could be incomplete, even when the $\mathrm{Ca}^{2+}$ stimulus persisted. First, pool size estimates for the fast component were smaller at $\left[\mathrm{Ca}^{2+}\right]_{i}$ below $10 \mu \mathrm{M}$ as compared to $10-15 \mu \mathrm{M}$ (Fig. 14E). Second, in crossdepletion experiments combining short pre-depolarizations with subsequent $\mathrm{Ca}^{2+}$ uncaging, some recordings showed that the total amount of fast release after paired stimulation was larger than after a single control $\mathrm{Ca}^{2+}$ uncaging stimulus (Fig. 16 and 17, compare red to black traces, respectively). Fast refilling of the fast release component being the cause of this phenomenon was unlikely, as discussed above. However, the phenomenon of increased amounts of fast vesicles released in response to paired stimulation was not observed in all cases recorded (Fir. 18D). But in similar cross-depletion experiments, this time combining a short pre-depolarization with a subsequent long depolarization, and with $0.2 \mathrm{mM}$ EGTA instead of $1.5 \mathrm{mM}$ DMN presynaptically, the effect of increased cumulative fast release due to paired stimulation as compared to a single pulse was more consistent (Figs. 19, 20C). Furthermore, a tendency of reduced amounts of slow release in parallel to the described increase in fast release could be observed (Fig. 20A), even though the effect was small. It seemed, as if stronger stimulation led to more potent release of fast vesicles at the expense of the slow release component.

A direct experimental approach to unveil the characteristics of submaximal release was made, applying several $\mathrm{Ca}^{2+}$ uncaging stimuli of different intensities within a given cell (examples shown in Figs. 21 - 22). As already indicated in the cross-depletion experiments (Fig. 20), here again the fast component seemed to increase with $\left[\mathrm{Ca}^{2+}\right]_{\mathrm{i}}$ at the expense of the slow release component (Fig. 23). In the range of $3-45 \mu \mathrm{M}$, the cumulative amount of fast release rose significantly from $65 \%$ at $3-10 \mu \mathrm{M}$ to about $143 \%$ at 20 to $45 \mu \mathrm{M}$, as normalized to the range of $10-15 \mu \mathrm{M}\left[\mathrm{Ca}^{2+}\right]_{i}$ (Fig. 23B). The decrease of amounts of slow release from $152 \%$ to $68 \%$ in the same $\left[\mathrm{Ca}^{2+}\right]_{\mathrm{i}}$ ranges was as pronounced as the increase of cumulative fast release, but statistically less significant. This effect of inverse proportionality might be explained by a conversion from slow vesicles to fast vesicles that is enhanced by stronger stimulation, or by a conversion from fast to slow vesicles being reduced by stronger stimulation.

Submaximal vesicle fusion has been reported before at the squid giant synapse (Hsu et al., 1996) and in eggs from sea urchins (Blank et al., 1998a, 1998b). Hsu et al. (1996) showed that the release probability was reduced, and release stopped, before pool depletion was complete. They proposed that $\mathrm{Ca}^{2+}$-dependent adaptation of the release machinery leads to 
conversion into a non-releasing state, thus also leading to monophasic release time courses. In the study by Blank et al. (1998a; 1998b), the mechanism underlying submaximal release was proposed to be a difference in fusion thresholds for different subpopulations of vesicles, being dependent on $\left[\mathrm{Ca}^{2+}\right]_{\mathrm{i}}$ at the release site. The release probability was suggested to have a Gaussian distribution over the indicated range of $\left[\mathrm{Ca}^{2+}\right]_{i}$ thresholds, thus leading to a sigmoidal relationship between fractional pool depletion and stimulus intensity. The rate of fusion itself was $\mathrm{Ca}^{2+}$ dependent, as well, but was suggested to be the same for all subpopulations of vesicles. In the examples shown there, release seemed to be monophasic, but detailed analysis of the time course of release was not made. Thus, at first, the proposed mechanism of release thresholds would not explain the occurrence of a second, slower release component as observed at the calyx of Held. In their model, increasing the stimulus intensity could explain the increasing amount of vesicles released, but at a given $\left[\mathrm{Ca}^{2+}\right]_{\text {, }}$, the release kinetics should be the same for all vesicles fusing. Thus, release would always be kinetically homogeneous at a given stimulus strength, contrasting the biphasic time course of cumulative release, as experimentally obtained at the calyx of Held in this study (Figs. 12, 16, $17,21,22)$.

As stated above, the appearance of two release components could be predicted by a two pool model with intrinsic heterogeneity (Figs. 24B - C, 27; chapter 4.3). But this model could not reproduce the phenomenon of submaximal release. On contrary, the cumulative amount of release seemed to have the opposite dependence on $\left[\mathrm{Ca}^{2+}\right]_{i}$ (Fig. 27E), as compared to experimental results (Fig. 23). In the prediction of the two pool model, amounts of fast release apparently decreased with higher $\left[\mathrm{Ca}^{2+}\right]_{\text {, }}$, while slow release amounts seemed to increase (Fig. 27E). But since the pool sizes of both, FRP and SRP, were kept constant throughout the simulations, the inconsistent estimates for pool sizes must have been an artifact of improper fitting, also obvious from comparison of the simulated release time courses with the corresponding fits (Fig. 27B). Moreover, the pronounced segregation between the fast and slow release component predicted by the model (Fig. 27B) was not observed experimentally (Figs. 12, 16, 17, 21, 22). Thus, the two pool model with intrinsic heterogeneity (Figs. 24B $-\mathrm{C}, 27$ ) predicted release time constant estimates for the fast and the slow release component comparable to those obtained in experiments, but predicted amounts of release differed markedly from experimental results, and could not reproduce the effect of submaximal release.

Therefore, other models were tested to account for both, biphasic release kinetics, and submaximal release. A single pool model with intrinsic heterogeneity (Fig. 24D - E) met these criteria (Fig. 28), as described in the next section. 


\section{A single pool model with intrinsic heterogeneity}

The apparent conversion of the slow release component to the fast one at increasing $\left[\mathrm{Ca}^{2+}\right]_{\mathrm{i}}$ demands for a release model that incorporates mutual dependence of the two release components. A simple explanation is the existence of two inter-convertible states of the release machinery, one leading to fast release kinetics, the other one to slower kinetics, and the release apparatus being able to switch between these two states. This was implemented in a novel scheme as shown in Fig. $24(D-E)$, with fast release from state $X$, and slow release from state $X^{\prime}$ (Fig. 24E, black and brown part, respectively), and conversion possible from every state with at least one $\mathrm{Ca}^{2+}$ ion bound $\left(X_{1 \mathrm{Ca}}-X_{5 \mathrm{Ca}}\right.$ and $\left.X^{\prime}{ }_{1 \mathrm{Ca}}-X^{\prime}{ }_{5 \mathrm{Ca} a}\right)$. The fusion rate from the 'reluctantly' releasing state $X$ ' was 100 -times smaller as compared to the fast releasing state $X\left(L\right.$ and $L^{\prime}$ in Fig. 24E, respectively), and this was the only difference in kinetic parameters between $X$ and $X^{\prime}$. The convertibility between the states $X$ and $X^{\prime}$ is similar to that proposed by Hsu et al. (1996), but in the new model introduced here, fusion from the 'desensitized' state $X$ ' was still possible.

The single pool model with intrinsic heterogeneity (Fig. 24D - E) predicted time courses of cumulative release (Fig. 28B) very similar to those observed experimentally (Figs. 12, 16, 17, $21,22)$. Moreover, estimates for release time constants, peak release rates, and amounts of release agreed well with findings from experiments (Fig. 28C - E). Submaximal release in simulations showed a dependence on $\left[\mathrm{Ca}^{2+}\right]_{\mathrm{i}}$ (Figs. 28B3, E), comparing well to experimental results (Fig. 21 - 23), with an equal contribution to cumulative release of about $50 \%$ from both, the fast and the slow component in the range of $10-15 \mu \mathrm{M}\left[\mathrm{Ca}^{2+}\right]_{\mathrm{i}}$ (Figs. 18C, 28E; see also Sakaba and Neher, 2001b). Furthermore, reduced amounts of slow release at relatively high $\left[\mathrm{Ca}^{2+}\right]_{i}$ led to an apparently reduced $\left[\mathrm{Ca}^{2+}\right]_{i}$ dependence of the peak release rates of the slow component for both, experimental findings and simulations (Fig. 28D, [Ca $\left.{ }^{2+}\right]_{i}$ $>10 \mu \mathrm{M})$. Only the times to peak release rate of the fast component were not predicted by the model accurately (Fig. 28F), and showed a shallower dependence on $\left[\mathrm{Ca}^{2+}\right]_{\mathrm{i}}$, but, however, were still in the right range.

Submaximal release might also be tested with a different approach. In experiments using paired depolarizations, different stimulus intensities leading to different release rates can be elicited by changing the extracellular $\mathrm{Ca}^{2+}$ concentrations from e.g. $0.5 \mathrm{mM}$ up to $15 \mathrm{mM}$ (Meyer et al., 2001). With the release model introduced here, the contribution of fast release to total should increase with an increasing extracellular $\mathrm{Ca}^{2+}$ concentration.

For the squid giant synapse, Hsu et al. (1996) showed that the speed of EPSC decay was independent of $\left[\mathrm{Ca}^{2+}\right]_{\mathrm{i}}$. They concluded that the time constant of vesicle depletion was 
independent of $\left[\mathrm{Ca}^{2+}\right]_{\mathrm{i}}$, as was also predicted by their model of release adaptation. This contrasts with findings from the calyx of Held synapse (Fig. 21 - 22). Here, the EPSC decay accelerated with increasing $\left[\mathrm{Ca}^{2+}\right]_{\mathrm{i}}$ (Figs. $\left.21-22\right)$, caused by the steep $\left[\mathrm{Ca}^{2+}\right]_{\mathrm{i}}$ dependence of the fast and the slow release component (Fig. 14). Thus, the previously proposed conversion into a non-fusing state (Hsu et al., 1996) that predicts $\left[\mathrm{Ca}^{2+}\right]_{i}$ independent EPSC decay times, cannot account for the observations at the calyx of Held, whereas release from a 'desensitized' state, as proposed here, could.

As shown before in cross-depletion $\mathrm{Ca}^{2+}$ uncaging experiments, pre-depolarizations failed to completely eliminate the fast release component, and thus to isolate the slow component for a subsequent pulse (Figs. $16-18$ ). This might be expected, if a conversion from state $X$ to $X^{\prime}$ withholds vesicles from rapid pool depletion, and if a considerable amount of fast release converts back into state $\mathrm{X}$ between the two stimuli. However, this 'resensitization' would have to be relatively fast to exert a detectable effect in between two stimuli separated by only 30 $50 \mathrm{~ms}$, as for the cross-depletion approach shown (Fig. 16 -17). For the release mechanism suggested here, experiments describing recovery from vesicle pool depletion (Sakaba and Neher, 2001b) indicate a rather slow 'resensitization' rate. Recovery from vesicle pool depletion was shown to occur in two phases (Sakaba and Neher, 2001b), with fast refilling of the slowly releasing component, and slower refilling rates for the fast releasing component. The existence of biphasic recovery from depletion (Sakaba and Neher, 2001b) might be explained by the single pool model with intrinsic heterogeneity introduced here. If the basal $\left[\mathrm{Ca}^{2+}\right]_{\mathrm{i}}$ in between the two stimuli was increased to a few hundred $\mathrm{nM}$, vesicles initially being refilled into the fast releasing state $X$ should soon have converted into the slower releasing state $X^{\prime}$. Thus, release in response to the second pulse would be dominated by slower release kinetics, and the slow release component would apparently be refilled faster. But for a more precise examination of the 'resensitization' rate from state $X$ ' to state $X$, additional experiments have to be made. Paired $\mathrm{Ca}^{2+}$ uncaging stimuli can be performed similar to the paired depolarization approach by Sakaba and Neher (2001b), and $\left[\mathrm{Ca}^{2+}\right]_{i}$ in between these stimuli can be measured with a high affinity $\left[\mathrm{Ca}^{2+}\right]_{i}$ indicator dye, such as Fura-2 or Fura-4F. These results then can be compared to predictions by the model, and presumably, the 'resensitization' rate from $X^{\prime}$ to $X$ will have to be adjusted accordingly. Also, the described effect of Calmodulin blockers on RRP refilling (Sakaba and Neher, 2001b) should be included in the experiments. A Calmodulin dependent modulation of the 'resensitization' from state $X^{\prime}$ to $X$ might bring the experimentally observed biphasic recovery that includes a $\mathrm{Ca}^{2+}$ dependent component (Sakaba and Neher, 2001b) in accordance with the release model proposed here. 
Furthermore, the suggested conversion from the fast to the slow release component can be tested by paired stimuli applied to the presynaptic terminal, while monitoring the basal $\left[\mathrm{Ca}^{2+}\right]_{\text {i }}$. With $\mathrm{Ca}^{2+}$ uncaging, the basal $\left[\mathrm{Ca}^{2+}\right]_{i}$ can initially be increased to levels of a few hundred $\mathrm{nM}$ up a few $\mu \mathrm{M}$. This should drive vesicles in the 'reluctantly' releasing state $X^{\prime}$ ' and change release kinetics accordingly, as quantifiable in response to a subsequent $\mathrm{Ca}^{2+}$ uncaging stimulus to higher $\left[\mathrm{Ca}^{2+}\right]_{\mathrm{i}}$ levels.

However, findings from this study already strongly indicate the existence of a single vesicle pool with intrinsic heterogeneity as the underlying mechanism for experimentally observed biphasic release time courses, and also for the steep $\left[\mathrm{Ca}^{2+}\right]_{i}$ dependence of both, the fast and the slow release component, and for submaximal release of the fast component at relatively low $\left[\mathrm{Ca}^{2+}\right]$.

\section{Comparing the new release model with previous findings}

Short-term lateral inhibition in release by membrane incorporation of recently fused neighboring vesicles was proposed as a mechanism leading to reduced release probability (Walmsley et al., 1988; Stevens and Wang, 1995). Membrane incorporation of fused vesicles was thought to result in membrane relaxation and a concomitant increase in the energy barrier for further vesicle fusion. Such a mechanism is unlikely to account for the occurrence of the slower release component, as observed here, because submaximal fast release should not have been overcome by high $\left[\mathrm{Ca}^{2+}\right]_{\mathrm{i}}$, as observed experimentally (Figs. 21 - 23), but would rather be solely dependent on amount of previous release. Only a delayed effect of lateral inhibition cannot be excluded, being slow enough to be overcome by fast fusion rates at high $\left[\mathrm{Ca}^{2+}\right]_{\mathrm{i}}$, and fast enough to induce a slow release component at low release rates at low $\left[\mathrm{Ca}^{2+}\right]_{\text {. }}$.

Bellingham and Walmsley (1999) proposed a high-affinity $\mathrm{Ca}^{2+}$ sensor to induce inhibition of fast release at elevated $\left[\mathrm{Ca}^{2+}\right]_{\mathrm{i}}$, assuming a molecular site directly involved in regulating the vesicular release probability. This process was proposed to be $\left[\mathrm{Ca}^{2+}\right]_{i}$ dependent, such that increased $\left[\mathrm{Ca}^{2+}\right]_{i}$ leads to increased inhibition of release. However, a molecular candidate was not discussed, and two kinetically distinct components of release were not found. Principally, for the mechanism introduced here, the 'desensitization' from state $X$ to $X$ ' might be $\left[\mathrm{Ca}^{2+}\right]_{i}$ dependent, but this dependency was not necessary to predict the experimentally observed release kinetics (Figs. 24E, 28). Rather, the $\left[\mathrm{Ca}^{2+}\right]_{i}$ dependence of submaximal release was an intrinsic property of the model suggested in this study. The absence of an additional special $\mathrm{Ca}^{2+}$ binding site for 'desensitization', and the ability to predict two 
components of release favor the single pool model with intrinsic heterogeneity introduced here.

In chromaffin cells, also two components of release have been observed (Neher and Zucker, 1993; Horrigan and Bookman, 1994; Seward and Nowycky, 1996; Moser and Neher, 1997; Voets et al., 1999; Voets, 2000; Sørensen, 2004). A release mechanism was proposed (Voets et al., 1999; Voets, 2000), in which a slowly releasing component is a fusioncompetent precursor of the fast component during vesicular priming. Thus, in their model, a reversible conversion between a fast and a slowly releasing state exists, similar to the model introduced here, and for both models, conversion is independent of $\left[\mathrm{Ca}^{2+}\right]_{i}$. But the model proposed for release in chromaffin cells (Voets et al., 1999; Voets, 2000) differs from the model introduced in this study (Fig. 24D - E) in three aspects. First, in the chromaffin cell model, fusion can only occur from a $\mathrm{Ca}^{2+}$ sensor fully occupied by $\mathrm{Ca}^{2+}$. But this cannot account for the rather $\left[\mathrm{Ca}^{2+}\right]_{\mathrm{i}}$ invariant release rates at $\left[\mathrm{Ca}^{2+}\right]_{\mathrm{i}}$ below $1 \mu \mathrm{M}$ in the calyx of Held, as shown by Lou et al. (2005). Second, in the chromaffin cell model, conversion between the fast and the slow release component could occur from every state of $\mathrm{Ca}^{2+}$ binding, including the $\mathrm{Ca}^{2+}$ unbound state. The slowly releasable pool in chromaffin cells was predicted to have a similar size as the rapidly releasing pool, always being completely refilled at rest without stimulation. But findings of submaximal release at the calyx of Held synapse (Fig. $21-23$ ) indicate reduced amounts of slow release at high $\left[\mathrm{Ca}^{2+}\right]_{\mathrm{i}}$, and this speaks against the assumption that a large fraction of vesicles exists in a slowly releasing state at rest. Assuming very fast transition rates from the slow to the fast release component cannot solve this problem, because then slow release itself would become unlikely. The third difference between the two models exists in the kinetic reactions that were assumed to account for biphasic release. At the calyx of Held model (Fig. $24 D-E)$, only the final fusion rates ( $L$ and $L^{\prime}$ ) differed between the fast and the slow release component. But for the chromaffin cell model, on- and off-rates of $\mathrm{Ca}^{2+}$ binding, and the fusion rate for the slow release component were decreased ten-fold, as compared to the fast component (Voets, 2000). The transition from a slowly releasing to a rapidly releasing state was suggested to reflect the degree of priming of the SNARE complex, switching from a loose cis-SNARE formation to a tight transSNARE formation, respectively. But differences in the on- and off-rates of $\mathrm{Ca}^{2+}$ binding, and in the final fusion rate, accounting for two components of release in the chromaffin cell model, would most likely also involve the $\mathrm{Ca}^{2+}$ sensor directly, not only the SNARE complex. A bare effect of priming would probably not change on- and off-rates of $\mathrm{Ca}^{2+}$ binding to the $\mathrm{Ca}^{2+}$ sensor, but rather influence only the final fusion rate, as implemented in the single pool 
model with intrinsic heterogeneity introduced here. However, the molecular mechanisms cannot be unveiled on the basis of the biophysical data presented here.

In chromaffin cells, submaximal release was not observed, and the contribution of the fast and the slow release component was independent of $\left[\mathrm{Ca}^{2+}\right]_{i}$ after $\mathrm{Ca}^{2+}$ uncaging (Voets, 2000), and the proposed release model did not account for $\left[\mathrm{Ca}^{2+}\right]_{i}$ dependent submaximal release. But for the calyx of Held, an appropriate release model has to predict the experimentally observed submaximal release as well, and the single pool model with intrinsic heterogeneity introduced here, satisfies this requirement.

After pre-stimulation, slowing of the fast release component has been observed (Figs. 16, 17, 18A, 19, 20B1), which confirms findings by $\mathrm{Wu}$ and Borst (1999). They demonstrated that after rapid recovery from depression, release probability was initially reduced, and recovered within about $30 \mathrm{~s}$. In cross-depletion experiments performed in this study (chapter 3.3), the release kinetics slowed from about $2 \mathrm{~ms}$ in control to about $3 \mathrm{~ms}$ after pre-stimulation, at 10 $\mu \mathrm{M}$ to $15 \mu \mathrm{M}\left[\mathrm{Ca}^{2+}\right]_{\mathrm{i}}$ (Fig. 18A). The single pool model with intrinsic heterogeneity (Figs. $24 \mathrm{D}$ $-E, 28$ ) might explain this effect. At rest, almost all vesicles are in the fast releasing state $X$, as stated above. After a stimulus that partially depletes the RRP, basal $\left[\mathrm{Ca}^{2+}\right]_{i}$ will be elevated, presumably by a few hundred $\mathrm{nM}$, and unreleased vesicles will be bound to $\mathrm{Ca}^{2+}$ more likely. From these $\mathrm{Ca}^{2+}$ bound states $\left(\mathrm{X}_{1 \mathrm{Ca}}, \mathrm{X}_{2 \mathrm{Ca}}, \ldots\right)$, vesicles will tend convert to the slower releasing state $X^{\prime}$, joining those vesicles that entered the state $X^{\prime}$ already during prestimulation, and that did not fuse yet. Thus, when a subsequent, second stimulus is applied, a large fraction of the remaining fusion competent vesicles will be in the slower releasing state $X^{\prime}$, and the contribution of fast release to total release during this second stimulus will be reduced as compared to an unconditioned stimulus, i.e. without pre-stimulation. This reduced fraction of fast release, and concomitant increase of the slowly releasing fraction, can decrease the apparent release rate of the fast component: if release rates are distributed over the range of possible release rates $\left(X^{\prime}{ }_{0 \mathrm{Ca}}\right.$ and $\mathrm{X}_{5 \mathrm{Ca}}$ for the slowest and fastest release rate, respectively), the apparent release time course will show a biphasic rise after stimulation, with apparent fast and slow release rates being intermediate values in the range of effective release rates (verified by simulations, not shown). Thus a reduced fraction of fast release, e.g. due to pre-stimulation and partial conversion to state $X^{\prime}$, will shift release towards lower rates, and the apparent fast release will seem to become slower. The ability of the single pool model with intrinsic heterogeneity to account for slowing of fast release after pre-stimulation could be confirmed in preliminary simulations (not shown), but for a quantitative description, more experiments have to be done. Using paired $\mathrm{Ca}^{2+}$ uncaging 
stimuli, slowing of the fast release rate can be examined quantitatively. The first $\mathrm{Ca}^{2+}$ uncaging stimulus can elevate $\left[\mathrm{Ca}^{2+}\right]_{i}$ to a few $\mu \mathrm{M}$, such that a large fraction of the RRP converts into the 'desensitized' state $X^{\prime}$ ', and in a subsequent, second $\mathrm{Ca}^{2+}$ uncaging stimulus slowing of release time constants can be analyzed.

Wu and Borst (1999) suggested that rapid recovery from pool depletion, and therefore also recovery from the observed reduced release probability, was independent of $\left[\mathrm{Ca}^{2+}\right]_{\mathrm{i}}$. Sakaba and Neher (2001b) showed that rapid recovery of the slow release component is $\left[\mathrm{Ca}^{2+}\right]_{\mathrm{i}}$ insensitive, but slower recovery of the fast component is promoted by increased $\left[\mathrm{Ca}^{2+}\right]_{\text {i. }}$ The $\left[\mathrm{Ca}^{2+}\right]_{\mathrm{i}}$ independence of rapid recovery of the slow component is opposed to what is expected from the release model proposed here, because increased basal $\left[\mathrm{Ca}^{2+}\right]_{\mathrm{i}}$ is expected to be necessary for a conversion from the refilled state $X$ into state $X^{\prime}$ leading to reduced release probabilities, as mentioned before. Probably, this can be solved by assuming refilling into state $X^{\prime}{ }_{0 \mathrm{Ca}}$ instead of $X_{0 \mathrm{Ca}}$. Then, the refilling rate itself would determine the recovery rate of the slow component, while the $\mathrm{Ca}^{2+}$ and Calmodulin sensitive recovery of the fast component (Sakaba and Neher, 2001b) might resemble the conversion rate from $X^{\prime}$ to $X$. However, a quantitative comparison of the finding by Wu and Borst (1999), and by Sakaba and Neher (2001b) with predictions by the model introduced here cannot be made yet, because the exact $\left[\mathrm{Ca}^{2+}\right]_{i}$ in between the two stimuli applied has not been determined.

\section{Physiological consequences of biphasic release}

It was suggested, that a single pool of readily releasable vesicles exists at the calyx of Held synapse, and that these vesicles all share the same mechanism underlying vesicle release. Furthermore, each vesicle can shift between two states, one of them with high (state $\mathrm{X}$, Fig. $24 \mathrm{E}$ ), and another with relatively low release probability (state $X^{\prime}$ ). This mechanism, termed the single pool model with intrinsic heterogeneity, leads to depression of peak EPSC amplitudes, before real depletion of the RRP sets in. Even in response to a single AP, the single pool model with intrinsic heterogeneity predicts about $28 \%$ of fast vesicles released less, as compared to the simple model without convertibility, while the contribution of fast to total release in response to a single AP was more than $97 \%$ (simulations not shown). Reduced amounts of fast release by conversion to a more 'reluctantly' releasing state $X$ ' might decrease the extent of multivesicular release (Meyer et al., 2001; Sun and Wu, 2001; Wadiche and Jahr, 2001; Wölfel and Schneggenburger, 2003), thus retarding saturation of synaptic transmission (Silver et al., 1996; Liu et al., 1999; Mainen et al., 1999; McAllister and Stevens, 2000; see also Hsu et al., 1996). Moreover, during high frequency stimulation of the 
calyx of Held (Spirou et al., 1990; Taschenberger and von Gerdorff, 2000), progressive RRP depletion can lead to synaptic short-term depression (for a review, see Schneggenburger et al., 2002). The model introduced in this study predicts a presynaptic mechanism of shortterm depression (see also, Hsu et al., 1996; Bellingham and Walmsley, 1999) in addition to other mechanisms, such as presynaptic pool depletion (Schneggenburger et al., 2002), or postsynaptic receptor desensitization (Trussel et al., 1993; Otis et a., 1996; Neher and Sakaba, 2001), and saturation (Neher and Sakaba, 2001; Sun and Wu, 2001). The conversion into a 'reluctantly' releasing state $X$ ' is expected to preserve vesicles for later stimulation, as observed in experiments (Figs. $16-17,18 C-D, 20 A 1,21-22$; Wu and Borst, 1999). In between periods of high frequency stimulation, phases of low-frequency stimulation can lead to recovery of the fast release component from depression by conversion from state $X^{\prime}$ to $X$. This conversion is possibly modulated by Calmodulin (Sakaba and Neher, 2001b), but differs from real refilling (Sakaba and Neher, 2001b). Thus, an intrinsic mechanism for presynaptic short-term depression, as proposed here, would allow for dynamic regulation of short-term plasticity in synaptic transmission, depending on intrinsic properties of the release mechanism, as well as on second messenger modulation of its 'resensitization' rate.

Given that synchronous release comes from the state $X$, while asynchronous release is determined by release from the 'desensitized' state $X$ ', 'resensitization' into state $X$ during a period of low frequency stimulation will increase the contribution of synchronous release, thereafter. Thus, the duration of a low frequency stimulation period will shape release at subsequent high-frequency firing, and the timing of sequences of high-, and low-frequency firing might contribute to information coding, additionally to AP frequency coding.

The molecular mechanism for a transition between two fusion-competent states with high and low release probabilities, respectively, remains to be studied. Synaptotagmin 1 is the most likely candidate for the $\mathrm{Ca}^{2+}$ sensor triggering fusion (Brose et al., 1992; Chapman and Jahn, 1994; Davis et al., 1999; Fernández-Chacón et al., 2001; Sugita et al., 2002; Sørensen et al., 2003; for reviews, see Koh and Bellen, 2003; Yoshihara et al., 2003). Given, that Synaptotagmin 1 triggers vesicle fusion by $\mathrm{Ca}^{2+}$ binding, stabilization of the fast release component is unlikely to be mediated by Synaptotagmin 1 (Sørensen et al., 2003), and therefore it should not determine the conversion between the proposed fast and slow releasing states $X$ and $X^{\prime}$. Voets et al. (2001) demonstrated that Synaptotagmin 1 is necessary for fast release of large dense core vesicles in chromaffin cells, while slow release does not seem to depend on Synaptotagmin 1 (see also Sørensen, 2004). This speaks 
against an identity of the $\mathrm{Ca}^{2+}$ sensors for fast and slow release in chromaffin cells, however, a direct comparison to central synapses such as the calyx of Held is difficult. Here, small synaptic vesicles account for release, and release rates are about tenfold faster as compared to chromaffin cells (compare Fig. 14, and Schneggenburger and Neher, 2000, to Voets, 2000). At the calyx of Held synapse, Synaptotagmin 2, not Synaptotagmin 1, is expressed (Wölfel and Schneggenburger, unpublished data), however, both show similar $\left[\mathrm{Ca}^{2+}\right]_{\mathrm{i}}$ dependences (Sugita et al., 2002). To test for a comparable role of Synaptotagmin 2 in the calyx of Held as compared to Synaptotagmin 1 in other systems, knock-out mice or regionspecific injection (Wimmer et al., 2004) of siRNA (Hannon and Rossi, 2004) could be used to block Synaptotagmin function. In context of the single pool model with intrinsic heterogeneity proposed here, Synaptotagmin 1 or 2 presumably underlies $\mathrm{Ca}^{2+}$ binding for both, the fast and the slow release component.

A molecular mechanism for conversion between the high and the low release probability state $X$ and $X^{\prime}$ might be based on differential SNARE complex formation (Voets et al., 1999; Sørensen, 2004), with a tight trans-SNARE complex promoting the fast releasing state $X$ (Chen et al., 1999; Scales et al., 2000; Voets, 2000; Sørensen, 2004). Point mutations in SNAP-25 to inhibit full priming at certain priming stages (Sørensen JB, personal communication) might have impact on the transition between a fast and a slowly releasing state $X$ and $X^{\prime}$, and therefore might clarify the molecular mechanism underlying 'desensitization' of release, as proposed here.

\section{Physiological significance of asynchronous release}

The biphasic behavior of synaptic release presumably accounts for the previously observed appearance of a synchronous, and an asynchronous component of release (Borges et al., 1995; Isaacson and Walmsley, 1995; Diamond and Jahr, 1995; Borst and Sakmann, 1996; Neher and Sakaba, 2001) during high frequency stimulation (Sakaba and Neher, 2001a; see also Fig. 10). The fast release component determines synchronous activity during single, or low frequency $\mathrm{AP}$ stimulation, and at high $\mathrm{AP}$ frequencies, the synchronous component depresses (Spirou et al., 1990; Taschenberger and von Gerdorff, 2000). Simultaneously, release from an asynchronous component accumulates, not only determined by residual glutamate in the synaptic cleft, but probably also due to inhomogeneous release probabilities within the RRP (Neher and Sakaba, 2001; Sakaba and Neher, 2001a; Fig. 14). Increased amounts of asynchronous release, or release from the slow component, might be important for short-term modulation of information transmission such as during high conductances states of neocortical neurons (for a review, see Destexhe et al., 2003). Here, increased 
membrane conductances have a shunting effect leading to decreased responsiveness of a neuron. Enhanced background noise, on the other hand, leads to a changed slope in the stimulus-response dependence. Both effects in combination induce a gain change in the responsiveness of a neuron (Hô and Destexhe, 2000; Chance et al., 2003). At the calyx of Held synapse, increased amounts of asynchronous release might have a similar effect, changing AP initiation probability. However, since the probability of postsynaptic AP generation is high in response to a single presynaptic AP, with a threshold as low as $0.5 \mathrm{nA}$ (von Gerdorff and Borst, 2002), and since the EPSC peak amplitude during high frequency stimulation depresses not more than to $1 \mathrm{nA}$ (Taschenberger and von Gersdorff, 2000), the impact of such a modulatory effect on postsynaptic AP initiation probability remains vague. 


\section{References}

Abenavoli A, Forti L, Bossi M, Bergamaschi A, Villa A, Malgaroli A (2002): Multimodal quantal release at individual hippocampal synapses: evidence for no lateral inhibition. J. Neurosci. 22(15): 6336 - 6346

Aumann Y, Parnas H (1991): Evaluation of the time course of neurotransmitter release from the measured PSC and MPSC. Bull. Math. Biol. 53: 537 - 555

Barbour B, Keller BU, Llano I, Marty A (1994): Prolonged presence of glutamate during excitatory synaptic transmission to cerebellar Purkinje cells. Neuron 12: $1331-1343$

Bellingham MC, Walmsley B (1999): A novel presynaptic inhibitory mechanism underlies paired pulse depression at a fast central synapse. Neuron 23(1): 159 - 170

Blank PS, Cho MS, Vogel SS, Kaplan D, Kang A, Malley J, Zimmerberg J. (1998a): Submaximal responses in calcium-triggered exocytosis are explained by differences in the calcium sensitivity of individual secretory vesicles. J. Gen. Physiol. 112(5): $559-67$

Blank PS, Vogel SS, Cho MS, Kaplan D, Bhuva D, Malley J, Zimmerberg J (1998b): The calcium sensitivity of individual secretory vesicles is invariant with the rate of calcium delivery. J. Gen. Physiol. 112(5): 569 - 576

Bollmann JH, Sakmann B, Borst JG (2000): Calcium sensitivity of glutamate release in a calyx-type terminal. Science 289: 953 - 957

Borges S, Gleason E, Turelli M, Wilson M (1995): The kinetics of quantal transmitter release from retinal amacrine cells. Proc. Natl. Acad. Sci. USA 92(15): 6896 - 6900

Borst JGG, Helmchen F, Sakmann B (1995): Pre- and postsynaptic whole-cell recordings in the medial nucleus of the trapezoid body of the rat. J. Physiol. 489(3): 825 - 840

Borst JGG, Sakmann B (1995): Calcium current during a single action potential in a large presynaptic terminal of the rat brainstem. J. Physiol. 506(1): 143 - 157

Borst JGG, Sakmann B (1996): Calcium influx and transmitter release in a fast CNS synapse. Nature 383: $431-434$

Borst JG, Sakmann B (1998): Calcium current during a single action potential in a large presynaptic terminal of the rat brainstem. J. Physiol. 506(1): $143-157$

Brose N, Petrenko AG, Südhof TC, Jahn R (1992): Synaptotagmin: a calcium sensor on the synaptic vesicle surface. Science 256(5059): 1021 - 1025

Chance FS, Abbott LF, Reyes AD (2002): Gain modulation from background synaptic input. Neuron 35(4): $773-782$

Chen YA, Scales SJ, Patel SM, Doung YC, Scheller RH (1999): SNARE complex formation is triggered by $\mathrm{Ca}^{2+}$ and drives membrane fusion. Cell 97(2): $165-174$

Clamann HP, Mathis J, Luscher HR (1989): Variance analysis of excitatory postsynaptic potentials in cat spinal motoneurons during posttetanic potentiation. J. Neurophysiol. 61(2): 403 - 416

Clements JD, Lester RAJ, Tong G, Jahr CE, Westbrook GL (1992): The time course of glutamate in the synaptic cleft. Science 258: 1498 - 1501

Cohen I, van der Kloot W, Attwell D (1981): The timing of channel opening during miniature end-plate currents. Brain Res. 223: 185 - 189 
Davis AF, Bai J, Fasshauer D, Wolowick MJ, Lewis JL, Chapman ER (1999): Kinetics of synaptotagmin responses to $\mathrm{Ca}^{2+}$ and assembly with the core SNARE complex onto membranes. Neuron 24(2):363 - 376

Denk W, Delaney KR, Gelperin A, Kleinfeld D, Strowbridge BW, Tank DW, Yuste R (1994): Anatomical and functional imaging of neurons using 2-photon laser scanning microscopy. J. Neurosci. Methods 54(2): 151 - 162, review

Destexhe A, Rudolph M, Pare D (2003): The high-conductance state of neocortical neurons in vivo. Nat. Rev. Neurosci. 4(9): 739 - 751, review

Diamond JS, Jahr CE (1995): Asynchronous release of synaptic vesicles determines the time course of the AMPA receptor-mediated EPSC. Neuron 15(5): 1097 - 1107

Dobrunz LE, Huang EP, Stevens CF (1997): Very short-term plasticity in hippocampal synapses. Proc. Natl. Acad. Sci. USA 94(26): 14843 - 14847

Edwards FA, Konnerth A, Sakmann B, Takahashi T (1989): A thin slice preparation for patch clamp recordings from neurons of the mammalian central nervous system. Pflüg. Arch. 414: $600-612$

Ellis-Davies GC (2003): Development and application of caged calcium. Methods Enzymol. 360:226 238

Ellis-Davies GC, Kaplan JH, Barsotti RJ (1996): Laser photolysis of caged calcium: rates of calcium release by nitrophenyl-EGTA and DM-nitrophen. Biophys. J. 70(2): 1006 - 1016

Felmy F, Neher E, Schneggenburger R (2003): Probing the intracellular calcium sensitivity of transmitter release during synaptic facilitation. Neuron 37(5): $801-811$

Fernández-Chacón R, Südhof TC (1999): Genetics of synaptic vesicle function: toward the complete functional anatomy of an organelle. Annu. Rev. Physiol. 61:753-76, review

Fernández-Chacón R, Königstorfer A, Gerber SH, Garcia J, Matos MF, Stevens CF, Brose N, Rizo J, Rosenmund C, Südhof TC (2001): Synaptotagmin I functions as a calcium regulator of release probability. Nature 410(6824): $41-49$

Forsythe ID (1994): Direct patch recording from identified presynaptic terminals mediating glutamatergic EPSCs in the rat CNS, in vitro. J. Physiol. 479(3): 381 - 387

Forsythe ID, Barnes-Davies M, Brew HM (1995): The calyx of Held: a model for transmission at mammalian glutamatergic synapses. In: Excitatory amino acids and synaptic transmission, $2^{\text {nd }}$ edition. Wheal HV, editor. Academic Press 133 - 144

Geppert M, Archer BT 3rd, Südhof TC (1991): Synaptotagmin II. A novel differentially distributed form of synaptotagmin. J. Biol. Chem. 266(21): 13548 - 13552

Gillis KD (1995): Techniques for membrane capacitance measurements. In: Single-channel recording, 2nd edition. Sakmann B and Neher E, editors. Plenum Press, New York pp. $155-198$

Goda Y, Stevens CF (1994): Two components of transmitter release at a central synapse. Proc. Natl. Acad. Sci. USA 91(26): 12942 - 12946

Grynkievicz G, Poenie M, Tsien R (1985): A new generation of $\mathrm{Ca}^{2+}$ indicators with greatly improved fluorescence properties. J. Biol. Chem. 260: 3440 - 3450

Guerini $\mathrm{D}$ (1998): The $\mathrm{Ca}^{2+}$ pumps and the $\mathrm{Na}^{+} / \mathrm{Ca}^{2+}$ exchangers. BioMetals 11: $319-330$

Hamill OP, Marty E, Neher E, Sakmann B, Sigworth FJ (1981): Improved patch clamp techniques for high-resolution current recordings from cells and cell-free patches. Pflüg. Arch. 391: 85-100 
Hannon GJ, Rossi JJ (2004): Unlocking the potential of the human genome with RNA interference. Nature 431(7006): 371 - 378, review

Harafuji H, Ogawa $Y(1980)$ : Re-examination of the apparent binding constant of ethylene glycol bis(beta-aminoethyl ether)-N,N,N',N'-tetraacetic acid with calcium around neutral $\mathrm{pH}$. J. Biochem. (Tokyo) 87(5): $1305-1312$

Heidelberger R, Heinemann C, Neher E, Matthews G (1994): Calcium dependence of the rate of exocytosis in a synaptic terminal. Nature 371: $513-515$

Heinemann C, Chow RH, Neher E, Zucker RS (1994): Kinetics of the secretory response in bovine chromaffin cells following flash photolysis of caged $\mathrm{Ca}^{2+}$. Biophys. J. 67: $2546-2557$

Held H (1893): Die centrale Gehörleitung. Arch. Anat. u. Physiol. 201 - 248

Helmchen F, Borst JGG, Sakmann B (1997): Calcium dynamics associated with a single action potential in a CNS presynaptic Terminal. Biophysical J. 72: $1458-1471$

Hô N, Destexhe A (2000): Synaptic background activity enhances the responsiveness of neocortical pyramidal neurons. J. Neurophysiol. 84(3): 1488 - 1496

Horrigan FT, Bookman RJ (1994): Releasable pools and the kinetics of exocytosis in adrenal chromaffin cells. Neuron 13(5): $1119-11129$

Hsu SF, Augustine GJ, Jackson MB (1996): Adaptation of $\mathrm{Ca}^{2+}$-triggered exocytosis in presynaptic terminals. Neuron 17(3): $501-512$

Hsu SF, Jackson MB (1996): Rapid exocytosis and endocytosis in nerve terminals of the rat posterior pituitary. J. Physiol. 494(2): 539 - 553

Hyrc KL, Bownik JM, Goldberg MP (2000): Ionic selectivity of low-affinity ratiometric calcium indicators: mag-Fura-2, Fura-2FF and BTC. Cell Calcium 27(2): $75-86$

Isaacson JS, Walmsley B (1995): Counting quanta: direct measurements of transmitter release at a central synapse. Neuron 15(4): 875 - 884

Jahn R, Lang T, Südhof TC (2003): Membrane fusion. Cell 112(4): 519 - 533, review

Jonas P, Major G, Sakmann B (1993): Quantal components of unitary EPSCs at the mossy fiber synapse on CA3 pyramidal cells of rat hippocampus. J. Physiol. (London) 472: 615 - 663

Jones AW, Smith DA, Watkins JC (1984): Structure-activity relations of dipeptide antagonists of excitatory amino acids. Neuroscience 13(2): $573-581$

Kandel ER, Schwartz JH, Jessell TM (2000): Principles of neural science. McGraw-Hill, 4th edit.

Kaplan JH, Ellis-Davies GCR (1988): Photolabile chelators for the rapid photorelease of divalent cations. Proc. Natl. Acad. Sci. USA 85: $6571-6575$

Katz B. (1969): The release of neurotransmitter substances. Liverpool: Liverpool University Press

Katz LC, Dalva MB (1994): Scanning laser photostimulation: a new approach for analyzing brain circuits. J. Neurosci. Methods. 54(2): 205 - 218, review

Katz B, Miledi R (1965): The effect of temperature on the synaptic delay at the neuromuscular junction. J. Physiol. 181(3): $656-670$

Koh TW, Bellen HJ (2003): Synaptotagmin I, a $\mathrm{Ca}^{2+}$ sensor for neurotransmitter release. Trends Neurosci. 26(8): 413 - 422, review 
Liu G, Choi S, Tsien RW (1999): Variability of neurotransmitter concentration and nonsaturation of postsynaptic AMPA receptors at synapses in hippocampal cultures and slices. Neuron 22(2): 395 409

Llinas R, Steinberg IZ, Walton K (1981): Relationship between presynaptic calcium current and postsynaptic potential in squid giant synapse. Biophys. J. 33(3): $323-351$

Lou XL, Scheuss V, Schneggenburger R (2005): Allosteric modulation of the presynaptic $\mathrm{Ca}^{2+}$ sensor for vesicle fusion by phorbol esters. submitted

Mainen ZF, Malinow R, Svoboda K (1999) Synaptic calcium transients in single spines indicate that NMDA receptors are not saturated. Nature 399(6732):151 - 155

Marciani MG, Louvel J, Heinemann U (1982): Aspartate-induced changes in extracellular free calcium in 'in vitro' hippocampal slices of rats. Brain Res. 238(1): 272 - 277

Martell AE, Smith R (1974): Critical stability constants, vol. 1. Plenum Press, New York

Marty A, Neher E (1995): Tight-seal whole-cell recording. In: Single-channel recording, $2^{\text {nd }}$ edition. Sakmann B and Neher E, Editors. Plenum Press, New York. 31 - 52

McAllister AK, Stevens CF (2000): Nonsaturation of AMPA and NMDA receptors at hippocampal synapses. Proc. Natl. Acad. Sci. USA 97(11): 6173 - 6178

Mennerick S, Zorumski CF (1995): Presynaptic influence on the time course of fast excitatory synaptic currents in cultured hippocampal cells. J. Neurosci. 15: $3178-3192$

Mennerick S, Matthews G (1996): Ultrafast exocytosis elicited by calcium current in synaptic terminals of retinal bipolar neurons. Neuron 17(6): 1241 - 1249

Meyer A (1999): Untersuchungen zur Wahrscheinlichkeit der Transmitterfreisetzung an einer zentralen Synapse. Diploma thesis, Max-Planck-Institute for biophysical Chemistry Göttingen Germany, 88 - 89

Meyer AC, Neher E, Schneggenburger R (2001): Estimation of quantal size and number of functional active zones at the calyx of Held synapse by nonstationary EPSC variance analysis. J. Neurosci. 21(20): 7889 - 7900

McCray JA, Fidler-Lim N, Ellis-Davies GCR, Kaplan JH (1992): Rate of release of $\mathrm{Ca}^{2+}$ following laser photolysis of the DM-nitrophen $\mathrm{Ca}^{2+}$ complex. Biochemistry 31: $8856-8861$

Monod J, Wyman J, Changeux JP (1965): On the nature of allosteric transitions: a plausible model. J. Mol. Biol. 12:88 - 118

Moser T, Neher E (1997): Rapid exocytosis in single chromaffin cells recorded from mouse adrenal slices. J. Neurosci. 17(7): $2314-2323$

Murthy VN, Sejnowski TJ, Stevens CF (1997): Heterogeneous release properties of visualized individual hippocampal synapses. Neuron 18(4): 599 - 612

Naraghi M (1997): T-jump study of calcium binding kinetics of calcium chelators. Cell Calcium 22: 255 $-268$

Neher E (1998): Vesicle pools and $\mathrm{Ca}^{2+}$ microdomains: new tools for understanding their roles in neurotransmitter release. Neuron 20(3): $389-399$, review

Neher E, Sakaba T (2001): Combining deconvolution and noise analysis for the estimation of transmitter release rates at the calyx of Held. J. Neurosci. 21(2): 444 - 461 
Neher E, Sakmann B (1976): Single-channel currents recorded from membrane of denervated frog muscle fibers. Nature 260: $799-802$

Neher E, Zucker RS (1993): Multiple Calcium-dependent processes related to Secretion in bovine chromaffin cells. Neuron 10: $21-30$

Oertel D (1997): Encoding of timing in the brain stem auditory nuclei of vertebrates. Neuron 19(5): 959 - 962, review

Oleskevich S, Clements J, Walmsley B. (2000): Release probability modulates short-term plasticity at a rat giant terminal. J. Physiol. 524(2): 513 - 523

Otis T, Wu YC, Trussell LO (1996): Direct measurement of AMPA receptor desensitization induced by glutamatergic synaptic transmission. J. Neurosci. 16: 7496 - 7504

Paxinos G, Watson C (1986): The rat brain atlas. Academic Press Inc. USA, $2^{\text {nd }}$ edit. Fig. 79

Philipson KD, Nicoll DA (2000): Sodium-Calcium exchange: a molecular perspective. Annu. Rev. Physiol. 62: $111-133$

Pusch M, Neher E (1988): Rates of diffusional exchange between small cells and a measuring patch pipette. Pflüg. Arch. 411: 204 - 211

Rahamimoff R, Yaari Y (1973): Delayed release of transmitter at the frog neuromuscular junction. $J$. Physiol. 228(1): $241-257$

Reid CA, Clements JD (1999): Postsynaptic expression of long-term potentiation in the rat dentate gyrus demonstrated by variance-mean analysis. J. Physiol. 518(1): 121 - 130

Sakaba T, Neher E (2001a): Quantitative relationship between transmitter release and Calcium current at the calyx of Held synapse. J. Neurosci. 21(2): 462 - 476

Sakaba T, Neher E (2001b): Calmodulin mediates rapid recruitment of fast-releasing synaptic vesicles at a calyx-type synapse. Neuron 32: $1119-1131$

Sakaba T, Tachibana M, Matsui K, Minami N (1997): Two components of transmitter release in retinal bipolar cells: exocytosis and mobilization of synaptic vesicles. Neurosci. Res. 27(4): 357 - 370

Sätzler K, Söhl LF, Bollmann JH, Borst JG, Frotscher M, Sakmann B, Lübke JH (2002): Threedimensional reconstruction of a calyx of Held and its postsynaptic principal neuron in the medial nucleus of the trapezoid body. J. Neurosci. 22(24): 10567 - 10579

Scales SJ, Chen YA, Yoo BY, Patel SM, Doung YC, Scheller RH (2000): SNAREs contribute to the specificity of membrane fusion. Neuron 26(2): 457 - 464

Scheuss V, Neher E (2001): Estimating synaptic parameters from mean, variance, and covariance in trains of synaptic responses. Biophys. J. 81(4): $1970-1989$

Schneggenburger R, Meyer AC, Neher E (1999): Released fraction and total size of a pool of immediately available transmitter quanta at a calyx synapse. Neuron 23: 399 - 409

Schneggenburger R, Neher E (2000): Intracellular calcium dependence of transmitter release rates at a fast central synapse. Nature 406: $889-893$

Schneggenburger R, Sakaba T, Neher E (2002): Vesicle pools and short-term synaptic depression: lessons from a large synapse. Trends. Neurosci. 25(4): 206 - 212, review

Schneggenburger R, Zhou Z, Konnerth A, Neher E (1993): Fractional contribution of calcium to the cation current through glutamate receptor channels. Neuron 11(1): 133 - 143 
Seward EP, Nowycky MC (1996): Kinetics of stimulus-coupled secretion in dialyzed bovine chromaffin cells in response to trains of depolarizing pulses. J. Neurosci. 16(2): $553-562$

Silver RA, Cull-Candy SG, Takahashi T (1996): Non-NMDA glutamate receptor occupancy and open probability at a rat cerebellar synapse with single and multiple release sites. J. Physiol. 494(1): 231 250

Silver RA, Momiyama A, Cull-Candy SG (1998): Locus of frequency-dependent depression identified with multiple-probability fluctuation analysis at rat climbing fibre-Purkinje cell synapses. J. Physiol. 510(3): 881 - 902

Spirou WA, Brownell WE, Zidanic M (1900): Recordings from cat trapezoid body and HRP labeling of globular bushy cell axons. J. Neurophysiol. 63: $1169-1190$

Sørensen JB (2004): Formation, stabilisation and fusion of the readily releasable pool of secretory vesicles. Pflügers Arch. 448(4): 347 - 362

Sørensen JB, Fernández-Chacón R, Südhof TC, Neher E (2003): Examining synaptotagmin 1 function in dense core vesicle exocytosis under direct control of $\mathrm{Ca}^{2+}$. J. Gen. Physiol. 122(3): $265-276$

Stevens CF, Wang Y (1995): Facilitation and depression at single central synapses. Neuron 14(4): $795-802$

Sugita S, Shin OH, Han W, Lao Y, Südhof TC (2002): Synaptotagmins form a hierarchy of exocytotic $\mathrm{Ca}^{2+}$ sensors with distinct $\mathrm{Ca}^{2+}$ affinities. EMBO J. 21(3): $270-280$

Sun JY, Wu LG (2001): Fast kinetics of endocytosis revealed by simultaneous measurements of presynaptic capacitance and postsynaptic currents at a central synapse. Neuron 30: $171-182$

Sun JY, Wu XS, Wu LG (2002): Single and multiple vesicle fusion induce different rates of endocytosis at a central synapse. Nature 417: $555-559$

Taschenberger H, Leão RM, Rowland KC, Spirou GA, von Gersdorff H (2002): Optimizing synaptic architecture and efficiency for high-frequency transmission. Neuron 36(6): 1127 - 1143

Taschenberger H, von Gersdorff H (2000): Fine-tuning an auditory synapse for speed and fidelity: developmental changes in presynaptic waveform, EPSC kinetics, and synaptic plasticity. J. Neurosci. 20(24): $9162-9173$

Triller A, Korn H (1985): Activity-dependent deformations of presynaptic grids at central synapses. J. Neurocytol. 14(2): $177-192$

Trussell LO (1997): Cellular mechanisms for preservation of timing in central auditory pathways. Curr. Opin. Neurobiol. 7(4): 487 - 492, review

Trussell LO, Zhang S, Raman IM (1993): Desensitization of AMPA receptors upon multiquantal neurotransmitter release. Neuron. 10: 1185 - 96

van der Kloot W (1988): Estimating the timing of quantal release during end-plate currents at the frog neuromuscular junction. J. Physiol. (London) 402: 595 - 603

Voets $\mathrm{T}$ (2000): Dissection of three $\mathrm{Ca}^{2+}$-dependent steps leading to secretion in chromaffin cells from mouse adrenal slices. Neuron 28(2): 537 - 545

Voets T, Moser T, Lund PE, Chow RH, Geppert M, Südhof TC, Neher E (2001): Intracellular calcium dependence of large dense-core vesicle exocytosis in the absence of synaptotagmin I. Proc. Natl. Acad. Sci. USA 98(20):11680 - 11685 
Voets T, Neher E, Moser T (1999): Mechanisms underlying phasic and sustained secretion in chromaffin cells from mouse adrenal slices. Neuron 23(3): 607 - 615

von Gersdorff H, Borst JG (2002): Short-term plasticity at the calyx of held. Nat. Rev. Neurosci. 3(1): $53-64$, review

von Gersdorff H, Sakaba T, Berglund K, Tachibana M (1998): Submillisecond kinetics of glutamate release from a sensory synapse. Neuron 21(5): $1177-1188$

Wadiche JI, Jahr CE (2001): Multivesicular release at climbing fiber-Purkinje cell synapses. Neuron 32(2): $301-313$

Walmsley B, Edwards FR, Tracey DJ (1988): Nonuniform release probabilities underlie quantal synaptic transmission at a mammalian excitatory central synapse. J. Neurophysiol. 60(3): 889 - 908

Watkins JC, Evans RH (1981): Excitatory amino acid transmitters. Annu. Rev. Pharmacol. Toxicol. 21: $165-204$

Wimmer VC, Nevian T, Kuner T (2004): Targeted in vivo expression of proteins in the calyx of Held. Pflügers Arch. Sep 28

Wölfel M, Schneggenburger R (2003): Presynaptic capacitance measurements and $\mathrm{Ca}^{2+}$ uncaging reveal submillisecond exocytosis kinetics and characterize the $\mathrm{Ca}^{2+}$ sensitivity of vesicle pool depletion at a fast CNS synapse. J. Neurosci. 23(18): 7059 - 7068

Wu LG, Borst JGG (1999): The reduced release probability of releasable vesicles during recovery from short-term synaptic depression. Neuron 23: $821-832$

Yawo H (1999): Two components of transmitter release from the chick ciliary presynaptic terminal and their regulation by protein kinase C. J. Physiol. 516 (2): 461 - 470

Yoshihara M, Adolfsen B, Littleton JT (2003): Is synaptotagmin the calcium sensor? Curr. Opin. Neurobiol. 3(3): $315-323$, review

Xu T, Binz T, Niemann H, Neher E (1998): Multiple kinetic components of exocytosis distinguished by neurotoxin sensitivity. Nat. Neurosci. 1(3): 192 - 200

Xu T, Rammner B, Margittai M, Artalejo AR, Neher E, Jahn R (1999): Inhibition of SNARE complex assembly differentially affects kinetic components of exocytosis. Cell 99(7): $713-722$

Zigmond MJ, Bloom FE, Landis SC, Roberts JL, Squire LR (1999): Fundamental neuroscience. Academic Press, 1st edit.

Zucker RS (1992): Effect of photolabile calcium chelators on fluorescent calcium indicators. Cell Calcium 13: $29-40$

Zucker RS (1993): The calcium concentration clamp: spikes and reversible pulses using the photolabile chelator DM-nitrophen. Cell Calcium 14(2): 87 - 100 


\section{Abbreviations}

\begin{tabular}{|c|c|}
\hline AMPA & alpha-amino-3-hydroxy-5-methyl-4-isoxazolepropionic acid \\
\hline AP & action potential \\
\hline $\mathrm{Ag} / \mathrm{AgCl}$ & silver/silver-chloride \\
\hline ATP & adenosine-triphosphate \\
\hline $\mathrm{b}$ & cooperativity factor in $\mathrm{Ca}^{2+}$ binding model \\
\hline$\left[\mathrm{Ca}^{2+}\right]_{\mathrm{i}}$ & intracellular Calcium concentration \\
\hline$\left[\mathrm{Ca}^{2+}\right]_{0}$ & extracellular Calcium concentration \\
\hline $\mathrm{C}_{\mathrm{m}}$ & membrane capacitance \\
\hline CNS & central nervous system \\
\hline CTZ & cyclothiazide \\
\hline D-AP5 & D-(-)-2-amino-5-phosphonopentanoic acid \\
\hline DMN & DM-nitrophen \\
\hline DPTA & 1,3-Diamino-2-propanol-N,N,N',N'-tetraacetic acid \\
\hline EGTA & Ethylene glycol-bis(2-aminoethylether)-N,N,N',N'-tetraacetic acid \\
\hline EM & electron microscopy \\
\hline EPSC & excitatory postsynaptic current \\
\hline FRP & fast releasable pool \\
\hline$\gamma$ & fusion rate in $\mathrm{Ca}^{2+}$ binding model \\
\hline$\gamma$-DGG & $\gamma$-D-glutamylglycine \\
\hline GTP & guanosin-triphosphate \\
\hline HEPES & $\mathrm{N}$-(2-Hydroxyethyl)piperazine-N'-(2-ethanesulfonic acid) \\
\hline ISI & inter-stimulus interval \\
\hline $\mathrm{K}_{\mathrm{D}}$ & coefficient of dissociation \\
\hline $\mathrm{K}_{\mathrm{eff}}$ & efficient coefficient of dissociation \\
\hline$k_{\text {off }}$ & off-rate in $\mathrm{Ca}^{2+}$ binding model \\
\hline $\mathrm{k}_{\mathrm{on}}$ & on-rate in $\mathrm{Ca}^{2+}$ binding model \\
\hline LSO & lateral superior olive \\
\hline mEPSC & miniature excitatory postsynaptic current \\
\hline MNTB & medial nucleus of the trapezoid body \\
\hline mPSC & miniature postsynaptic current \\
\hline NMDA & $\mathrm{N}$-methyl-D-aspartatic acid \\
\hline PSC & postsynaptic current \\
\hline$r_{\text {ref }}$ & refilling rate \\
\hline$r_{\text {ref, } \max }$ & maximal refilling rate \\
\hline Res & reserve pool \\
\hline RRP & readily-releasable pool \\
\hline $\mathrm{R}_{\mathrm{s}}$ & series resistance \\
\hline s.d. & standard deviation \\
\hline SRP & slowly releasable pool \\
\hline TEA & tetraethylammonium chloride \\
\hline TTX & tetrodotoxin \\
\hline VCN & ventral cochlear nucleus \\
\hline ves & vesicles \\
\hline $\mathrm{X}_{\mathrm{nCa}}$ & state of $\mathrm{Ca}^{2+}$ binding model with $\mathrm{n} \mathrm{Ca}^{2+}$ ions bound \\
\hline
\end{tabular}





\section{Acknowledgements}

Finally, I would like to sincerely thank everyone, who kindly supported me during the last years, while I was working on this thesis. On the job or in private life, they all have been a great support in many respects.

Sincere thanks to Prof. Dr. Erwin Neher, for giving me the chance to work in such a brilliant laboratory with an extraordinarily nice atmosphere, and for patient, fruitful, and very instructive discussions throughout the project.

Many thanks also to Prof. Dr. Andreas Stumpner, who gave me friendly support, being always available when I asked for help.

Very special thanks to Dr. PD Ralf Schneggenburger, who supervised me during the whole project. He was very patient and helpful at any time, and his integrity, openness, and friendly nature made my work in the past years a real pleasure.

Many thanks also to Irmgard Barteczko for helping me to overcome bureaucratic hurdles easily, and moreover, for creating such a friendly and pleasant atmosphere in the whole department.

The whole group of Synaptic Dynamics and Modulation around Ralf Schneggenburger, and also Dr. Takeshi Sakaba, were a fantastic help not only in respect to lab work and precious discussions, but also relating to lab life. Felix Felmy, Martin Müller, Natalia Korogod, Ralf Schneggenburger, Sigrid Schmidt, Takeshi Sakaba, Volker Scheuss, and Xue-Lin Lou were part of a wonderful group, always being around, when practical or theoretical help was needed. Thank you for your support, and for all the fun, I had!

Moreover, I would like to thank Veronica Müller for her kind organizational help when finishing this thesis, Frank Köhne, Frank Würriehausen, Michael Pilot and the crew of the animal house for their friendly technical assistance, and Edward Lemke for discussions and experimental help concerning spatial flash-homogeneity.

Enormous thanks to all my friends, who made my life as great as can be. During the last years, when I was busy with this thesis, especially Assiba Akoho and Fokko Steinbeisser, Biona Balzer, Birga Ipsen, Dirk Mederer and Anna Verena Weinand, Jeanette Wagener, Marco Luthe, Marco Melas and Petra Dierkes, Nils Wasmuth, Petra and Manuela Kiendler, Philip Schatz, and Torsten Curdt supported me with their wonderful friendship.

My biggest gratitude goes to my dear parents, Ingrid and Helmuth Wölfel, and my dear sister Katharina Wölfel. Because of their loving care, I was happy throughout my life, and moreover, could manage my scientific career, at least, up to this point. Thank you so much! 



\section{Curriculum vitae}

On October the $26^{\text {th }}$ in 1976, I, Markus Wölfel, was born as the son of my mother Ingrid Wölfel and my father Dr. Helmuth Wölfel in the German city Göttingen, having an Austrian nationality. I went to school from 1983 to 1996, and graduated with "Abitur" at the TheodorHeuß-Gymnasium in Göttingen, taking exams in the subjects biology, arts, mathematics and geography.

In the same year, I started as a student at the biological faculty of the Georg-AugustUniversity Göttingen. Passing exams in zoology, microbiology, physics and chemistry, I received the "Vordiplom" in 1998, and in 2001, I graduated as a Diploma Biologist, being examined in zoology, botany, physics, and computer science. My diploma thesis with the title "Calcium-gesteuerte Vesikelfusion in einer schnellen Synapse des Zentralnervensystems von Ratten” [engl.: "Calcium-regulated fusion of vesicles in a fast synapse of the central nervous system in rats"] was worked out in the group of Synaptic Dynamics and Modulation under supervision of the group leader Dr. PD Ralf Schneggenburger. The group is part of the department of Membrane Biophysics directed by Prof. Dr. Erwin Neher at the Max-PlanckInstitute of biophysical Chemistry in Göttingen, and Prof. Dr. Erwin Neher was also the coreferee of the Diploma thesis, whereas Prof. Dr. Andreas Stumpner of the Institute of Zoology of the Georg-August-University Göttingen was the main referee.

Immediately thereafter, in 2001 , I started this PhD thesis in the same group guided by Dr. PD Ralf Schneggenburger.

Meanwhile, I could contribute to the following publications:

- $\quad$ Wölfel M, Schneggenburger R (2003)

"Presynaptic capacitance measurements and $\mathrm{Ca}^{2+}$ uncaging reveal submillisecond exocytosis kinetics and characterize the $\mathrm{Ca}^{2+}$ sensitivity of vesicle pool depletion at a fast CNS synapse"

J. Neurosci. 23(18): 7059 - 7068

Fernández-Chacón R, Wölfel M, Nishimune H, Tabares L, Schmitz F, CastellanoMuñoz M, Rosenmund C, Montesinos ML, Sanes JR, Schneggenburger R, Südhof TC (2004)

"CSP $\alpha$ Prevents Presynaptic Degeneration"

Neuron 42(2) : 237 - 251 